A UNITED STATES

DEPARTMENT OF

COMMERCE

PUBLICATION

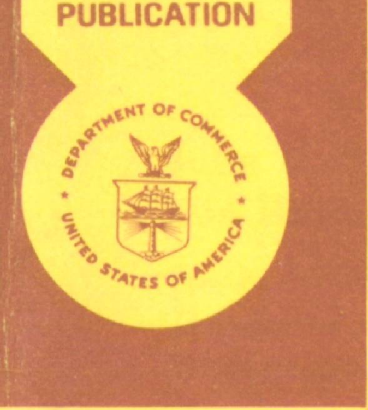

\title{
Platinum Resistance Thermometry
}

U.S. DEPARTMENT OF COMMERCE National

Bureau of Standards

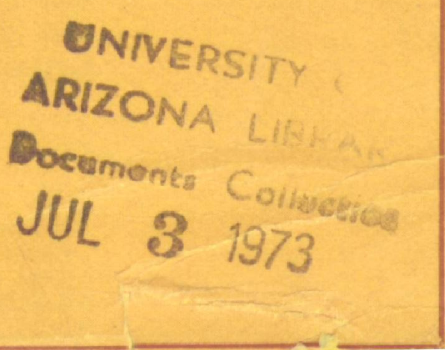




\section{NATIONAL BUREAU OF STANDARDS}

The National Bureau of Standards ${ }^{1}$ was established by an act of Congress March 3, 1901. The Bureau's overall goal is to strengthen and advance the Nation's science and technology and facilitate their effective application for public benefit. To this end, the Bureau conducts research and provides: (1) a basis for the Nation's physical measurement system, (2) scientific and technological services for industry and government, (3) a technical basis for equity in trade, and (4) technical services to promote public safety. The Bureau consists of the Institute for Basic Standards, the Institute for Materials Research, the Institute for Applied Technology, the Center for Computer Sciences and Technology, and the Office for Information Programs.

THE INSTITUTE FOR BASIC STANDARDS provides the central basis within the United States of a complete and consistent system of physical measurement; coordinates that system with measurement systems of other nations; and furnishes essential services leading to accurate and uniform physical measurements throughout the Nation's scientific community, industry, and commerce. The Institute consists of a Center for Radiation Research, an Office of Measurement Services and the following divisions:

Applied Mathematics - Electricity - Mechanics - Heat - Optical Physics Linac Radiation " - Nuclear Radiation" - Applied Radiation " - Quantum Electronics: - Electromagnetics $:$ - Time and Frequency ${ }^{3}$ - Laboratory Astrophysics : - Cryogenics ${ }^{3}$.

THE INSTITUTE FOR MATERIALS RESEARCH conducts materials research leading to improved methods of measurement, standards, and data on the properties of well-characterized materials needed by industry, commerce, educational institutions, and Government; provides advisory and research services to other Government agencies; and develops, produces, and distributes standard reference materials. The Institute consists of the Office of Standard Reference Materials and the following divisions:

Analytical Chemistry-Polymers-Metallurgy-Inorganic Materials-Reactor Radiation-Physical Chemistry.

THE INSTITUTE FOR APPLIED TECHNOLOGY provides technical services to promote the use of available technology and to facilitate technological innovation in industry and Government; cooperates with public and private organizations leading to the development of technological standards (including mandatory safety standards), codes and methods of test; and provides technical advice and services to Government agencies upon request. The Institute also monitors NBS engineering standards activities and provides liaison between NBS and national and international engineering standards bodies. The Institute consists of a Center for Building Technology and the following divisions and offices:

Engineering and Product Standards-Weights and Measures-Invention and Innovation-Product Evaluation Technology-Electronic Technology-Technical Analysis-Measurement Engineering-Building Standards and Code Services ${ }^{4}$-Housing Technology ${ }^{4}$-Federal Building Technology 4 -Structures, Materials and Life Safety ${ }^{4}$-Building Environment ${ }^{4}$-Technical Evaluation and Application ${ }^{4}$-Fire Technology.

THE INSTITUTE FOR COMPUTER SCIENCES AND TECHNOLOGY conducts research and provides technical services designed to aid Government agencies in improving cost effectiveness in the conduct of their programs through the selection, acquisition, and effective utilization of automatic data processing equipment; and serves as the principal focus within the executive branch for the development of Federal standards for automatic data processing equipment, techniques, and computer languages. The Center consists of the following offices and divisions:

Information Processing Standards-Computer Information-Computer Services -Systems Development-Information Processing Technology.

THE OFFICE FOR INFORMATION PROGRAMS promotes optimum dissemination and accessibility of scientific information generated within NBS and other agencies of the Federal Government; promotes the development of the National Standard Reference Data System and a system of information analysis centers dealing with the broader aspects of the National Measurement System; provides appropriate services to ensure that the NBS staff has optimum accessibility to the scientific information of the world, and directs the public information activities of the Bureau. The Office consists of the following organizational units:

Office of Standard Reference Data-Office of Technical Information and Publications-Library-Office of International Relations.

\footnotetext{
1 Headquarters and Laboratories at Gaithersburg, Maryland, unless otherwise noted; malling address Washington, D.C. 20234.

- Part of the Center for Radiation Research.

s Located at Boulder, Colorado 80302 .

- Part of the Center for Bullding Technology.
} 


\section{Platinum Resistance Thermometry}

John L. Riddle, George T. Furukawa, and Harmon H. Plumb

Institute for Basic Standards

National Bureau of Standards

Washington, D.C. 20234

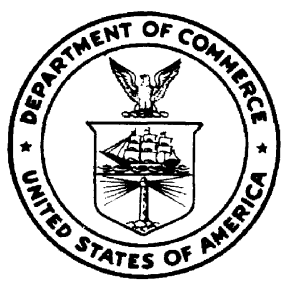

U.S. DEPARTMENT OF COMMERCE, Frederick B. Dent, Secretary NATIONAL BUREAU OF STANDARDS, Richard W. Roberts, Director

Issued April 1973 


\title{
Library of Congress Catalog Card Number: 72-60003
}

\author{
National Bureau of Standards Monograph 126 \\ Nat. Bur. Stand. (U.S.), Monogr. 126, 129 pages (Apr. 1972) \\ CODEN: NBSMA6
}

For sale by the Superintendent of Documents, U.S. Government Printing Office, Washington, D.C. 20402 Price: \$2.10, domestic postpaid: \$1.75, GPO Bookstore. Stock No. 0303-01052

(Order by SD Catalog No. C13.44:126). 


\section{Contents}

1. Introduction.

2. Background and basic concepts

2.1. Thermodynamic temperature scale.

2.2. Practical temperature scales...

3. Platinum resistance thermometer construction

4. Using the thermometer...

4.1. Mechanical treatment of SPRT.

4.2. Thermal treatment of SPRT.

4.3. Thermometer immersion...

4.4. Heating effects in SPRT

5. Resistance measurements.

5.1. Mueller bridge

5.1.1. Bridge ratio arms

5.1.2. Bridge current reversal.

5.2. A-C bridge

5.3. Potentiometric methods.

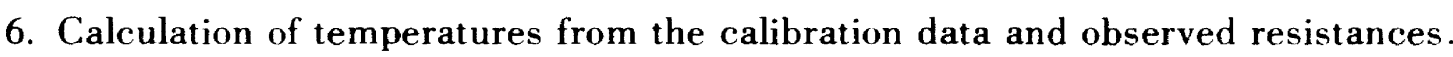

6.1. Temperatures from 0 to $630.74{ }^{\circ} \mathrm{C}$

6.2. Temperatures below $0{ }^{\circ} \mathrm{C}$

6.2.1. $-182.962{ }^{\circ} \mathrm{C}(90.188 \mathrm{~K})$ to $0{ }^{\circ} \mathrm{C}(273.15 \mathrm{~K})$

6.2.2. 13.81 to $90.188 \mathrm{~K}$.

6.2.2.1. 54.361 to $90.188 \mathrm{~K}$

6.2.2.2. 20.28 to $54.361 \mathrm{~K}$

6.2.2.3. 13.81 to $20.28 \mathrm{~K}$

6.2.3. Calibration tables between 13.81 and $273.15 \mathrm{~K}\left(0^{\circ} \mathrm{C}\right)$

6.3. Errors of temperature determinations

7. Calibration

7.1. Triple point of water.

7.2. Metal freezing points....

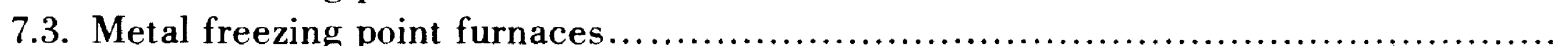

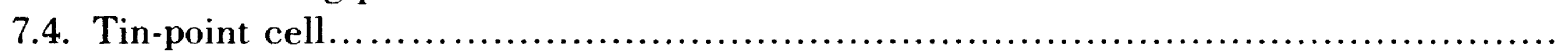

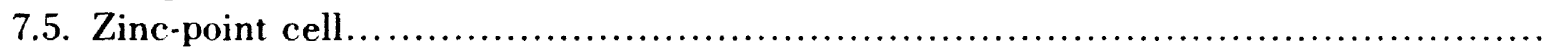

7.6. Oxygen normal boiling point.

7.7. Comparison calibration between 13.81 and $90.188 \mathrm{~K}$

8. References

9. Appendixes:

A. The International Practical Temperature Scale of 1968 (adopted by the Comite International des Poids et Measures).

B. Comparison of assigned fixed point values and interpolating formulae in the temperature ranges defined by the platinum resistance thermometer for ITS-27, ITS-48, IPTS-48, and IPTS-68. 
C. Tables of difference in the values of temperature between the IPTS-48 and IPTS-68 and between the NBS-1955 and IPTS-68 in the temperature range $13 \mathrm{~K}$ to $630^{\circ} \mathrm{C} \ldots$

D. Table of the function

$$
M\left(t^{\prime}\right)=0.045\left(\frac{t^{\prime}}{100{ }^{\circ} \mathrm{C}}\right)\left(\frac{t^{\prime}}{100{ }^{\circ} \mathrm{C}}-1\right)\left(\frac{t^{\prime}}{419.58{ }^{\circ} \mathrm{C}}-1\right)\left(\frac{t^{\prime}}{630.74{ }^{\circ} \mathrm{C}}-1\right){ }^{\circ} \mathrm{C}
$$

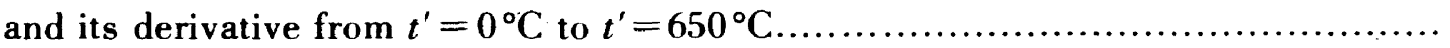

E. Tables of values of the reference function and its derivative from $T=13 \mathrm{~K}$ to $T=273.15 \mathrm{~K}$ and over the equivalent range of temperature with the argument in degrees Celsius...

F. Analysis of the first derivatives at $0^{\circ} \mathrm{C}$ of IPTS -68 platinum resistance thermometer

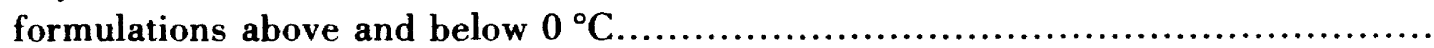

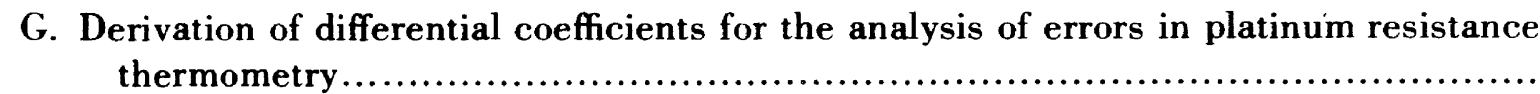

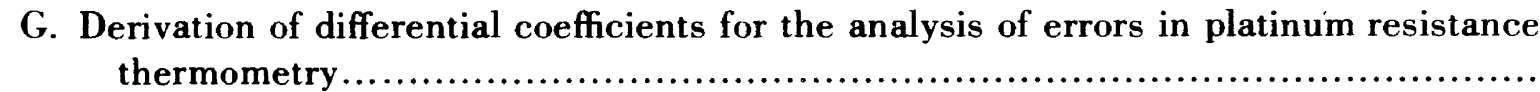

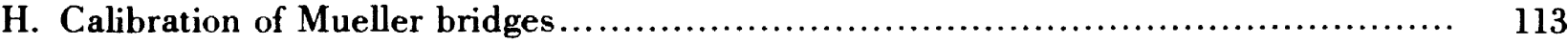

I. Calibration of the linearity of a potentiometer................................... 122

J. Guide for obtaining the calibration of standard platinum resistance thermometers... 124 


\title{
Platinum Resistance Thermometry
}

\author{
John L. Riddle, George T. Furukawa, and Harmon H. Plumb
}

\begin{abstract}
The monograph describes the methods and equipment employed at the National Bureau of Standards for calibrating standard platinum resistance thermometers (SPRT) on the International Practical Temperature Scale of 1968 (IPTS-68). The text of the scale is clarified and characteristics of the scale are described. Several designs of SPRT's are shown and discussed in the light of the requirements and recommendations of the text of the IPTS-68. Possible sources of error such as those due to the internal and external self-heating effects and the immersion characteristics of SPRT's are described in detail. Precautions and limitations for the mechanical and thermal treatment and for the shipment of SPRT's are indicated, and a guide is given for those desiring the thermometer calibration services of NBS. The description of equipment employed at the National Bureau of Standards for maintaining the IPTS-68 includes the triple point of water cell, tin point cell, zinc point cell, oxygen normal boiling point comparison cryostat, the 13 to $90 \mathrm{~K}$ comparison cryostat, and the reference SPRT's upon which the NBS-IPTS-68 in the region 13 to $90 \mathrm{~K}$ is based. Methods are given for calculating temperatures from the calibration data and observed resistances; the propagation of calibration errors is discussed. Supplenental information given in the Appendices includes the authorized English version of the text of the IPTS -68 , tabular values of the reference function used below $0^{\circ} \mathrm{C}$, tabular values of the differences between IPTS-68 and IPTS-48, analysis of the first derivatives at $0{ }^{\circ} \mathrm{C}$ of the IPTS-68 formulations, methods for calibrating potentiometers and Mueller bridges, and the derivation of the coefficients used in the analysis of error propagation.
\end{abstract}

Key words: Calibration; caljbration errors; cryostat; fixed points; freezing points; International Practical Temperature Scale; platinum resistarce thermometer; thermodynamic temperature scale; thermometry; triple point.

\section{Introduction}

The National Bureau of Standards (NBS) has the responsibility to establish, maintain, and develop the standards for physical measurements neressary for the nation's industrial and scientific progress and, in cooperation with other national laboratories, to establish international uniformity of the basic physical quantitites. Dissemination of these standards and the methodology of measurements is accomplished by appropriate publications, consultation, and calibration services. One of the important activities is the realization, reproduction, and transmission of the International Practical Temperature Scale of 1968 (IPTS-68), the present basis for assigning uniform values of temperature. Standard platinum resistance thermometers are the prescribed interpolation instruments for realizing the IPTS-68 from 13.8 to $904 \mathrm{~K}$; they also serve as reference standards for calibrating many other types of thermometers, e.g., liquidin-glass thermometers. Thus standard platinum resistance thermometers are the base for an extremely large fraction of the temperature measurements made in science and technology.
The information presented in this monograph is intended for those who use standard platinum resistance thermometers, who wish to submit such thermometers to the NBS for calibration, or who desire information that will aid in establishing local calibration facilities. Good practice in the use of these thermometers is emphasized to assist in the realization of accuracy that is commensurate with their capability, as well as to point out their inherent limitations. This monograph describes the calibration of standard platinum resistance thermometers at the NBS including the equipment, techniques, and procedures. The discussion of the IPTS-68, the defining fixed points, the interpolation formulae, the design of standard platinum resistance thermometers, and pertinent procedures which lead to "state of the art" measurements are presented in considerable detail.

\section{Background and Basic Concepts}

The development of the platinum resistance thermometer resulted in an internationally acceptable practical temperature scale defined to give 
values of temperatures close to those on the thermodynamic scale. The birth of platinum resistance thermometers as useful precision instruments occurred in 1887 when $\mathrm{H}$. L. Callendar $[16]^{t}$ reported that platinum resistance thermometers exhibited the prerequisite stability and reproducibility if they were properly constructed and treated with sufficient care. In the next four decades the platinum resistance thermometers gained such wide acceptance that their use was proposed and adopted by the Comite International des Poids et Measures in 1927 in defining values on a practical scale of temperature, the first International Temperature Scale [15]. Since that time improvements in the purity of platinum and other materials of construction, in thermometer design, and in calibration techniques have yielded improvements in the precision, accuracy, and range of temperatures that can be measured with platinum resistance thermometers. The international scale has been redefined both to take advantage of these improvements and to bring the scale more nearly into agreement with the thermodynamic scale.

\subsection{Thermodynamic Temperature Scale}

Temperature scales based on functions that can be derived from the first and second laws of thermodynamics, and hence that give values of temperature consistent with the entire system of logic ("thermodynamics") derivable from these laws. are said to be thermodynamic scales. The present thermodynamic scale was established in 1954 by assigning the value 273.16 kelvins to the triple point of water. [17] On the Kelvin thermodynamic scale, the values of temperature and the designation of the appropriate units and symbols have been adopted [51] as follows: The unit of measurement on this thermodynamic scale is the kelvin, indicated by the symbol $\mathrm{K}$. The same name and symbol are also used to denote an interval of temperature. The designation of an interval of temperature by "degrees Celsius" is also permitted. The symbol of the value of temperature on the Kelvin thermodynamic scale is $T$. The Celsius thermodynamic scale is defined by the equation: $t=T-273.15 \mathrm{~K}$. The zero of the Celsius scale is $0.01 \mathrm{~K}$ below the triple point of water. (Although the value of the temperature of the ice point is close to and can be taken for all practical purposes to be $0^{\circ} \mathrm{C}$, the value of the ice point is no longer defined as $0^{\circ} \mathrm{C}$.) The unit of a Celsius thermodynamic temperature is the degree Celsius, symbol ${ }^{\circ} \mathrm{C}$, which is equal to the unit of temperature on the Kelvin scale. The symbols of the values of temperature, $t$ and $T$, contain the unit of temperature ${ }^{\circ} \mathrm{C}$ and $\mathrm{K}$, respectively.

'Figures in brackets indicate the literature references on page 50.

\subsection{Practical Temperature Scales}

Accurate measurements of values of temperature on the thermodynamic scale are fraught with experimental difficulties, yet all values of temperature should be referable to the Kelvin thermodynamic scale. "Practical" temperature scales are intended to give values of temperature that are comparatively easily reproduced and, therefore, utilitarian, but most are based on functions that are not related to the first and second laws of thermodynamics. In contrast, vapor pressure scales, e.g., that of ${ }^{4} \mathrm{He}$ [13], are based on the laws of thermodynamics as is also the radiation pyrometry scale. Because many physical laws are based on thermodynamic temperatures, the values of temperature on any practical scale should be as close to the thermodynamic scale as is possible or the differences between a practical scale and the thermodynamic scale should be appropriately documented so that conversions from the practical scale to the thermodynamic temperature scale are possible.

Many practical scales have been used in the last fifty years, but the only scales employing platinum resistance thermometers that have had widespread use in the United States were those defined and sanctioned by either the International Committee of Weights and Measures or the National Bureau of Standards. Successive scales that were defined by the International Committee of Weights and Measures are the International Temperature Scale of 1927 (ITS-27) [15], the International Temperature Scale of 1948 (ITS-48) [46], and the International Practical Temperature Scale of 1948 with the text revision of 1960 (IPTS48) [48]. The differences in the definition of these scales primarily arose from steps to improve the reproducibility of the scale and the values of temperature between -182.97 and $630.5{ }^{\circ} \mathrm{C}$ remained essentially unchanged - the changes in the values of temperature were less than the uncertainty of the values on the scale at the time. These differences are summarized in Appendix B.

Because the ITS-27 [15] extended only $7{ }^{\circ} \mathrm{C}$ below the normal boiling point of oxygen $(-182.962$ ${ }^{\circ} \mathrm{C}$ ), the National Bureau of Standards was motivated to develop a practical scale from the oxygen point down to approximately $11 K[28]$. This scale has been variously referred to as the NBS provisional scale, the Hoge and Brickwedde scale, and most recently as the NBS-39 scale; directly related to this scale is the NBS-55 scale $\left(T_{\mathrm{NBS}-55}\right.$ $=T_{\text {NBS-39 }}-0.01 \mathrm{~K}$ ) [50]. These NBS scales have had widespread use but, together with the previously mentioned international scales, have now been supplanted by the International Practical Temperature Scale of 1968 (IPTS-68) [51] (see Appendix A for the complete text of IPTS -68 and Appendix C for the difference in values of temperature).

The text of the IPTS-68 introduced the first major changes in the international practical scale 
since 1927. Changes were made to extend the range of the scale down to $13.81 \mathrm{~K}$ and to enhance its reproducibility as well as to improve its agreement with the thermodynamic scale. The changes in the values of temperature from the IPTS -48 to the IPTS -68 are tabulated in Appendix C; included in this appendix is a tabulation of the differences between the IPTS -68 and the NBS-55 scale which it replaced in the United States of America. Temperatures on the IPTS 68 may be expressed in either kelvins or degrees Celsius. The symbols and units of the international practical scale are like those described earlier in this section for use with the thermodynamic scale. The symbol for the value of temperature and for the unit of temperature on the International Practical Kelvin Scale are $T_{68}$ and $K$ (Int. 1968), respectively. For the International Practical Celsius Scale they are correspondingly, $t_{68}$ and ${ }^{\circ} \mathrm{C}$ (Int. 1968). The relation between $T_{68}$ and $t_{68}$ is

$$
t_{68}=T_{68}-273.15 \mathrm{~K}
$$

The subscripts or parenthetical parts of the designation need not be used if it is certain that no confusion will result from their omission.

In the text of the IPTS-1968, platinum resistance thermometers are defined as standard interpolation instruments for realizing the scale from -259.34 to $630.74{ }^{\circ} \mathrm{C}$ (or from 13.81 to $903.89 \mathrm{~K}$ ). The resistance values (or ratios of resistance values, $R(t) / R(0)$, where $R(t)$ is the resistance at temperature $t$ and $R(0)$ the resistance at $0{ }^{\circ} \mathrm{C}$ ) of any particular standard platinum resistance thermometer are related to the values of temperature with specified formulae. The constants of the formulae are determined from the resistance values of the thermometer at stated defining fixed points and, usually, from the derivatives of the formula specified for the temperature range immediately above. [Hereafter, platinum resistance thermometers that meet the following specifications, based primarily on the text of the IPTS -68 , will be referred to as standard platinum resistance thermometers and abbreviated as SPRT: The platinum resistor shall be very pure annealed platinum supported in such a manner that the resistor remains as strain-free as practical; the value of $R(100) / R(0)$ shall not be less than 1.3925; the stability of the thermometer shall be such that, when the thermometer is subjected to thermal cycling similar to that encountered in the normal process of calibrating it, the value of $R(0)$ does not change by more than $4 \times 10^{-6} R(0)$; the platinum resistor shall be constructed as a four-lead element and both the resistor and its leads shall be insulated in such a manner that the measured resistance of the platinum resistor is not affected by the insulator more than about $4 \times 10^{-7} R(0)$ at the temperatures of calibration or use; and the four-lead resistor shall be hermetically sealed in a protective sheath. (A SPRT that has a $R(0)$ value of about $25.5 \Omega$ will be referred to as a $25-\Omega$ SPRT.)] The text of the scale assigns an exact numerical value of temperature to each of the defining fixed points. The defining points and their assigned values are listed in table 1 . The realization of the fixed points

TABLE 1. Defining fuxed points of the IPTS $-68^{\text {a }}$

\begin{tabular}{|c|c|c|c|}
\hline \multirow[t]{2}{*}{ Equilibrium state } & \multicolumn{2}{|c|}{$\begin{array}{c}\text { Assigned value of } \\
\text { International Practical } \\
\text { Temperature }\end{array}$} & \multirow[t]{2}{*}{$\boldsymbol{W}^{*}$} \\
\hline & $T_{68}(\mathrm{~K})$ & $t_{68}\left({ }^{\circ} \mathrm{C}\right)$ & \\
\hline $\begin{array}{l}\text { Equilibrium between the solid, liquid and vapor phases of equilibrium hydrogen (triple } \\
\text { point of equilibrium hydrogen) } \ldots \ldots \ldots \ldots \ldots \ldots \ldots\end{array}$ & 13.81 & -259.34 & d0.0014 1207 \\
\hline $\begin{array}{l}\text { Equilibrium between the liquid and vapor phases of equilibrium hydrogen at a pressure } \\
\text { of } 33330.6 \mathrm{~N} / \mathrm{m}^{2}(25 / 76 \text { standard atmosphere }) \ldots \ldots \ldots \ldots \ldots \ldots \ldots \ldots \ldots \ldots \ldots \ldots \ldots \ldots \ldots \ldots \ldots \ldots \ldots \ldots \ldots \ldots\end{array}$ & 17.042 & -256.108 & d0.0025 3445 \\
\hline $\begin{array}{l}\text { Equilibrium between the liquid and vapor phases of equilibrium hydrogen (boiling point } \\
\text { of equilibrium hydrogen) }\end{array}$ & 20.28 & -252.87 & 0.00448517 \\
\hline Equilibrium between the liquid and vapor phases of neon (boiling point of neon)........... & 27.102 & -246.048 & 0.01221272 \\
\hline Equilibrium between the solid, liquid and vapor phases of oxygen (triple point of oxygen). & 54.361 & -218.789 & ${ }^{d} 0.09197253$ \\
\hline Equilibrium between the liquid and vapor phases of oxygen (boiling point of oxygen)....... & 90.188 & -182.962 & d0.24379911 \\
\hline Equilibrium between the solid, liquid and vapor phases of water (triple point of water) ${ }^{\mathrm{c}}$. & 273.16 & 0.01 & \\
\hline Equilibrium between the liquid and vapor phases of water (boiling point of water) ${ }^{\mathrm{b}, \mathrm{c}} \ldots \ldots$ & 373.15 & 100 & 1.39259668 \\
\hline Equilibrium between the solid and liquid phases of zinc (freezing point of zinc). & 692.73 & 419.58 & 2.56848557 \\
\hline
\end{tabular}

\footnotetext{
a Except for the triple points and one equilibrium hydrogen point ( $17.042 \mathrm{~K}$ ) the assigned values of temperature are for equilibrium states at a pressure $p_{0}=1$ standard at mosphere $\left(101325 \mathrm{~N} / \mathrm{m}^{2}\right)$

b The equilibrium state between the solid and liquid phases of tin (freezing point of tin) has the assigned value of $t_{6 \times}=231.9681^{\circ} \mathrm{C}$ and may be used as an alternative to the boiling point of water. At this value of temperature $W^{*}=1.89257109 .^{d}$ The value of $t^{\prime}=231.929163{ }^{\circ} \mathrm{C}$.

'The water used should have the isotopic composition of ocean water (see sec. III.4, Appendix A).

d This value is slightly different from that given in the text of the IPTS-68 (see Appendix A).
} 
and the use of interpolation formula are discussed in detail in sections 7 and 6 , respectively.

The IPTS -68 is defined by the text of the scale. However, even if perfect experimental work were possible, the values of temperature would not be unique because of a lack of uniqueness in the definition of the scale itself. For example, the text of the scale specifies the minimum quality of the platinum to be used in the SPRT; yet different samples of suitable platinum may not give exactly the same value of a temperature between defining fixed points. The different values of temperature obtained would, however, be in accordance with IPTS 68 because both samples of platinum are within the definition of the scale. Another source of difference is the variation in the realization of the fixed points where small differences in the purity of the fixed-point samples could easily occur. This spread of temperatures (the "legal" spread of the scale) is the result of the looseness of the definition of the scale; it is small compared to the usual errors of experimental measurement, but does exist and is a practical necessity if the physical properties of real materials are to be used in realizing the scale.

\section{Platinum Resistance Thermometer Construction}

This discussion is primarily directed toward thermometers that are suitable as defining standards on the IPTS-68; however, many of the techniques described are applicable to any resistance thermometer. To be suitable as a SPRT as described in the text of the scale (Appendix A) the resistor must be made of platinum of sufficient purity that the finished thermometer will have a value of $R(100) / R(0)$ not less than 1.3925 or $\alpha$, defined as $(R(100)-R(0)) / 100 R(0)$, not less than 0.003925 . This requirement provides a scale that is more closely bounded and is not unreasonable since platinum wire of sufficient purity to yield the above ratio is now produced in several countries. The typical SPRT has an ice-point resistance of about $25.5 \Omega$ and its resistor is wound from about $61 \mathrm{~cm}$ of $0.075 \mathrm{~mm}$ wire. The wire is obtained "hard drawn," as it is somewhat easier to handle in this condition, but it is annealed after the resistor is formed. Although wires between $0.013 \mathrm{~mm}$ and 0.13 $\mathrm{mm}$ diameter are commonly used in industrial platinum thermometers, experience shows that finer wires tend to have lower values of $R(100) / R(0)$ (implying the presence of inore impurities or strains).

The insulation material that supports the resistor and leads must not contaminate the platinum during the annealing of the assembled thermometer nor when subjected for extended periods of time to temperatures to which thermometer is normally exposed. The insulation resistance between the leads must be greater than $5 \times 10^{9} \Omega$ at $500{ }^{\circ} \mathrm{C}$ if the error introduced by insulation leakage in the leads of a $25-\Omega$ thermometer is to be less than the equivalent of $1 \mu \Omega$. For SPRT's the most commonly used insulation is mica. The primary difficulties in the use of mica are the evolution of water vapor at high temperatures and the presence of the iron oxide impurity which, if reduced, leaves free iron that will contaminate the platinum. The most usual mica is muscovite $\left(\mathrm{H}_{2} \mathrm{KAl}_{3}\left(\mathrm{SiO}_{4}\right)_{3}\right)$, sometimes called India or ruby mica; it contains about 5 percent water of crystallization. The dehydration starts at approximately $540{ }^{\circ} \mathrm{C}[41]$ and not only causes mechanical deterioration of the mica but supplies free water vapor that reduces the insulation resistance. Phlogopite mica $\left(\mathrm{H}_{2} \mathrm{KMg}_{3} \mathrm{Al}\left(\mathrm{SiO}_{4}\right)_{3}\right)$ or "amber" mica is used for thermometers that are expected to function up to $630^{\circ} \mathrm{C}$. Although the room temperature resistivity of phlogopite mica is slightly lower than that of muscovite, it does not begin to dehydrate until well above $700^{\circ} \mathrm{C}$ [4.1]

Some designs employ very high-purity alumina or synthetic sapphire insulation (see fig. 1) $[1,18,23$, 52]. Fused silica has also been used [18]. Although the properties of these materials are superior to mica at high temperatures, the materials are more difficult to fabricate and, additionally, they must be

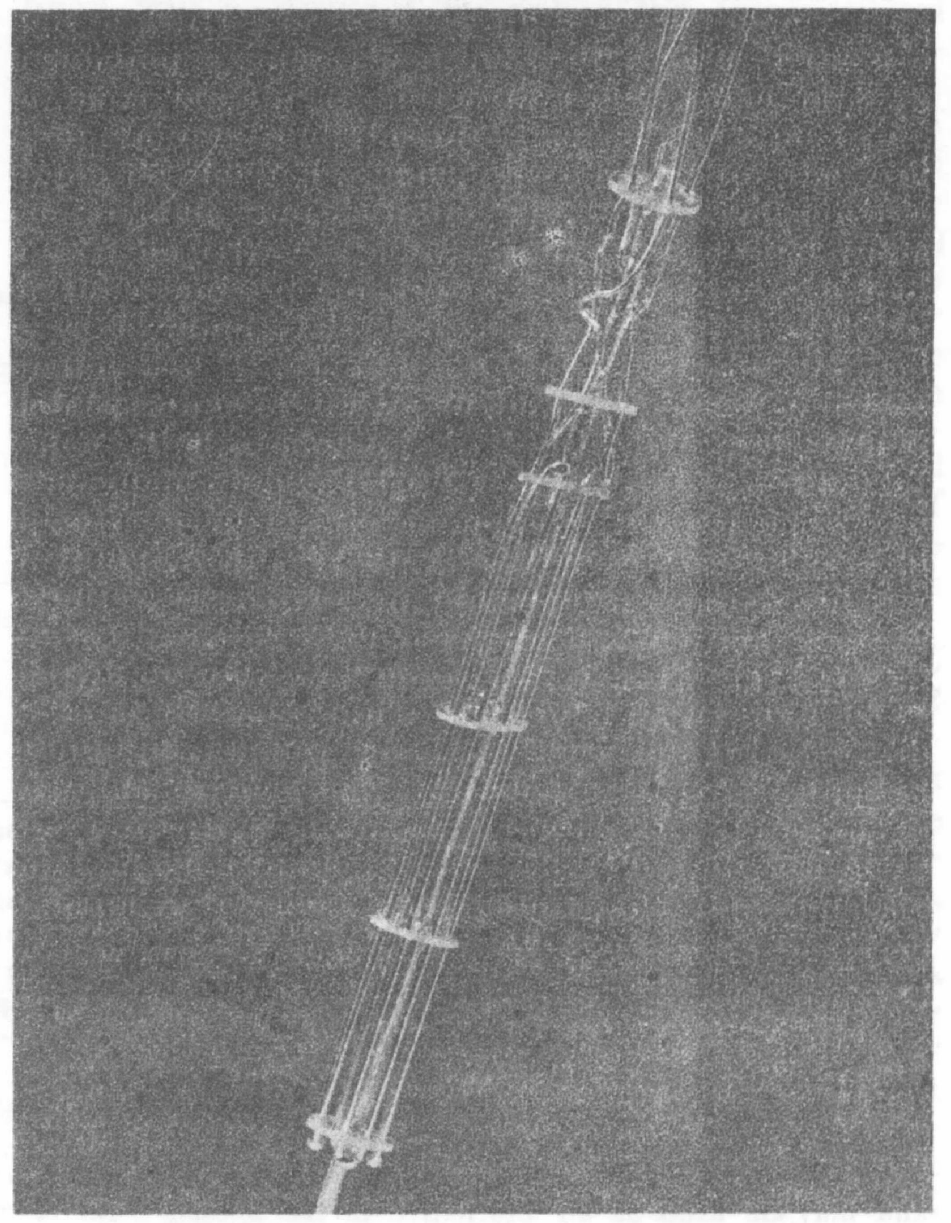

FiguRE 1. Synthetic sapphire form for thermometer.

Synthetic sapphire disks are employed to keep the platinum wires separated in a "bird-cage" type SPRT. A centrally located, heavier gage platinum wire maintains the spacings between the disks [23]. 


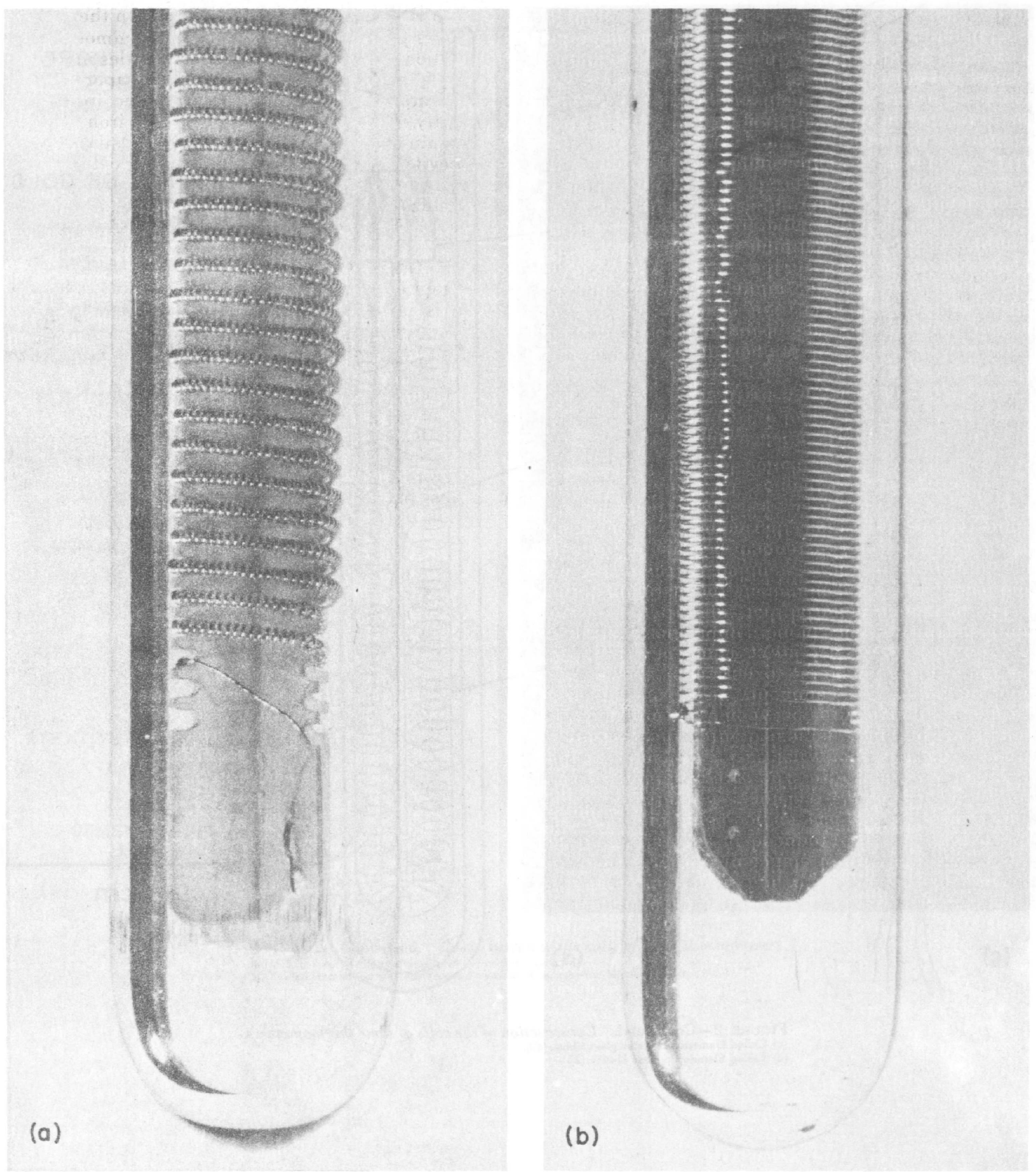

FiguRE 2. Construction of the coils of some thermometers.

(a) Coiled filament on mica cross [34].

(b) Single layer filament on mica cross [47]. 

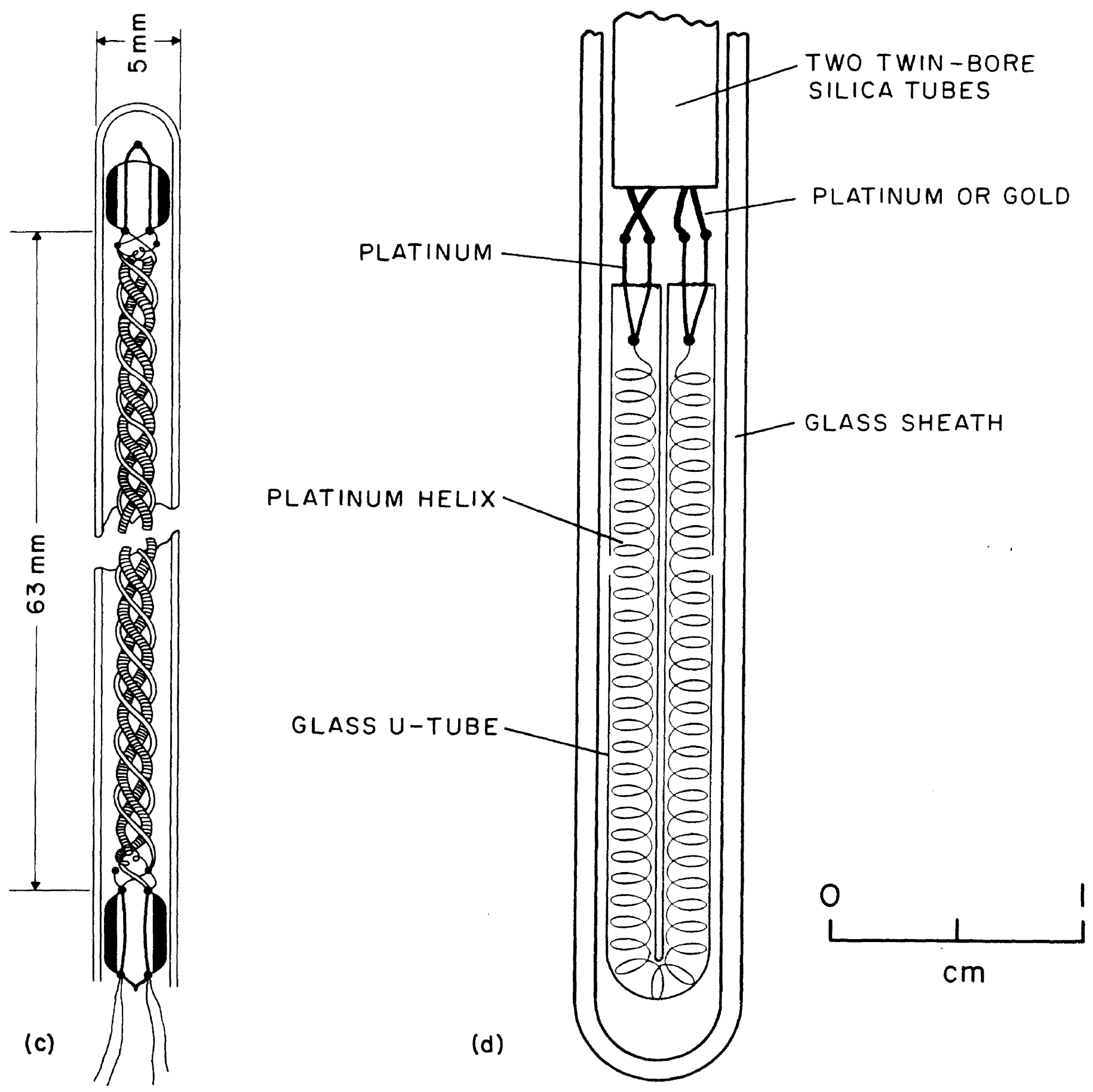

FIGURE 2-Continued. Construction of the coils of some thermometers.

(c) Coiled filament on twisted glass ribbon [49].

(d) Coiled filament in glass U-tube [2]. 

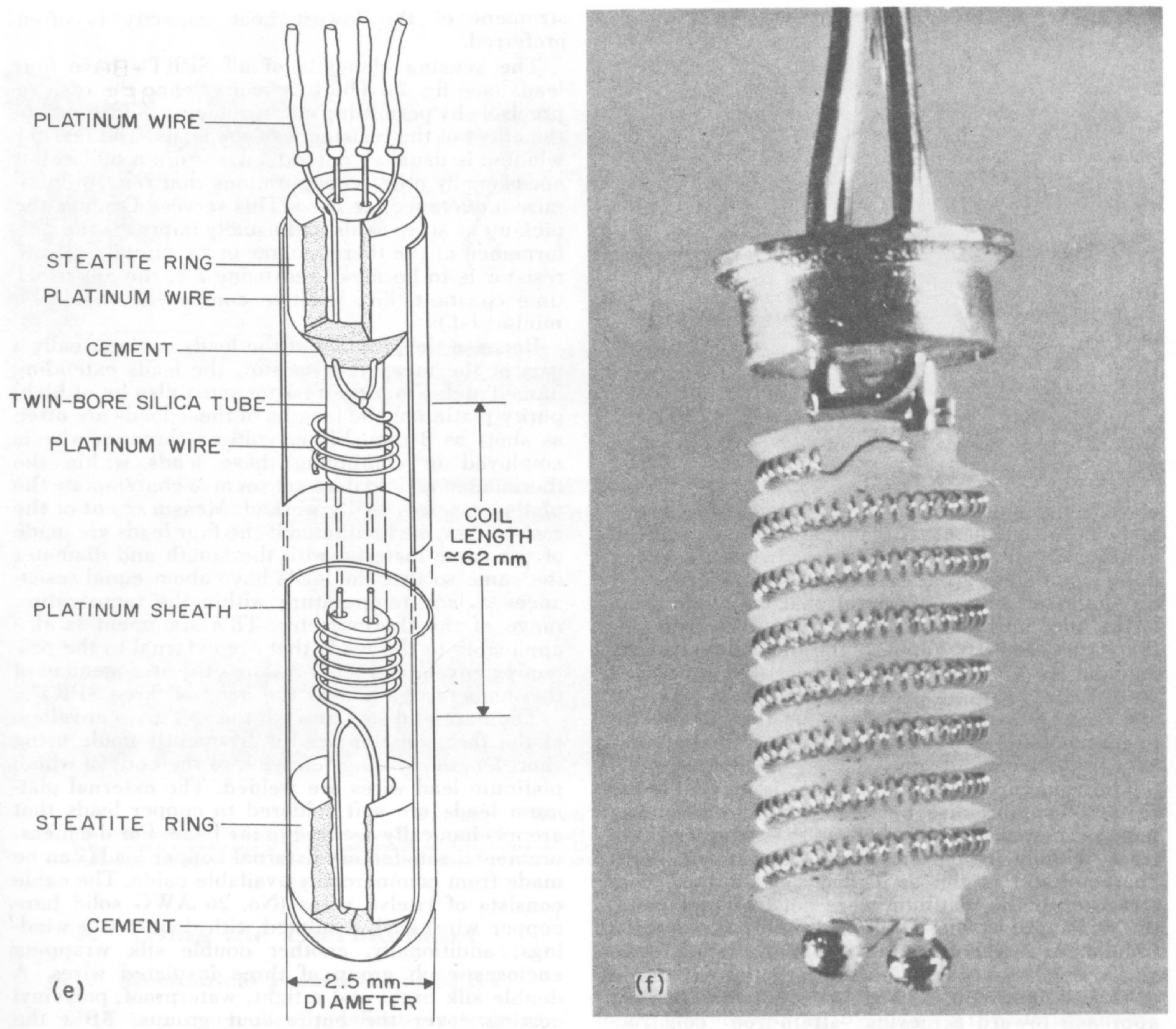

FIgURE 2-Continued. Construction of the coils of some thermometers.

(e) Single layer filament around twin-bore silica tubing [3].

(f) Coiled filament on helically grooved ceramic. (Courtesy the Minco Products, Inc.) 


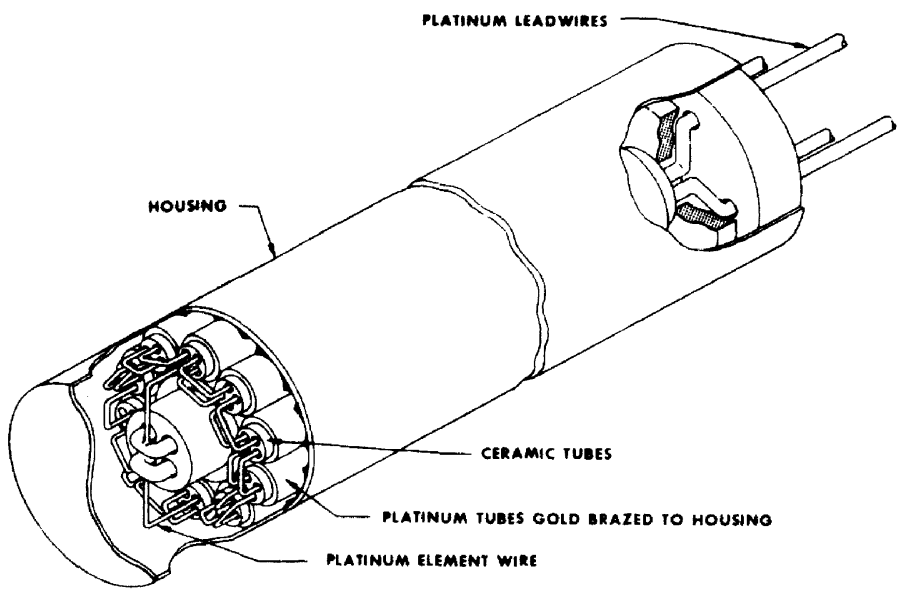

FIGURE 2-Continued. Construction of the coils of some thermometers.

(g) Filament threaded through four-hole ceramic tubes. (Courtesy the Rosemount Engineering Company.)

of very high purity because migration of impurities and the consequent contamination occurs much more easily at high temperatures. In cutting either mica or alumina great care must be taken to avoid or eliminate traces of metal that originate from either the cutting tool or from the metal clamps that are used to support the insulation during machining. A "carbide tipped" tool should be used for the cutting process. Unfortunately, attempts to remove the metal chemically from the mica result in contaminating the mica. Synthetic sapphire, however, can be chemically cleaned after machining.

The configuration of the resistor is inevitably the result of compromise between conflicting requirements. The resistor must be free to expand and contract without constraint from its support. This characteristic is the so called "strain-free" construction. If the platinum were not free to expand, the resistance of the platinum would not only be a function of temperature but would also relate to the strain that results from the differential expansion of the platinum and its support. Seven methods of approach toward achieving "strain-free" construction are illustrated in figure $2[2,3,4,5,34,47]$. Because of the lack of adequate mechanical support, the wire in each of these designs may be strained by acceleration, e.g., shock or vibration. The thermal contact of the resistor with the protecting envelope or sheath is primarily through gas which, even if the gas is mostly helium, is obviously poor compared to the thermal contact that is possible through many solid materials. This poor thermal contact increases the self-heating effect and the response time of the thermometer. The designs shown in figures $2 a .2 b$, and $2 \mathrm{~g}$ suffer less in these respects than the others. On the other hand, for calorimetric work the in- strument of the lowest heat capacity is often preferred.

The sensing elements of all SPRT's have four leads (see fig. 2). The four leads define the resistor precisely by permitting measurements that eliminate the effect of the resistance of the leads. The resistor winding is usually "noninductive," often bifilar, but occasionally other configurations that tend to minimize inductance are used. This serves to reduce the pick-up of stray fields and usually improves the performance of the thermometer in a-c circuits. (If the resistor is to be measured using a-c, the electrical time constant, i.e., reactive component should be minimized.)

Because the junction of the leads is electrically a part of the measured resistor, the leads extending immediately from the resistor must also be of highpurity platinum; the lengths of these leads are often as short as $8 \mathrm{~mm}$. Either gold or platinum wire is employed in continuing these leads within the thermometer. Gold does not seem to contaminate the platinum and is easily worked. Measurement of the resistor may be facilitated if the four leads are made of the same material with the length and diameter the same so that the leads have about equal resistances at any temperature within the temperature range of the thermometer. This statement is also applicable to the leads that are external to the protecting envelope. Figure 3 shows the arrangement of thermometer leads near the head of three SPRT's.

The hermetic seal through the soft glass envelope at the thermometer head is frequently made using short lengths of tungsten wire, to the ends of which platinum lead wires are welded. The external platinum leads are soft soldered to copper leads that are mechanically secured to the head. F or d-c measurements, satisfactory external copper leads can be made from commercially available cable. The cable consists of twelve wires (No. 26 AWG solid bare copper wire) each insulated with double silk windings; additionally, another double silk wrapping encloses each group of three insulated wires. A double silk braid and a tight, waterproof, polyvinyl coating cover the entire four groups. After the appropriate end insulation is removed, the ends of the three wires in each group are twisted together to form a lead to which a lug is soldered. Stranded leads which do not have individually insulated strands should not be used, as the breakage of a single strand may cause "noise" in the resistance thermometer circuits which is difficult to locate and eliminate. The leads external to small capsule type thermometers are usually solid copper wires, although wires of other materials such as manganin are sometimes used; the leads to the thermometer must be placed in good thermal contact with the system to be measured by the capsule thermometer. 


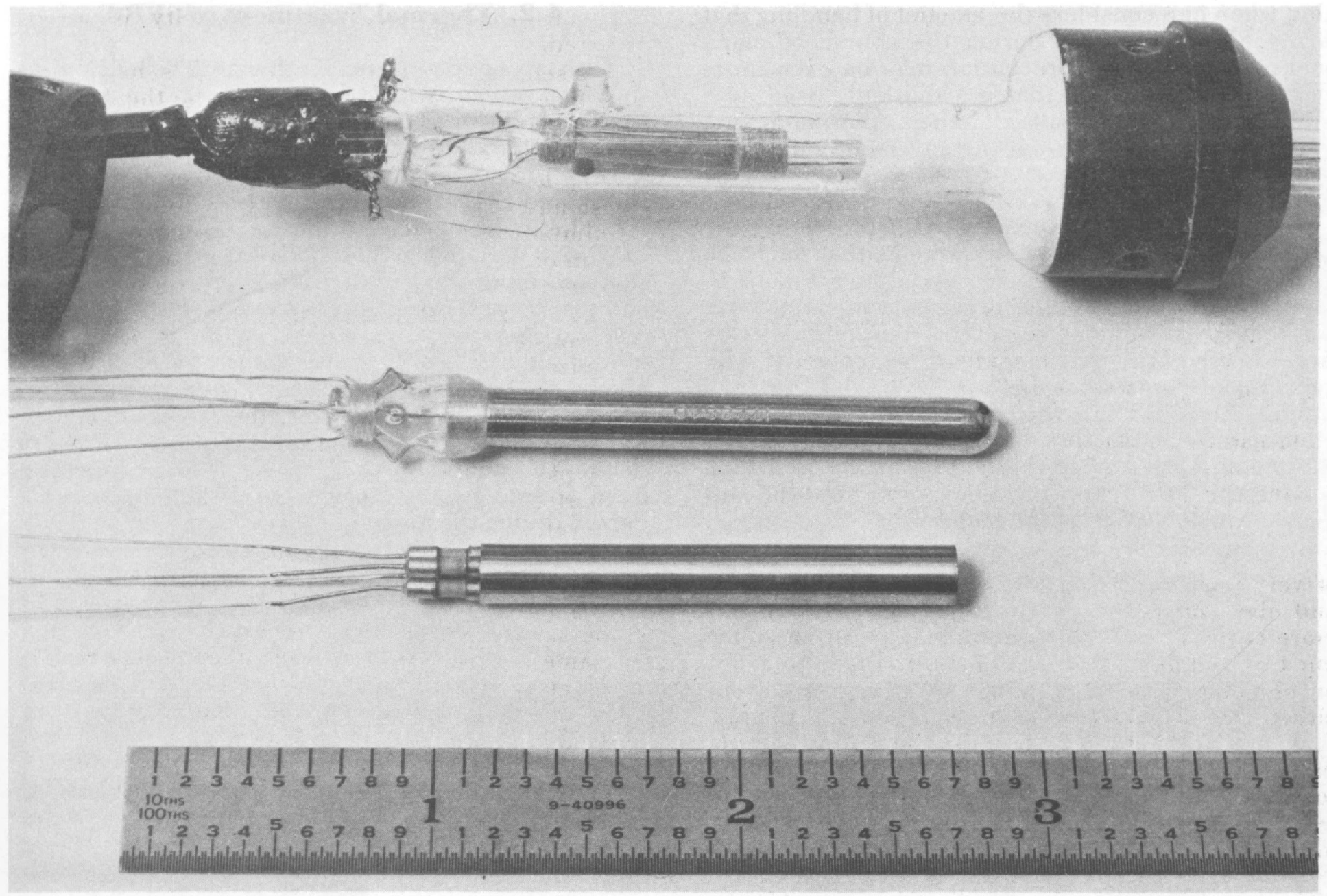

FigURE 3. "Heads" of three SPRT's.

The upper head is that of a long stem thermometer. The internal leads are brought out through hermetic seals and are connected to external copper leads at the left. In the center is a capsule type SPRT with leads brought out through glass seals. Capsule body is platinum.

The bottom capsule type SPRT shows the thermometer leads brought out through individual metal-ceramic-metal type seals. Capsule body is stainless steel.

\section{Using the Thermometer}

\subsection{Mechanical Treatment of SPRT}

A SPRT is a mechanically delicate instrument. As discussed in the section on thermometer construction, the platinum wire cannot be rigidly supported and at the same time be free to expand and contract with temperature changes. Shock, vibration, or any other form of acceleration may cause the wire to bend between and around its supports, thus producing strains that change its temperature-resistance characteristics. Strains in the platinum resistor normally will increase the resistance and decrease the value of $\alpha$. If a tap of a thermometer with glass sheath is hard enough to be audible, but still not cause breakage, the action will typically increase the triple-point resistance of a $25 \Omega$ SPRT by an amount between 1 and $100 \mu \Omega$. (A change of $100 \mu \Omega$ is equivalent to $0.001{ }^{\circ} \mathrm{C}$ in temperature.) Thermometers that have received repeated shocks of this kind through rough handling over a one year period have increased in resistance at the triple point of water by an amount equivalent to $0.1{ }^{\circ} \mathrm{C}$. Similar changes may be caused by using the thermometer in a bath that transmits vibrations to the thermometer or by shipping the thermometer not suitably packed.

It is preferable to "hand carry" the thermometer to maintain the integrity of its calibration. If the thermometer must be shipped, it should first be placed in a rigid and moderately massive container that has been lined with material which softly conforms to the thermometer and protects it from mechanical shocks or vibrations. This container should then be packed in an appreciably larger box with generous room on all sides for soft packing material that will absorb or attenuate the shocks that might occur during shipment. Two reasonable package configurations are shown in figures 4 and 5 .

In arranging storage for the thermometer in the laboratory, a container should be used that minimizes or eliminates the possibility of "bumping" the thermometer. This is a most reasonable precau- 
tion when one considers the amount of handling that many SPRT's receive during the course of many measurements. This precaution may be even more pertinent for SPRT's that are routinely used as a standard for calibrating other thermometers. Figure 6 shows a storage arrangement employed at NBS.

Care should also be exercised in protecting the thermometer from cumulative shocks that might be received during insertion into apparatus. Figure 18 (sec. 7) shows the simple provisions made at NBS for reducing mechanical shock when thermometers are inserted into triple-point of water cells. The polyethylene plastic tube at the top of the cell guides the SPRT into the thermometer well without "bumping", and the tapered entrance of the aluminum sleeve near the bottom of the well then guides the SPRT into the sleeve and onto the soft polyurethane sponge at the bottom.

\subsection{Thermal Treatment of SPRT}

With exception of specific instances, SPRT's, as they are generally used, are not greatly susceptible to damage from thermal shocks. In the case of capsule type thermometers, the metal to glass seals may be broken by rapid cooling, e.g., when a capsule thermometer that has been at ambient temperature is quickly immersed in liquid nitrogen. Sudden exposure of capsule thermometers to temperatures that are much higher than ambient seldom occurs since they are rarely used above $100^{\circ} \mathrm{C}$. Another specific instance of thermal shock is subjecting long-stem SPRT's to temperatures that are well above $600^{\circ} \mathrm{C}$ and subsequently quenching them. This treatment can mechanically damage the thermometer but, even if no visible damage has been done, the calibration of the thermometer may have been altered by the freezing in of defects that occurred during the quenching [24].

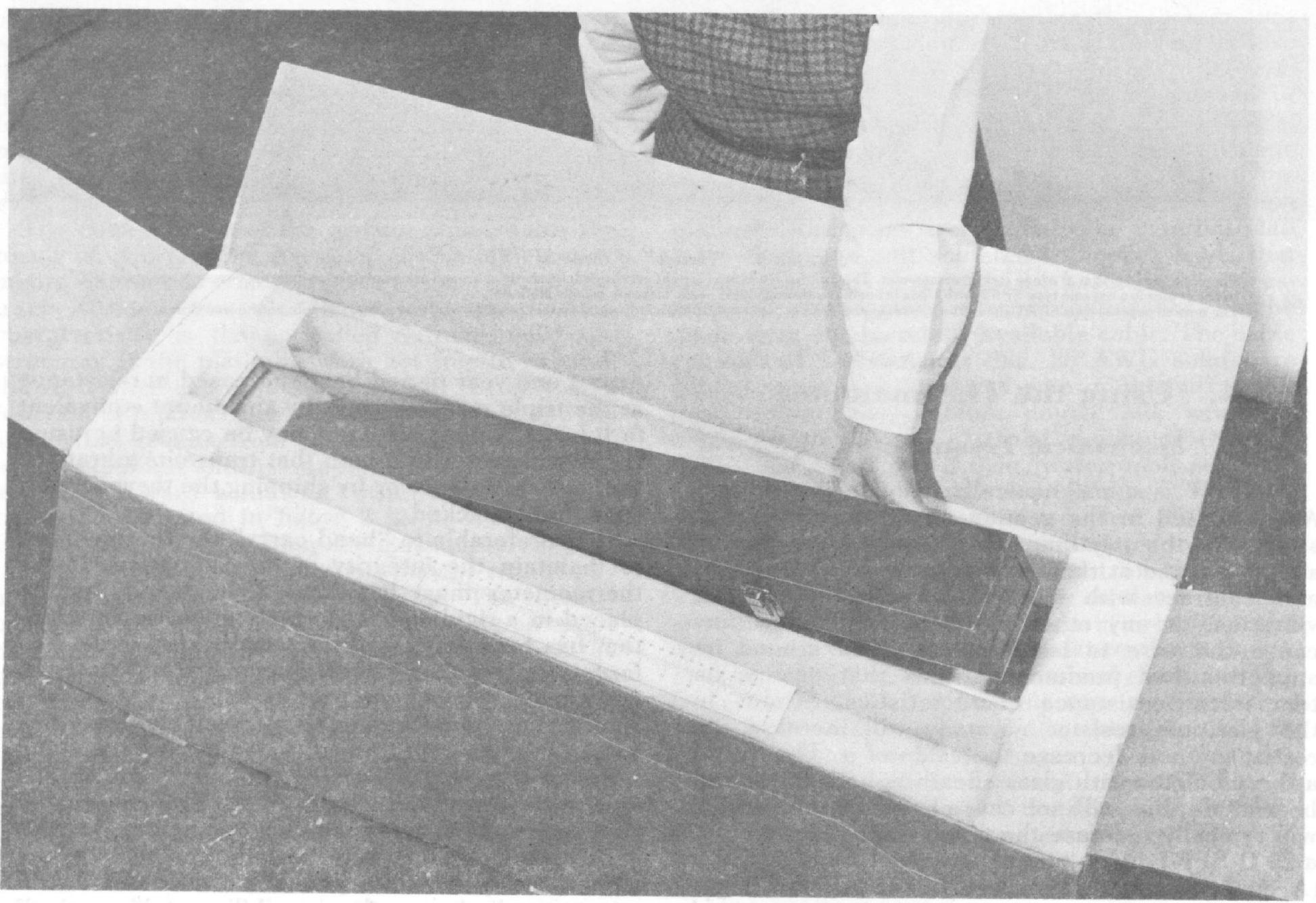

FIGURE 4. A method for packaging a SPRT for shipment.

The metal case contains a SPRT snugly nested in polyurethane foam. The metal case in turn is protected during shipment by a tightly fitted polyurethane foam lined box. 


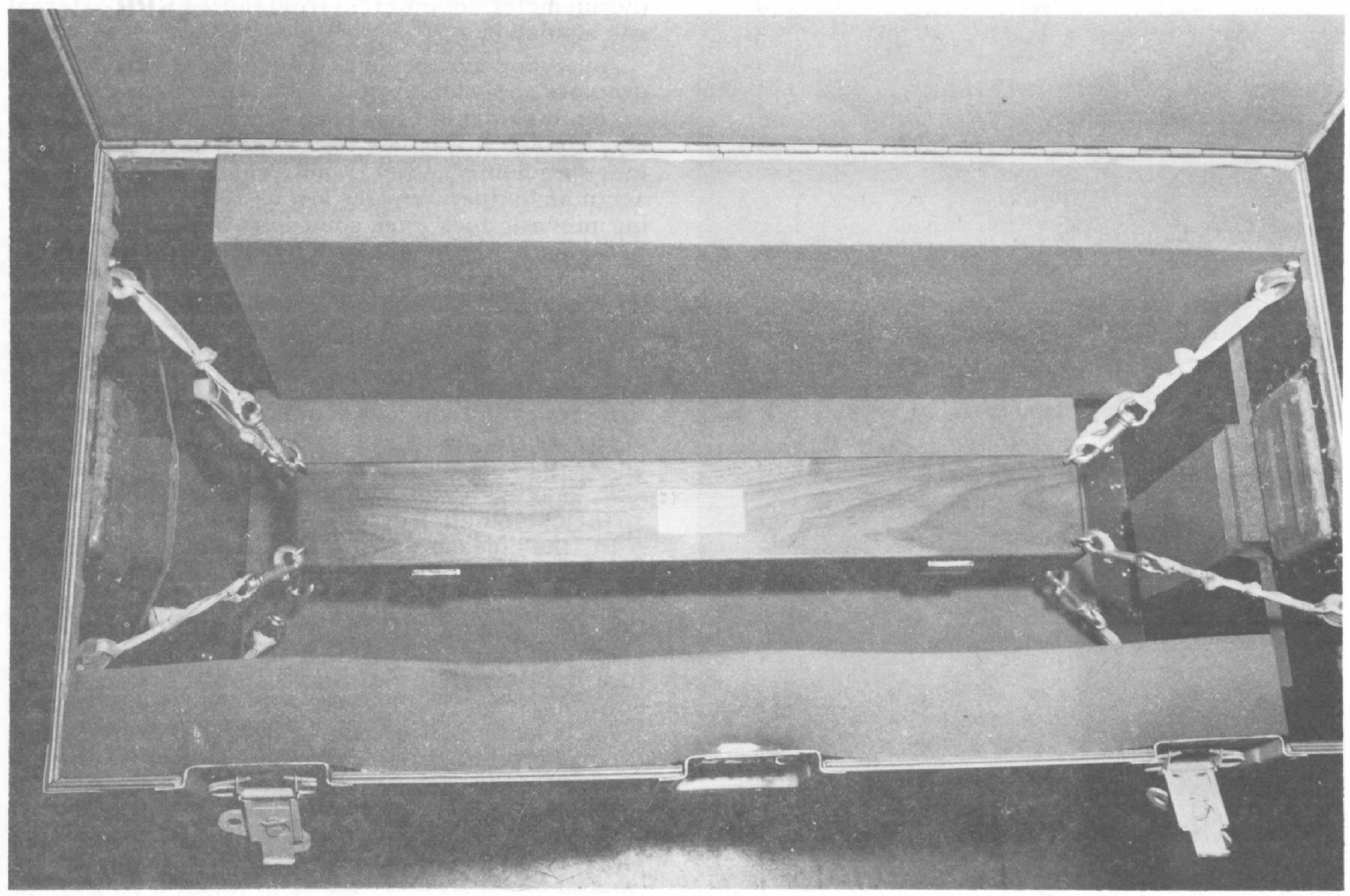

FIGURE 5. Special container for SPRT shipment.

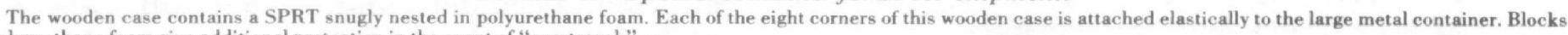
of polyurethane foam give additional protection in the event of "overtravel."

(courtesy the Space Division, North American Rockwell Corporation)

The upper temperature limit of a SPRT is restricted by the softening point of the material of the protecting sheath, the temperature at which the thermometer was annealed before calibration, the evolution of water and other contaminants from the sheath and insulators, and grain growth in the platinum wire. The concomitant changes are a function of both time and temperature and most are predictable in only a qualitative way.

Thermometer sheaths of borosilicate glass soften noticeably at temperatures above $500^{\circ} \mathrm{C}$, e.g., their use at $515^{\circ} \mathrm{C}\left(1000^{\circ} \mathrm{F}\right)$ is limited to a few hours unless they are specially supported to prevent deformation. Fused silica sheaths should be used for measurements above $500^{\circ} \mathrm{C}$. Platinum grain growth has been observed in SPRT's that have been used over a period of several hundred hours at $420^{\circ} \mathrm{C}$. Figure 7, made with an electron microscope, shows in detail a short section of platinum wire from a thermometer that was subjected to such a treatment. Grains which are one to two wire diameters long and as large as the wire in diameter may be clearly seen. The growth of large grains results in a thermometer that is more susceptible to calibration changes caused by mechanical shock; consequently, the thermometer may be considered unstable. The evolution of water within the thermometer sheath at temperatures below $500^{\circ} \mathrm{C}$ has been observed in a few thermometers but seems to be avoidable if sufficient baking and evacuation are furnished during the fabrication of the thermometer. The presence of water may become more conspicuous when the thermometer is cooled, e.g., in a triple point cell. The presence of water, either within the thermometer or on the external insulated leads, will be evidenced during electrical measurements by the kick of the galvanometer when the current through the thermometer is reversed. The kick stems from the polarization of water on the insulation. The decay time (the time for repolarization of water on the insulation) of the galvanometer pulse is usually greater than a minute when moisture exists in the thermometer but is only of a few seconds' duration with moisture on silk insulated leads, indicating that the quantity and physical state of moisture and the effect of moisture on the insulation inside the 


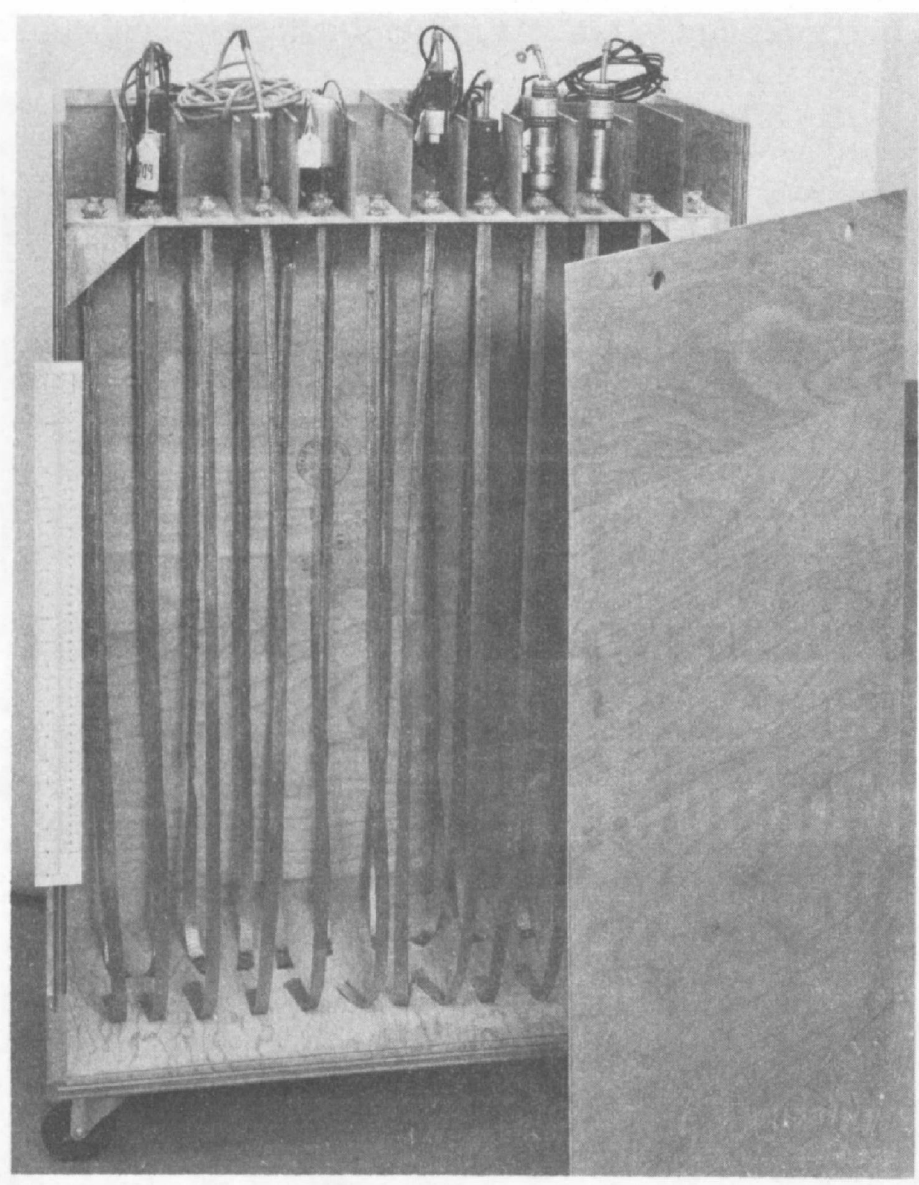

Figure 6. A storage for SPRT's.

The thermometers are stored vertically by slipping into the wire braid sleeves. The cover to the box has been removed only to show the arrangement of the wire braid sleeves. thermometer are different from those on the external silk insulation.

Annealing may occur if the platinum wire was not properly annealed during the manufacturing process or (more likely) if it has been strained by mechanical shock since it was last well annealed. If the strains are sufficiently severe, noticeable annealing will occur at temperatures as low as $100^{\circ} \mathrm{C}$. The annealing process does have somewhat beneficial aspects because the thermometer tends to return toward the metallurgical state that it presumably was in during its previous calibration. (At NBS all long-stem SPRT's are annealed between 470 and $480^{\circ} \mathrm{C}$ for 4 hours, removed from the furnace while still hot, and allowed to cool in air at ambient temperature prior to their calibration). The comparison of the resistance of the thermometer at a fixed point before and after annealing will show a shift downward in resistance and, usually, a shift upward in $R(t) / R(0)$ (see sec. 6 on the computation of temperature).

In the measurements at temperatures above $500{ }^{\circ} \mathrm{C}$ the difficulties discussed above are more likely to occur or be accelerated. More care must be taken in baking and evacuating the thermometer after assembly. For work near the upper temperature limit $\left(630^{\circ} \mathrm{C}\right)$, the SPRT's should have phlogopite mica or fused silica coil forms and a fused silica sheath. Also, care should be exercised to avoid the previously mentioned effect of freezing in of high temperature defects when cooling the thermometer.
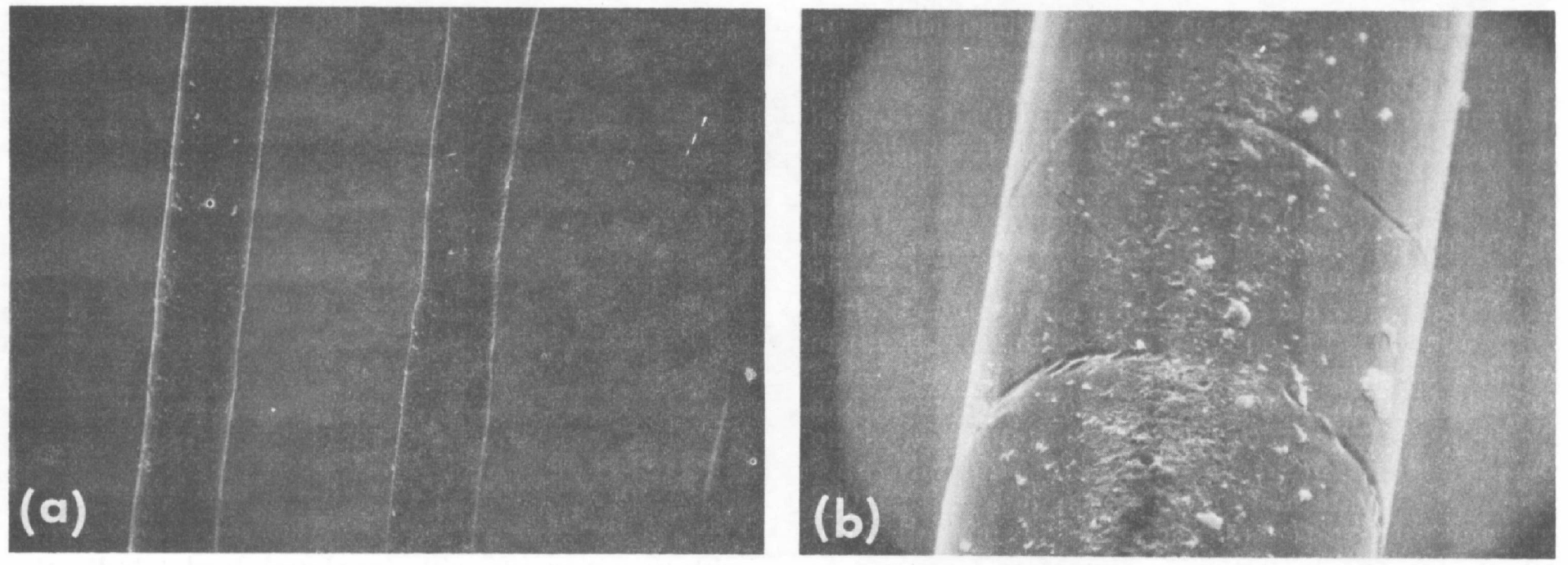

FIGURE 7. Electron microphotograph of platinum thermometer wire subjected to a total of several hundred hours at $420^{\circ} \mathrm{C}$. Magnification: photograph (a) $172 \times$ (b) $850 \times$. The photographs show: (1) grain growth; (2) migration. No attempt has been made to date to identify the white "flecks." 


\subsection{Thermometer Immersion}

A thermometer is sufficiently immersed in the bath when there is no heat flow between the sensor and its environment through the thermometer leads or sheath that extend from the region of sensor. Regrettably, considering the ease with which the adequacy of immersion can be checked, insufficient immersion of a thermometer is still a very common error. The test simply involves a equilibrium temperature measurements at two depths of immersion, while the bath is maintained at very nearly constant temperature. If, after taking into account any change in the temperature of the bath, the temperatures at the different immersions do not agree, an immersion problem exists. Experimentalists are frequently led astray by the erroneous idea that the required immersion is strongly temperature dependent. Figure 8 shows the difference between the bath temperature and the temperature indicated by the thermometer as a function of immersion for two different thermometers. The immersion characteristics are more clearly seen in figure 9 where the same data are given in a semilog plot. A linear relationship between the immersion and the logarithm of the temperature difference is to be expected in simple cases and in practice this is a very useful approximation in the usual region of interest, namely, where the temperature difference is small. Figure $\mathbf{9}$ shows that for thermometer $\mathrm{G}$ in an ice bath, the temperature difference is reduced by a factor of 10 for each $3.3 \mathrm{~cm}$ of immersion. For thermometer $M$, the temperature difference is reduced by a factor of 10 for each $1.4 \mathrm{~cm}$ of immersion. If the error due to immersion is to be less than $0.025 \mathrm{mK}$, the temperature difference must be reduced by six orders of magnitude and, therefore, both thermometers must be immersed in the ice bath by six times the respective amounts cited above, or a total of $19.8 \mathrm{~cm}$ and $8.4 \mathrm{~cm}$, respectively. Even if the temperature difference between the ambient and the bath were only 2.5 degrees the thermometer immersion could be decreased only slightly because, for the same accuracy, a reduction of the temperature difference by five factors of ten is still needed.

The similarity of the radial conductance of heat to or from the thermometer in the bath is important. Figure 10 shows the immersion characteristics of thermometers $G$ and $M$ in a triple-point of water cell rather than an ice bath. The immersion characteristics have changed for both thermometers, but particularly for thermometer M. This is caused primarily by the increase in thermal resistance radially from the thermometer to the ice-water interface of the triple point cell: the sheath of water that surrounds the thermometer within the triple-point cell well and the glass wall of the well contribute to the increased thermal resistance (see also sec. 4.4). The immersion problem would be even worse if the space between the thermometer well and the thermometer were filled with air

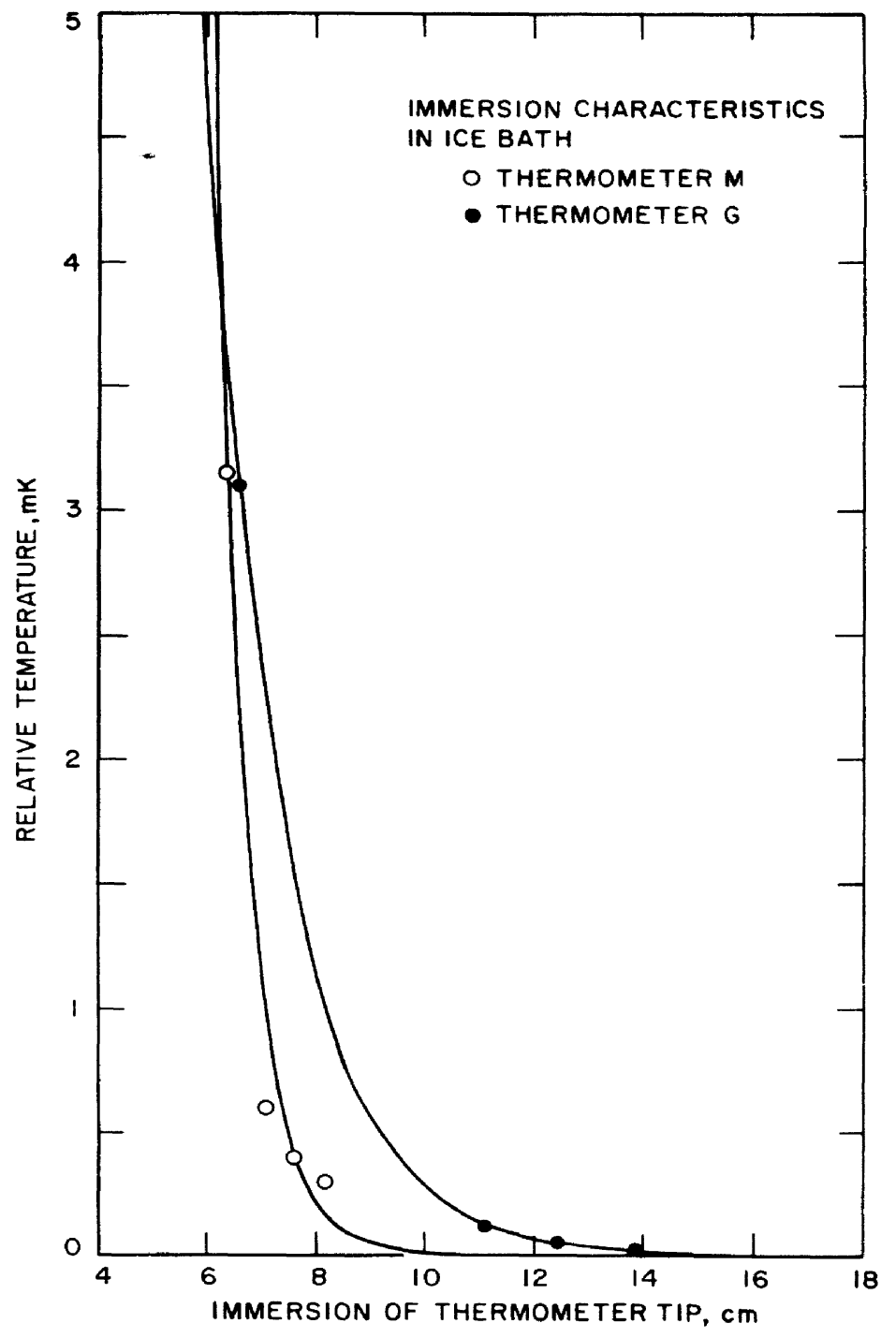

FIGURE 8. Immersion characteristics in an ice bath of two long stem SPRT's with different sheath materials and internal construction.

The plot shows the relative temperature as a funciion of the depth of thermometer immersion.

instead of water. Typical immersion data for thermometers in tin and zinc freezing point cells are given in the discussion of that apparatus (see sec. 7).

\subsection{Heating Effects in SPRT}

The measurement of resistance necessarily involves passing a current through the resistor. The resultant heating that occurs in the resistor and its leads raises their temperature above that of their surroundings until the resistor element attains a temperature sufficiently higher than the surroundings to dissipate the power developed. A typical steady-state profile of the radial temperature distribution caused by a current of $1 \mathrm{~mA}$ flowing in a $25 \Omega$ SPRT is shown in figures 11 and 12 . In figure 


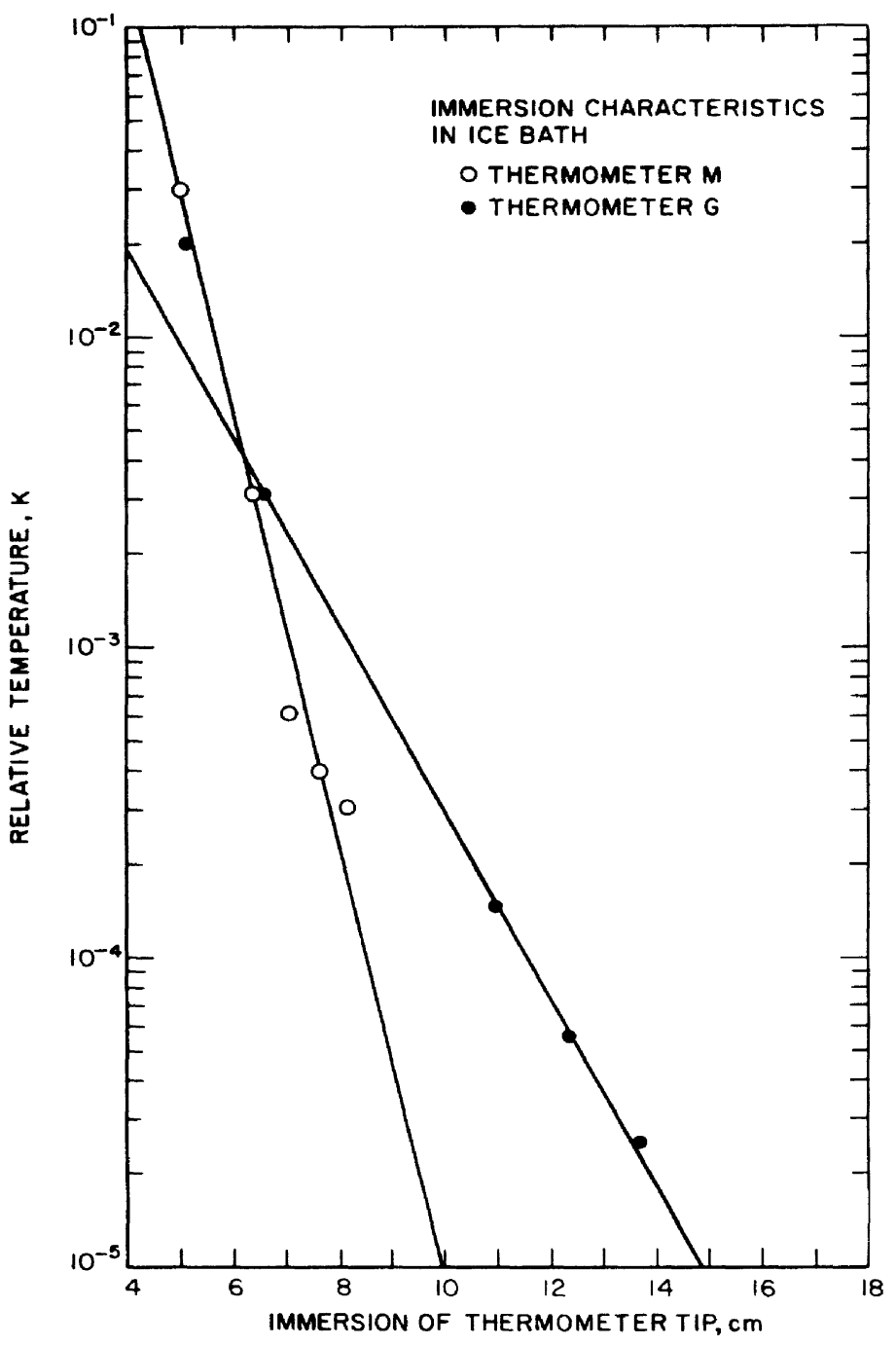

Figure 9. Immersion characteristics in an ice bath of two long stem SPRT's with different sheath materials and internal construction.

The dat a of figure 8 have been replotted to show the linear relationship between the logarithm of the relative temperature and the depth of thermometer immersion.

11 , the internal heating effect of the thermometer, i.e., between the platinum resistor and the outside wall of the protecting sheath, at a given environmental temperature is a function only of the thermometer construction and the current and is, therefore, the same during both calibration and use. This assumes that the thermometer resistor does not move within its protecting sheath. At the ice point, the internal heating effect may be measured by direct immersion in the ice bath and is typically between $0.3 \mathrm{mK} /(\mathrm{mA})^{2}$ and $1.2 \mathrm{mK} /(\mathrm{mA})^{2}$ for a $25 \Omega$ SPRT. If the thermometer is used with the same current that was used during its calibration. the same internal heating effect occurs and no error is introduced in the measurement.

As figure 12 indicates, there is also an external heating effect, an extension of the heating effect beyond the outside of the thermometer sheath because the generated joule heat must flow to some

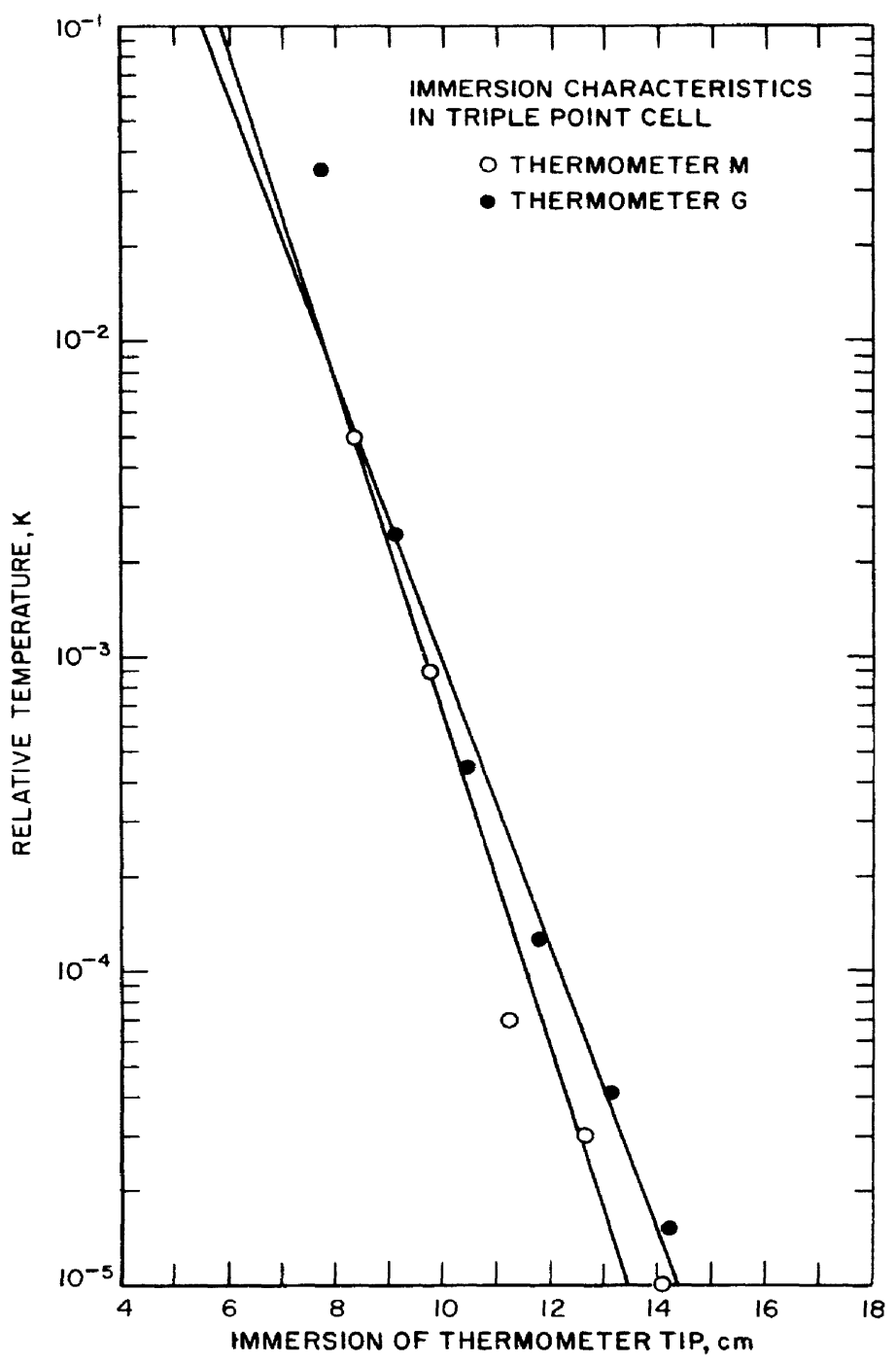

FIGURE 10. Immersion characteristics in the triple point of water cell of two long stem SPRT's with different sheath materials and internal construction.

The plot shows the relationship between the logarithm of relative temperature and the depth of thermometer immersion. Comparison with figure 9 shows that the immersion characteristics of the thermometers tend to be poorer in the triple-point cell than those in the ice bath. This change in the observed immersion characteristics is caused primarily by the higher resistance to radial heat flow in the triple-point cell.

external heat sink. The total heating effect, i.e., the combined internal and external heating effects, can easily be determined by calculating the resistance that would be measured at zero current; this can be performed either algebraically or graphically as shown in figure 13. When it is desirable to determine the internal heating effect only, the experimental conditions must be such that the external heating effect is negligible. This condition can be closely approximated by directly immersing the thermometer into an ice bath wherein the solid ice particles are in contact with the thermometer sheath, or into a metal (tin or zinc) freezing point apparatus in which the metal freezes directly on the thermometer. (The metal must be remelted before complete solidification or the thermometer will be crushed.) 


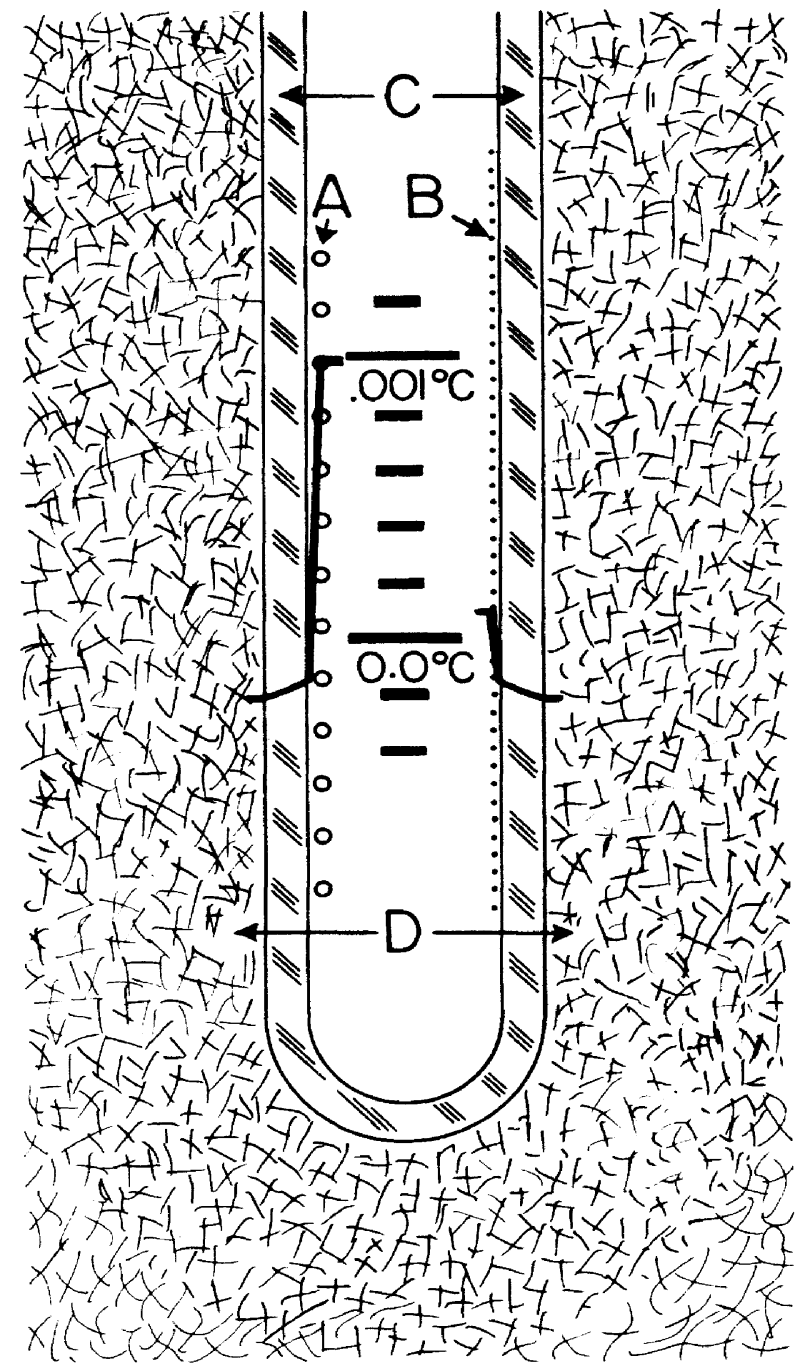

Figure 11. Platinum resistance thermometers at $33 \mathrm{~cm}$ immersion in an ice bath.

Temperature profile from the middle of thermometer coil out to the ice bath with $1 \mathrm{~mA}$ curren

A. Platinum coils of coiled filament thermometer; only coils of one side are indicated.

B. Platinum coils of single layer helix thermemeter: only turns of one side are indicated

C. Borosilicate glass thermometer envelope.

D. Finely divided ice and water.

The recommended measurement and calculation procedures are identical to those previously described for determining the total heating effect (see fig. 13). For the most precise work, all resistance measurements should be made at two currents and use made of the value of resistance calculated for zero current. Reiterating what was said earlier, an error due to the heating effect is introduced if the thermometer is not calibrated and used at the same current or is not in good thermal contact with its surroundings.

The external heating effect may be reduced by making the thermometer well a relatively close fit to the thermometer and placing a material of high thermal conductance in the annular space between the thermometer and the well. The material used to fill the space must not undergo an exothermic (or endothermic) reaction at the temperatures involved because such a reaction would additionally change the temperature of the thermometer from that of the surroundings. Examples of this difficulty that have been experienced in the NBS calibration laboratory are the slow decomposition of light mineral oil (at $122{ }^{\circ} \mathrm{C}$ ) and oxidation of a steel bushing (at $444{ }^{\circ} \mathrm{C}$ ). Difficulties associated with this type of reaction can be detected by comparing the derived values of resistance at zero current that have been obtained from measurements in which (i) the questionable material was used and (ii) the material was not present, or a better substitute was employed.

Another source of heat flux to and away from the platinum coil, and consequently a possible source of error, is radiation. If the sensor can "see" a surface that is appreciably hotter or colder, the power gained or lost by the resistor will result in its temperature being changed. In the triple-point of water measurements, radiation from lights in the room which is incident upon the top of the ice bath or triple point cell can easily produce an error of $0.0001 \mathrm{~K}$ (see sec. 7 on triple-point cell). The water-triple-point cell should, therefore, be immersed in an ice bath in which no extraneous radiation from sources above room temperature can reach the sensor of the SPRT. At a higher temperature $\left(630{ }^{\circ} \mathrm{C}\right)$ an error as large as $0.01{ }^{\circ} \mathrm{C}$ can occur if a clear fused silica thermometer sheath is employed because "light piping" takes place. In this process radiation is conducted towards ambient temperatures within the wall of the thermometer sheath, being confined there by total internal reflections. Any technique which would eliminate these reflections would of course, eliminate this source of error. The error can be substantially reduced by coating the exterior wall with graphite paint as is depicted in figure 14 or by roughening (by sand-blasting) the external wall. 


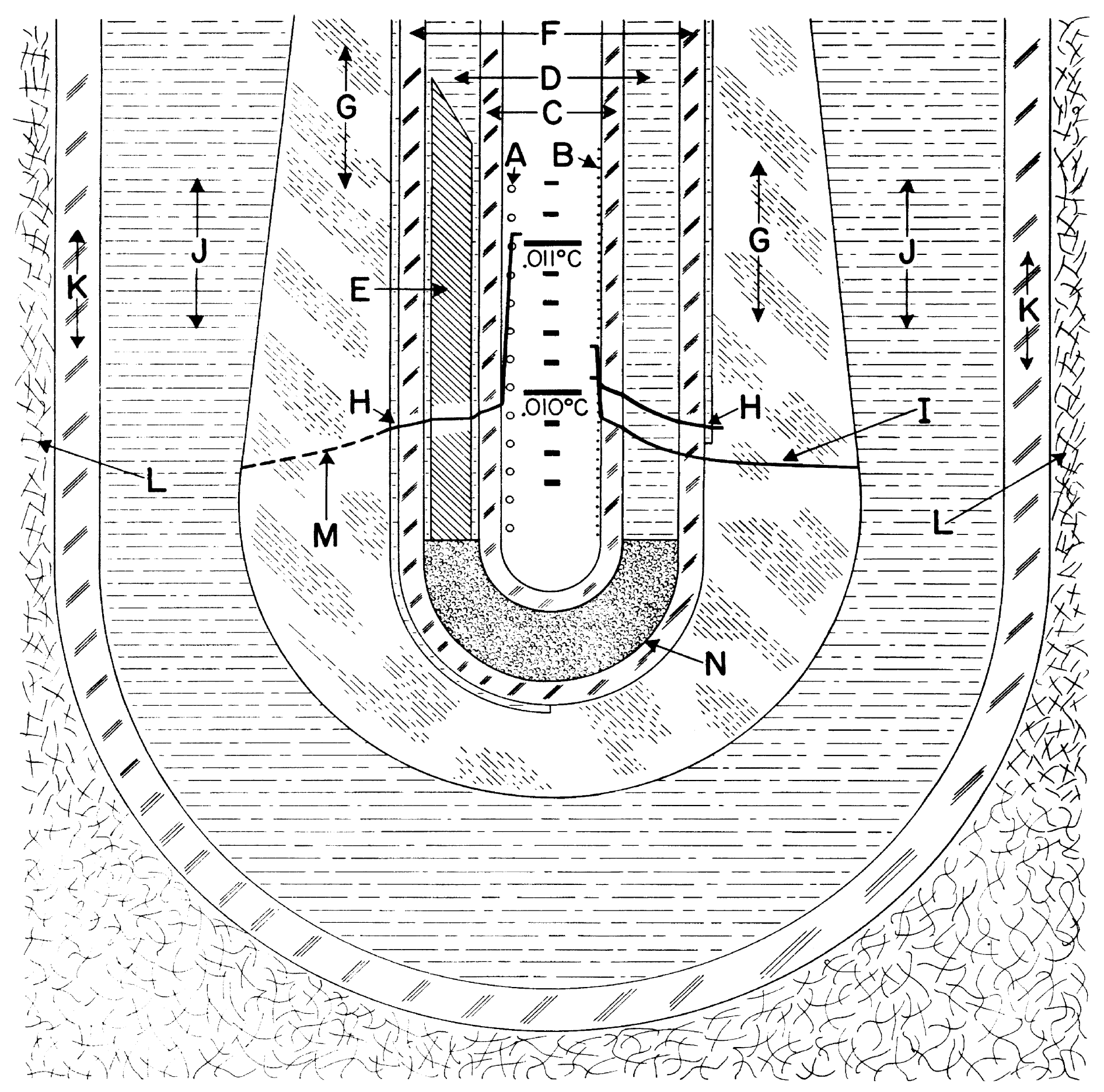

Figure 12. Platinum resistance thermometers at $33 \mathrm{~cm}$ immersion in a water triple point cell.

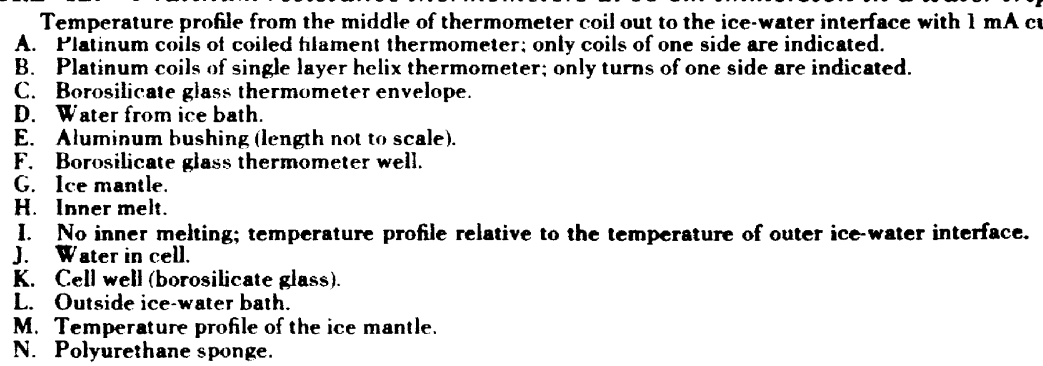




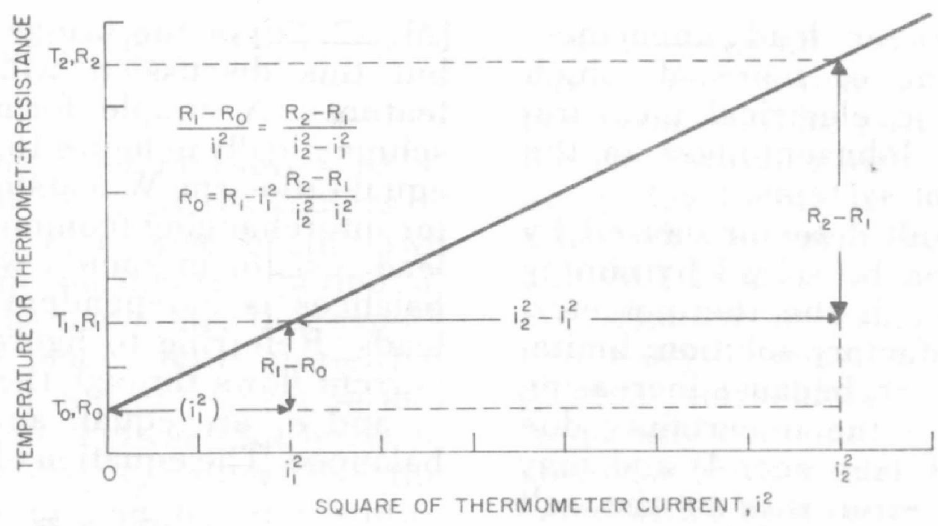

FIGURE 13. Plot of SPRT resistance (temperature) versus the square of the thermometer current.

The plot illustrates how the value of "zero current" thermometer resistance may be obtained graphically or algebraically from measurements at two currents.

$R_{0}=$ resistance of SPRT at "zero current."

$R_{1}$ and $R_{2}=$ resistances determined at currents $i_{1}$ and $i_{2}$, respectively,

FIGURE 14. Two methods for reducing radiation "piping" in fused silica thermometer sheaths. The sheath of the thermometer at the top was given a matte finish by "sand blasting." "Aqua dag" is shown painted on the sheath of the SPRT at the bottom.

\section{Resistance Measurements}

In this section, the salient features of instrumentation used to measure the resistance of SPRT's in the NBS calibration laboratory will be described so that the laboratory's general electrical measurement procedures may be understood. The discussion will neither give details on other instruments that are available for measuring electrical resistance nor special design features of particular instruments, nor a comparison of different measurement techniques. Some of the instruments will be mentioned by name and literature references will be cited to direct an interested reader to more detailed information.

The most suitable methods for and the limitations on achieving both accurate and reproducible resistance measurements depend on several factors. Johnson noise [31], which is inherent in any resistor (caused by the random movement of electrons within a conductor), is given by:

$$
e=7.43 \times 10^{-12} \sqrt{T R(\Delta f)},
$$

where $e$ is the thermal agitation voltage in volts, $\Delta f$ is the effective bandwidth in hertz, $R$ is the resistance of the conductor in ohms, and $T$ is the temperature of the resistor in kelvins. The constant $7.43 \times 10^{-12}$ comes from $2 \sqrt{k}$, where $k$ is the Boltzmann constant. However, this frequently mentioned limitation is seldom the predominant source of uncertainty in resistance measurements with SPRT's. For example, using a $25 \Omega$ thermometer at room temperature with an observation (averaging) time of 1 second, this noise is of the order of $0.6 \mathrm{nV}$-slightly less than the signal $(1.0 \mathrm{nV})$ that results from a $1-\mu \Omega$ unbalance when $1 \mathrm{~mA}$ is flowing through the thermometer. Spurious emf's, variations in contact resistance, mechanical or electrical disturbance of the detector system, 
and variations in thermometer lead unbalance, are sources of "noise," one or more of which contribute much more to the electrical measurement uncertainty than the Johnson noise in the vast majority of measurement systems.

The signal level at the null detector caused by the resistance unbalance can be raised by simply increasing the current through the thermometer. This may at times be a satisfactory solution; limitations are encountered, however, because increasing the current rapidly increases the uncertainty due to the self-heating of SPRT (see sec. 4) and may even introduce significant error due to the self heating of reference resistors in the bridge or potentiometer. The increased power dissipated in the thermometer may produce additional difficulties if the thermometer is used in a calorimeter system with a small heat capacity.

In addition to the uncertainty of null detection in a reasonable period of time, there is also the problem of referencing the unknown resistor to a single resistance standard. A large number of arrangements have been proposed and used for this purpose. They may be broadly classified as either bridge or potentiometer circuits that employ either alternating or direct currents with either resistors or inductive dividers to establish the ratio of the unknown resistor to a standard resistor. (A standard resistor is defined, hereafter, as a stable resistor or a combination of stable resistors of known value.)

\subsection{Mueller Bridge}

At the National Bureau of Standards the traditional instrument used with SPRT's is the Mueller bridge $[37,38]$. There have been several modifications

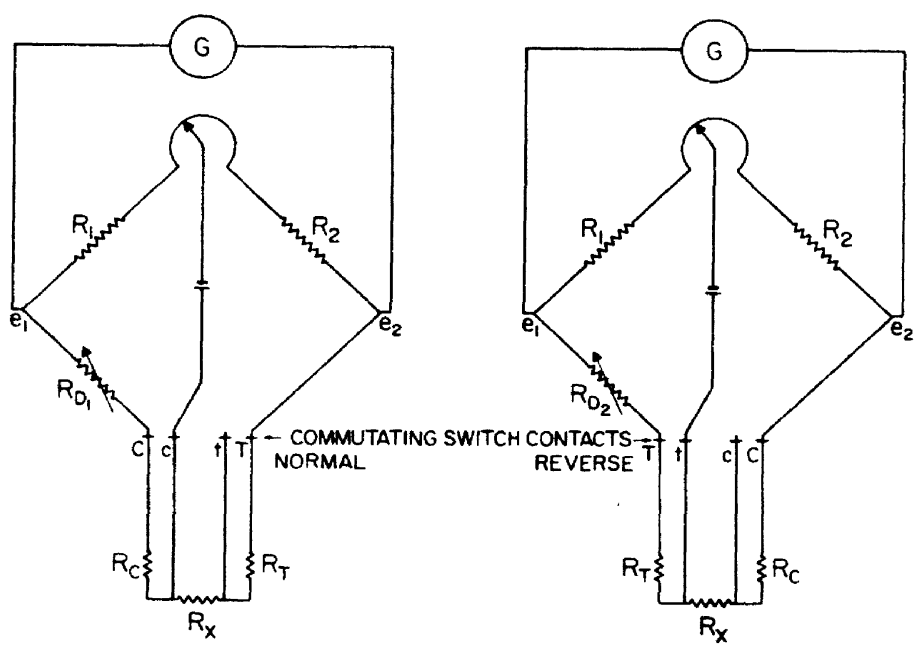

FigURE 15. Schematic of a Mueller bridge circuit in NORMAL and REVERSE thermometer connections.

$C$ is the null detector (galvanometer).

$\boldsymbol{R}_{1}$ and $\boldsymbol{R}_{2}$ represent the ratio arm resiators.

$\boldsymbol{R}_{\boldsymbol{D}}$ represents the adjustable resistor.

$\boldsymbol{R}_{r}$ and $\boldsymbol{R}_{\mathrm{T}}$ are the resistances of the "potential" leads of the SPRT.

$R_{x}$ is the resistance of the SPRT.
$[21,22,26]$ of the bridge since its first appearance, but this discussion will only cover its principal features. A simple form of the bridge is shown schematically in figure 15 . It is basically the classical equal-ratio-arm Wheatstone bridge with provisions for interchanging (commutating) the leads of a fourlead resistor in such a way that the average of two balances is independent of the resistances of the leads. Referring to figure 15 (NORMAL), when no current flows through the null detector, the voltages $e_{1}$ and $e_{2}$ are equal, and the bridge is said to be balanced. The equation of balance is:

$$
R_{D_{1}}+R_{C}=R_{X}+R_{T}
$$

where $R_{D_{1}}$ is the resistance of the variable decade balancing resistor,

$R_{C}$ is the resistance of a lead from the bridge to the thermometer sensing element,

$R_{X}$ is the resistance of the thermometer sensing element, and

$\boldsymbol{R}_{T}$ is a lead resistance similar to $\boldsymbol{R}_{C}$.

After commutating the leads (marked $C, T, c$, and $t$ ) to the positions shown in figure 15 (REVERSE) the equation of balance is

$$
R_{D_{2}}+R_{T}=R_{X}+R_{C},
$$

where

$R_{D_{2}}$ is the resistance of the adjustable-decade balancing resistor that is required for the second balance of the bridge.

The addition of eqs (5.2) and (5.3) yields the value of $R_{X}$ in terms of $R_{D_{1}}$ and $R_{D_{2}}$ :

$$
R_{D_{1}}+R_{D_{2}}=2 R_{X}
$$

or

$$
R_{X}=\frac{\mathbf{R}_{D_{1}}+D_{D_{2}}}{2}
$$

An assumption made in the above equations is that $\boldsymbol{R}_{T}$ and $\boldsymbol{R}_{C}$ are constant during the time required for making the two balances.

Actually $\boldsymbol{R}_{T}$ and $\boldsymbol{R}_{C}$ need not be constant. If eqs (5.2) and (5.3) are rewritten as:

$$
R_{D_{1}}+R_{C_{1}}=R_{X}+R_{T_{1}}
$$

and

$$
\boldsymbol{R}_{D_{2}}+\boldsymbol{R}_{T_{2}}=\boldsymbol{R}_{X}+\boldsymbol{R}_{C_{2}},
$$

and then added, there results

$$
\boldsymbol{R}_{D_{1}}+\boldsymbol{R}_{D_{2}}+\left(\boldsymbol{R}_{C_{1}}-\boldsymbol{R}_{T_{1}}\right)-\left(\boldsymbol{R}_{C_{2}}-\boldsymbol{R}_{T_{2}}\right)=2 \boldsymbol{R}_{X}
$$

or 
$R_{X}=\frac{R_{D_{1}}+R_{D_{2}}}{2}+\frac{\left(R_{C_{1}}-R_{T_{1}}\right)-\left(R_{C_{2}}-R_{T_{2}}\right)}{2}$.

Thus, a sufficient condition for the measured resistance to be independent of the lead resistances is that the difference in the resistance of the two potential leads is constant during the period of observation. Equation (5.9) demonstrates that experimental emphasis could be placed on insuring that the leads be of equal length and cross section and that the temperature gradients between the leads be constant or only changing slowly, rather than the more difficult option of maintaining the temperature of the leads constant.

Successful operation of the Mueller bridge is dependent upon the reproducibility and self consistency (linearity) of the adjustable resistor indicated in figure 15 as $R_{D}$. The methods of accomplishing this include thermostating the resistors and employing special circuitry. (See fig. 1 of Appendix H. A procedure for calibrating the Mueller bridge is also described in Appendix H.) The circuitry has been designed to reduce the uncertainties that are associated with the variations in the contact resistances of the "dry" switches. For the $1-\Omega$ and $0.1-\Omega$ step decade resistors, the switch contact resistances are placed in series with the bridge ratio arms $\left(R_{1}\right.$ and $\left.R_{2}\right)$, which are usually from 500 to $3000 \Omega$, so that the effect of the possible variations in the contact resistances (about $0.0005 \Omega$ ) can usually be neglected. For the measurement of the higher thermometer resistances this arrangement introduces uncertainties which may be significant. For a $25-\Omega$ SPRT the uncertainty of $0.0005 \Omega$ in $3000 \Omega$ or 1 part in $6 \times 10^{6}$ in the ratio arm corresponds to only $1 \mu \Omega$ or $0.01 \mathrm{mK}$ when measuring $6 \Omega$ (near $-183{ }^{\circ} \mathrm{C}$ ) but increases to 10 $\mu \Omega$ or $0.1 \mathrm{mK}$ when measuring $60 \Omega$ (near $350^{\circ} \mathrm{C}$ ). The decades with steps of $0.01 \Omega$ or less are the Waidner-Wolff shunted decades [38] which reduce the effect of contact resistance in the switch by a factor of 250 or more. The switches for the $10-\Omega$ step decade and the commutator are directly in series with the resistors in the adjustable arm of the bridge and the thermometer resistor; they have, therefore, mercury-wetted contacts. The mercurywetted switches in the Mueller bridges employed at the National Bureau of Standards have an uncertainty of less than $2 \mu \Omega$ when well maintained. All of the mercury contacts are normally cleaned every day before use. The mercury is removed by vacuum using a small polyethylene tip at the end of a vacuum line with a mercury trap. Fresh mercury is placed on each amalgamated contact. If the entire surface of the contact is not wetted by the new mercury, the surface is scrubbed (without removing the mercury) with the flat end of a solid copper rod about $1 / 4$ inch in diameter until the entire surface becomes wetted. The mercury is again removed and replaced with clean mercury. The switch is then reassembled and operated several times after which it is reopened to remove any mercury which has splashed onto the surrounding surfaces. The switch is then finally assembled for use. Switches with sliding contacts are exercised every day before use by revolving them 10 or 20 times; this is particularly important for the $1,0.1$, and $0.01 \Omega$ decade switches. The sliding switches are cleaned occasionally with a lint-free cloth, either dry or moistened with benzene or "varsol". (Carbon tetrachloride is not recommended as it frequently contains impurities which will result in corrosion.) After cleaning, the contacts are lubricated with a light coating of pure petrolatum.

\subsubsection{Bridge Ratio Arms}

If the two ratio resistors, $R_{1}$ and $R_{2}$ in figure 15 , change so as to become unequal, this may be compensated by adjusting the "tap" on the slidewire resistor joining $R_{1}$ and $R_{2}$. Because this tap is in the battery arm of the bridge, the variations of its contact resistance are unimportant to the determination of thermometer resistances. The ratio arm resistors are adjusted to be equal by varying this tap on the slide wire between $R_{1}$ and $R_{2}$ until the interchanging of the ratio arms does not change the bridge balance. The accuracy of the ratio is limited by the uniformity of the resistance of the copper leads and switch contacts which are in series with $R_{1}$ and $R_{2}$ (those connecting the $1-\Omega$ and $0.1-\Omega$ decades) and, of course, by the sensitivity of the null detector.

Some versions of the bridge have incorporated into the commutator additional switch contacts which reverse the ratio arm resistors simultaneously with each commutation of the thermometer leads; the switch contacts of the $1-\Omega$ and $0.1-\Omega$ decades and their leads to the commutator switch are, however, not reversed. The effect of their variations must still be considered in the determination of the bridge resistance. Referring again to figure 15, but now with the ratio arms $R_{1}$ and $R_{2}$ interchanged in the reverse bridge connection, the equations of bridge balance with the normal and reverse connections are, respectively:

and

$$
\frac{R_{D_{1}}+R_{C}}{R_{T}+R_{X}}=\frac{R_{1}}{R_{2}}=\frac{1+\epsilon}{1}
$$

$$
\frac{R_{D_{2}}+R_{T}}{R_{C}+R_{X}}=\frac{R_{2}}{R_{1}}=\frac{1}{1+\epsilon},
$$

where $\epsilon=\left(R_{1}-R_{2}\right) / R_{2}$. After combining eqs (5.10) and (5.11) and eliminating $R_{T}$ and $R_{C}$,

$$
R_{X}=\frac{R_{D_{1}}+R_{D_{2}}(1+\epsilon)}{2+\epsilon} .
$$

By adding and subtracting $\left(R_{D_{1}}+R_{D_{2}}\right) / 2$ from the right-hand side of eq (5.12) and combining, the 
relation

$$
R_{x}=\frac{\left(R_{D_{1}}+R_{D_{2}}\right)}{2}+\frac{\left(R_{D_{1}}-R_{D_{2}}\right) \epsilon}{2(2+\epsilon)}
$$

is obtained. Equation (5.13) shows that if the bridge is operated to yield $R_{D_{1}}=R_{D_{2}}$, then $R_{X}=R_{D_{1}}=R_{D_{2}}$, without regard to the lack of equality of the ratioarm resistors. In practice, because the thermometer leads $(T$ and $C$ ) are usually made nearly equal, $R_{D_{1}}$ and $R_{D_{2}}$ will be only slightly different, typically much less than $0.002 \Omega$. Also, the equality of the ratio-arm resistors can be adjusted to better than $1 \mathrm{ppm}$ with ease. Thus, the second term on the right of eq (5.13) is completely negligible in normally conducted Mueller bridge measurements which utilize simultaneous commutation of the SPRT leads and the ratio-arm resistors.

\subsubsection{Bridge Current Reversal}

The indicated balance of any $d-c$ bridge is dependent upon the $i R$ voltages across the elements of the circuit and the spurious emf's. The equations of bridge balance involve only resistance values; therefore, the effects of spurious emf's must be eliminated. This could be done by first observing the "indication" of the null detector with no bridge current, then balancing the bridge with current to the same indication. The indication of the null detector with no current includes the effect of any spurious emf's; when the bridge is balanced to the same indication with current in the bridge, the $i R$ voltages are balanced if the effect of the spurious emf's remains unchanged. However, one should recognize that a change in the magnitude of the thermometer current will change the thermometer temperature (due to self heating) and that enough time must elapse before reading to allow the thermometer to attain a steady thermal state. During this time the effect of spurious emf's may change significantly. This problem can be simply surmounted by reversing the battery current with a snap action toggle switch, so that an essentially continuous heating power is retained in the thermometer (hence, a uniform self heating effect). In the process, the galvanometer or null detector sensitivity is effectively doubled. The rate at which the current reversals must be made is dependent on the rate of change of the spurious emf's.

\subsection{A-C Bridge}

If the current is reversed sufficiently rapidly, it is usually said that the bridge is an "a-c" bridge. Bridges operating at 400 hertz have been built at the NBS based on a design by Cutkosky [19]. These bridges were designed for use with SPRT's and include special provisions for use with thermometers having values of $R(0)$ as low as $0.025 \Omega$. The bridge utilizes an inductive ratio divider that eliminates the necessity of calibrating the bridge because the initial uncertainty of the divider is about 2 parts in $10^{8}$ and appears to be stable. Additionally, the bridge requires only one manual resistance balance, the phase angle balance being automatic, and incorporates a built-in phase-sensitive null detector with which $1 \mu \Omega$ in $25 \Omega$ can be easily resolved. Small deviations from balance can be recorded continuously, the accuracy of recording these deviations being limited primarily by the resolution and linearity of the recorder. A small (usually less than $10 \mu \Omega$ ) error may be introduced in measuring a $25 \Omega$-SPRT unless coaxial leads are used between the bridge and the thermometer head. (The heads of SPRT's have been modified to contain two BNC coaxial connectors. The two leads from one end of the SPRT coil were connected to the center "female" contracts of the BNC receptacles and the two leads from the other end of the SPRT were connected to the outer shells or the shield contacts.) For precision measurements the length of the pair of coaxial leads should not be greater than 15 meters to limit the dielectric losses of the shunt capacitance. Preliminary measurements on $25-\Omega$ SPRT's indicate that, if the leads do not affect the measurements, the accuracy of the measured value in ohms of a thermometer element is limited by the accuracy to which the reference standard resistor is known. However, in the accurate determination of the resistance ratio, $R(t) / R(0)$, the stability, rather than the accuracy, of the reference standard is the important requirement. Further work is in progress at NBS to determine the dc-ac transfer characteristics of SPRT's.

\subsection{Potentiometric Methods}

The Mueller bridge and the Smith bridge methods $[6,7,27]$ are suitable for a four-terminal determination of thermometer resistance if the lead resistances are relatively stable. When the lead resistances are variable, e.g., in measurements at low temperatures where the cryostat temperature is varying and the thermometer resistance is small, potentiometric methods become better suited to the measurements.

The potentiometric method of resistance measurement depends in principle upon the determination of the ratio of $i R$ voltages developed across the SPRT and across a resistor of known resistance that is connected in series. The ratio is determined by comparisons with $i R$ voltages that are developed in a separate resistance network and usually a separate current supply.

One of the major drawbacks of the potentiometric method has been the requirement of exceptionally high stability of current in both the potentiometer and in the SPRT circuits during the measurement period. However, the recent availability of highly stable current supplies and their continued improvement have made the potentiometric methods more popular. 
Three circuits for the more commonly used potentiometric methods are illustrated in figure 16 . To eliminate the effect of spurious emf's in the measurements employing circuits (a) and (b) the currents $i_{1}$ and $i_{2}$ are reversed. Four balances are necessary in each method, two with the current in one direction and two with the current reversed. Four balances are also necessary employing circuit (c) by reversal of the current $i_{1}$ and at the connection between the voltages to be com- pared. The readings for the normal and reverse connections are averaged. By using high quality reversing switches along with current sources of high resistance (to make the relative effect of small changes in resistance associated with the switching negligible compared to the total resistance of the circuit), the current in the circuit can be made more stable and the switch in series with the detector need not be disturbed during the current reversal process. Nevertheless, a shunt is needed across the

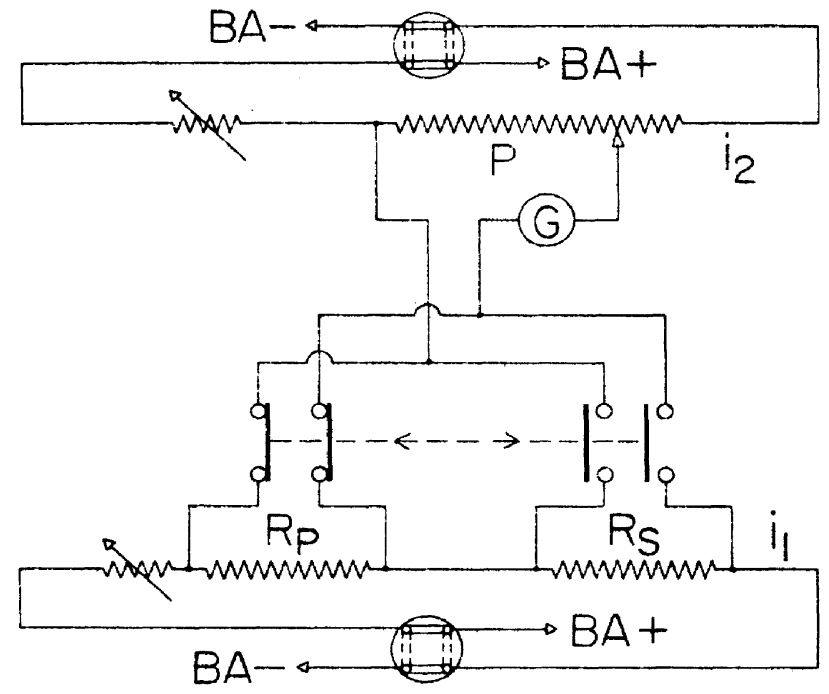

(a)

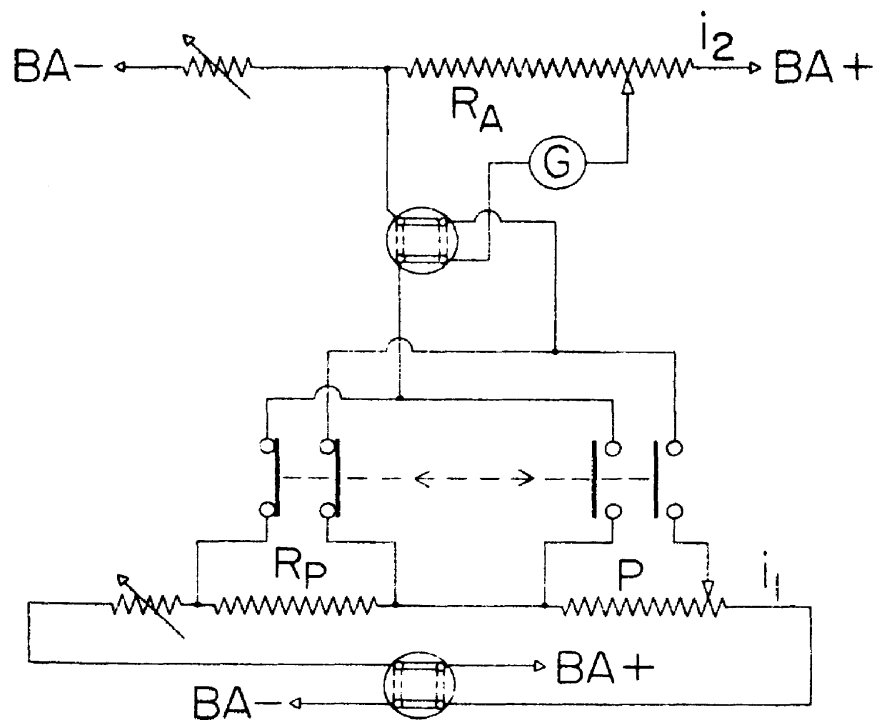

(c)

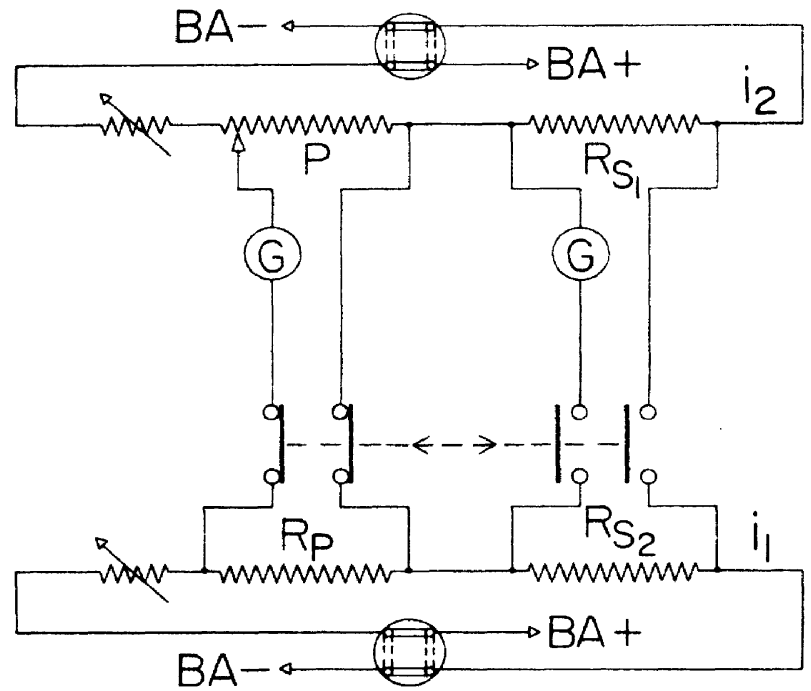

(b)

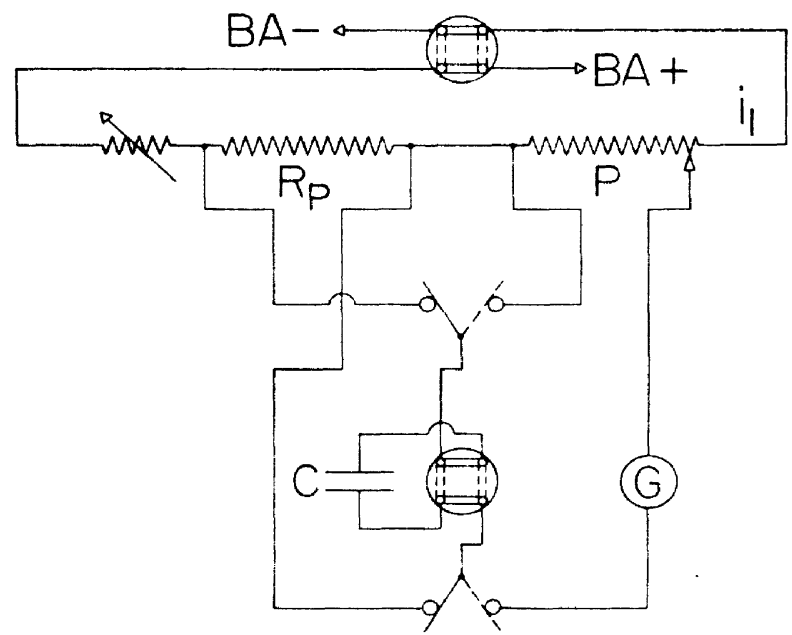

(d)

Figure 16. Potentiometric circuits used to determine resistance values.

$R_{p}$, the resistance to be determined.

$\boldsymbol{R}_{n}$, standard resistor of precisely known value.

$\boldsymbol{P}$, potentiometer or precisely known adjustable resistor.

$P$, potentiometer or preci

$\boldsymbol{C}$, stable capacitor of low dielectric absorption, low absorption time constant, and high leakage resistance (polystyrene).

$G$, null detector

$i_{1}$, independent stable current in the unknown resistance circuit.

$i_{2}$, independent stable current in the potentiometer or in the volt age transfer instrument circuit. 
detector to protect it because of asynchronous contact operation during the reversal. The discussion of the potentiometric methods to follow assumes that the required balances are made with current reversal.

Using circuit (a) of figure 16, the potentiometer $P$ is successively balanced against the voltages $i_{1} R_{p}$ and $i_{1} R_{s}$ in terms of $i_{2} P_{p}$ and $i_{2} P_{s}$, respectively, where $R_{p}$ and $R_{s}$ are the resistances of SPRT and standard resistor, respectively, and $P_{p}$ and $P_{s}$ are in the potentiometer resistance units. $\left(P_{p}\right.$ indicates the potentiometer resistance when its $i R$ voltage is balanced against the SPRT; $P_{s}$ indicates the potentiometer resistance when balanced against a standard resistor.) Thus, with $i_{1} R_{p}=i_{2} P_{p}$ and $i_{1} R_{s}=i_{2} P_{s}$, the SPRT resistance is given by

$$
\boldsymbol{R}_{p}=\frac{\boldsymbol{P}_{p}}{\boldsymbol{P}_{s}} \boldsymbol{R}_{\boldsymbol{s}}
$$

There is a wide variety of potentiometers that employ essentially the circuit shown in 16(a). The potentiometer $i R$ voltages in some designs $[43,54$, $55,56]$ are developed with different currents in certain decades; in others, the $i R$ voltages are adjusted by varying, by means of the potentiometer switches, the current through a fixed resistor. These designs keep the resistance of the detector circuit constant. In the "double potentiometer," which is designed to make two consecutive voltage balances more conveniently, there are duplicate sets of switches but only a single resistance network to develop $i R$ voltages. A requirement of the potentiometer, whatever the design, is that the "resistance units" or the " $i R$ voltage units" that are developed in the instrument for voltage balance be linear.

Two standard resistors are used in circuit (b). Current $i_{2}$ is adjusted until $i_{2} R_{s_{1}}=i_{1} R_{s_{2}}$; then $P$ is adjusted until $i_{2} P_{p}=i_{1} R_{p}$. The SPRT resistance is given by:

$$
R_{p}=\frac{R_{s_{2}}}{R_{s_{1}}} P_{p}
$$

In circuit (c), either current $i_{2}$ or resistance $R_{A}$ is adjusted until $i_{2} R_{A}=i_{1} R_{p}$; then $P$ is adjusted until $i_{2} R_{A}=i_{1} P_{p}$. The thermometer resistance is given directly by $R_{p}=P_{p}$.

The "isolating potential comparator" method described by Dauphinee [19] is an adaptation of circuit (c) where the voltage $i_{1} R_{p}$ is set up as $i_{2} R_{A}$ and measured as $i_{1} P_{p}$. The method is shown in circuit (d). The voltage $i_{1} R_{p}$ appears across a high quality capacitor $C$ and is compared with the voltage $i_{1} P_{p}, P$ being adjusted until $i_{1} R_{p}=i_{1} P_{p}$. The breakbefore-make, double-pole chopper switches the capacitor alternately across $R_{p}$ and then across $P$ between 20 to 80 times a second. Extraneous voltages are canceled by reversing the current and the capacitor connections and averaging the second reading with the first.
The linearity of the potentiometer can be calibrated by comparing successive steps of one decade against the total $(X)$ of the next lower decade. See Appendix I for a method of calibrating potentiometers.

\section{Calculation of Temperatures from the Calibration Data and Observed Resistances}

The SPRT is the standard interpolation instrument between the defining fixed points in the range $13.81 \mathrm{~K}$ to $630.74{ }^{\circ} \mathrm{C}$. (See table 1 and Appendix A.) The "constants" of the interpolation formulae that relate the resistance of a particular SPRT to the value of its temperature on the IPTS-68 are obtained by resistance measurements at the defining fixed points. At NBS the "long stem" SPRT's are usually calibrated for application above $90 \mathrm{~K}$; the capsuletype SPRT's are calibrated for use between $13 \mathrm{~K}$ and $250^{\circ} \mathrm{C}$ or occasionally up to $400^{\circ} \mathrm{C}$. The equipment and procedures employed at NBS to achieve the fixed points are described in the next section. The resistance measuring instruments employed at NBS and other instruments that can be used for resistance measurements of SPRT have been described earlier in section 5 . This section deals with the methods in use at NBS to obtain the constants of the interpolation formulae from the calibration measurements of an SPRT at the fixed points. Methods of calculating temperatures from the observed resistances, when the constants of the interpolation formulae are known are also described. At NBS all evaluations of equations and calculations pertaining to the SPRT are performed on a highspeed electronic digital computer (UNIVAC 1108).

\subsection{Temperatures from 0 to $630.74{ }^{\circ} \mathrm{C}$}

From 0 to $630.74{ }^{\circ} \mathrm{C}$ the temperatures on the IPTS-68 are defined by

$$
t_{68}=t^{\prime}+M\left(t^{\prime}\right)
$$

where

$$
\begin{array}{r}
M\left(t^{\prime}\right)=0.045\left(\frac{t^{\prime}}{100}\right)\left(\frac{t^{\prime}}{100}-1\right) \\
\left(\frac{t^{\prime}}{419.58}-1\right)\left(\frac{t^{\prime}}{630.74}-1\right)
\end{array}
$$

and

$t^{\prime}=\frac{1}{\alpha}\left(\frac{R\left(t^{\prime}\right)}{R(0)}-1\right)+\delta\left(\frac{t^{\prime}}{100}\right)\left(\frac{t^{\prime}}{100}-1\right)$.

In the official English version of the text of the scale (see Appendix A) the unit ${ }^{\circ} \mathrm{C}$ appears in 
the defining equations, e.g.,

$$
\begin{aligned}
t_{68}=t^{\prime}+0.045 & \left(\frac{t^{\prime}}{100^{\circ} \mathrm{C}}\right)\left(\frac{t^{\prime}}{100^{\circ} \mathrm{C}}-1\right) \\
& \left(\frac{t^{\prime}}{419.58{ }^{\circ} \mathrm{C}}-1\right)\left(\frac{t^{\prime}}{630.74^{\circ} \mathrm{C}}-1\right){ }^{\circ} \mathrm{C} .
\end{aligned}
$$

In this discussion the equations will be simplified by omitting the units. Similarly $R\left(t^{\circ} \mathrm{C}\right)$ and $R\left(0^{\circ} \mathrm{C}\right)$ will be simplified to $R(t)$ and $R(0)$. In addition, $t_{68}$ will, henceforth, be abbreviated to $t$. The constants $R(0), \alpha$, and $\delta$ are determined from calibration resistance measurements of the SPRT at the triple point of water (TP), the steam point or the tin point, and the zinc point. (The tin point is now employed at NBS.) The constants may be more conveniently obtained from (6.1) and from the relation, equivalent to equation (6.3), given by:

$$
W\left(t^{\prime}\right)=R\left(t^{\prime}\right) / R(0)=1+A t^{\prime}+B t^{\prime 2}
$$

where $R\left(t^{\prime}\right)$ is the observed resistance at the temperature $t^{\prime}$ and $R(0)$ is the resistance at $0{ }^{\circ} \mathrm{C}$. The constants $A$ and $B$ are related to $\alpha$ and $\delta$ by

$$
\begin{aligned}
& A=\alpha(1+\delta / 100), \\
& B=-10^{-4} \alpha \delta
\end{aligned}
$$

also,

$$
\begin{aligned}
& \alpha=A+100 B, \\
& \delta=-10^{4} B /(A+100 B) .
\end{aligned}
$$

Equations (6.3) and (6.5), appear to be of the same form as the earlier formulations of the International Temperature Scale $[15,46,48]$. But, the value of temperature, $t$, on the IPTS-68 is not equal to $t^{\prime}$, and the value of $t^{\prime}$ is not the value of temperature on the IPTS -48 because the definitions of the two scales are different (see Appendix B).

The value $t^{\prime}$, obtained from (6.3) or (6.5), may be considered to be a first approximation to $t$, the value of temperature on the IPTS-68. Equation (6.2) gives the adjustment to be made to the value $t^{\prime}$ to yield $t$. This adjustment was included in the definition of the IPTS-68 with the intention of bringing the scale into closer agreement with the thermodynamic scale. Therefore, at any given hotness (except certain fixed points) the values of $t^{\prime}$ and $t$ are different, but they both represent the same hotness; accordingly, the resistances $R\left(t^{\prime}\right)$ and $R(t)$ are equal. The observed SPRT resistance will, hereafter, be indicated by $R(t)$.

At the NBS, the SPRT that is received for calibration is first annealed between 470 and $480{ }^{\circ} \mathrm{C}$ for about four hours and then allowed to cool in air at the ambient conditions. The calibration measure- ments at the fixed points are made in the following sequence: TP, zinc point, TP, tin point, and TP. If any observed resistance is questioned, the measurement is repeated. Any additional tin or zincpoint measurements will usually be bracketed before and after by TP measurements in order to check any change in the TP resistance that may occur. Whenever the TP resistance of a SPRT changes by more than $75 \mu \Omega$ (depending upon the design) in the course of calibration the complete sequence of calibration, including the annealing, is repeated.

A measuring current of $1 \mathrm{~mA}$ through the resistor of the thermometer is normally used. The sequence of measurements with the Mueller bridge at each of these fixed points is, unless additional measurements are found necessary, NRRN, where $N=$ normal and $R=$ reverse connections of the bridge. The readings are corrected for the "bridge calibration" and "bridge zero" and averaged (see sec. 5 on Mueller bridge resistance measurements).

For the tin and zinc-point calibration measurements, the "equilibrium temperatures" at the external surface of the SPRT near the resistance coil are computed and assigned to the observed resistance values. The experimental equilibrium temperature value is slightly different from the IPTS-68 value of the fixed-point temperature; the value of the equilibrium temperature at the outer surface of the thermometer sheath is obtained by correcting the IPTS-68 fixed-point temperature value for the hydrostatic head of the liquid metal column (also, for any significant deviation from 1 atm gas pressure over the fixed-point cell) and for the external heating effect of the SPRT in the particular cell. In the case of the measurements in a triple point of water cell, the assigned value of the temperature is adjusted for the temperature effect of the hydrostatic head of the water column and external heating effect of the SPRT.

The value of $R(T P)$ that is measured after the $R($ tin) measurement is employed to obtain $W($ tin $)$; in a similar manner, the value of $R(T P)$ measured after the $R$ (zinc) measurement is employed to obtain $W$ (zinc). The value of $R(0)$ is calculated from $R$ (TP) according to eq (6.5) by assigning $B=0$ for the small temperature interval of the evaluation. The value of $A$ employed is $3.98485 \times 10^{-3}{ }^{\circ} \mathrm{C}^{-1}$, which is an average value for SPRT's calibrated at NBS in the past few years. The uncertainty in the adjustment of the value from $R(T P)$ to $R(0)$ in this manner is about $\pm 1 \times 10^{-8} R(0)$ or $\pm 0.25 \mu \Omega$ for a $25 \Omega$ SPRT and is, therefore, negligible. The thermometer constants $A$ and $B$ are obtained by simultaneous solution of eq (6.5) from the values of $W($ tin), $t^{\prime}\left(\right.$ tin), and $W\left(\right.$ zinc), $t^{\prime}$ (zinc). The constants $\alpha$ and $\delta$ are obtained from $A$ and $B$ according to eqs (6.8) and (6.9). The printed results of the computer analysis of calibration data include, in addition to tables of $W(t)$ or $R(t)$ versus $t$, the observed resistances (adjusted for bridge calibration and bridge 
zero) at the three fixed points, and the values of $R(0), \alpha, \delta, A$, and $B$.

Several possibilities are available to the user for computing temperatures and for checking the calibration of his SPRT. First, in eq (6.3) $R(0), \alpha$, $\delta$ may be taken as invariant and $t^{\prime}$ calculated from the measured $R(t)$. Second, in eq (6.3) only $\alpha$, and $\delta$ may be taken as invariant, requiring then the determination of $R(t) / R(0)$. The first method is equivalent to using a table of $R$ versus $t$; the second method is equivalent to using a table of $R(t) / R(0)$ versus $t$. Equation (6.3) may be rewritten as

$t^{\prime}=100 \frac{R(t)-R(0)}{R(100)-R(0)}+\delta\left(\frac{t^{\prime}}{100}\right)\left(\frac{t^{\prime}}{100}-1\right)$.

In eq (6.10), as a third method, $R(100)-R(0)$, $\alpha$ and $\delta$ may be taken as constant, requiring the determination of $R(t)-R(0)$ to calculate $t^{\prime}$. The fourth possibility seen with equation $(6.10)$ is to take only $\delta$ as constant which requires the determination of $\frac{R(t)-R(0)}{R(100)-R(0)}$. The ultimate extension of this procedure of reducing the SPRT constants is not to assume that anything has remained constant and recalibrate the thermometer every time it is used. In 80 years of platinum thermometry each of the above assumptions or its equivalent has been made by.investigators.

From the standpoint of accuracy the worst of these assumptions is the first, which requires only the measurement of $R(t)$. This choice may be dictated, however, by convenience or economy. If values of $R(0)$ can be obtained, the choice is not as clear between using $R(t) / R(0)$ or using $R(t)-R(0)$ to calculate the value of temperature. Experience with a particular thermometer may help one make the choice. Lacking this, one may be guided by the experience of the NBS and the National Research Council of Canada which indicates that below $500{ }^{\circ} \mathrm{C}$. the second assumption, namely, that $\alpha$ and $\delta$ are constant and using the value of $R(t) / R(0)$ to calculate a value of temperature, is the most satisfactory. This assumption has the distinctive practical advantage of being dimensionless (i.e., being independent of the size of the resistance unit). If request is made at the time the SPRT is submitted to NBS for calibration, tables based on any one of the assumptions discussed in this paragraph, in a form suitable for linear interpolation, can be provided from the calibration data.

When many observations of $R(t)$, such as in specific-heat measurements, are to be converted to $t$ and a high speed electronic computer is available, eqs $(6.1),(6.2)$, and $(6.3)$ or $(6.10)$ may be more convenient than tables. Iteration methods are best employed in the solution of either eq (6.3) or (6.10). For example, an approximate $t^{\prime}$ can first be obtained neglecting the term containing $\delta$. Improved approximations are obtained by substituting successive solutions into the complete equation, stopping when $t^{\prime}$ does not change more than desired limits. If it is desired to generate a table of values of $W(t)$ versus values of $t$, entries for integral values of $t$ at one-degree intervals seem quite useful. The error contributed by using simple linear interpolation within the one-degree intervals is less than $10^{-4}{ }^{\circ} \mathrm{C}$ in the range from 0 to $630^{\circ} \mathrm{C}$. The calculation of the table is most easily performed employing eqs (6.1) and (6.5). The only difficulty is that (6.5) gives $W$ as a function of $t^{\prime}$ rather that $t$, hence if $W$ is to be tabulated as a function of $t$ the value of $t^{\prime}$ corresponding to the value of $t$ must be first obtained and used in (6.5). By rearranging (6.1),

$$
t^{\prime}=\boldsymbol{t}-\boldsymbol{M}\left(\boldsymbol{t}^{\prime}\right)
$$

Below $472^{\circ} \mathrm{C}$ the error introduced by replacing $(6.11)$ with $t^{\prime}=t-M(t)$ is less than $1.2 \times 10^{-4}{ }^{\circ} \mathrm{C}$. The error in the value of $t^{\prime}$ to be used may be reduced below $10^{-5}{ }^{\circ} \mathrm{C}$ by a single iteration of eq (6.11). A very efficient equation for calculating the $n$th entry for tables with intervals of less than 10 degrees is

$$
t_{n}^{\prime}=t_{n}-M\left(t_{n}-M\left(t_{n}-M\left(t_{n-1}^{\prime}\right)\right),\right.
$$

Equation (6.12) does not require iteration and it may be shown that it introduces an error of only $\left[d M\left(t^{\prime}\right) / d t^{\prime}\right]^{2}$ times the tabular interval.

\subsection{Temperatures Below $0^{\circ} \mathrm{C}$}

From $0{ }^{\circ} \mathrm{C}$ down to $-259.34{ }^{\circ} \mathrm{C}$, the temperatures on the IPTS -68 are defined by

$$
W(t)=W^{*}(t)+\Delta W(t),
$$

where $W(t)=R(t) / R(0)$ is the ratio of the observed resistance of the SPRT at temperature $t$ to that at $0{ }^{\circ} \mathrm{C}$ and $W^{*}(t)$ is a reference value of the resistance ratio given by the function

$$
t=\sum_{i=1}^{20} A_{i}\left(\ln W^{*}(t)\right)^{i} .
$$

In this monograph the symbol $W^{*}(t)$ will replace the symbol $W_{\text {CCT-68 }}\left(T_{68}\right)$ used in the official text of the IPTS-68 to represent the reference (standard) values of $W$. The values of $W^{*}(t)$ represent a fictitious but not "ideal" platinum resistance thermometer. The constants $A_{i}$ of the reference function are listed in Appendix A. The deviations, $\Delta W(t)$ of eq (6.13), are expressed by simple polynomial equations of specified form; the constants of these equations are determined from measurements of $W(t)$ at the appropriate defining fixed points and the corresponding values of $W^{*}(t)$. The intermediate temperatures are determined from the corresponding measured values of $W(t)$, the appropriate polynomial equation for $\Delta W(t)$, and eqs $(6.13)$ and 
(6.14). (See Appendix A which lists the four temperature ranges, the associated polynominal equations, and the fixed points defined by the IPTS -68 in the range -259.34 to $0^{\circ} \mathrm{C}$.) The text of the scale, given in Appendix A, states the deviation equations for use below the oxygen point as functions of $T$ (in kelvins). This form of these equations will also be used in this monograph because of their greater simplicity. It follows that eq (6.13) becomes

$$
W(T)=W^{*}(T)+\Delta W(T),
$$

and the reference function (6.13) becomes

$$
T=273.15+\sum_{i=1}^{20} A_{i}\left(\ln W^{*}(T)\right)^{i}
$$

Procedures for obtaining the calibration constants for the polynomial equations will be described later in this section.

The calculation of $W^{*}(t)$ must be carried out with a large number of digits. At NBS approximately 17 decimal digits are used. A table of $W^{*}(t)$ at closely spaced values of $t$ is given in Appendix E together with a short Fortran subroutine which will produce this table efficiently (no more than two iterations per entry).

The following procedure may be employed to convert the measured values of $W(t)$ to $t$. From eq $(6.13)$ is obtained

$$
W^{*}(t)=W(t)-\Delta W(t) .
$$

When eqs (6.14) and (6.13b) are combined,

$$
t=\sum_{i=1}^{20} A_{i} \ln [W(t)-\Delta W(t)]^{i}
$$

To solve for $t$, an approximate value of $t$ is first obtained by neglecting $\Delta W(t)$ and evaluating eq (6.15) with the measured $W(t)$ only. (A closely spaced table of $W^{*}(t)$ could be employed for this evaluation.) The approximate value of $t$ is used with the appropriate polynomial equation to obtain an approximate value of $\Delta W(t)$ which is then employed with $W(t)$ in eq (6.15) (or table of $W^{*}(t)$ ) to obtain $t$. The process is repeated until the value of $t$ no longer changes more than the desired limits.

Considering the complexity of the reference function (eq (6.14)), a high speed electronic computer or a table compiled by such a computer at small enough temperature intervals $\left(0.1\right.$ to $1{ }^{\circ} \mathrm{C}$, depending upon the value of $t$ ) to permit linear interpolation is a necessity.

Although a table of $W^{*}(t)$ could be employed to obtain $t$ from the measured value of $W(t)$ in accordance with the procedure outlined above, a direct interpolation in a table of $t$ versus $W(t)$ (measured) is obviously more convenient. Such a table can be generated from the relation given by eq (6.13). Values of $\Delta W(t)$ can be calculated at appropriate values of $t$, using the polynomial equations obtained from calibration, and combined with the corresponding values of $W^{*}(t)$ to obtain $W(t)$. (For tables furnished by NBS, see Appendix J.)

\subsection{1. $-182.962{ }^{\circ} \mathrm{C}(90.188 \mathrm{~K})$ to $0^{\circ} \mathrm{C}(273.15 \mathrm{~K})$}

The polynomial deviation function specified by the text of the IPTS -68 between $-182.962{ }^{\circ} \mathrm{C}$ $(90.188 \mathrm{~K})$ and $0{ }^{\circ} \mathrm{C}(273.15 \mathrm{~K})$ is:

$$
\Delta W(t)=W(t)-W^{*}(t)=A_{4} t+C_{4} t^{3}(t-100) .
$$

The constants $A_{4}$ and $C_{4}$ in eq (6.16) are determined by calibration measurements at the steam point, the triple point of water, and the boiling point of oxygen, or alternatively, at the zinc point, the tin point, the triple point of water, and the boiling point of oxygen. Calibration measurements at the oxygen point and again at the TP are added to the sequence of measurements described earlier (sec. 6.1) for the range 0 to $630.74{ }^{\circ} \mathrm{C}$. The value of $W(t)$ at the oxygen point is obtained from $R$ (oxygen) and $R(0)$. The value of $\alpha$ (see eq 6.3)) is required from the measurements above $0{ }^{\circ} \mathrm{C}$ in order to evaluate the constant $A_{4}$ of eq (6.16). From the definition of $\alpha$,

and

$$
\alpha^{*}=\left(W^{*}(100)-1\right) / 100
$$

hence,

$$
\alpha=(W(100)-1) / 100 ;
$$

$\Delta W(100)=W(100)-W^{*}(100)=100\left(\alpha-\alpha^{*}\right)$.

(The $\alpha^{*}\left(=0.0039259668{ }^{\circ} \mathrm{C}^{-1}\right)$ is the value used in forming the $W^{*}(t)$ table [51].) Substituting eq (6.19) in eq (6.16) with $t=100^{\circ} \mathrm{C}$, there is obtained

$$
A_{4}=\alpha-\alpha^{*}=\Delta W(100) / 100 .
$$

The value of $C_{4}$ is determined by measurement of $W(t)$ at the boiling point of oxygen. The deviation $\Delta W(t)=W(t)-W^{*}(t)$ at the oxygen point and the value of $A_{4}$ from (6.19) gives the constant $C_{4}$

$$
C_{4}=\frac{\Delta W(t)-A_{4} t}{t^{3}(t-100)}
$$

where $t$ is the oxygen normal boiling point temperature in ${ }^{\circ} \mathrm{C}$.

The first derivative of the IPTS-68 reference function (eq (6.14)) has the same value at $0^{\circ} \mathrm{C}$ as that of the function $W(t)$ obtained from eqs (6.1), (6.2), and (6.3) with the thermometer constants $\alpha=$ $3.9259668 \times 10^{-3}{ }^{\circ} \mathrm{C}^{-1}$ and $\delta=1.496334{ }^{\circ} \mathrm{C}$. On the other hand, the first derivative of the temperatureresistance function for real SPRT's is not continuous through $0{ }^{\circ} \mathrm{C}$. The discontinuity is, however, very small and may be neglected. See Appendix $F$ for detailed discussion of this point. 


\subsection{1 to $90.188 \mathrm{~K}$}

From 13.81 to $90.188 \mathrm{~K}$, the SPRT's that are received at the NBS are not calibrated at the defining fixed points established by phase equilibrium in accordance with the text of the IPTS -68 , but they are calibrated by a procedure that is equivalent to the IPTS-68. The SPRT's are calibrated at NBS by intercomparison with SPRT standards that maintain the NBS-IPTS-68 (see sec. 7 for details). The temperatures of the intercomparison calibration measurements have been selected so that the measurements, which are made at temperatures very close to those of the defining fixed points, are supplemented by measurements suitably placed between these points. Usually sixteen measurements are made between 12 and $90 \mathrm{~K}$. The data are analyzed by a method of least squares and values of resistances corresponding to the defining fixed-point temperatures are evaluated. The values of $W(T)$ are then computed for the defining fixed-point temperatures: 13.81, $17.042,20.28,27.102,54.361$, and $90.188 \mathrm{~K}$, and applied to eq $(6.13)$ or $(6.13 \mathrm{a})$ to obtain the deviation $\Delta W(T)$ at each of these temperatures. The discussion to follow describes procedures for obtaining the constants of the specified polynomial deviation functions.

Below the oxygen point the deviation, $\Delta W(T)$, of the value of $W(T)$ from the reference function, $W^{*}(T)$, is defined with a succession of polynomial equations, each covering a relatively short range. Each of these deviation equations is described by a three or four term power series in $T$ (where $T$ is the value of temperature on the International Practical Kelvin Scale: $T=t+273.15 \mathrm{~K}$ ) and is a smooth downward extension of the deviation curve found for the temperature range immediately above.

6.2.2.1. 54.361 to $90.188 \mathrm{~K}$

In the range from the oxygen normal boiling point $(90.188 \mathrm{~K})$ down to the oxygen triple point $(54.361 \mathrm{~K})$ the deviation function is

$$
\Delta W(T)=A_{3}+B_{3} T+C_{3} T^{2} .
$$

The three constants $A_{3}, B_{3}$, and $C_{3}$ are determined from the values of $\Delta W(T)$ at the two oxygen fixed points and the value of the first derivative of the deviation equation (6.16) specified for above the oxygen point,

$$
\left(\frac{d \Delta W(t)}{d t}\right)_{0_{2}}=A_{4}-300 C_{4} t^{2}+4 C_{4} t^{3}
$$

where the $t$ in eq $(6.23)$ is the value of the oxygen normal boiling point temperature $\left(-182.962{ }^{\circ} \mathrm{C}\right)$. At the oxygen point the first derivative of the deviation function (eq (6.22)) extending below the oxygen boiling point is set equal to the first derivative of the deviation function (see eqs (6.16) and (6.23)) above the oxygen normal boiling point; i.e.,

$$
\frac{d \Delta W(T)}{d T}=B_{3}+2 C_{3} T=\left(\frac{d \Delta W(t)}{d t}\right)_{0_{2}},
$$

at $t=-182.962{ }^{\circ} \mathrm{C}$ and $T=90.188 \mathrm{~K}$. Thus, the deviation functions and their first derivatives are forced to be equal at the point of joining.

\subsubsection{20.28 to $54.361 \mathrm{~K}$}

The deviation function defined by eq (6.22) is joined smoothly at the oxygen triple point $(T=54.361 \mathrm{~K})$ in a completely analogous manner as described in section 6.2.2.1, with the deviation function between 20.28 and $54.361 \mathrm{~K}$ given by

$$
\Delta W(T)=A_{2}+B_{2} T+C_{2} T^{2}+D_{2} T^{3} .
$$

The constants are determined from the values of $\Delta W(T)$ at the normal boiling points of equilibrium hydrogen $(20.28 \mathrm{~K})$ and neon $(27.102 \mathrm{~K})$ and the triple point of oxygen $(54.361 \mathrm{~K})$ and by equating its derivative and the derivative of eq $(6.22)$ at the point of joining $(54.361 \mathrm{~K})$.

$$
\text { 6.2.2.3. } 13.81 \text { to } 20.28 \mathrm{~K}
$$

A fourth deviation function

$$
\Delta W(T)=A_{1}+B_{1} T+C_{1} T^{2}+D_{1} T^{3}
$$

covers the range 13.81 to $20.28 \mathrm{~K}$. The constants are determined from the values of $\Delta W(T)$ at the triple point $(13.81 \mathrm{~K})$ and the normal boiling point $(20.28 \mathrm{~K})$ of equilibrium hydrogen and at the temperature of $17.042 \mathrm{~K}$ (boiling point of equilibrium hydrogen at $25 / 76 \mathrm{~atm})$ and by equating the derivative of eq (6.26) and the derivative of eq (6.25) at the point of joining $(20.28 \mathrm{~K})$.

\subsubsection{Calibration Tables Between 13.81 and $273.15 \mathrm{~K}$} $\left(\mathbf{O}^{\circ} \mathrm{C}\right)$

At NBS, the coefficients of the deviation functions obtained through the procedure outlined above allow the values of $\Delta W(T)$ to be calculated over the temperature range specified for each of the deviation functions. Values of $W(T)$ for the thermometer being calibrated are then given by

$$
W(T)=W^{*}(T)+\Delta W(T) .
$$

Values are calculated to provide tables of $W(T)$ or $R(T)$ versus $T$ at equal temperature intervals sufficiently small to permit linear interpolation. In the region 13 to $90 \mathrm{~K}$ the tabulations are usually given at $0.1 \mathrm{~K}$ intervals and from 90 to $273 \mathrm{~K}$ at $1 \mathrm{~K}$ intervals.

\subsection{Errors of Temperature Determinations}

The usefulness of a measured value of temperature depends strongly upon both the amount of its 
uncertainty and the knowledge of the amount of its uncertainty. Reduction of the errors contributing to the uncertainty is primarily limited by the ingenuity of the experimentalist; there is, however, an inherent source of uncertainty or spread of temperature values on any practical scale which is predicated upon the use of real materials, even if "perfectly" calibrated thermometers are used and no error is introduced in the measurements. Unfortunately only scattered data are available from which one could infer the degree of this ambiguity or possible spread of values of the defined IPTS- 68 . McLaren [33] did not find a distinotive difference among the measured temperature values at 321 and $231{ }^{\circ} \mathrm{C}$ of eleven thermometers with $\alpha$ ranging from $0.003921{ }^{\circ} \mathrm{C}^{-1}$ to $0.003926{ }^{\circ} \mathrm{C}^{-1}$. (Five of the eleven thermometers met the requirements of IPTS-68, i.e., $\alpha$ equal to or greater than 0.0039250 ${ }^{\circ} \mathrm{C}^{-1}$.) The sensitivity of McLaren's test was about $\pm 0.5 \mathrm{mK}$; his results indicate a spread of temperature values which are an order of magnitude smaller than the spread reported earlier for seven thermometers of much less pure platinum ( $\alpha$ from $0.003909{ }^{\circ} \mathrm{C}^{-1}$ to $0.003925{ }^{\circ} \mathrm{C}^{-1}$ ) by Hoge and Brickwedde [29]. These latter thermometers, while acceptable on the ITS- 27 which was in use at the time of their work, were not made of sufficiently pure platinum to meet the requirement of the IPTS -68 that $\alpha$ equal or exceed $0.0039250{ }^{\circ} \mathrm{C}^{-1}$. The thermometers that were investigated by Hoge and Brickwedde showed a spread of $7 \mathrm{mK}$ between -190 and $0{ }^{\circ} \mathrm{C}$, and spread of $1.3 \mathrm{mK}$ between 0 and $100{ }^{\circ} \mathrm{C}$. The six thermometers measured between 100 and $444{ }^{\circ} \mathrm{C}$ had a maximum spread of $12 \mathrm{mK}$. Data which would indicate the spread of interpolated values of temperature on the IPTS -68 below ${ }^{\circ} \mathrm{C}$ due to the variations in presently acceptable platinum wire is sparse and inadequate [11, 36, 44]. On the basis of existing data, Bedford and Ma [9] estimated that the IPTS-68 is reproducible to $\pm 3 \mathrm{mK}$ between 14 and $20 \mathrm{~K}, \pm 1 \mathrm{mK}$ between 20 and $54 \mathrm{~K}, \pm 2 \mathrm{mK}$ between 54 and $90 \mathrm{~K}$, and $\pm 5 \mathrm{mK}$ bet ween 90 and $273 \mathrm{~K}$. It should be pointed out that, in addition to unaccounted for variations in the platinum wire, a spread in the temperature values may also arise from incomplete definitions of the materials that define fixed points (e.g., their isotopic composition).

The remainder of this section will deal with errors that arise both from errors of measurement by the user and from errors of calibration of the thermometer.

Many of the same kinds of error sources plague both the calibrator and the user. The errors may be divided into two categories; first, a temperature error, i.e., a difference between the actual thermometer temperature and the temperature of the point of interest or reference temperature, and second, a resistance error, i.e., a difference between the measured resistance and the resistance of the sensor. For example, temperature error occurs because of an unknown difference between the temperature of the thermometer and the equilibrium temperature realized in a fixed-point apparatus used for calibration. (The calibrator is also plagued, of course, by any unknown difference between the equilibrium temperature realized in his fixed-point apparatus and the equilibrium temperature defined by IPTS-68.) Temperature error also occurs because of unknown temperature differences between the thermometer and the object of interest in the user's apparatus. The experimenter must decide where to place the thermometer to minimize the magnitude of this error. Several possible sources of temperature error are discussed in section 4 . In considering the errors of thermometer resistance measurements one must not only examine the reproducibility and calibration of his measurement equipment but also his measurement technique. Sections 5,7 , and 4 indicate possible sources of resistance or resistance ratio error.

The following analysis describes the error in the calculated value of temperature that results from errors made in calibrating the SPRT at the fixedpoints. Any additional imprecision and inaccuracy introduced by the user are assumed to be best known by him.

At any value of temperature, $t$, the total differential of $t$ for the SPRT is,

$$
d t=\sum_{i}\left(\frac{\partial t}{\partial t_{i}}\right) d t_{i}+\sum_{i}\left(\frac{\partial t}{\partial W_{i}}\right) d W_{i}
$$

where $t_{i}$ is the temperature attributed to the calibration fixed point and $W_{i}$ is the value of the resistance ratio, $R\left(t_{i}\right) / R(0)$, attributed to that temperature. The analysis may be simplified by converting any error in the value of temperature attributed to the calibrating fixed point to an equivalent error in $W$ at that fixed-point; then, $d t_{i}$ of eq (6.27) vanishes and eq $(6.27)$ reduces to

$$
d t=\sum_{i}\left(\frac{\partial t}{\partial W_{i}}\right) d W_{i}
$$

where $d W_{i}$ now includes the contribution from errors in the values of temperature as well as the errors in the values of $R(t) / R(0)$ at the calibration points. By utilizing eq $(6.28)$ the error in the value of temperature $t$ on the IPTS-68 caused by an error in $W_{i}$ corresponding to a positive unit error in $t_{i}$ was evaluated as a function of temperature. Figure 17 shows the errors in the values of temperature that would result from the calibration errors at each of the fixed points. For each curve the calibration error is taken to have occurred at only one fixed point with no calibration error at the other fixed points.

The measurement at the triple-point of water should be a part of platinum resistance thermometry work and $W(t)$, i.e., $R(t) / R(0)$, should be used in calculating temperatures (see sec. 6.1). The value 


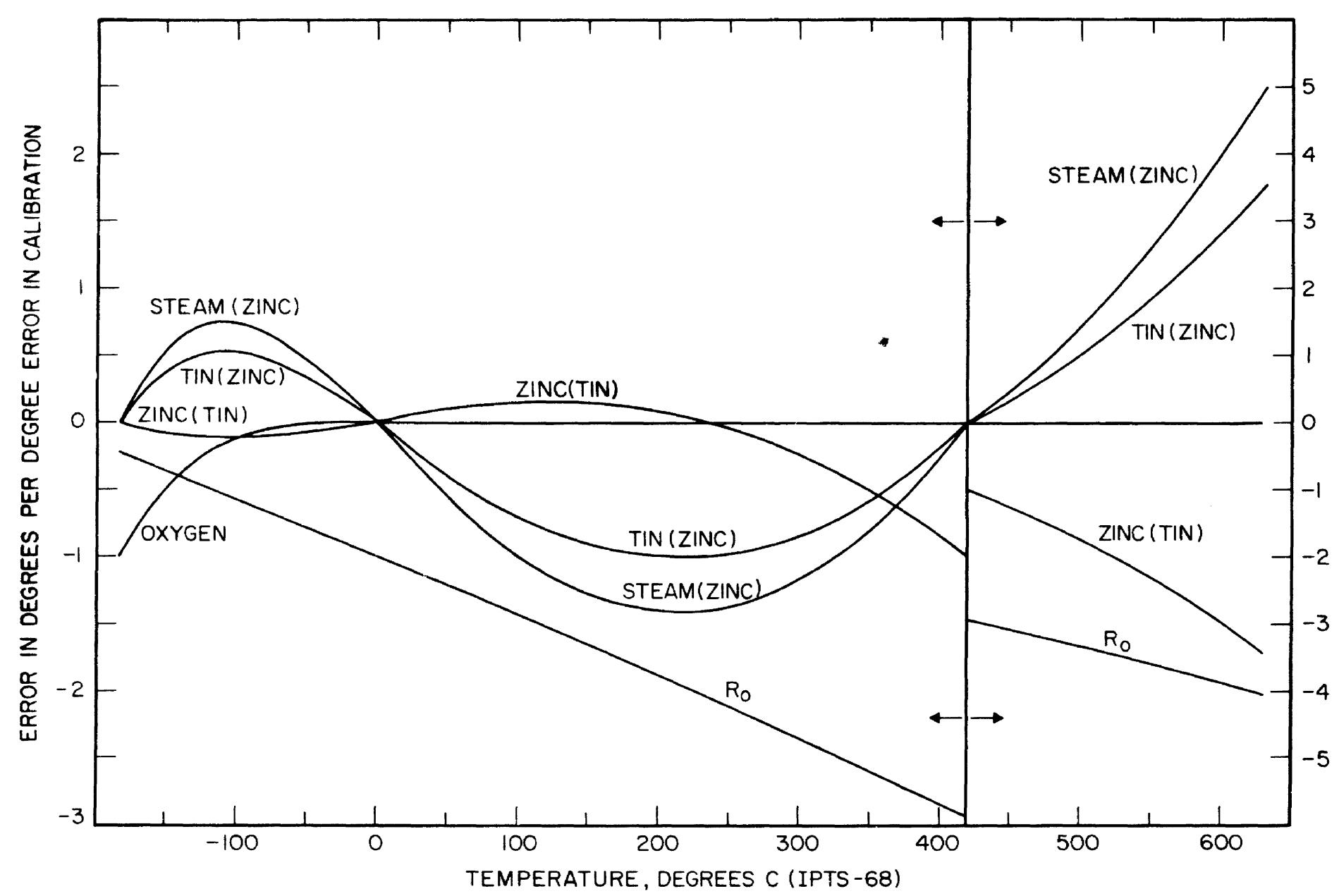

FIGURE 17. The error at various temperatures propagated from errors made in the calibration of a platinum resistance thermometer.

The curves show the error (i.e.. departure from the IPTS-68) in the values of temperatures caused by a unit positive (hotter) error in realizing the temperature (hotness) of one given calibration point. The calibration at the triple point of water is assumed to have been made without error. The fixed point at which the error was made is indicated on the curve The error curve depends not only upon the particular fixed point at which the error occurred, but also upon which other fixed points above $0^{\circ} \mathrm{C}$ were employed in the calibration. The calibration at the other fixed point above $0^{\circ} \mathrm{C}$, indicated in parenthesis, is assumed to have been performed without error. A calibration error at the oxygen normal boiling point does not introduce an error in the measured values of temperature above $0^{\circ} \mathrm{C}$. The first derivatives of the ZINC(TIN) curve and the TIN(ZINC) curve are not continuous through

The curve marked $R_{0}$ shows the error that would be introduced if the experimenter makes a unit positive error in realizing the temperature (hotness) of $0{ }^{\circ} \mathrm{C}$ and then calculates the value of a temperature from the value of $R(t) / R\left(0^{\circ} \mathrm{C}\right)$

of $W(t)$ is particularly sensitive to the errors of $R(0)$ in applications at high temperatures where any error of $R(0)$ becomes amplified. Figure 17 shows the error curve resulting from an error in $R(0)$ corresponding to a positive unit error in temperature.

The estimated uncertainties of the calibration measurements obtained at NBS are 0.002 or $0.003 \mathrm{~K}$ at the oxygen normal boiling point, $0.0002 \mathrm{~K}$ at the triple point of water, $0.001 \mathrm{~K}$ at the tin point, and $0.001 \mathrm{~K}$ at the zinc point. These uncertainties are estimates; hopefully, in the future, all of the desired documentation of these estimates will become available. The experimenter could estimate his overall error by statistically summing his measurement error with the calibration errors that can be obtained from the curves on figure 17 and the above estimate of calibration uncertainties. (For further details see Appendix G.)

\section{Calibration}

The calibration of a SPRT on the IPTS- 68 involves the measurement of the resistance when the thermometer is at the temperature of the prescribed defining fixed points. This section deals with the equipment, the preparation of fixed-point cells, and the procedures employed at NBS to realize the temperatures of the prescribed defining fixed points for calibrating SPRT's.

In practice, thermometer calibrations are not made at exactly the temperatures of prescribed equilibrium conditions for the fixed points. For small known departures from the prescribed conditions a temperature correction can satisfactorily be made, e.g., such a departure may exist because of the hydrostatic head at the level of the thermometer resistor in calibrations that employ triple-point or freezing-point cells. (The effect of pressure devia- 


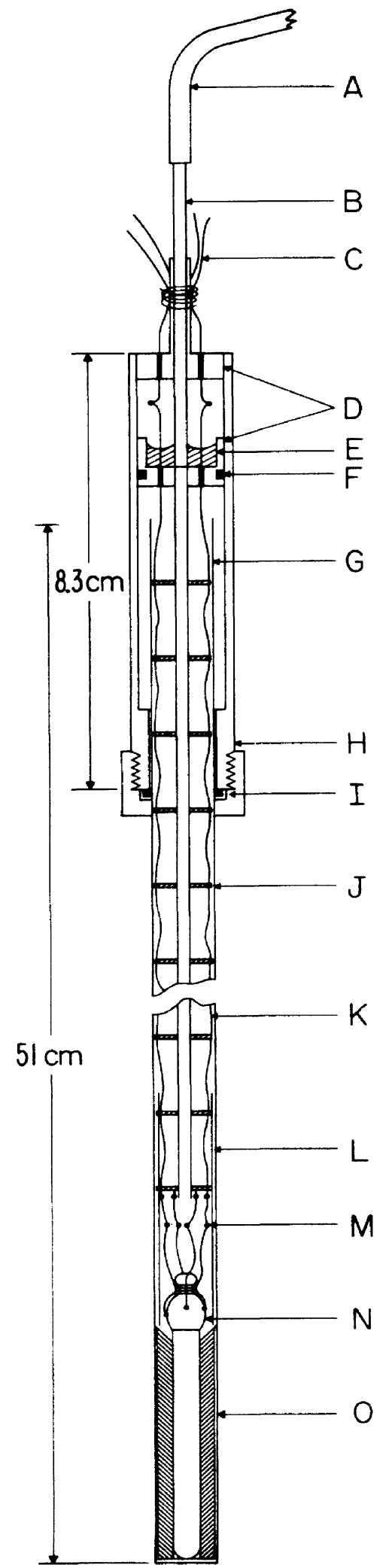

FiguRE 18. Holder for capsule-type platinum resistance thermometers.

Calibration measurements are performed in the holder at the triple point of water, tin point, and the normal boiling point of oxygen.

A. Elastomer tubing to helium gas source.

B. Thin $(0.005$ ") wall stainless steel tubing for purging the holder with helium gas before the vacuum tubing connector $(I)$ is sealed.

C. Leads to measurement equipment (Mueller bridge)

D. Sections of mechanical tie-down (brass), soldered to the stainless purge tube, for guiding and fastening the incoming thermometer leads.

E. Hard wax for holding and sealing the $0.005^{\prime \prime}$ gold leads that extend down to the ther mometer. tions is given in the text of IPTS-68, Appendix A.) Gross departures from the prescribed conditions usually require extensive efforts to establish that the adjusted temperature and resulting calibration are sufficiently close to the IPTS -68 .

The calibration apparatus at the NBS has been designed to be used with the great majority of SPRT's. Capsule type SPRT's, however, are mounted in special stainless-steel holders (fig. 18) before they are calibrated in fixed-point equipment with deep thermometer wells.

\subsection{Triple Point of Water}

The triple point of water $\left(0.01{ }^{\circ} \mathrm{C}\right)$ is the most useful and important of the defining fixed points for calibrating SPRT's. The virtues of regular thermometer measurements at the triple point of water are so great that all but the most casual measurements of temperature with a SPRT should include a reference measurement at the triple point. The triple point is realized in a sealed glass cell (fig. 19) containing ice, water, and water vapor. When the cell is in use it may be placed in an ordinary crushed ice-water bath. Figure 19 shows the cell and icebath system used at NBS.

The cell is first immersed in the ice bath with the mouth of the re-entrant thermometer-well above the surrounding ice-water level. The well is thoroughly dried, then filled with crushed Dry Ice ${ }^{2}$ and maintained full for about $20 \mathrm{~min}$ by replacing the sublimated Dry Ice. The initial freezing of water within the triple-point cell will occur several degrees below the triple-point temperature because water easily supercools. When the water does start to freeze fine needles of ice crystals (dendrites) are initially formed and protrude from the wall of the well into the liquid. The fine needles quickly cover the well but soon disappear to form a clear coating of ice (on the well) that will grow and become a 4 to $8 \mathrm{~mm}$ thick mantle in about $20 \mathrm{~min}$. It is important to keep the well completely full of Dry Ice during this period. (If the Dry Ice level in the well is allowed to drop several inches and then the well is refilled, the ice mantle is very apt to crack. The desired triplepoint temperature may not be achieved if a crack

\footnotetext{
${ }^{2}$ Certain commercial materials are identified in this paper in order to adequately specify the experimental procedure. Such identification does not imply recommenda tion or endorsement by the National Bureau of Standards.

F. "O" ring vacuum seal to brass tube $(H)$

G. Thin wall stainless steel tube $\left(7 / 16^{\prime \prime}\right.$ od.) closed at the bottom.

H. Brass tube with lead seal at top and vacuum tubing connector $(I)$ at the bottom.

I. Vacuum tubing connector for sealing the tube $(G)$

J. Polytetrafluoroethylene plastic lead spacers.

. Insulated gold leads (4) passing through holes close to the outer diameter of the spacers $(J)$ to attain good tempering. The lead insulation (not shown) is polytetrafluoroethylene tubing cut into a helix and held in tension to eliminate buckling. The four gold leads are welded at the bottom end to short sections of platinum leads.

L. Thin polytetrafluoroethylene sheet rolled into a cylinder to insulate the exposed leads (near the connections to the capsule thermometer) against the stainless steel tubing.

M. Connections between the short sections of platinum leads of the holder and the platinum leads of the capsule thermometer.

Capsule thermometer.

$O$. Aluminum sleeve to fit the thermometer and the stainless steel tube. The sleeve reduces the external heating effect of the thermometer.
} 
in the ice mantle extends from the well surface into the surrounding liquid water.) In the process of introducing Dry Ice into the well some of the Dry Ice may be deposited around the top of the cell, causing the water within the cell to freeze solidly across the top. The ice at the top of the cell should be melted immediately to avoid the possible breaking of the cell glass. Whenever ice is frozen solidly across the top surface of the cell water and a strong bond is formed between the thermometer well and the outer cell wall, any subsequent freezing of water below the surface ice can result in sufficient pressure to rupture the cell. The surface ice can be melted by raising the cell slightly and warming the top of the cell with the hands briefly while gently shaking the top of the cell sideways to "wash" the region with the cell water which facilitates the melting of the layer of ice. After $20 \mathrm{~min}$ no additional Dry Ice should be added and the remaining Dry Ice in the well should be allowed to sublime completely. Finally, when the Dry Ice in the well is completely gone, the cell is lowered deeper into the ice bath and the well allowed to fill with water. If the cell is raised high enough to see the mantle during the freezing process the magnification of the cylinder of water will give the impression that the cell is or is about to be frozen solid with ice although the coating of ice on the well may still be as little as 1 or $2 \mathrm{~mm}$ thick. If the cell is inverted, the true thickness of the ice may be seen. (The cell should not be inverted after the "inner-melt," described in the next paragraph, has been made.) An immersion type cooler may be used instead of Dry Ice for freezing the ice mantle [25]. However, care must be taken to avoid admitting the auxiliary heat-transfer liquid, e.g., alcohol, into the surrounding ice bath.

A second ice-water interface is formed by melting the ice immediately adjacent to the well surface. This is referred to as the "inner melt." The inner melt is made by inserting a glass tube at ambient temperature into the well for a few seconds. A test for the existence of the ice-water interface over the entire interior surface of the mantle is to give the cell a small rotational impulse and determine whether or not the ice mantle rotates freely around the axis of the thermometer well.

Because of some evidence [12] that the temperature of the triple-point cell is sometimes slightly low (the order of $2 \times 10^{-4}{ }^{\circ} \mathrm{C}$ ) immediately after freezing, the cell should be prepared at least one day prior to its use. The reason for this low initial temperature and the subsequent gradual increase during one or two days to a steady value is not clearly established but is believed to be connected with structural strains that are produced when the ice is first frozen; presumably the strains are relieved with time as the ice anneals. The magnitude of the lower initial temperature and the rate of increase to a steady temperature value is dependent upon the specific technique that is employed in freezing the cell.
Figure 19 shows a triple-point cell immersed in an ice bath. In using the cell, a small soft plastic sponge $(J)$ is first placed at the bottom of the well to reduce the mechanical shock that the thermometer might otherwise experience when it is lowered. Also, a closely fitting aluminum bushing (I) about $5 \mathrm{~cm}$ long is placed above the sponge (at the bottom of the well) to reduce the external self heating of

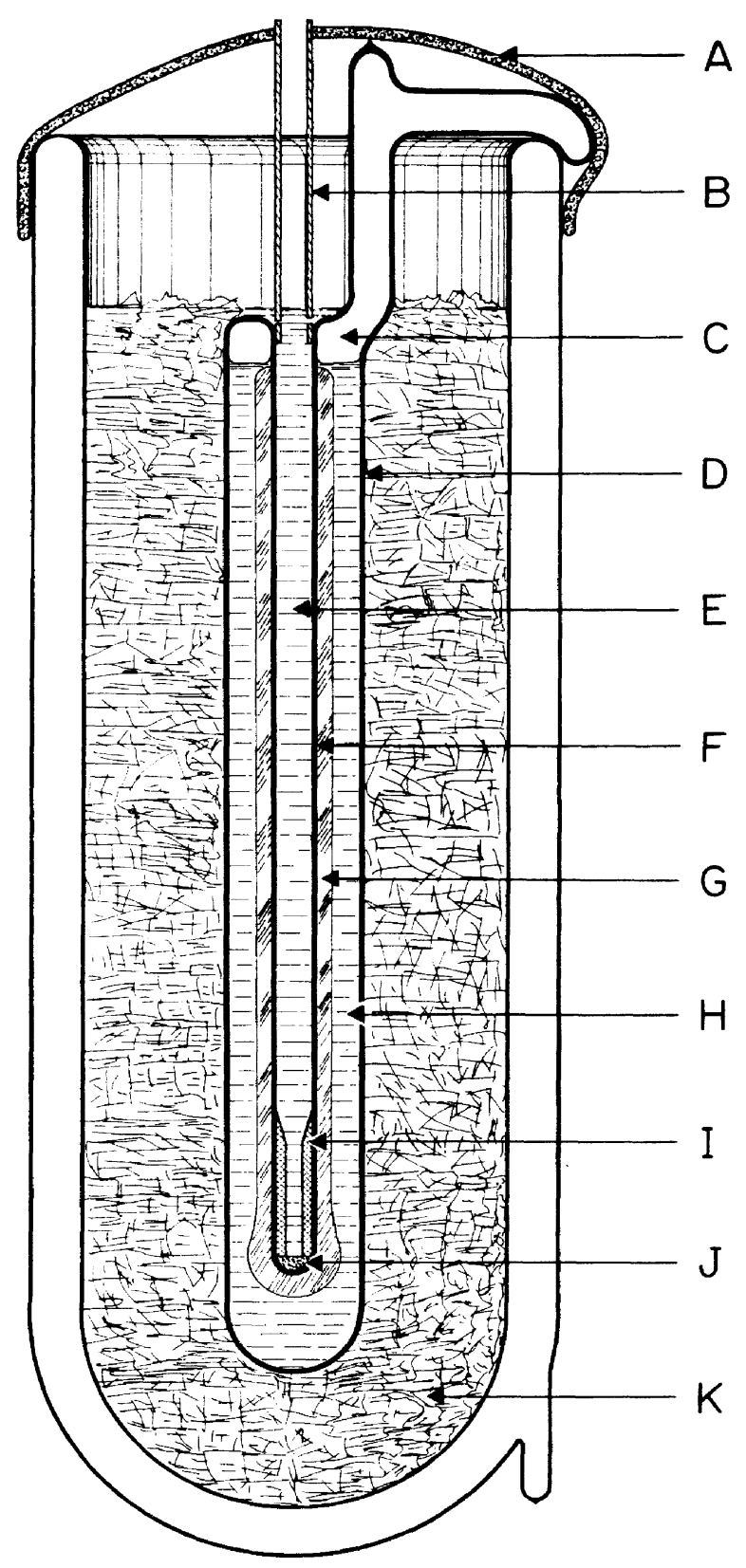

Figure 19. Water triple point cell.

A. Heavy black felt shield against ambient radiation

B. Polyethylene tube for guiding the SPRT into the thermometer well.

C. Water vapor

D. Borosilicate glass cell

E. Water from ice bath.

E. Water from ice bath.

F. Thermometer

H. Air-free water.

I. Aluminum bushing with internal taper at upper end to guide the SPRT into the close-fitting inner bore.

1. Polyurethane sponge.

K. Finely divided ice and water. 
the thermometer. In a half-inch well, filled only with water for thermal contact between the SPRT and the well, the external self heating of a typical $25 \Omega$ SPRT (7.5 mm O.D.) is about $0.2 \mathrm{mK} /(\mathrm{mA})^{2}$. The bushing reduces this heating by a factor of five or more depending upon its fit with the well and the SPRT. (See sec. 4 for the discussion on the self heating of SPRT.) To eliminate the ambient room radiation (from ceiling lights in particular) a heavy black felt cloth (A) covers the top of the cell except for a hole through which the thermometer may be inserted. The thermometer is precooled in the ice bath that surrounds the cell before it is inserted into the cell. A polyethylene plastic tube (B) from the hole in the felt cloth to the re-entrant well provides a guide for inserting the SPRT. Before measurements are made a minimum of five minutes is allowed to elapse (with the thermometer current on and the bridge nearly balanced) for the thermometer to attain thermal equilibrium. If an ice particle is present in the well near the SPRT resistor, an error will occur in the calibration. The water in the well must be free of ice before the SPRT is inserted. Routine measurements made in caljbrating thermometers in triple-point cells have an estimated standard deviation of less than $0.14 \mathrm{mK}$. Very careful work using two currents and extrapolating to the resistance value for zero current has yielded an estimated standard deviation of less than $0.04 \mathrm{mK}$.

\subsection{Metal Freexing Points}

Freezing points are advantageous because the effect of a change in pressure on the temperature, the value of $d t / d p$, is much smaller than that of boiling points. In a metal freezing-point cell, the temperature at the solid-liquid interface depends upon the concentration and kind of impurities; also, strains in the solid and grain size effect the temperature. The concentrations of impurities (solute) existing at the interface depend upon the amount and kinds of impurities in the sample, the amount of sample frozen, and the rate of freezing. To achieve a true temperature and phase equilibrium the net rate of freezing (or melting) must approach zero. This equilibrium condition may be illustrated by the binary composition versus temperature phase diagram shown in figure 20 in which the two constituents are completely miscible in both the liquid and solid phases, e.g., the system $\mathrm{Ag}$-Au or $\mathrm{Bi}-\mathrm{Sb}$. The phase diagram of figure 20 is presented for its simplicity and, since metal samples employed in freezing-point cells are prepared by the zone refining process, the remaining impurities (combined as a single component in the illustration) are expected to form solid solution with the major component. (Most binary metal systems on which data exist do not form a continuous series of solid solutions; various degrees of immiscibility are usually found.) The figure shows the region of the

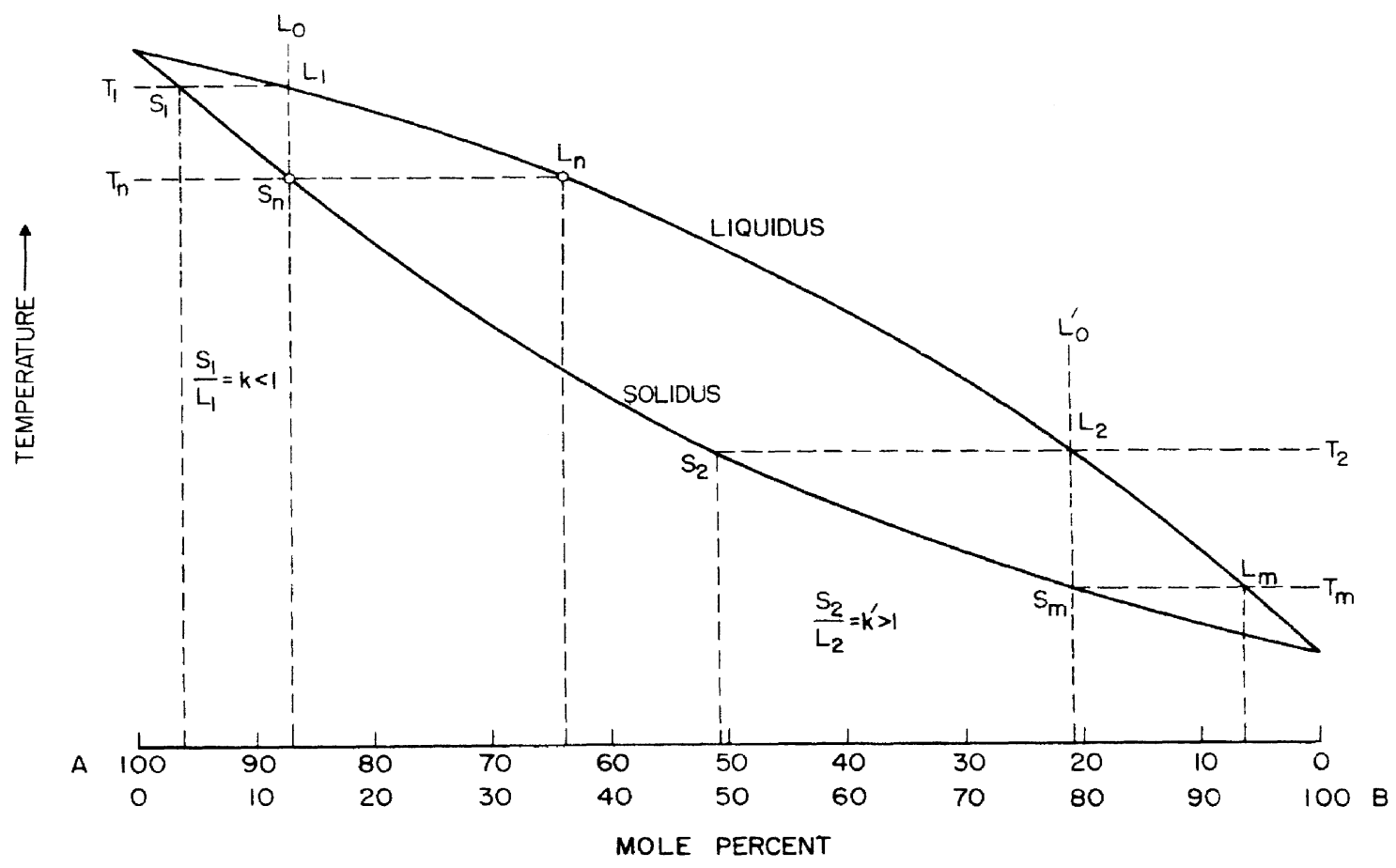

Figure 20 Binary phase diagram of a system that is completely miscible in both solid and liquid phases.

The solidus and liquidus curves represent compositions, respectively, of the solid and liquid phases that can coexist in equilibrium. The left side of the diagram shows that the solute concentration in the solid phase is less than that in the liquid phase, i.e., $k<1$. The right side of the diagram, on the other hand, shows that the solute concentration in the solid phase is greater than that in the liquid phase, i.e., $k>1$. 
composition and temperature where the solid and liquid phases can coexist. The solidus curve indicates the temperature at which a solid solution of any given composition would begin to melt; the liquidus curve indicates the temperature at which a liquid solution of any given composition would begin to freeze (assuming no supercooling). Alternatively, when both solid and liquid phases are present in equilibrium, the composition of each phase is given by the intersections of the temperature line with the solidus and liquidus curves, respectively. An enlarged section of this phase diagram, showing the effect of a small amount of $B$ in nearly pure $A$, is shown in figure 21 a. If a completely melted sample of composition $L_{0}$ is allowed to cool under equilibrium conditions, no change in composition of the liquid occurs until the temperature reaches the liquidus curve $\left(L_{1}\right.$ at temperature $\left.T_{1}\right)$ at which point solid (assuming no supercooling) of the composition $S_{1}$, given by the solidus curve at the same temperature, is formed. In the case shown here, the first solid contains a smaller proportion of $B$ than the liquid from which it was formed; as more solid is formed the concentration of $B$ in the liquid increases. When freezing under equilibrium conditions the composition of the solid phase moves from $S_{1}$ to $S_{n}$; the composition of the liquid phase moves from $L_{1}$ to $L_{n}$. The last of the liquid solidifies at $T_{n}$. Obviously the amounts of $A$ and $B$ in the completely solidified sample must be the same as in the original liquid. $S_{n}$ must, therefore, equal $L_{0}$ and $L_{1}$ and no segregation occurs. The temperature range of the "freeze" is from $T_{1}$ to $T_{n}$. Although in most systems the solutes. lower the freezing point, there are systems in which solutes raise the freezing point; antimony in tin is an example of the latter [30]. Such a system is illustrated by the extreme right portion of the equilibrium phase diagram where $B$ is the major constituent as in figure 20. An enlarged section is shown in figure $21 \mathrm{~b}$. The analysis of the phase diagram is similar to that given previously. The most notable difference is that, as the diagram shows, the concentration of the solute is greater in the solid than in the liquid. The ratio $(S / L)$ of solute concentration in the solid $(S)$ to that in the liquid $(L)$ is called the solute distribution coefficient $(k)$ and, as given in figures 20 and 21 is less than unity for solutes that depress the freezing point and is greater than unity for solutes that elevate the freezing point. Except for very dilute solutions, depicted in figure 21 , the solute distribution coefficient depends on concentration.

Phase diagrams are idealized conceptions of systems at equilibrium (often based on relatively little data). For equilibrium freezing the crystallization process must proceed at such a negligible rate that there is sufficient time for the diffusion of impurities within the solid matrix to achieve uniform distribution throughout the solid, i.e., there is
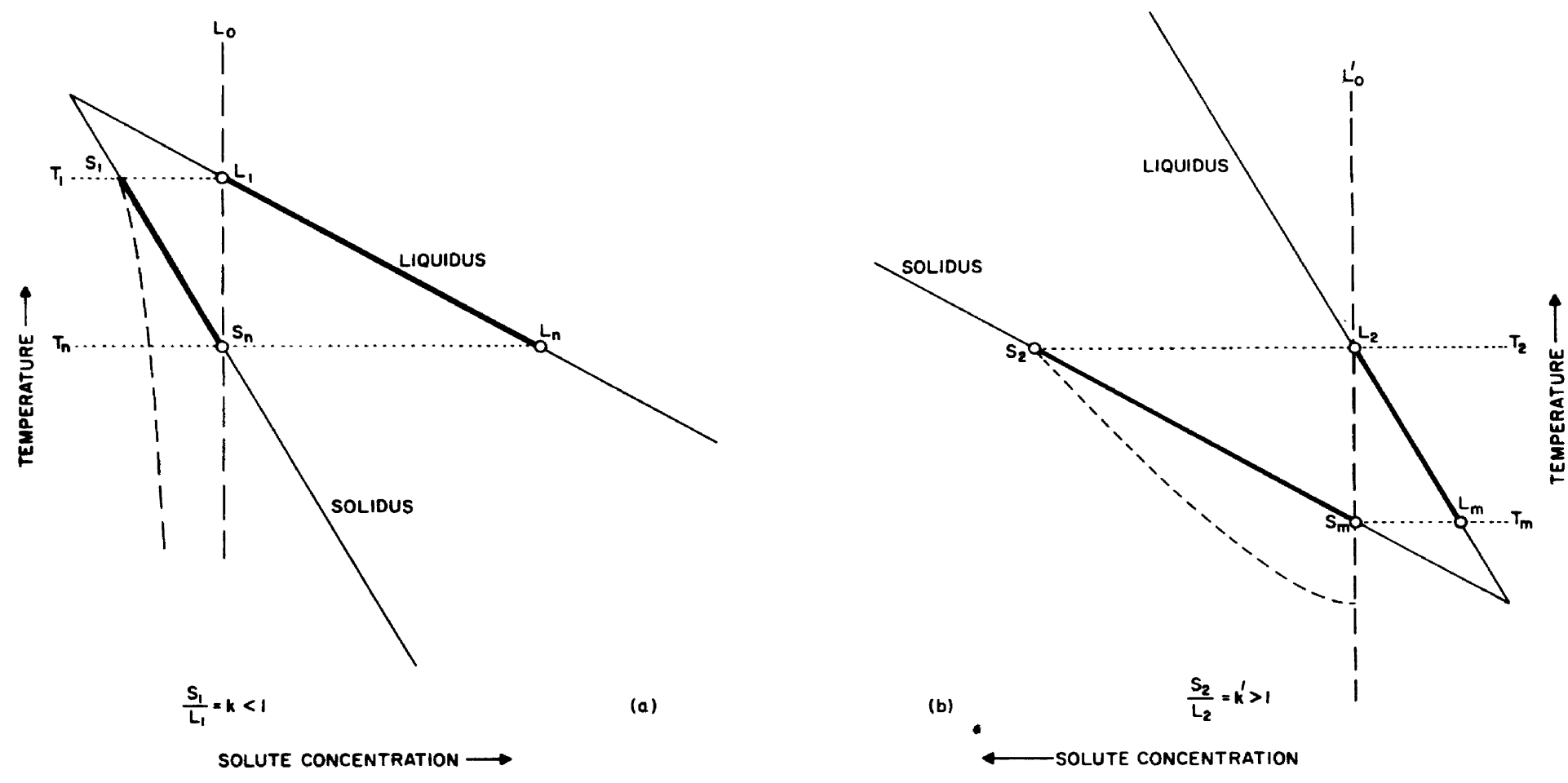

FIGURE 21. Enlargement of a binary phase diagram in the region of high purity of each component.

The solute distribution coefficient $k$ is taken to be constant. Figure (a) represents a phase diagram when $k<1$ and figure (b) when $k>1$.
The heavy lines on the liquidus and solidus curves represent compositions of the liquid and solid phase during the equilibrium freezing process. The dashed line beginning at
in (a) or $S_{2}$ in (b) and extending downward represents the average composition of the solid phase during a semiequilibrium freezing process. The composition of the solid at the $S_{1}$ in (a) or $S_{2}$ in (b) and extending downward represents the average composition of the solid phase during a semiequilibrium freezing process. The composition of the solid at the freezing interface is represented by the solidus curve and that of the liquid phase in equilbnum with the treezing intertace is represented by the liquidus curve. (The solute distribution coefficient may not be constant over the entire range of fraction frozen for semiequilibrium freeze; therefore, eq ( 7.1$)$ may not be valid when $g$ approaches unity.) 
no concentration gradient in the solid. Experimentally this rate of freezing is never realized; however, freezing rates can be achieved which, while large compared to the diffusion rates in the solid matrix, are small compared to the diffusion rate in the liquid. This condition leads to a maximum segregation in the solid but homogeneity in the liquid; this condition will be referred to in this monograph as semiequilibrium freezing. The results of semiequilibrium freezing are shown in the phase diagram of figure 21 and on the cooling curve of figure 22 . The phase diagram still represents the compositions at the solid-liquid interface but the solidus curve no longer is the average composition of the solid phase: the average composition of the solid is given by the dotted line beginning at $S_{1}$ in figure 2la. Compared to an equilibrium freeze, the freezing temperature range for the entire sample is increased due to the increased concentration of impurities at the interface near the end of the freeze. (This assumes no eutectic is formed in the equilibrium freeze.) For the case of semiequilibrium freezing Pfann [40] ${ }^{3}$ gives an expression equivalent to

$$
S / L_{0}=k(1-g)^{k-1},
$$

where $L_{0}$ is the overall solute concentration and $S$ is the solute concentration of the freezing interface after the fraction $g$ of the originai mass of liquid has frozen. (The equation is not applicable for the entire range of $g$. The derivation assumes the distribution coefficient $k$ to be constant and the solute diffusion rate in the solid to be zero.) Figure 23 gives curves of relative solute composition of the freezing interface resulting from this expression for various values of $k$. Figure 22 compares the curve for $k=0.4$, replotted on a linear scale, with the curve that would be obtained for a corresponding equilibrium freeze. The semiequilibrium freeze is shown to have a broader freezing range.

Sufficiently rapid freezing causes a departure from the semiequilibrium freeze. As the velocity of the advancing solid-liquid interface increases, the rate of solute rejection into the liquid at the interface increases. When the impurity can not be uniformly distributed throughout the liquid by either diffusion or convective mixing, the concentration of the solute builds up at the interface as shown in figure $24 \mathrm{~b}$. When this occurs, the effective segregation of the solute decreases (i.e., the effective value of $k$ approaches 1). Because of the increase in the solute concentrations at the interface, the temperature of the interface becomes lower. Figure $24 \mathrm{c}$ shows qualitatively the result of a freeze which is very rapid compared to the impurity diffusion rate in the liquid (and for which there is no other method of homogenization, e.g., stirring).

A notable example of nonequilibrium freezing in metal freezing-point cells is the rapid freezing that

\footnotetext{
3 Pfann [40] refers to semiequilibrium freezing as normal freezing.
}

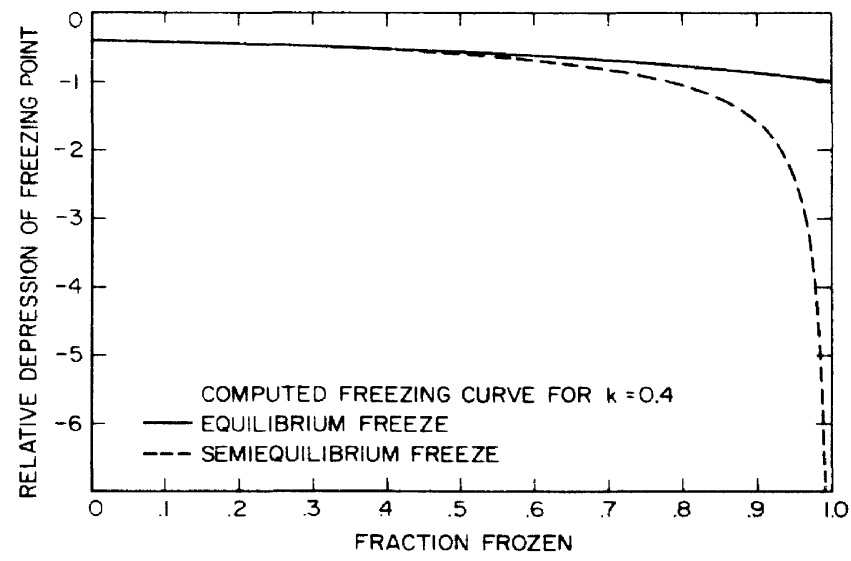

FIGURE 22. Comparison of computed freezing curves for equilibrium and semiequilibrium freezing processes of a completely miscible (in solid and liquid phases) binary system with constant solute distribution coefficient $(k=0.4)$.

The solid line represents the equilibrium freezing process and the dashed line the semiequilibrium freezing process. In the equilibrium freezing process, the temperature, when the last trace of liquid freezes, is shown to be depressed one unit for the "sample": the temperature, when the first solid freezes, is shown to be depressed 0.4 units. The the temperature, when the first solid freezes, is shown to be depressed 0.4 units. The
freezing curve for the semiequilibrium freezing process is shown relative to that of the equilibrium freezing process. The solute distribution coefficient may not be constant equilibrium freezing process. (The solute distribution coefficient may not be constant
over the entire range of fraction frozen for semiequilibrium freeze: therefore, equation (7.1) may not be valid when $g$ approaches unity.)

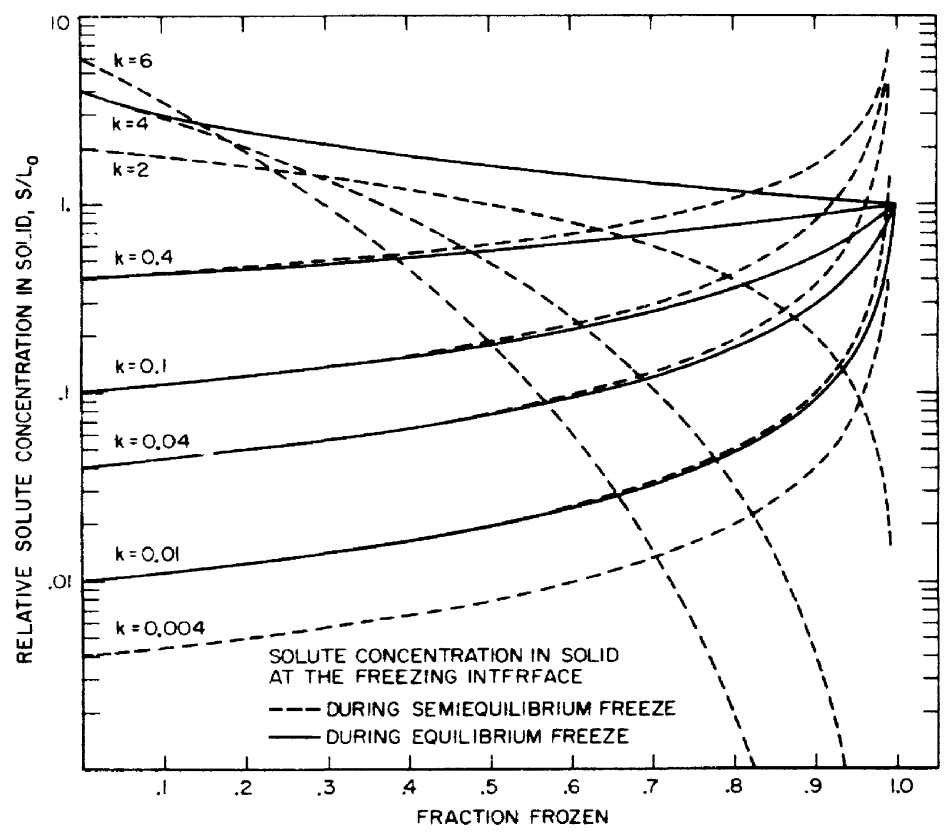

FIGURE 23. Comparison of relative solute concentrations of the solid phase at the freezing interface during equilibrium and semiequilibrium freezing processes of a completely miscible (in solid and liquid) binary system with constant solute distribution coefficient $k$.

Curves are given for various values of solute distribution coefficient. The solid curve represents an equilibrium freezing process and the dashed curve represents $=\mathrm{semi}$ equilibrium freezing process. The solute distribution coefficient may not be constant over the entire range of fraction frozen for semiequilibrium freeze; therefore, eq (7.1) over the entire range of fraction frozen for
may not be valid when $g$ approaches unity.)

occurs after a supercool. When the completely melted sample is cooled through the temperature at which the phase diagram indicates that the 

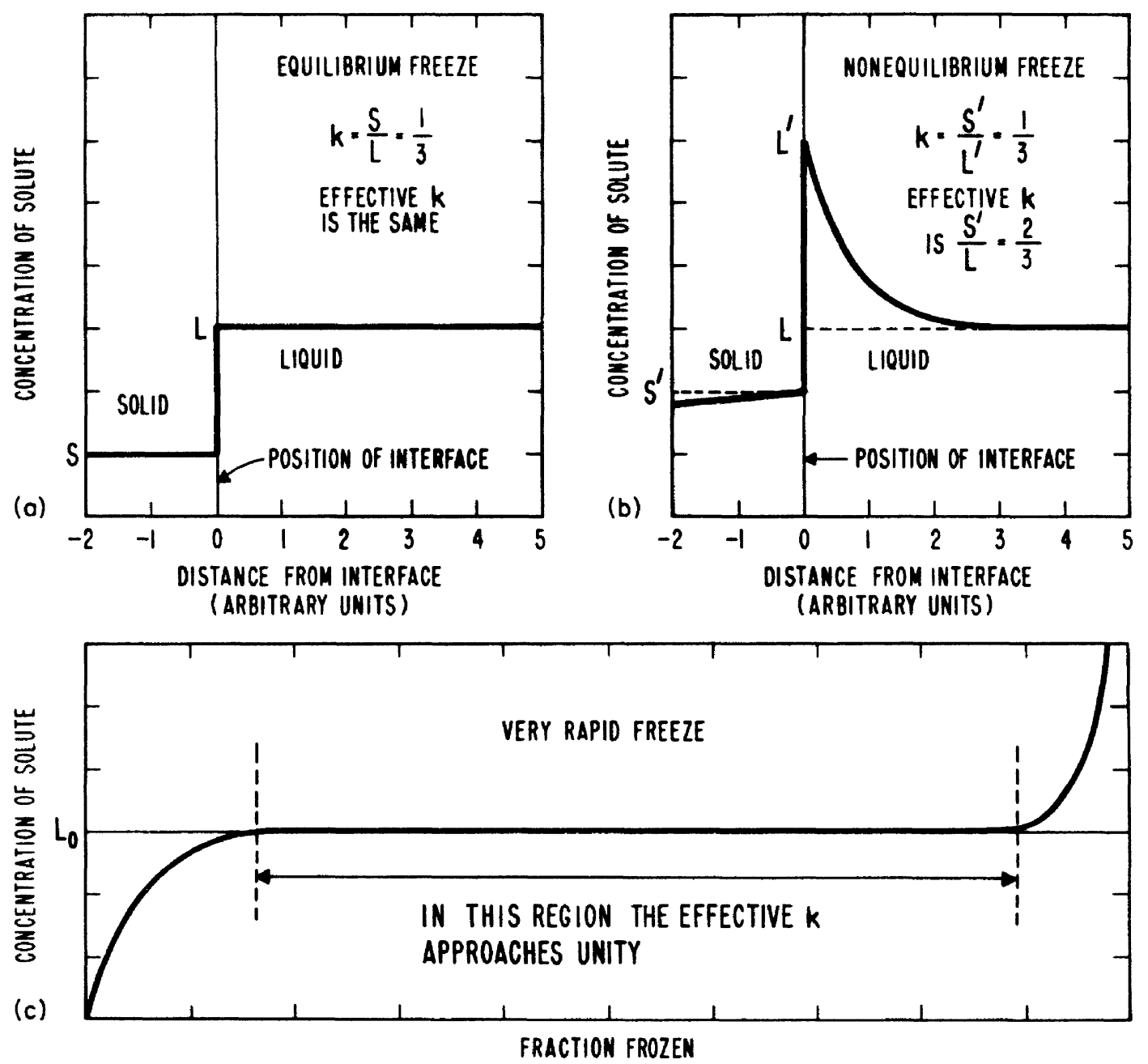

FIGURE 24. Dependence of solute concentration upon the freezing rate.

(a) The concentration of solute in the region near the freezing interface during an equilibrium freeze with the solid-liquid interface advancing at a negligible rate. There are no concentration gradients in neither liquid or solid phases.

(b) The solute concentration near the interface during a nonequilibrium freeze with the interface advancing rapidly relative to the rate of diffusion of the solute in the liquid. The solute concentration in the liquid at the interface increases until at steady state the flow of solute from the freezing liquid to the liquid at the interface equals the flow away from the liquid at the interface to the bulk of the liquid. The solute concentration of the frozen solid is shown to be increasing with the advancing interface.

(c) The solute distribution in the frozen solid approached by a very rapid freeze. The curve shows the initial transient rise of solute concentration of the solid to that corresponding to the solute distribution coefficient of unity.

solid should first appear (i.e., crosses the liquidus curve) no solid appears. The first solid appears at a somewhat lower temperature (typically 0.02 or $0.06{ }^{\circ} \mathrm{C}$ lower in zinc, 1 to $25{ }^{\circ} \mathrm{C}$ lower in tin, water, or antimony). The solid tends to grow most rapidly into the cooler parts of the liquid until the released heat of fusion raises the temperature of the liquid to the equilibrium temperature of the composition at the solid-liquid interface. The temperature to which a liquid supercools is not very reproducible even with the same sample. The amount of supercooling seems to depend on the purity of the sample, the thermal history of the melt, the occurrence of mechanical shock or vibration, and other effects not known or understood. The system may be far from equilibrium during the recovery from the supercool; the solid-liquid interface advances very rapidly and there is very little time for diffusion or convection to homogenize the liquid. As a result, the impurities rejected from the freezing liquid for the case where $k<1$ become relatively concentrated in the liquid in the region of the interface. When the recovery from the supercool is nominally complete, the high concentration of impurities in the liquid at the interface will be reduced by diffusion and the temperature will rise until the flow of impurities from the freezing liquid to the liquid at the interface is equal to the flow away from the interface through the remaining "bulk" liquid.

Rapid freezing implies a rapid transfer of heat. Many authors $[42,45]$ have suggested that it may be necessary for the temperature at the solidliquid interface to be significantly below the equilibrium temperature if freezing is to take place rapidly; i.e., the net rate of freezing is zero at the equilibrium temperature and increases only 
with decreasing solid-liquid interface temperatures. The metal in the two phases at the interface thus constitutes a "proportional" controller, supplying heat on demand and having a temperature "offset" from the equilibrium value if demand exists. This picture is intuitively appealing and is in qualitative agreement with the theory of reaction kinetics. But, the crucial questions would seem to be what is the rate of energy released per unit of temperature departure from equilibrium and how is the measurement of this separated from other events briefly outlined above that occur in nonequilibrium freezing? The difficulty of this measurement has prevented firm establishment of this model.

\subsection{Metal Freezing Point Furnaces}

The metal freezing-point cells are used in furnaces of the design shown in figure 25. At NBS, two furnaces are employed, one for the tin point and the other for the zinc point. Except for the metal sleeves employed at the top section of the furnace core, the two furnaces are the same. The core of the furnace is a stack of three cylindrical coaxial blocks of aluminum (top $(\mathrm{G})$, center $(\mathrm{L})$, and bottom $(\mathrm{T})$ ) that are thermally insulated from each other by Fiberfrax paper and surrounded by a nichrome wire heater (main heater, O) which extends the full length of the three blocks. The heater wire fits closely within each of the two holes of the $51 \mathrm{~cm}$ lengths of two-hole oval alumina tubing. These alumina tubings are very closely spaced around the outer surface of the core (parallel to its axis) and are held tightly against the outside of the blocks with three Inconel "garters." (See the periphery of the furnace core section drawings $1,2,3,4$, and 5.) The garters were made of Inconel wire bent into a form resembling a continuing sine-wave about one centimeter in amplitude and wavelength, then rolled to slightly reduce the thickness, and welded into a ring. The top and bottom blocks each have an additional heater ( $F$ and $U$ ) consisting of nichrome wire that passes through $98 \mathrm{~mm}$ lengths of two-hole, round alumina tubings which were selected to fit closely in the 12 holes of each block. To minimize the thermal time lags, the heater assemblies have small clearances between the heaters and the aluminum core blocks. (The leads extending through the core to the heater of the top core block are gold. The leads to the other heaters are heavy nichrome wire.) Extending down through holes that run the length of the three assembled blocks are six thinwall $(0.13 \mathrm{~mm})$ stainless steel tubes (B) nominally $3.2 \mathrm{~mm}$ in diameter. The tubes pass through 3.22 $\mathrm{mm}$ holes in the end blocks (see $\mathrm{a}$ and $\mathrm{b}$ of figure 25). To permit the holes for these tubes to be accurately positioned through the $30.5 \mathrm{~cm}$ long center block, the center block was made in two cylindrical pieces. The end plate $(Q)$ was attached later to the center core block. Grooves that closely fit the stainless steel tubes were milled on the outside of the inner cylinder which was then fitted tightly inside the second outer cylinder. The two cylinders were "shrunk together", a process that involved precooling the inner cylinder in liquid nitrogen and heating the outer cylinder. The tubes are wells into which thermocouples are placed for controlling the furnace temperature; they also serve as wells for small exploratory resistance thermometers to determine the temperature distribution within the furnace core. The entire core of the furnace, including all three blocks and the heaters, slips into a stainless steel tube (A) $11.4 \mathrm{~cm}$ in diameter with a $0.76 \mathrm{~mm}$ wall. Sheets of mica are wrapped around the main heater near each end to center the core within the tube, to enhance thermal contact between the tube and the main heater near the ends, and to reduce undesirable heat convection currents. The centering leaves a small annular air space between the tube and the alumina insulators of the main heater in the region of the center block; the thermal contact of the end blocks with the surrounding stainless tube serves as "thermal end guards" and reduces the thermal gradients in the middle section of the tube opposite the center block.

The weight of the core rests on a $0.51 \mathrm{~mm}$ wall stainless steel tube (W) $11.4 \mathrm{~cm}$ long and $2.5 \mathrm{~cm}$ in diameter. The space beneath the core is filled with loose Fiberfrax insulation. The outside of the furnace is a $35.6 \mathrm{~cm}$ diameter brass tube with end plates and is filled as indicated in figure 25 with Fiberfrax insulation. A rather widely spaced helical coil of $9.5 \mathrm{~mm}$ copper tubing is soldered to the outside brass tube $(\mathrm{K})$ to permit water cooling; a comparable provision is made for the top end plate. The water cooling is not used when the furnace is at the tin point but is helpful at the zinc point in reducing convection currents around the furnace that cause gradients in the head of the thermometer which, in turn, may result in thermal emf's. The thermal insulation is sufficient so that, even at the zinc point with no water cooling, the outside of the furnace is not "hot to the touch." To achieve further thermal insulation, the furnace core is recessed from the top of the furnace as shown in figure 25. A major path of heat loss from the top of the core is along the $11.4 \mathrm{~cm}$ diameter stainless steel tube that contains the core; to reduce the heat loss the core was recessed and the tube was made relatively longer.

The top core block (G) of the tin furnace was designed to receive an aluminum sleeve $(H)$ $10.2 \mathrm{~cm}$ long that is bored to fit as closely as possible the glass cell that holds the tin sample. By using removable metal sleeves a reasonable range of glass cell diameters can be accommodated while still achieving good thermal contact with the cell above the crucible where the heat shunts are located. The glass cell is of sufficiently uniform diameter to achieve a suitably close fit. In the design, allowance has been made for the differential expansion of the aluminum and the glass cell. (The NBS glass cells are generally ground on the 

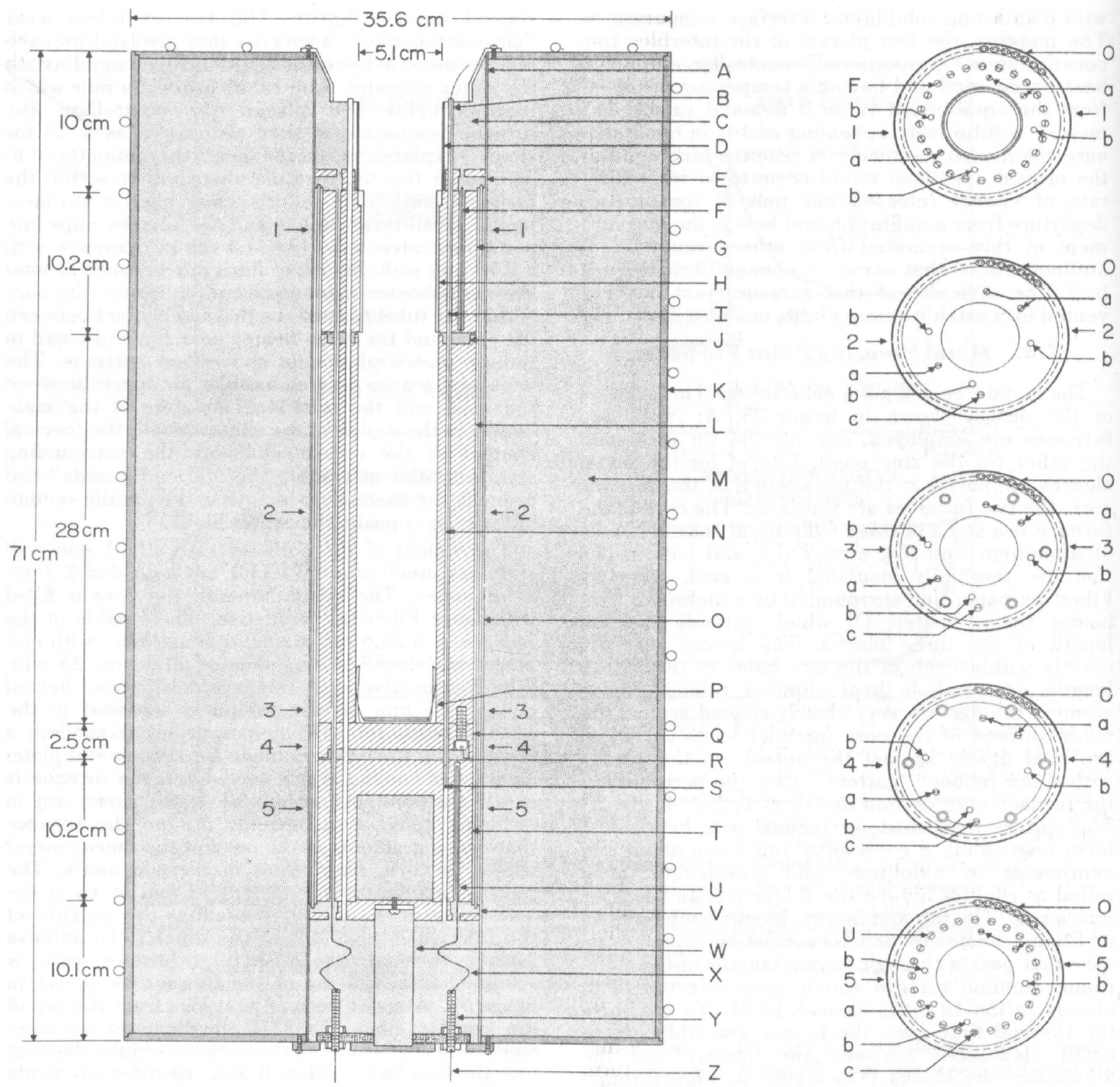

FIGURE 25. Schematic of furnace body and core.

A. Stainless steel tube. $11.4 \mathrm{~cm}$ o.d. $\times 0.76 \mathrm{~mm}$ wall.

B. Stainless steel tubes (6):3.20 $\mathrm{mm}$ od $\times 0.013 \mathrm{~mm}$ wall

P. "Spider" for centering the freezing point cell.

C. Insulation, Fiberfrax mats.

D. Stainless steel tube.

E. Insulation, Fiberfrax mats and mica sheets.

F. Control heaters for top core block.

G. Top core block.

H. Sleeve (aluminum or Inconel) for metal freezing point cell.

I. Elevation of thermocouple junction (Chromel-P/Alumel), top core block. (See a.)

J. Insulation, Fiberfrax sheets.

K. Brass shell, $4.8 \mathrm{~mm}$ thick.

L. Center core block.

M. Insulation, bulk Fiberfrax.

N. Elevation of thermocouple junction (Chromel-P/Alumel), center core block. (See $a$.)

Q. Center core block end plate.

S. Elevation of thermocouple junction (Chromel-P/Alumel) bottom core block. (See $a$.)

T. Bottom core block.

U. Control heaters for bottom core block.

V. Insulation, Fiberfrax mats and mica sheets.

W. Stainless steel tube support, $2.5 \mathrm{~cm}$ sheets.

X. Heater leads.

Y. Posts for heater leads.

Z. To electric power.

a. Wells (B) for control thermocouples (see I, N, and S)

b. Wells (B) for testing the temperature profile of furnace core,

O. Main heaters, held on with Inconel "garters."

c. Leads from top core block heaters.

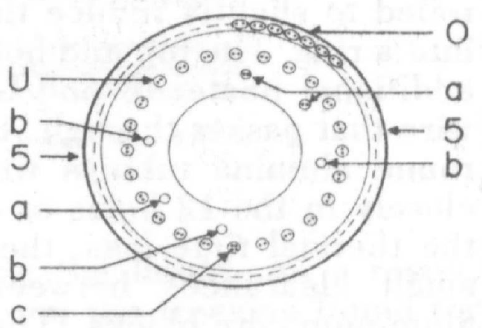


outside to a uniform diameter.) Because aluminum sleeves bond to the aluminum top core block at the zinc point, split inconel sleeves are employed in the zinc point furnace. Also, because of the close tolerances needed for good thermal contact, the zinc-point cells are not permitted to cool to room temperature in the furnace, otherwise the borosilicate glass tube would be crushed owing to the differential contraction between the aluminum top end block and the glass.

The center core block (L) was bored to provide approximately $1.0 \mathrm{~mm}$ clearance for the most common (and largest) size of glass tubes, specifically $51 \mathrm{~mm}$ o.d.; this $1.0 \mathrm{~mm}$ annular space provides the thermal insulation that reduces the heat transfer to the crucible and metal sample during freezing or melting experiments. The design also reduces the need for close control or knowledge of the furnace temperature in many operations.

Three Chromel-P/Alumel thermocouples (TC) enclosed in alumina sheaths control the furnace temperature. Two of the thermocouples (differential) are referenced to the temperature at the middle of the center core block; the measuring junction of one is located approximately $1.2 \mathrm{~cm}$ up into the top core block and that of the second is similarly located down into the bottom core block. The third TC is referenced outside the furnace and the measuring junction is located in the middle of the center block.

Each differential TC is connected in series with a stable voltage source (powered by mercury cells) which is adjustable between $\pm 75 \mu \mathrm{V}\left( \pm 1.5^{\circ} \mathrm{C}\right)$. The center block TC connects to a reference junction, which is self-compensating for changes in room temperature, and to a voltage source that has (i) a range of $15 \mathrm{mV}$, (ii) a stability of 0.01 percent, and (iii) a reproducibility of setting of better than $2 \mu \mathrm{V}$. The combined output of each TC and voltage source is amplified by a chopper type DC amplifier whose output operates a "three-mode" controller. The zero stability of the amplifier is better than $0.5 \mu \mathrm{V}$. The output of each controller is an adjustable linear (proportional) function of the input, its rate of change, and its time integral. The signal from the controller operates a gate drive for a fullwave silicon-controlled rectifier which, in turn, controls the power supplied to the heater. The rectifiers do not cause detectable interference with any other equipment (low level null detectors, a-c and d-c bridges, etc.) in the room. Each aluminum block of the core is grounded by means of a heavy gold wire.

The control of the furnace was made very flexible in that several combinations of manual and automatic controls may be selected for operating the furnace, including the provision for independently setting the temperature offset between the center and top and between the center and the bottom core blocks. A freeze is usually conducted with each of the three heaters under the automatic control of their corresponding TC's. A plot of thermometer resistances (relative to that at the center of the center block) as a function of depth in the core of the furnace, while the furnace and a tin-point cell was at $235^{\circ} \mathrm{C}$, is shown in figure 26 . These measurements were made with a small fourlead platinum resistance thermometer, $2.8 \mathrm{~mm}$ diameter and $2 \mathrm{~cm}$ long, having a nominal ice-point resistance of $50 \Omega$.

This furnace, which was designed and built at the NBS, is more sophisticated than necessary for calibrating thermometers at the tin and zinc fixed points with reasonable (better than $0.002{ }^{\circ} \mathrm{C}$ ) accuracy. It was designed for special studies of the freezing and melting phenomena of tin and zinc. Tin and zinc freezes for calibrating SPRT's have been performed very successfully in many laboratories with furnaces that contain a single copper block and employed a manually controlled heater. Such calibrations have been performed in furnaces that exhibit core temperature gradients of $1.5^{\circ} \mathrm{C}$ over the length of the crucible containing the sample [53] and relatively long response times. Realization of the tin and zinc freezing points with reasonable accuracy is principally dependent upon the high purity of freezing-point samples and the use of proper freezing and measurement techniques.

\subsection{Tin-point Cell}

The realization of either the tin-point or the steam-point is necessary for the calibration of SPRT's in accordance with the specifications set forth in the text of IPTS-68. The tin-point (231.9681 $\left.{ }^{\circ} \mathrm{C}\right)$ has two distinctive advantages; first, the temperature is much closer than the steam point to the midpoint between the triple point of water and the zinc point and, therefore, tends to produce less average error in the calibration of the thermometer (see fig. 17), second, and of greater practical importance, the solid-liquid equilibrium temperature of tin is 8600 times less sensitive to pressure changes than the liquid-vapor equilibrium temperature of water at 1 atm. For the freezing point of tin, $d t / d p=+4.3 \times 10^{-6}{ }^{\circ} \mathrm{C} /$ torr or $+2.2 \times 10^{-5}{ }^{\circ} \mathrm{C} /(\mathrm{cm}$ column of liquid tin $)$. Therefore. knowledge of the pressure within \pm 1 torr is adequate for determining the temperature of a tin-point cell.

The tin sample for the freezing-point cell must be of high purity (nominally $>99.999 \%$ ) and the freezing apparatus must be designed and operated to interpose a solid-liquid interface of tin completely around the resistance element and lower part of the thermometer stem. The immersion of the resistor must be sufficient to prevent sensible heat flow from the resistor up the stem of the thermometer. In addition, the sample holder must be chemically inert and not introduce any impurities that would affect the freezing temperature of tin. At NBS the tin sample is contained in a closed crucible of high-purity graphite. A re-entrant well, 


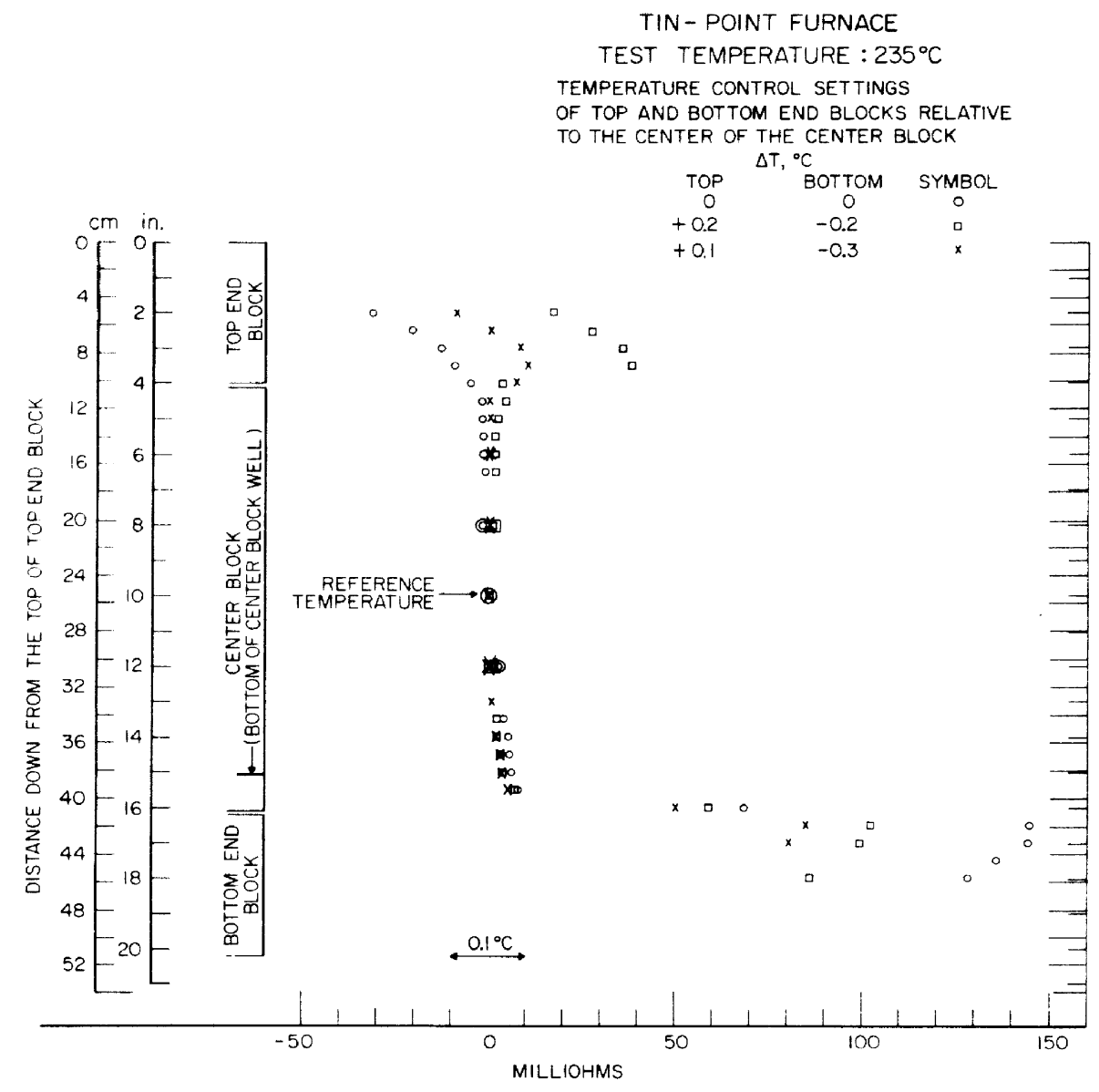

FIGURE 26. Vertical temperature profile of tin-point furnace at $235^{\circ} \mathrm{C}$.

The vertical distances are relative to the top of the top core block; the thermometer resistances are relative to that observed at $25.4 \mathrm{~cm}$ from the top of the top core block (close to the center of the center core block). The resist. ance of the thermometer at the reference temperature was about $95 \Omega$. The resistance thermometer $2.8 \mathrm{~mm}$ diameter $\times 2.0 \mathrm{~cm}$ long) sensed the average temperature of a section of well about $2 \mathrm{~cm}$ long.

also of high-purity graphite, is screwed into the lid The assembly is shown schematically in figure 27 . The cell assembly permits sealing the stem of the thermometer, as well as the crucible with its charge of tin, in helium gas which provides an inert atmosphere for both the crucible and the tin and additionally improves the thermal conductance to the thermometer.

Because of the relatively high thermal impedance of gas, even helium, considerable effort was made to obtain small clearances at the heat shunts to keep the thermal impedance as low as possible. This is practical with components of borosilicate glass and graphite because their thermal coefficients of expansion are similar. The outer borosilicate glass cell was formed from precision bore tubing and its external diameter was ground to fit the furnace sleeve (see $(\mathrm{H})$ fig. 25). The two graphite heat shunts $(G)$, shown in figure 27 , were fitted closely to both the thermometer guide tube $(F)$ and the glass cell $(\mathrm{H})$. The shunts primarily serve to conduct heat to the inner glass tube of the tin-point cell, thereby improving the "immersion characteristics" of the thermometer. (See the end of this section for results of tests on the immersion characteristics of a SPRT in the tin-point cell.) The space between the heat shunts is loosely filled with high purity Fiberfrax (washed) insulation to eliminate convection currents and radiation losses from the top of the crucible and the heat shunts; it also vertically positions the heat shunts.

The equipment arrangement for filling the crucibles is shown in figure 28. The bell jar (which contains the metal sample and its funnel and crucible) is evacuated to about 0.01 torr by a mechanical pump that is "trapped" with a molecular sieve and the temperature of the enclosed assembly is then raised to a few degrees below the tin point. After 1 hour, the induction heater power is increased so that the tin sample will melt and flow into the crucible within about $15 \mathrm{~min}$. Next, the sample is allowed to cool nearly to room temperature, the crucible is removed from the bell jar, and the crucible is slipped into the special borosilicate glass cell, figure 29. The glass cell is then purged with helium, lowered into the furnace that is used for realizing the freezing point, and the tin reheated to about $250{ }^{\circ} \mathrm{C}$. Subsequently, the glass cell is 


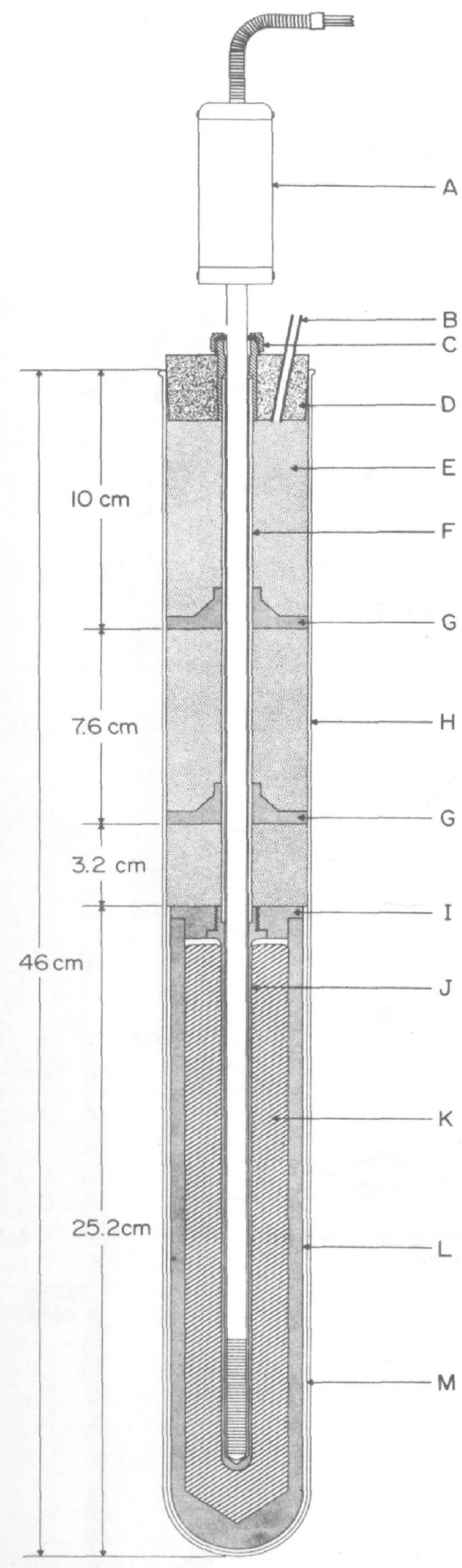

Figure 27. Metal freezing-point cell. A. Platinum resistance thermometer.

B. To helium gas supply and pressure gauge. C. Thermometer stem seal with silicone rubber. D. Silicone rubber cap.

E. Insulation, washed Fiberfrax. raised out of the furnace and the graphite lid and well assembly are smoothly and quickly pushed into place. To perform the assembly easily the outside diameters of the crucible and lid were made equal and the inside dimensions of the glass tube only slightly (perhaps $0.01 \mathrm{~mm}$ ) larger. The glass tube served to guide and correctly position the lid to cover the crucible. The lid is closely fitted to the crucible to minimize the possibility of later contamination. Finally, the crucible is again allowed to cool, removed from the special glass cell, and assembled as a freezing-point cell such as is shown in figure 27.

In the preparation for tin freezing point measurements, the tin-point cell is placed in the furnace (held about $10{ }^{\circ} \mathrm{C}$ above the tin point) until complete melting occurs. During the heating process the cell temperature, which is monitored with a SPRT, rises until melting begins, becomes nearly constant until the melting is completed, and then rises again. There is no reason to raise the temperature of the tin more than a few tenths of a degree above the melting point. In a furnace with large temperature gradients precautions should be taken to make certain that the metal is completely melted. There is no evidence to indicate that heating the tin to several degrees above its melting point in an inert atmosphere is harmful to the sample.

After the melting is completed, the furnace is allowed to cool to the tin point. A thermometer is inserted in the cell well and instrumented so that a temperature range of $25{ }^{\circ} \mathrm{C}$ below the tin point can be visually monitored within about $0.5^{\circ} \mathrm{C}$ by a person, standing beside the furnace, holding a tin cell ready to be reinserted. A galvanometer is used for this purpose. When the cell has cooled to the tin point it is removed from the furnace. The cell temperature, after a few seconds, will suddenly decrease very rapidly, perhaps as much as 20 or $25{ }^{\circ} \mathrm{C}$. As soon as its temperature ceases to drop, the cell is immediately lowered into the furnace again; subsequently, the cell temperature will increase even more rapidly than it previously decreased. If the cell temperature is examined in more detail, the final approach to the tin point (the last few ten-thousandths of a degree) will be seen to take a few minutes (see fig. 30). When the temperature plateau is reached, the SPRT is measured. It may then be removed and a second SPRT inserted. The second thermometer is preheated above the tin point so that its temperature, during insertion into the tin point cell, slightly exceeds that of the cell. Figure 31 shows the results of successive insertions of the same SPRT in a single tin freeze.

F. Thermometer guide tube, borosilicate glass.

G. Heat shunt, graphite.

H. Borosilicate glass cell.

I. Graphite cap (lid).

J. Graphite thermometer well.

K. Metal sample.

L. Graphite crucible.

M. Insulation, Fiberfrax paper. 


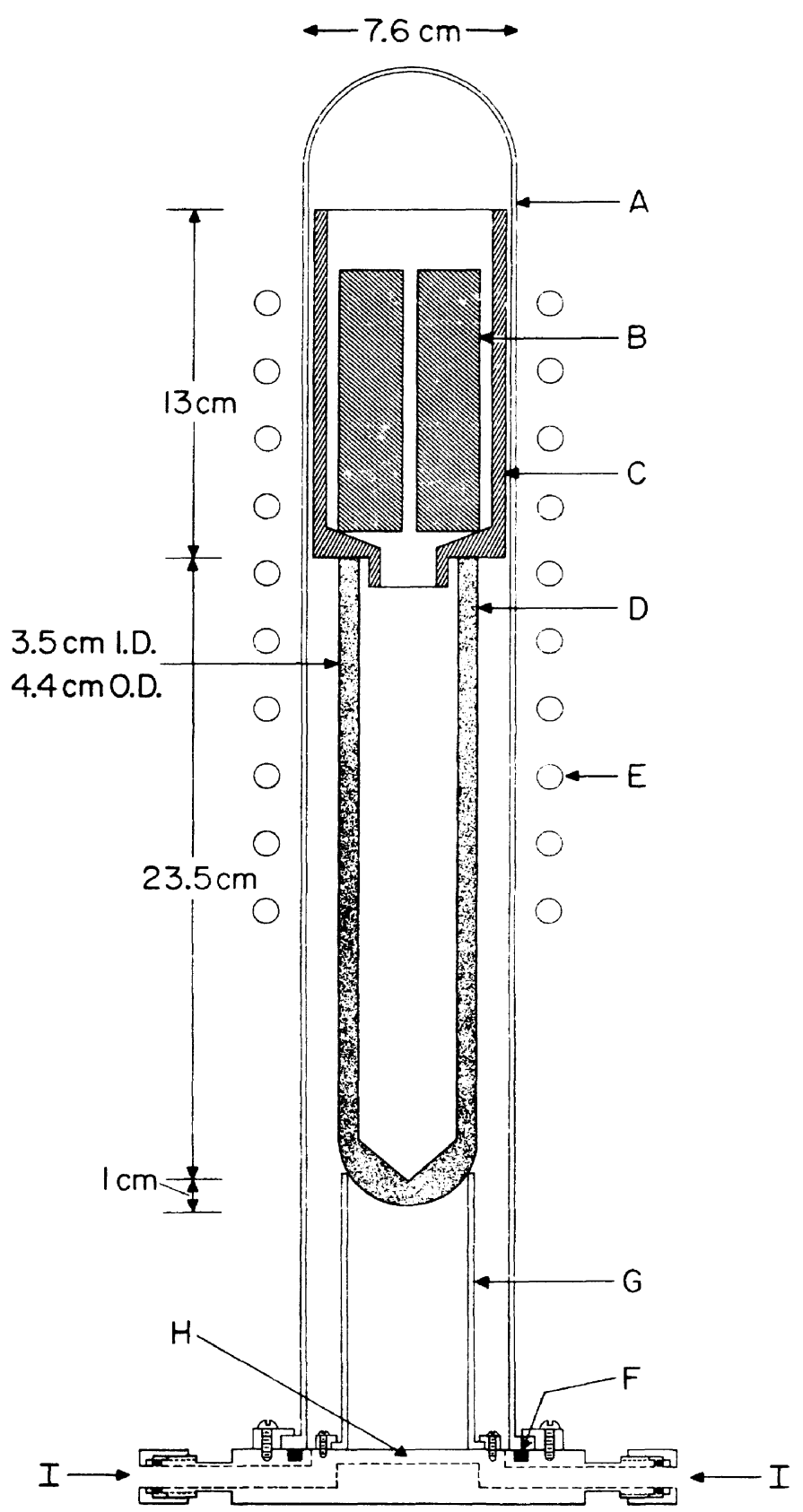

FIGURE 28. Arrangement for filling the freezing-point cell with

metal sam
A. Borosilicate glass envelope.

B. Metal sample.

C. Graphite sample holder and funnel.

D. Graphite crucible.

E. Induction heater coils.

F. " $D$ " ring groove.

G. Borosilicate glass stand.

H. Slot for pumping out and gas purging

I. Connection to vacuum and purified argon supply. 


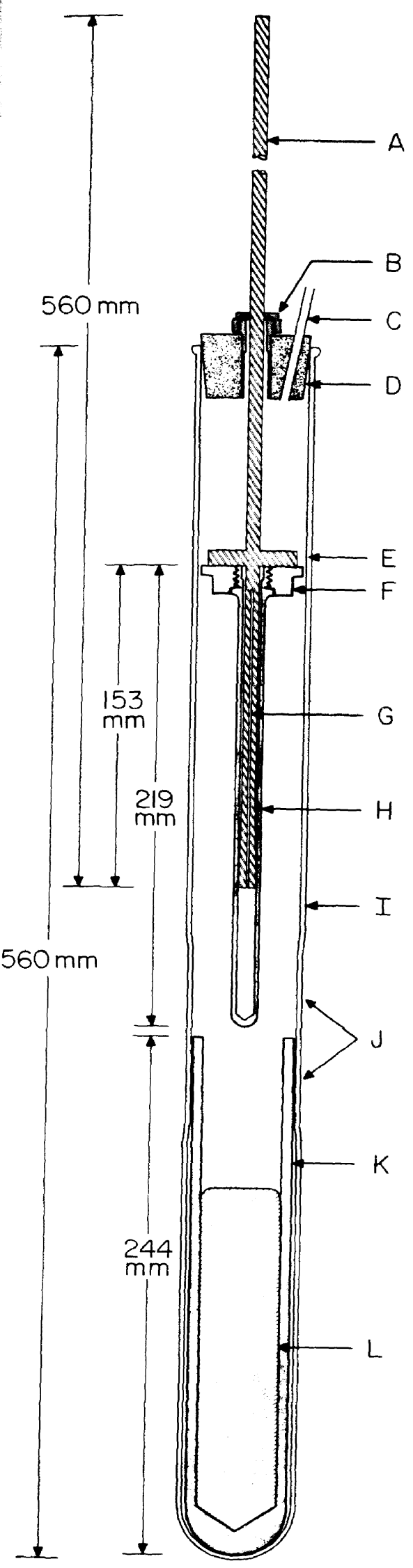
A. Stainless steel pusher rod.
B. Gas seal with silicone rubber. Permits linear motion of the pusher rod (A).
C. Inlet for purified helium gas that is used in purging and maintaining positive pres - sure of the gas during assembly.
D. Silicone rubber cap.
E. Stainless steel flange attached to the pusher rod for pressing against the graphite lid during assembly.
Fraphite lid for the metal sample cell
F. Graphite lid for the metal sample cell.
G. Slit on the pusher rod. The two halves are sprung out to hold up the graphite 列
H. Graphite thermometer well
1. Borosilicate glass tube.
J. Section of glass tube shrunk to fit the crucible and bid so that lid can be easily guided onto the crucible.
K. Graphite crucible.
L. Molten metal sample.

FIGURE 29. Apparatus and method for installing graphite thermometer well and lid in the graphite crucible containing the molten, metal freezing-point sample. 


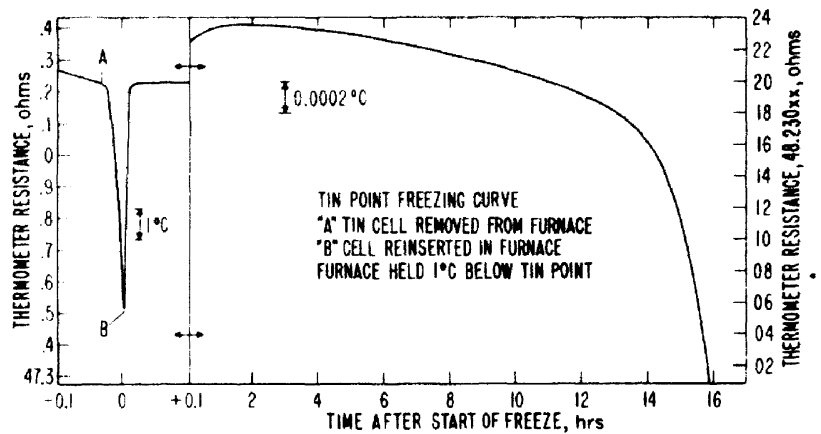

FIGURE 30. The freezing curve of tin obtained using an a-c bridge with the head of the SPRT adapted for coaxial connectors.

The furnace was controlled to be $0.9 \mathrm{~K}$ below the tin point. The mass of tin sample was 1300 k.

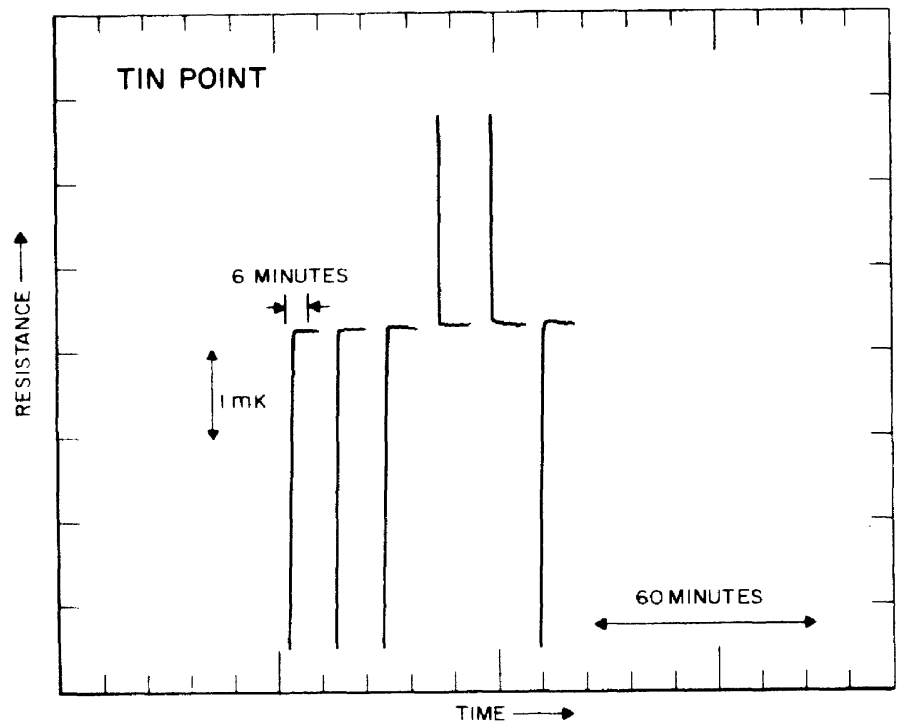

Figure 31. Consecutive measurements of the resistance of a SPRT in the same tin freeze.

(An a-c bridge was employed in the measurements.)

Following each equilibrium resistance observation, the SPRT was completely withdrawn from the tin-point cell, preheated close to the tin point in an auxiliary furnace,

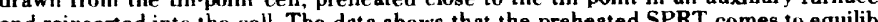
and reinserted into he cell. The data shows that the prehealed SPRT comes to equilib num in a very shor time. In two of the cases shown, the temperature of the SPRT when
inserted into the cell was slightly above the tin point. (For comparison when the SPRT was not preheated close to the cell temperature, see fig. 35 .)

An experimental procedure similar to that described above for observing the supercooling of the tin may be used to check the preheating of the SPRT before inserting it into the tin-point cell. The thermometer should be preheated and held in the air over the tin cell while monitoring the decreasing thermometer temperature. As a first approximation, the thermometer can be inserted into the tin-point cell when its indicated temperature is $30{ }^{\circ} \mathrm{C}$ above the tin point. If subsequent close monitoring indicates that the thermometer temperature is rising, then the thermometer was too cold when it was inserted. Several thermometers may be consecutively calibrated during one freeze if each is properly preheated (see fig. 31). A simple check

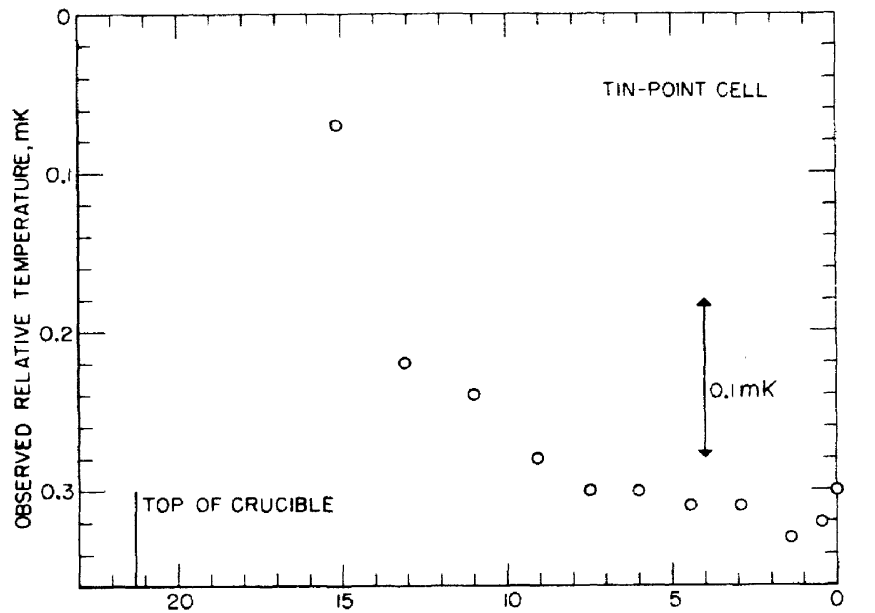

DISTANCE OF THERMOMETER TIP ABOVE BOTTOM OF THERMOMETER WELL, $\mathrm{cm}$

FIgURE 32. Immersion characteristic of a SPRT in a tin-point cell.

The data show that the indications of the SPRT are very nearly the same between The data show that the indications of the SPRT are very nearly the sam
about 8 or $10 \mathrm{~cm}$ above the bottom and the bottom of the thermometer well.

on the temperature constancy during a freeze is afforded by employing the same thermometer in both the first and last measurement in a series of SPRT calibrations. At NBS a single SPRT is set aside and only used for this purpose as an experimental control.

The immersion characteristics of a SPRT were tested in one of the tin-point cells that were described earlier. The observed resistances are shown in figure 32 as a function of the distance from the bottom of the crucible thermometer well. The results show that the immersion of the SPRT in the cell is more than adequate. This figure does not, however, show the temperature gradient expected from the change in pressure with depth. ${ }^{4}$

In the reduction of the calibration data the temperature at the point of immersion of the SPRT in the tin-point cell is employed. The value of temperature assigned in the text of the IPTS-68 is adjusted for the departure from the equilibrium conditions specified for the fixed point; i.e., an adjustment of temperature is made for the effect of the hydrostatic head of liquid tin and for any departure from 1 standard atm of the gas pressure in the cell.

The high reproducibility of the tin point may be seen in the results of tests (made at the NBS) that compare the freezing points of a number of tin samples. The average standard deviation of measurements on a given cell is $\pm 0.05 \mathrm{mK}$ from freeze to freeze (see fig. 33). All of the measurements are plotted relative to the mean of samples $6 \mathrm{C}$ and $6 \mathrm{E}$. The individual measurements (one for each freeze) are shown as short horizontal bars; four freezes are represented for each cell with the exception of $6 \mathrm{~K}$

4 Recent work done at NBS indicates that the temperature gradient expected from the change in pressure with depth is distorted or obscured when observations are made from within the graphite thermometer well if the solid liquid interface is not very close to and surrounding the well. Further work in this area is in progress. 
on which only two freezes were performed. The values are based on the determinations of $R(t) / R(0)$ that were obtained from extrapolations to zero thermometer current of observations of $R(t)$ 's and $R(0)$ 's at 1 and $2 \mathrm{~mA}$. The $R(t)$ 's were measured 1.5 hours after the initiation of a freeze. The $R(0)$ 's were measured immediately after every determination of $R(t)$ 's. The samples identified as 5 were reported to be 99.999 percent pure [35]; the samples identified as 6 were reported to be 99.9999 percent pure [35]. The sample identified $6 \mathrm{M}$ was accidentally overheated to a temperature above $500^{\circ} \mathrm{C}$.

\subsection{Zinc-Point Cell}

The equipment and procedures used at NBS for filling graphite crucibles with zinc are identical to those employed for tin, except that after evacuation at a temperature slightly below the tin point, an atmosphere of purified dry argon is admitted into the bell jar. Because of the relatively high vapor pressure of zinc at the melting point (over 0.1 torr), filling the graphite crucible by melting the sample in vacuum is less practical. The power of the induction heater is raised sufficiently to melt the zinc sample (approximately $1280 \mathrm{~g}$ ) in about $30 \mathrm{~min}$. In spite of the presence of argon gas, enough zinc vapor diffuses and is deposited on the surface of the bell jar to cause difficulty in observing when the zinc is completely melted. The sample is allowed to cool and the assembly of the zinc-point cell is carried out in the same manner as the tin sample (see sec. 7.4).

To realize the freezing point of zinc with an accuracy of about $\pm 0.002{ }^{\circ} \mathrm{C}$ the furnace need not be very complex. However, a zinc sample of high purity and the use of proper freezing-point techniques are important. The zinc is melted in the furnace while being monitored with a SPRT. The experimental procedure for realizing the freezing-point of zinc differs appreciably from that employed for tin. The zinc freeze is initiated in the furnace, unlike the tin freeze where the cell is removed from the furnace to initiate the freeze. The furnace temperature is reduced so that the

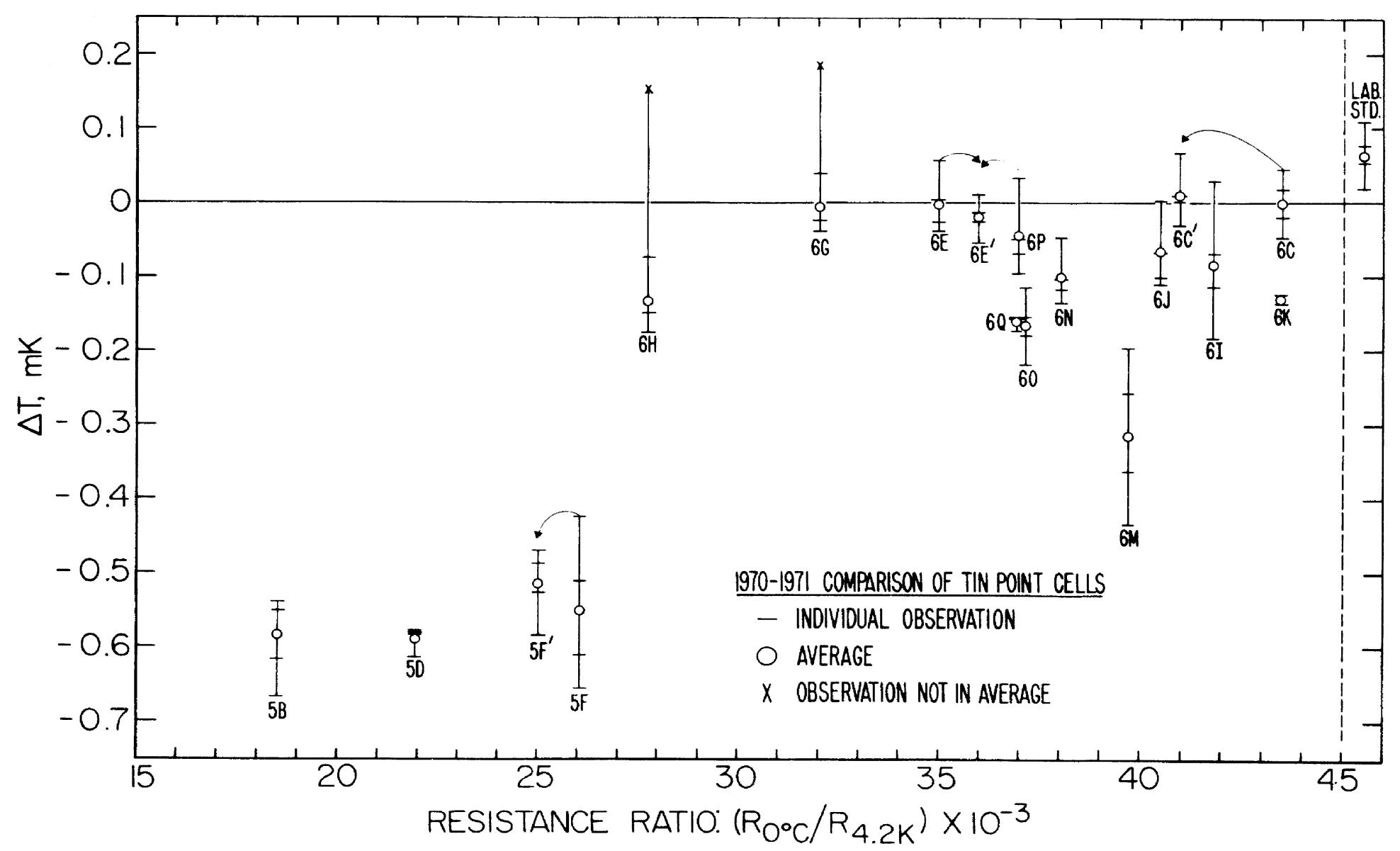

FIGURE 33. Comparison of tin-point freezing plateau temperatures with resistance ratios: $\mathbf{R}\left(0^{\circ} \mathrm{C}\right) / \mathbf{R}(4.2 \mathrm{~K})$.

The prefixes 5 and 6 indicate samples nominally 99.999 percent pure (Lot No. 6637) and samples nominally 99.999 percent pure (Lot No. 6779), respectively. The alphabetic character identifies the cell. The prime (') identifes a second set of me asurements on the same cell. LAB. STD. is a tin-point cell that has been used for over four years at the NBS in the calibration of SPRT's. Observations from four separate freezes are shown for each cell The plateau temperat ure selected was the reading 1.5 hours after the initiation of the freeze the temperat ure corresponded to about 25 percent frozen. The temperatures of the SPRT during the previous hour differed typically 0.00002 or $0.00003 \mathrm{~K}$ but never more than 0.0001 $K$ from the selected temperatures of any measurements. The observed plateau temperatures are shown relative to the mean value obtained for the tin-point cells $6 \mathrm{C}$ and $6 \mathrm{E}$. $\mathrm{C}$. $6 \mathrm{M}$ was accidentally overheated above $500^{\circ} \mathrm{C}$ before the measurements. Only two measurements were obtained for cell $6 \mathrm{~K}$. (Resistance ratio measurements were made by $\mathrm{R}$. $\mathrm{L}$. Powell at the Boulder Laboratories of the National Bureau of Standards.) 
sample temperature, as measured within the well, reaches the zinc-point temperature at a cooling rate of 0.1 to $0.2{ }^{\circ} \mathrm{C}$ per minute. The zinc supercools below the zinc point by 0.02 to $0.06{ }^{\circ} \mathrm{C}$ (as measured by the SPRT) before the recalescence occurs. The subsequent rise toward the plateau temperature, while rapid at first, becomes very slow after several minutes (see fig. 34). To hasten this process the induced-freeze suggested by McLaren [32] is employed to form a second solid-liquid interface immediately adjacent to the well. (The first solidliquid interface occurs at the crucible wall.) After the zinc cell has completed the initial rapid freeze following the supercool described above, two cold thermometers are inserted into the well to freeze the zinc around the well; a solid-liquid interface that completely surrounds the well is necessary and a single thermometer may not freeze a complete coating of zinc on the well. The resistance of the second thermometer will rise to a steady value (within $10^{-4}{ }^{\circ} \mathrm{C}$ ) within a few minutes (typically 10 to $15 \mathrm{~min}$ ) after insertion and will remain nearly constant for a period of time that is determined by the freezing rate of the outer solid zinc layer and the purity of the zinc. (The rate of freezing, of course, depends principally upon the heat loss to the furnace, i.e., the temperature setting of the furnace). Freezes lasting as long as three days have been observed at NBS, but 12 to 16 hour freezes are more typical. As with tin, SPRT's may be consecutively calibrated in a single zinc freeze if the thermometers are preheated (see fig. 35). The preheating of the thermometer serves to minimize the growth of the inner freeze on the well and thereby extend the useful life of the freeze. Care must be exercised to avoid melting a hole in the inner freeze or loosening the mantle on the well so that it slides to the bottom of the crucible. To ensure that no melting and no excessive freezing occurs, the thermometer is preheated to a temperature slightly below the zinc point before inserting it into the cell. As with tin, a SPRT is set aside at NBS for checking the constancy of the freeze, and it is used for both the first and last measurements in each freeze. The freezing-point measurements are normally not conducted at exactly the specified pressure of 1 standard atmosphere and a correction is made for this departure. The pressure at the interface surrounding the thermometer resistor includes both the gas pressure over the sample and the pressure due to the head of liquid zinc. For zinc at the melting point the value of $d t / d p$ is $+5.7 \times 10^{-6}$ ${ }^{\circ} \mathrm{C} /$ torr or $+2.74 \times 10^{-5}{ }^{\circ} \mathrm{C} / \mathrm{cm}$ column of liquid zinc [32].

The immersion characteristics of a SPRT were tested in one of the zinc-point cells that were described. The observed resistances are shown in figure 36 as a function of the distance from the bottom of the crucible thermometer well. The results show that the immersion of the SPRT in the cell is more than adequate.

\subsection{Oxygen Normal Boiling Point}

The oxygen normal boiling point calibration is realized at the NBS by reference to the NBS-55 scale adjusted to correspond to the IPTS- 68 (see sec. 7.7). The temperature (hotness) assigned to the oxygen point, now maintained by SPRT standards, is $0.0019 \mathrm{~K}$ lower [8] than that maintained previously on the NBS- 1955 scale [50]. This change resulted from efforts made to achieve uniformity

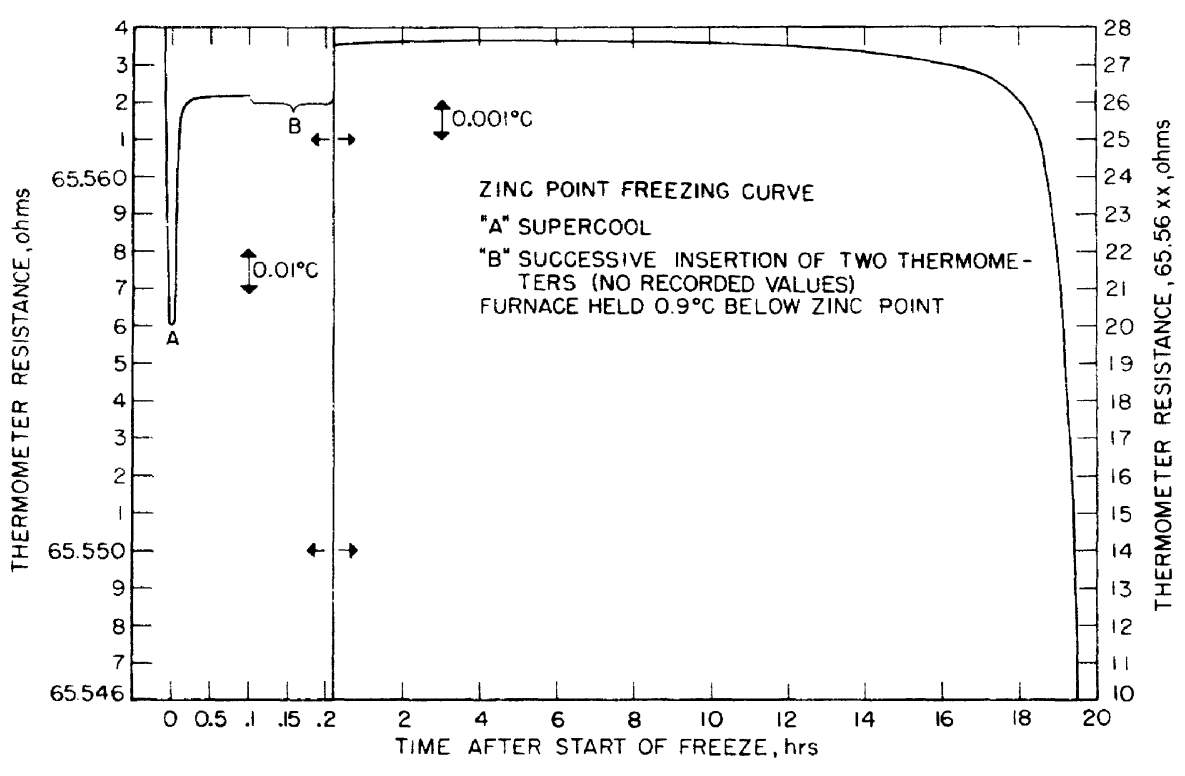

FIGURE 34. The freezing curve of zinc obtained using an a-c bridge. (The head of the SPRT had been adapted for coaxial connectors.)

The furnace was controlled at $0.9 \mathrm{~K}$ below the zinc point. The mass of zinc sample was $1280 \mathrm{~g}$. Two cold SPRT's were inserted in the cell at " $B$ " to freeze a coating of zinc around the graphite thermometer well. 


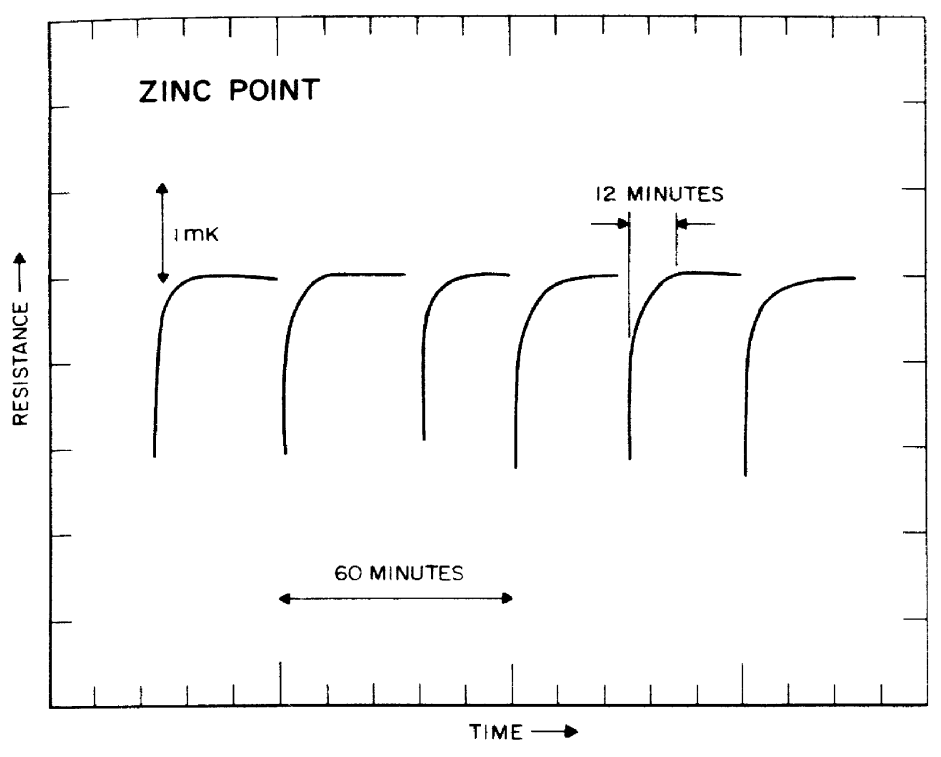

FIGURE 35. Consecutive measurements of the resistance of a $S P R T$ in the same zinc freeze.

(An a-c bridge was employed in the measurements.)

Following each equilibrium resist ance observation, the SPRT was completely with drawn from the zinc-point cell, exposed to the ambient temperat ure for one minute. drawn rom the zinc-point cell, exposed to the ambient temperature for one minute, and reinserted into the cell. In the actual procedure employed in calibration the SPRT is preheated close to the zinc point (see text). The dat a show that the SPRT comes rapidly to equilibrium even when inserted into
comparison when the SPRT was preheated, see fig. 31.)

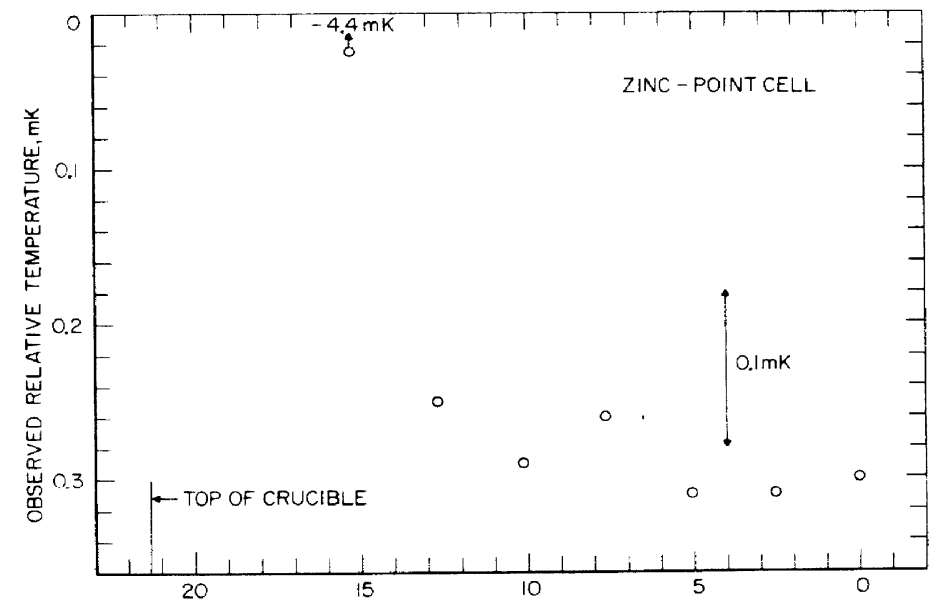

DISTANCE OF THERMOMETER TIP ABOVE BOTTOM OF THERMOMETER WELL, $\mathrm{CM}$

FIGURE 36. Immersion characteristics of a SPRT in a zinc-point cell.

The data show that the indications of the SPRT are very nearly the same between about 8 or $10 \mathrm{~cm}$ above the bottom and the bottom of the thermometer well.

in several national temperature scales; for details see reference [8]. Calibrations near the oxygen point are performed by inserting the SPRT's to be calibrated and an SPRT standard in the apparatus shown in figure 37 . In the preparation for calibration the apparatus is evacuated and immersed in liquid nitrogen to the level indicated. There are eight thin-walled Monel wells (A) extending into the copper block (P). Two of these wells are one-half inch in diameter to accommodate capsule type thermometers in holders. Thermometers are sealed

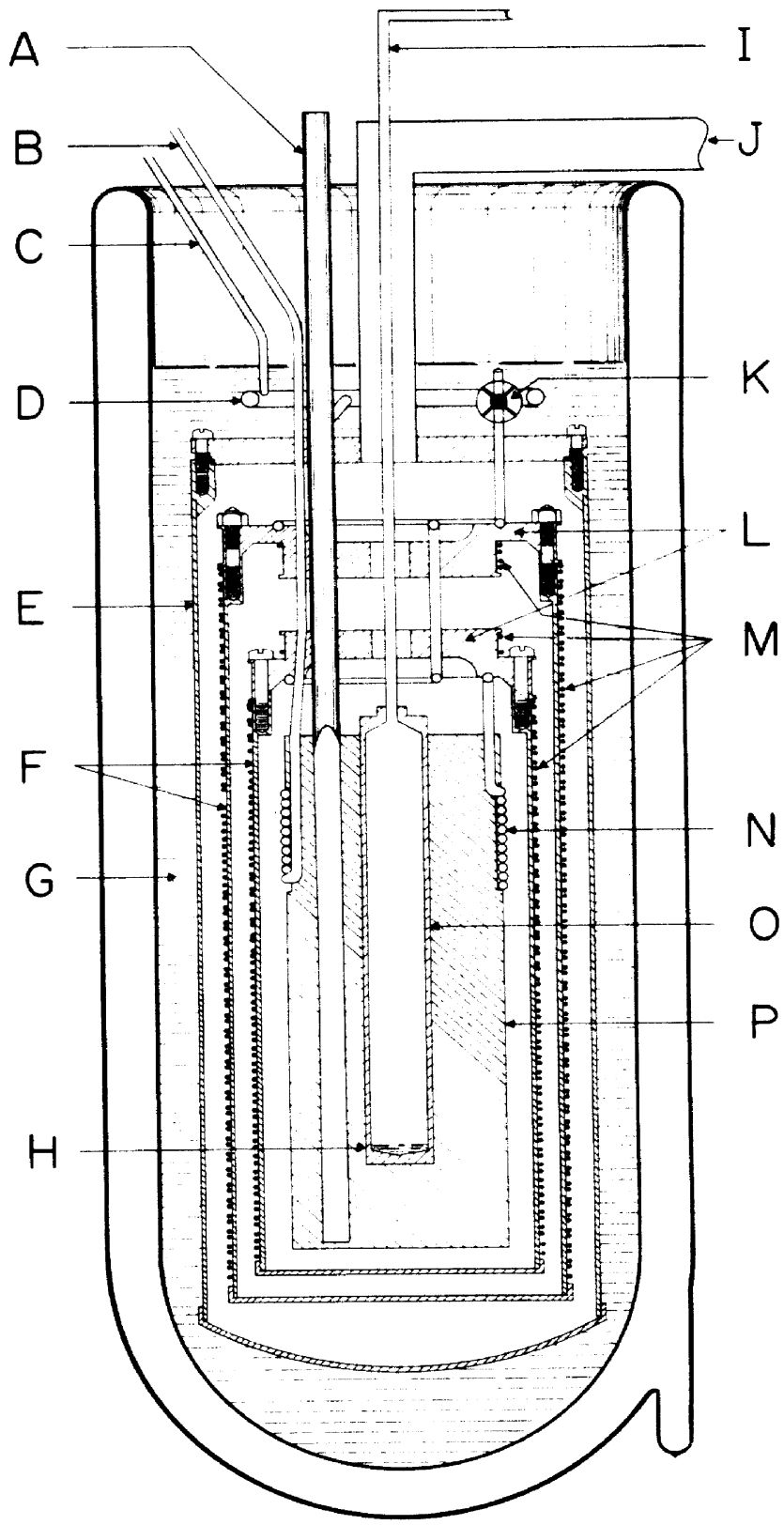

FIGURE 37. Cryostat for comparison calibration of thermometers with standards at the axygen normal boiling point.

A. Thermometer well; eight wells are located around the copper block. The SPRT's are sealed at the top with molded silicone rubber.

B. Tube to vacuum pump to draw liquid nitrogen through cooling tubes.

C. Tube to helium gas supply.

D. Manifold for distributing helium gas to the thermometer wells.

E. Envelope (brass).

F. Radiation shields (copper)

(;. Liquid nitrogen.

H. Liquid oxygen. (Not employed in comparison calibrations.)

1. Vapor-pressure tube, to differential pressure diaphragm and manometer.

J. High vacuum line

K. Valve to control the liquid nitrogen input for cooling.

L. Top heat shields, to control thermometer well and thermometer stem temperatures. M. Heaters.

N. Cooling tubes (thin-wall Monel.

O. Oxygen bulb.

P. Copper block.

into the wells at the top by a molded band of very soft silicone rubber. The thermometer wells are filled with helium gas to a pressure that is slightly above atmospheric. The helium enhances the thermal contact between the wall of the well and 

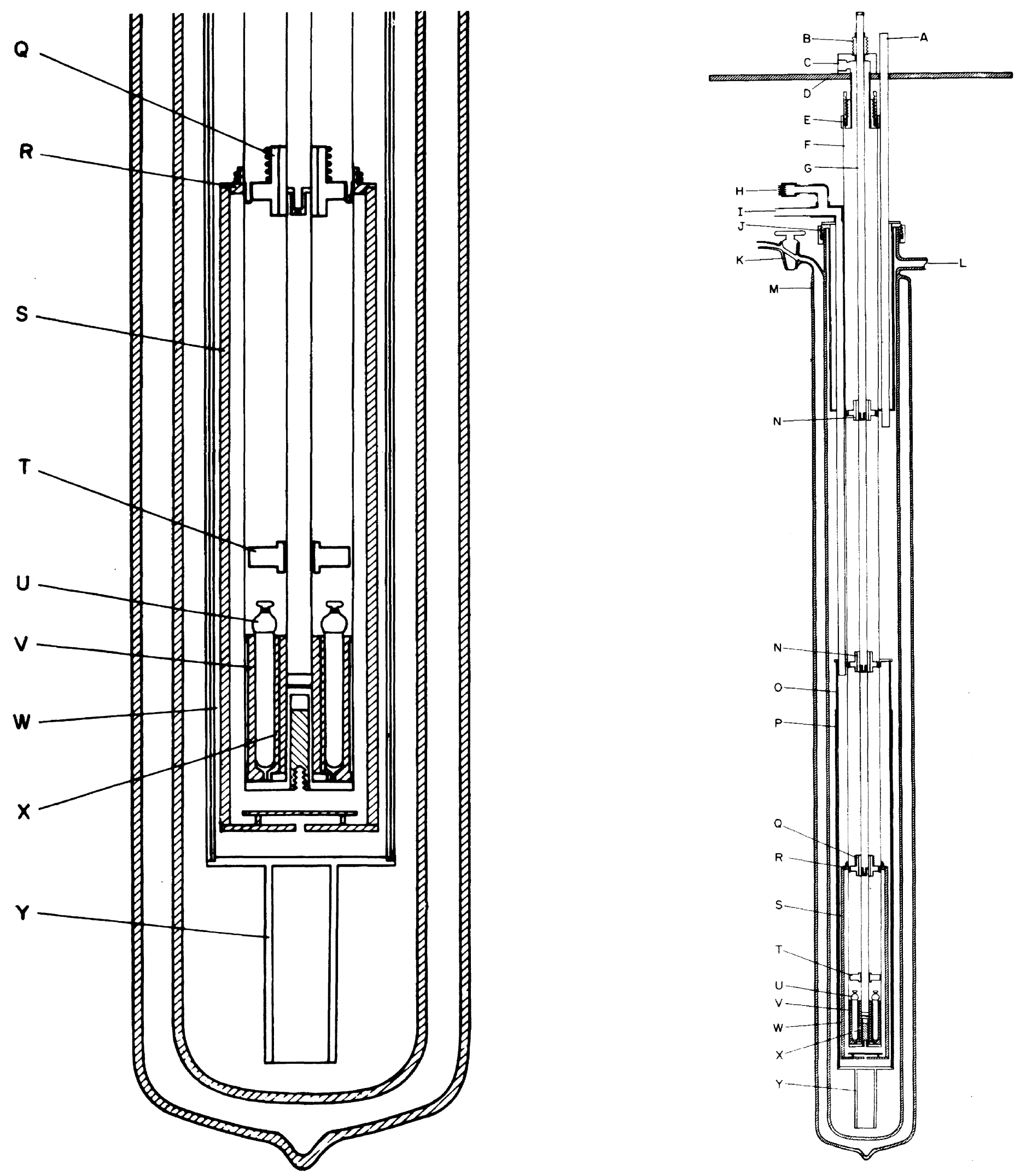

FIGURE 38. Cryostat for the intercomparison of capsule type SPRT's. 
the thermometer and prevents condensible gases from entering the wells. The copper block $(P)$ and shields ( $F$ and $L$ ) are maintained under vacuum. They are initially cooled by admitting nitrogen gas and/or liquid through the valve $(K)$ shown just below the surface of the liquid nitrogen. The cool vapor flows through coils $(\mathrm{N})$ on the top shields and coils on the massive center block, finally passing through (B) to a large-capacity vacuum pump. After cooling the block to a temperature near the oxygen point, the valve is closed and the nitrogen gas in the cooling coils is removed; the temperature of the block is brought within $1 \mathrm{~K}$ of the oxygen point, and the outer shields are controlled at the temperature of the block. Experience shows that the temperature of the block can be maintained more nearly constant by allowing the inner shields to "float" without heating at a temperature near that of the block. [Heaters (M) on the copper block and the inner shields are used, when desired, to attain higher temperatures]. The outer shields are controlled to be at the temperature of the block by means of one differential thermopile between the top shield and the block, and a second thermopile between the outer top shield and the outer side shield. The signals from these thermopiles go to commercial low level chopper type $d$-c amplifiers and thence to three-mode controllers; the output signals from the controllers are then raised to power levels that are sufficient to supply the shield heater (less than $1 \not W$ ). The measurements on the thermometer to be calibrated and the standard thermometer are made simultaneously using two Mueller bridges. The standard deviation of the intercomparison is less than the equivalent of $0.1 \mathrm{mK}$.

\subsection{Comparison Calibration Between 13.81 and $90.188 \mathrm{~K}$}

To determine temperatures below the oxygen normal boiling point, the text of IPTS-68 (Appendix A) assigns values to defining fixed points and prescribes the form of interpolation formulae. At the time the IPTS -68 was adopted no national laboratory could satisfactorily realize all the fixed points. The four temperature scales (e.g., NBS-55) upon which the extension of the IPTS -68 below the oxygen point was principally based are each highly reproducible. The text of the IPTS-68, therefore, formally recognized that the use of these national scales, adjusted by the published differences [8], will give a close approximation to the IPTS-68 below $90.188 \mathrm{~K}$. At NBS a reference group of capsule type SPRT's maintains the "national scale" (NBS55 ). The NBS version of IPTS-68 (NBS-IPTS-68) is achieved by making reference to the NBS-55 scale and utilizing the published differences between the NBS -55 scale and the IPTS -68 referred to above. Thermometers are calibrated by intercomparison in a nearly isothermal copper block with one of the thermometers of this reference group. A second thermometer from the reference group is also included in the comparison to serve as a check on both the reference thermometer $b$ eing used and the comparison techniques. These two thermometers will be referred to, henceforth, as the first and second standards, respectively.

The comparison of capsule type SPRT's is performed in a cryostat that has been built and operated rather like an adiabatic calorimeter. The apparatus is shown in figure 38. The copper comparison block (V), shown enlarged in figure 39 , has wells for six thermometers, two standards and four thermometers under test. The schematic in figure 40 shows that the first standard is wired independently of the other five thermometers. The wiring arrangement employed for the five thermometers requires only $2 n+2$ leads for $n$ thermometers, in this case, 12 leads, thus minimizing the number of leads and, therefore, the heat transfer through the leads with the sur oundings. The first standard is electrically isolated from the others. This permits the use of two bridges to make simultaneous measurements on the first standard and any one of the thermometers to be calibrated or the second standard. The resistance measurements are made with two Mueller bridges.

A. Guide for directing transfer tube into liquid helium Dewar.

B. Metal bellows to permit differential expansion between the central tube and the supporting thermometer block well.

C. Exit for the vacuum line and electrical leads to the thermometers, the thermocouples, and the heater on the copper thermometer block.

D. Supporting shelf.

E. Demountable "O" ring seai to the well around the thermometer block

F. Well around the thermometer block.

G. Central tube for supporting the thermometer biock

H. Seal for the electrical leads from the vacuum can that surrounds the shield and lower portion of the thermometer block well.

I. Line from vacuum can " $O$ ".

J. Demountable "O" ring seal to the liquid helium Dewar.

K. Glass stopcock to permit reevacuation of liquid helium Dewar.

L. Line for pumping the space within the liquid helium Dewar.

M. Liquid helium Dewar.

N. Thermal tie-down for lead wires.

o. Vacuum can that surrounds the lower portion of the thermometer block well and the thermal shieid.

P. Copper sleeve on vacuum can to maintain uniform temperature when bquid helium level is low.

Q. Thermal tie-down for leads similar to $\mathbf{N}$ with a heater and five-junction Chromel-P/constantan thermocouple for temperature control.

R. Copper ring on the thermometer block well with temperature control components similar to $Q$.

S. Heavy copper shield with temperature control components similar to $Q$.

T. Thermometer lead terminal block of anodized aluminum.

U. Capsule type platinum resistance thermometers thermally attached to copper block with vacuum grease.

V. Copper thermometer block with holes for six thermometers.

W. Location of thermocouple junctions placed on shield.

$X$. Reentrant "thumb" in the bottom of well. "Thumb" contains reference junctions for thermocouples on $R$ and $S$

Y. Heavy copper tail on vacuum can to reach liquid helium at a low level 


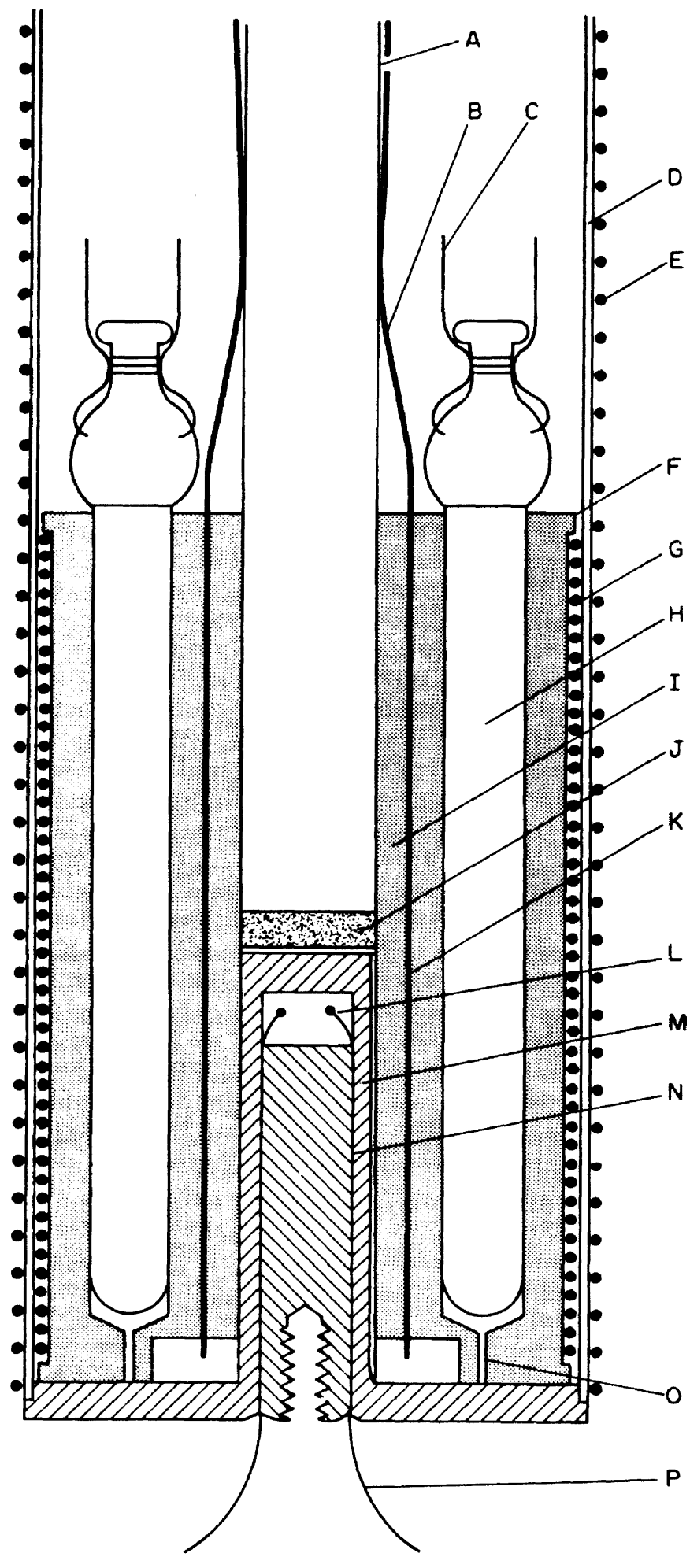

FIGURE 39. Thermometer comparison block.

A. St ainless steel tube t1. support thermometer comparison block.

B. Manganin leads for thermometers and copper leads for block he ater

C. Platinum leads of resistance thermometer.

D. Stainless steel well for thermometer bleck.

E. Heater distributed along well " $D$ ".

F. Copper thermometer block with wells for six thermometers.

G. Heater for thermometer block.

H. Platinum resist ance thermometer.

1. Copper sleeve (att ached to central supporting lube) with longitudinal grooves to serve as thermal tie-downs for manganin leads and thermocouples.

J. Brass plug to seal end of central suppert tube.

K. Location of grooves in steeve "I."

K. Location of gruoves in sleeve "l."
L. Reference junctions for thermocouples on shield (at "W" in fig. 38) and thermom eter block well ring heater (at " $R$ " in fig. 38).

M. Closely fitted reentrant copper "thumb" (with a groove for venting gas).

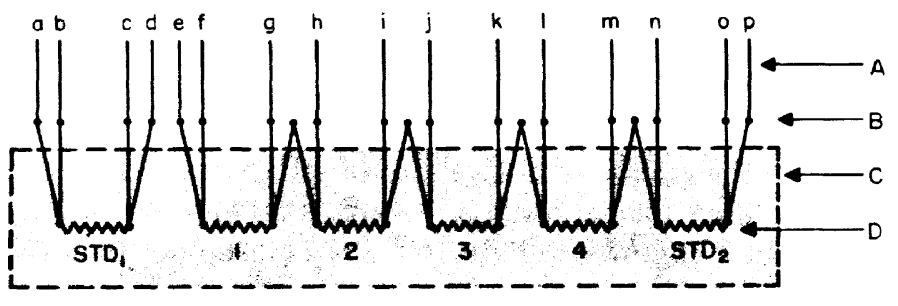

Figure 40. Schematic of wiring capsule-type platinum resistance thermometers in the copper comparison block of the cryostat for calibration.

To avoid excessive heating of the copper block, the bridpe connections to the thermometers, wired in series, are made as follows:

\begin{tabular}{c|c}
\hline SPRT & Leads used \\
\hline 1 & efgh \\
2 & ghij \\
3 & ijkl \\
4 & klmn \\
$\mathrm{STD}_{2}$ & mnop \\
\hline
\end{tabular}

A. Cryostat leads.

B. Thermometer connections made among them and with the cryostat leads.

C. Schematic of the copper comparison block.

D. Platinum resistance thermometers. STD 1 and $\mathrm{STD}_{2}$ are reference standards; others are to be calibrated.

To minimize the possible change in the difference of resistance in the potential leads, the leads from the thermometers that pass up through the cryostat are of No. 26 AWG (0.404 $\mathrm{mm}$ diameter) manganin wire insulated with a heavy coating of Formvar. The choice represents a compromise among the requirements of low heat conductivity, the resistance seen by the galvanometer, and lead-resistance stability. The leads are "tempered" by being placed in good thermal contact with the series of "thermal tie downs" (N) shown in figure 4l. The manganin leads and copper leads for thermocouples and heaters exit through a hard wax seal at $(C)$ the top of the cryostat and then join to heavier copper leads. The external copper leads are thermally insulated to minimize both the temperature change of the leads and any possible gradient change between leads. The resistances of the copper sections of the leads and those of the manganin sections of the leads were separately adjusted for common equality to maintain the lead balance with changing room temperature. The thermometer leads are connected to the bridge through selector switches that employ either mercury-wetted contacts or multiple all-silver contacts in parallel.

The cryostat is constructed so that the central core, including the copper comparison block, is stationary while the remainder of the cryostat assembly may be lowered to expose the block for thermometer installation or removal. The only seals required for sealing the cryostat are at room temperature and consist of simple Viton " $O$ " rings (E).

N. Copper plug with longitudinal grooves to serve as thermal tie-downs for thermocouples.

o. Vent.

P. Thermocouple leads to shield and thermometer block well ring heater. 


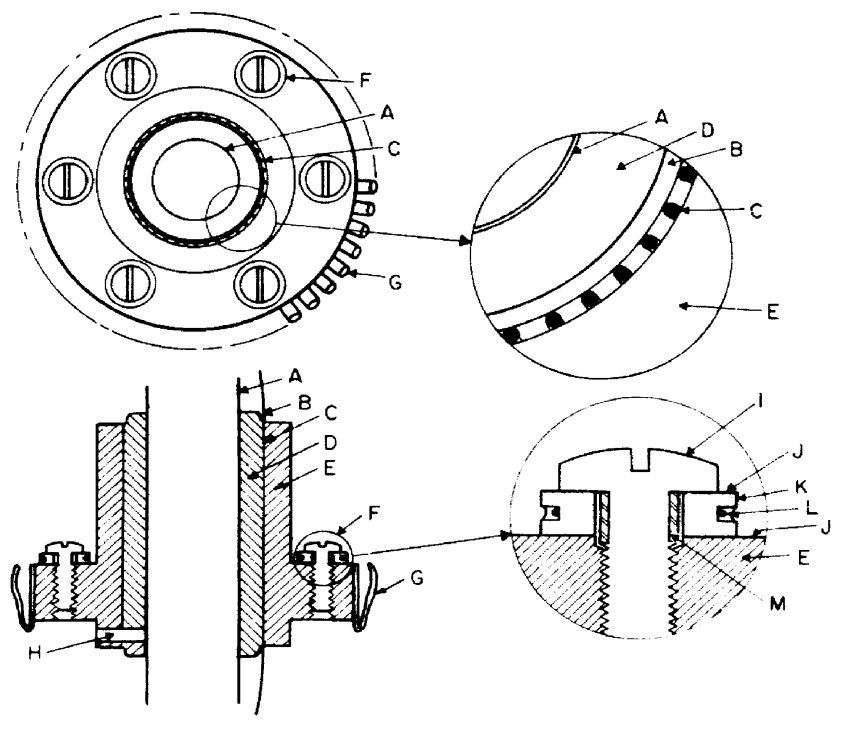

Figure 41. Thermal tie-downs.

A. Central support tube (stainless steel) for thermometer block.

B. Rounded comer, tangent to the bottom of the grooves for wire.

C. Round bottom grooves in cylinder " $D$ " with wire cemented in place.

D. Copper cylinder soldered (tin-lead eutectic) to the central support tube.

E. Copper cylinder fitted to "D" and held in place by pin "H."

F. Thermocouple tie-down.

G. Spring fingers of beryllium copper heavily silver plated and gold "flashed."

H. $1.5 \mathrm{~mm}$ steel pin to hold cylinder " $\mathrm{E}$ " in position.

I. Pan head 2-56 brass screw to clamp thermocouple tie-down assembly to "E."

J. Insulating washer of $0.005 \mathrm{~mm}$ Mylar coated with vacuum grease.

$K$ Copper washer with pre-tinned groove for thermocouple wire.

L. Polyimide insulated thernocouple wire, placed at the bottom of the pretinned groove and "potted" in eutectic tin-lead solder.

M. Epoxy insulation on screw.

The cryostat proper (fig. 38) is surrounded with the usual arrangement - an inner Dewar flask (M), which contains liquid helium, guarded by an outer Dewar flask (not shown) that contains liquid nitrogen. During the initial cool down, the inner Dewar is filled with liquid nitrogen and the liquid nitrogen vapor pressure is reduced to about $1 / 3 \mathrm{~atm}$. The vacuum can $(O)$ of the cryostat is filled with ${ }^{3} \mathrm{He}$ gas to a pressure of about 0.1 torr to facilitate the cooling: under such conditions, the thermometer block cools to about $65 \mathrm{~K}$ in approximately 12 hours (overnight). The liquid nitrogen remaining in the inner Dewar is removed by applying a small over-pressure of ${ }^{4} \mathrm{He}$ gas which forces the nitrogen out through a $3.2 \mathrm{~mm}$ thin-wall stainless tube (not shown in fig. 38) that extends from the Dewar bottom to the room. (Precooling the cryostat from about $80 \mathrm{~K}$ to about $65 \mathrm{~K}$ by pumping on liquid nitrogen reduces the liquid helium required for cooling by about 2 liters.) Six to seven liters of liquid helium are usually required to cool the apparatus from 65 to $4.2 \mathrm{~K}$ and fill the inner helium Dewar.

As a part of the calibration procedure the thermometer resistance readings are first made at about $4 \mathrm{~K}$, without the use of temperature controls. For the calibrations above $4 \mathrm{~K}$, the ${ }^{3} \mathrm{He}$ gas in the vacuum can is removed to reduce the heat transfer and the temperature of the comparison block is controlled by the use of three sets of five-junction
Chromel-P/Constantan differential thermopiles that sense temperature differences between the comparison block and the thermal shields. Appropriate automatic controls are employed to power three separate heaters to reduce the indicated temperature differences to zero. When the space in the vacuum can is evacuated to a pressure in the 10-6 torr range, most of the heat transfer to the comparison block from the surroundings is (i) down through the wall of the well $(F)$ and (ii) within the well through the central thin-wall stainless tube and electrical leads. Consequently, two sets of thermopiles and heaters are affixed, one at (R) on the wall of the well and the other at (Q) within the well. These two locations are about 15 $\mathrm{cm}$ above the comparison block. The third thermopile and heater are mounted on the heavy copper shield which surrounds the lower end of the well where the comparison block rests. The thermopiles are all referenced to the temperature of the comparison block. If adequate temperature control of a system is to be achieved, the thermal lags of both the heaters and the temperature sensors (in this case thermopiles) must be small. In addition, the "immersion error" that is caused by heat conducted along the leads of the sensor should be small. These characteristics were achieved in the cryostat by employing the "thermal tie downs" shown in figures 38 and 41 . (The selection of Chromel-P/Constantan thermocouples was influenced by their low thermal conductivity. There are other materials that possess better thermoelectric characteristics but their relatively high thermal conductivities were considered to be disadvantageous.) The spurious emf of each thermopile is balanced out with a simple emf injector that is adjustable between $\pm 5 \mu \mathrm{V}$. The adjustment for the spurious emf is made at $4.2 \mathrm{~K}$ after equilibrium has been attained with ${ }^{3} \mathrm{He}$ gas still in the can.

The calibration resistance measurements are made at 16 temperatures; approximately 12.20 . $13.81,15.426,17.042,18.661,20.28,23.691,27.102$, $33.917,40.732,47.547,54.361,66.303,78.245$, 84.216 , and $90.188 \mathrm{~K}$. The values of $W(T)$ at the IPTS-68 defining fixed-point temperatures are obtained by least square analysis of the comparison data (see sec. 6).

To change from one calibration temperature to the next higher temperature, current is supplied to the heater that is wound directly on the comparison block. Although the control of the shields is adequate to track the temperature of the block when it is rapidly heated at the lower temperatures (below about $40 \mathrm{~K}$ ), the shields lag at higher temperatures when the block is being rapidly heated because of the limitations of the power amplifiers which drive their heaters. The time required for the system to attain thermal equilibrium after the heating of the block is stopped increases with temperature. At $13.8 \mathrm{~K}$ the thermometer drift will decrease to less than $10 \mu \Omega / \mathrm{min}$ in a minute, 
while at $90 \mathrm{~K}$ more than $30 \mathrm{~min}$ is required to achieve this drift rate. Measurements are considered unsatisfactory if the indicated drift rate is more than $5 \mu \Omega /$ min at temperatures below $20 \mathrm{~K}$ or more than $10 \mu \Omega / \mathrm{min}$ at temperatures above $20 \mathrm{~K}$.

In order to achieve these low drift rates rather quickly, particularly at temperatures above $30 \mathrm{~K}$, it was found necessary to uniformly heat the lower $20 \mathrm{~cm}$ section of the well (F) when the comparison block.was being heated to the next higher calibration temperature. This was accomplished by powering a heater wound around the well $(E)$ as shown in figure 39. An adjustable fraction (depending upon the temperature) of the voltage applied to the block heater is applied to the well heater to raise its temperature at the same time and rate as the block temperature.

In the calibration of the capsule type SPRT's the resistance measurements are carried out with a continuous current of $1 \mathrm{~mA}$ through the thermometer and with a commutator switching order of NRRNNRRN with the Mueller bridge. This order of measurements is not the optimum one (which is NRRNRNNR), but when a large number of measurements must be made, such as in the calibration of thermometers over this temperature range, the pattern used is less subject to gross errors. At approximately $90.2 \mathrm{~K}$, measurements are made at two currents, $1 \mathrm{~mA}$ and $2 \mathrm{~mA}$, to determine the self heating effect of the SPRT. Although infrequent, the glass to metal seal of a thermometer may leak; this causes a loss of helium and consequent increase in the self-heating effect of the thermometer. A thermometer having this defect may be unreliable. The treatment of the data resulting from these measurements differs markedly from those obtained above $90.188 \mathrm{~K}$ (see sec. 6).

The authors gratefully acknowledge the critical review and many suggestions given by Martin $L$. Reilly which have helped to improve this monograph. They also acknowledge the help of William J. Hall who wrote the computer program for re. ducing the SPRT calibration data between 13.81 and $90.188 \mathrm{~K}$ and William R. Bigge who made the measurements for some of the illustrations.

\section{References}

[1] Alieva, F. Z., New platinum resistance thermometer for precision measurements of high temperatures, Meas. Tech. (USSR), No. 6, 487-488 (June 1964), translation from Izmeritel, Tekhn. No. 6, 21-22 (June 1964).

[2] Barber, C. R., Platinum resistance thermometers of small dimensions, J. Sci. Instr. 27, 47-49 (1950).

[3] Barber, C. R., A platinum resistance thermometer for use at low temperatures, J. Sci. Instr. 32, 416-417 (1955).

[4] Barber, C. R.. Resistance thermometers for low temperatures, in Progress in Cryogenics, Ed. K. Mendelssohn, pp. 147-171 (Academic Press, Inc., New York, N.Y., $1960)$.
[5] Barber, C. R., and Blanke, W. W.. A platinum resistance thermometer for use at high temperatures, J. Sci. Instr. 38, 17-19 (1961).

[6] Barber, C. R., Gridley, A., and Hall, J. A., A design for standard resistance coils, J. Sci. Instr. 29, 65-69 (1952).

[7] Barber, C. R., Gridley, A., and Hall, J. A., An improved construction of the Smith bridge, type 3, J. Sci. Instr. 32, 213-220 (1955).

[8] Bedford, R. E., Durieux, M., Muijlwijk, R., and Barber, C. R., Relationships between the International Practical Temperature Scale of 1968 and the NBS-1955, NPL-61, PRMI-54, and PSU-54 Temperature scales in the range from 13.81 to $90.188 \mathrm{~K}$, Metrologia 5, No. 2, 47-49 (April 1969).

[9] Bedford, R. E., and Ma, C. K., A note on the reproducibility of the IPTS- 68 below $273.15 \mathrm{~K}$, Metrologia 6, No. 3, 89-94 (1970).

[10] Behr, L., The Wenner potentiometer, Rev. Sci. Instr. 3, No. 3, 109-120 (1932).

[11] Belyansky, L. B., Orlova, M. P., Sharevskaya, D. I., and Astrov, D. N., Investigation of the resistance-temperature properties of platinum for resistance thermometry over the range from $90 \mathrm{~K}$ to $273 \mathrm{~K}$. Metrologia 5, No. 4, 107-111 (1969).

[12] Berry, R. J., The temperature-time dependence of the triplepoint of water, Can. J. Phys. 37, 1230-1248 (1959).

[13] Brickwedde, F. G., van Dijk, H., Durieux, M., Clement. J. R., and Logan, J. K., The " $1958{ }^{4} \mathrm{He}$ Scale of Temperature," J. Res. Nat. Bur. Stand. (U.S.) 64A (Phys. and Chem.), No. 1, 1-17 (1960).

[14] Brooks, P. P. B., Calibration procedures for direct-current resistance apparatus, Nat. Bur. Stand. (U.S.), Monogr. 39,53 pages (March 1,1962 ).

[15] Burgess, G. K., The International Temperature Scale, J. Res. Nat. Bur. Stand. (U.S.) 1, 635-640 (1928)RP22.

[16] Callendar, H. L., On the practical measurement of temperature: Experiments made at the Cavendish Laboratory, Cambridge, Phil. Trans. (London) 178, 161-230 (1887).

[17] Comité International des Poid et Mesures, Procès-verbaux des séances de l'année 1954, 24, 81-82, T20-T23, T44-T47 (1955).

[18] Curtis, D. J., and Thomas, G. J.. Long term stability and performance of platinum resistance thermometers for use to $1063{ }^{\circ} \mathrm{C}$, Metrologia 4, No. 4, 184-190 (October 1968).

[19] Cutkosky, R. D., An a-c resistance thermometer bridge. J. Res. Nat. Bur. Stand. (U.S.) 74C (Engr. and Instr.) Nos. 1 and 2, 15-18 (Jan.-June 1970).

[20] Dauphinee, T. M., Potentiometric methods of resistance measurement, in American Institute of Physics, Tempera ture, Its Measurement and Control in Science and Indus try, Vol. 3, Part 1, 269-283 (Reinhold Publishing Corp. New York, N.Y., 1962).

[21] Eppley, M., Modifications of the resistance thermometer bridge (Mueller bridge) and of the commutator selector for use with it, Rev. Sci. Instr. 3, 687-711 (1932).

[22] Evans, J. P., An improved resistance thermometer bridge, in American Institute of Physics, Temperature, Its Mea surement and Control in Science and Industry, Vol. 3 , Part 1, 285-289 (Reinhold Publishing Corp., New York. N.Y., 1962).

[23] Evans, J. P., and Burns, G. W., A Study of Stability of High Temperature Platinum Resistance Thermometers, in American Institute of Physics. Temperature, Its Measure ment and Control in Science and Industry, Vol. 3, Part 1, 313-318 (Reinhold Publishing Corp., New York. N.Y., 1962).

[24] Evans, J. P., and Wood, S. D., An intercomparison of high temperature platinum resistance thermometers and standard thermocouples, Metrologia 7, No. 3, 108-130 (July 1971).

[25] Evans, J. P., and Sweger, D. M., Immersion cooler for freezing ice mantles on triple-point-of-water cells, Rev. Sci. Instr. 40, No. 2, 376-377 (1969).

[26] Furukawa, G. T., Automation problems in thermometry and calorimetry, in Netsu, Ondo Sokutei, to Shisanetsu 
Bunseki, 1969 (Calorimetry, Thermometry, and Differential Thermal Analysis, 1969), Eds: Y. Otsubo, H. Kanbe, and S. Seki (Kagaku Gijitsu Sha, Tokyo, 1969).

[27] Gautier, M., A modification of the Smith Bridge, type III. J. Sci. Instr. 30, 381-382 (1953).

[28] Hoge, H. J., and Brickwedde, F. G., Establishment of a temperature scale for the calibration of thermometers between 14 and $83^{\circ} \mathrm{K}$, J. Res. Nat. Bur. Stand. (U.S.) 22, 351-373 (1939) RP1158.

[29] Hoge, H. J., and Brickwedde, F. G., Intercomparison of platinum resistance thermometers between -190 and $445^{\circ} \mathrm{C}$, J. Res. Nat. Bur. Stand. 28, 217-240 (Feb. 1942) RP1454.

[30] Iwase, K., Aoki, N., and Osawa, A., The equilibrium diagram of the tin-antimony system and the crystal structure of the $\boldsymbol{\beta}$-solid solution, Sci. Reports Tohoku Imp. Univ. (Series 1) 20, 351-368 (1931).

[31] Johnson, J. B., Thermal agitation of electricity in conductors, Phys. Rev. 32, 97-109 (July 1928).

[32] McLaren, E. H., The freezing points of high purity metals as precision temperature standards. II. An investigation of the freezing temperatures of zinc, cadmium, and tin, Can. J. Phys. 35, 1086-1106 (1957).

33] McLaren, E. H., Intercomparison of 11 resistance ther mometers at the ice, steam, tin, cadmium, and zinc points, Can. J. Phys. 37, 422-432 (1959).

[34] Meyers, C. H., Coiled filament resistance thermometers, J. Res. Nat. Bur. Stand. (U.S.) 9, 807-817 (December 1932)RP508.

[35] Michaelis, R. E., Office of Standard Reference Materials (NBS), private communication.

[36] Moussa, M. R. M., Van Dijk, H., and Durieux, M., Comparison of platinum resistance thermometers between $63 \mathrm{~K}$ and $373.15 \mathrm{~K}$, Physica 40, 33-48 (1968).

[37] Mueller, E. F., Wheatstone bridges and some accessory apparatus for resistance thermometry, Sci. Papers Bur. Stands. 13, 547-561 plus 1 plate $(1916-1917)$.

[38] Mueller, E. F., Precision resistance thermometry, in American Institute of Physics, Temperature, Its Measurement and Control in Science and Industry, Vol. 1, pp. 162-179 (Reinhold Publishing Corp., New York, N.Y., 1941).

[39] Mueller, E. F., and Wenner, F., The Waidner-Wolff and other adjustable electrical-resistance elements, J. Research Natl. Bur. Stand. 15, 477-492 (Nov. 1935)RP842.

[40] Pfann, W. G., Zone Melting, second edition, (Wiley and Sons, New York, N.Y., 1966).

[41] Rosebury, F., Handbook of Electron Tube and Vacuum Techniques, p. 371 (Addison-Wesley Publishing Co., Inc., Reading, Massachusetts, 1965).

[42] Saylor, C. P., Calculation of cryoscopic data, Anal. Chim. Acta 17, 36-42 (1957).
[43] Schaeffer, W. H., The six-dial thermofree potentiometer, Instruments and Control Systems 34, No. 2, 283-291 (Feb. 1961).

[44] Sharevskaya, D. I., Orlova, M. P., Belyansky, L. B., and Galoushkina, G. A., Investigation of the resistancetemperature properties of platinum of resistance thermometry over the range from $14 \mathrm{~K}$ to $90 \mathrm{~K}$, Metrologia 5 , No. 4, 103-107 (1969).

[45] Smit, W. M., Errors occurring in the determination of temperature-heat content curves, Anal. Chim. Acta 17, 23-42 (1957)

[46] Stimson, H. F., The International Temperature Scale of 1948, J. Res. Nat. Bur. Stand. (U.S.) 42, 209-217 (1948) RP1962.

[47] Stimson, H. F., Precision resistance thermometry and fixed points, in American Institute of Physics, Temperature, Its Measurement and Control in Science and Industry, Vol. 2, 141-168 (Reinhold Publishing Corp., New York, N.Y., 1955).

[48] Stimson, H. F., International Practical Temperature Scale of 1948. Text revision of 1960 , J. Res. Nat. Bur. Stand. (U.S.), 65A (Phys. and Chem.), No. 3, 139-145 (MayJune 1961).

[49] Strelkov, P. G., Borovik-Romanov, A. S., and Orlova, M. P., Thermodynamic studies at low temperatures. I. Measurement of temperatures between 12 and $300{ }^{\circ} \mathrm{K}, \mathrm{Zh}$. Fiz. Khim. 28, 345-352 (1954).

[50] Swindells, J. F., National Bureau of Standards Provisional Scale of 1955, in Precision Measurement and Calibration. Temperature, Nat. Bur. Stand. (U.S.), Spec. Publ. 300, Vol. 2, 513 pages (Aug. 1968), p. 56.

[51] The International Practical Temperature Scale of 1968, Adopted by the Comité International des Poids et Mesures, Metrologia 5, No. 2, 35-44 (Apr. 1969).

[52] Toenshoff, D. A., An evaluation of some factors which may affect the stability of birdcage thermometers, report of work conducted under Contract No. CST383 (National Bureau of Standards) by Engelhard Industries (East Newark, N.J. 07029), 44 pages, January 1, 1968.

[53] Unpublished test measurements at NBS with manually controlled copper block furnaces suitable for conducting metal freezing and melting measurements.

[54] White, W. P., Potentiometers for thermoelectric measurements especially in calorimetry, J. Am. Chem. Soc. 36, 1868-1885 (1914).

[55] White, W. P., Einige neu Doppelkompensatoren. I. Thermokraftfreie Kontakte, mechanische Anordnungen und Zubehörteile, Zeit. Instrumentenk. 34, 71-82 (1914).

[56] White, W. P., Einige neue Doppelkompensatoren. II. Die elektrische Einrichtung. Zeit. Instrumentenk. 34, 107$113,142-151$ (1914). 


\title{
9. Appendixes
}

\section{APPENDIX A.}

\section{The International Practical Temperature Scale of 1968*}

\author{
Adopted by the Comité International des Poids et Mesures
}

Rereived February 17, 1969

\section{Foreword}

The Comite International des Poids et Mesures (CIPM) at its meeting in October 1968 agreed to adopt the International Practical Temperature sicule of 1968 (IPTS-68) in accordance with the decision of the $133^{r}$ Conférence Générale des Poids et Mesures, Resolution 8 . of October 1967. This resolution gave the CIPM permission to introduce a new Scale to replace the existing IPTS-48 as soon as the Comité Consultatif de Thermométrie (CCT) made its recommendations.

There were two important reasons for revising the IPTS-4ه. The first of these was the need to extend the scale to lower temperature; to achieve a unification of the existing national scales in the region $10 \mathrm{~K}-90 \mathrm{~K}$. Siecondly the Scale had not been revised significantly over the range from $-183^{\circ} \mathrm{C}$ to $1063 \mathrm{C}$ since its inception in 1927. and modern gas thermometer measurements showed that the Sicale gave values considerably different from thermodvnamic temperatures.

The CC'T agreed on the basic data and methods of interpolation for a new definition of the IP'T's at its 8th meeting held in Washington and (Httawa in September 1967. Provision was made to extend the Scale down to $13.81 \mathrm{~K}$. the triple point of equilibrium hydrogen. and for the use of the best known values of thermodynamic temperatures. The value of c., used in the Planck equation was also revised as a result of a better knowledge of the values of fundiamental physical constants. The (') T appointed a small sub-committee to prepare a text of the definition of the IPT'S.68 in aceordance with its recom. mendations. with instructions to resolve some points of detail. The final draft of the English text, which is reproduced below. received the approval of all members of the (CT before it was submitted to the CIPM. The official text of the IPTS-68 is the French text which was prepared by the Bureaul International des Poids et Mesures from the English text. and is published as an annexe to Comptes rendus des séances de la Treizième Conférence Giénérale des Poids et Mesures.

C. R. ВIRKER

\section{Introduction}

The basic temperature is the thermodynamic: temperature, symbol $T$, the unit of which is the kelvin, symbol $k$. The kelvin is the fraction $1 / 273.16$ of the thermodynamic temperature of the triple point of watei'

The Celsius temperature. symbol $t$, is defined by

$$
t=T-T_{\mathrm{o}}
$$

where $T_{0}=273.15 \mathrm{~K}$. The unit employed to express a Celsius temperature is the degree ('elsius, symbol ' $('$ '. which is equal to the kelvin. A difference of temperature is expressed in kelvins; it may also be expressed in degrees Celsius.

* The text in French of this scale is published in Comptes rendus de la Treizième Conférence Générale des Poids et Mesures, 1967-1968, Annexe 2. and Comité Consultatif de Thermométrie, 8 r session, 1967, Annexe 18 .

13 th General Conference of Weights and Measures (196i), Resolutions 3 and 4.
The International Practical Temperature soale of 1968 (IPTS-68) has been chosen in such a way that the temperature measured on it closely approximates the thermodynamic temperature: the difference is within the limits of the present accuracy of measurement.

The International Practical Temperature Scale of $196 \%$ distinguishes between the International Practical Kelvin Temperature with the symbol $T_{G B}$ and the International Practical Celsius Temperature with the symbol $t_{68}$; the relation between $T_{68}$ and $t_{6 x}$ is

$$
t_{68}=: T_{68}-273.15 \mathrm{~K} .
$$

The units of $T_{68}$ and $t_{68}$ are the kelvin. symbol $K$. and degree Celsius, symbol ${ }^{\circ} \mathrm{C}$, as in the case of the thermodynamic temperature $T$ and the Celsius temperature $t$.

The International Practical Temperature Scale of 1968 was adopted by the International ('ommittee of Weights and Measures at its meeting in 196s according to the power given to it by Resolution $s$ of the 13th General Conference of Weights and Heasures. It replaces the International Practical 'Temperature scale of 194s (amended erlition of 1960 ).

\section{Definition of the International} Practical 'Temperature Scalle of $196 \mathrm{~K}^{2}$ (IP'Ts-6s)

\section{Principle of the IPTS-6is amd Defining Fired Points}

'The IPTS-6s is based on the assigned values of the temperatures of a number of reproducible equilibrium states (defining fixed points) and on standard instruments calibrated at those temperatures. Interpolation between the fixed point temperatures is provided by formulae used to establish the relation between indieations of the standard instruments and values of International Practical Temperature.

The defining fixed points are establishod by realizing specified equilibrium states between phases of pure substances. These equilibrium states and the values of the International Practical Temperature assigned to them are given in 'Table 1.

The standard instrument used from $|3 . s|$ K to $630.74^{\circ}($ is the platinum resistanee thermometer. 'Whe thermometer resistor must be strain-free, annealed pure platinum. 'Ihe resistance atio $\mathrm{H}^{\prime}\left(T_{6 x}\right)$, defined by

$$
W\left(T_{68}\right)=: R\left(T_{6 \mathrm{~B}}\right) /(P(273.15 \mathrm{~K})
$$

"In this document Kelvin temperatures are: used. in grencer. al, below 0 "C and Celsius temperatures are used above 0 (') This avoids the use of negative values and confornm with general usage. 
Table 1. Defining fixed points of the IPTS-f;8*

\begin{tabular}{|c|c|c|}
\hline \multirow[t]{2}{*}{ Equilibrium state } & \multicolumn{2}{|c|}{$\begin{array}{l}\text { Assigned value of } \\
\text { International } \\
\text { Practical } \\
\text { Temperature }\end{array}$} \\
\hline & $\overline{T_{B 8}}(\bar{K})$ & $t_{68}\left({ }^{\circ} \mathrm{C}\right)$ \\
\hline $\begin{array}{l}\text { Equilibrium between the solid, liquid } \\
\text { and vapour phases of equilibrium } \\
\text { hydrogen (triple point of equilibrium } \\
\text { hydrogen) }\end{array}$ & 13.81 & -259.34 \\
\hline $\begin{array}{l}\text { Equilibrium between the liquid and } \\
\text { vapour phases of equilibrium } \\
\text { hydrogen at a pressure of } \\
33330.6 \mathrm{~N} / \mathrm{m}^{2} \text { (25/76 standard } \\
\text { atmosphere) }\end{array}$ & .17 .042 & -256.108 \\
\hline $\begin{array}{l}\text { Equilibrium between the liquid and } \\
\text { vapour phases of equilibrium } \\
\text { hydrogen (boiling point of } \\
\text { equilibrium hydrogen) }\end{array}$ & 20.28 & -252.87 \\
\hline $\begin{array}{l}\text { Equilibrium between the liquid and } \\
\text { vapour phases of noon (boiling point } \\
\text { of neon) }\end{array}$ & 27.102 & $-246.04 x$ \\
\hline $\begin{array}{l}\text { Equilibrium between the solid, liquid } \\
\text { and vapour phases of oxygen (triple } \\
\text { point of oxygen) }\end{array}$ & 54.361 & -218.789 \\
\hline $\begin{array}{l}\text { Equilibrium between the liquid and } \\
\text { vapour phases of oxygen (boiling } \\
\text { point of oxygen) }\end{array}$ & 90.188 & -182.962 \\
\hline $\begin{array}{l}\text { Equilibrium between the solid, liquid } \\
\text { and vapour phases of water (triple } \\
\text { point of water) }\end{array}$ & 273.16 & 0.01 \\
\hline $\begin{array}{l}\text { Equilibrium between the liquid and } \\
\text { vapour phases of water (boiling } \\
\text { point of water) }\end{array}$ & 373.15 & 100 \\
\hline $\begin{array}{l}\text { Equilibrium between the solid and } \\
\text { liquid phases of zinc (freezing point } \\
\text { of zinc) }\end{array}$ & 692.73 & 419.58 \\
\hline $\begin{array}{l}\text { Equilibrium between the solid and } \\
\text { liquid phases of silver (freezing point } \\
\text { of silver) }\end{array}$ & 1235.08 & 961.93 \\
\hline $\begin{array}{l}\text { Equilibrium between the solid and } \\
\text { liquid phases of gold (freezing point } \\
\text { of gold) }\end{array}$ & 1337.58 & 1064.43 \\
\hline
\end{tabular}

- Except for the triple points and one equilibrium hydrogen point $(17.042 \mathrm{~K}$ ! the assigned values of temperature are for equilibrium states at a pressure $p_{o}=1$ standard atmosphere (101 $325 \mathrm{~N} / \mathrm{m}^{2}$ ). In the realization of the fixed points small departures from the assigned temperatures will occur as a result of the differing immersion depths of thermometers or the failure to realize the required pressure exactly. If due allowance is made for these small temperature differences. they will not affect the accuracy of realization of the Scale. The magnitudes of these differences are given in section III.

b The equilibrium state between the solid and liquid phases of tin (freezing point of tin) has the assigned value of $t_{68}=231.9681^{\circ} \mathrm{C}$ and may be used as an alternative to the boiling point of water.

c The water used should have the isotopic composition of ocean water, see section III. 4.

where $R$ is the resistance, must not be less than 1.39250 at $T_{68}=373.15 \mathrm{~K}$. Below $0^{\circ} \mathrm{C}$ the resistancetemperature relation of the thermometer is found from a reference function and specified deviation equations. From $0^{\circ} \mathrm{C}$ to $630.74^{\circ} \mathrm{C}$ two polynomial equations provide the resistance-temperature relation.

The standard instrument used from $630.74{ }^{\circ} \mathrm{C}$ to $1064.43{ }^{\circ} \mathrm{C} !$ is the platinum-10\% rhodium/platinum thermocouple, the electromotive force-temperature relation of which is represented by a quadratic equa. tion.
Above $1337.58 \mathrm{~K}\left(1064.43{ }^{\circ}(\mathrm{c})\right.$ the International Practical Temperature of 1968 is defined by the Planck law of radiation with $1337.58 \mathrm{~K}$ as the reference temperature and a value of $0.0143 \mathrm{ks}$ metre kelvin for $c_{2}$.

\section{Definition of the International I'ractical Temperature of $196 \mathrm{~S}$ in Different Temperature Runges}

a) The range from $13.81 \mathrm{~K}$ to $273.15 \mathrm{~K}$

From $13.81 \mathrm{~K}$ to $273.15 \mathrm{~K}$ the temperature $T_{68}$ is defined by the relation

$$
W\left(T_{68}\right)=W_{\text {CC. T-68 }}\left(T_{68}\right)+\Delta W\left(T_{68}\right)
$$

where $W\left(T_{68}\right)$ is the resistance ratio of the platinum resistance thermometer and $W_{\mathrm{CCT} \cdot \mathrm{GN}}\left(T_{\mathrm{BR}}\right)$ is the resistance ratio as given by the reference function set out in Table $2^{3}$. The deviations $\Delta W\left(T_{68}\right)$ at the temporatures of the defining fixed points are obtained from the mcasured values of $W\left(T_{68}\right)$ and the corresponding values of $W_{\text {CC'T-68 }}\left(T_{68}\right)$, see 'Table 4 . 'To find $J W\left(T_{68}\right)$ at intermediate temperatures interplation formulac are used. The range betweon $13.5 \mid \mathrm{K}$ and $273.15 \mathrm{~K}$ is divided into four parts in calch of which $\Delta W\left(T_{G N}\right)$ is defined by a polynomial in $T_{68}$. The constants in the polynomials are determined from the values of $\Delta W\left(T_{68}\right)$ at the fixed points and the condition that there should be no discontinuity in $\mathrm{d} \Delta W\left(T_{68}\right) / \mathrm{d} T_{G 8}$ at the junctions of the temperature ranges.

From $13.81 \mathrm{~K}$ to $20.2 \mathrm{~K}$ the deviation function is

$$
\Delta W\left(T_{68}\right)=A_{1}+B_{1} T_{68}+C_{1} T_{68}^{2}+J_{1} T_{6 \times}^{3}
$$

where the constants are determined by the measured deviations at the triple point of ecquilibrium hydrogen, the temperature of $17.042 \mathrm{~K}$ and the boiling point of equilibrium hydrogen and by the derivative of the deviation function at the boiling point of equilibrium hydrogen as derived from Eq. (6).

From $20.28 \mathrm{k}$ to $54.361 \mathrm{~K}$ the deviation function is

$$
\Delta W\left(T_{68}\right)=A_{2}+B_{2} T_{68}+C_{2} T_{68}^{2}+J_{2} T_{68}^{3}
$$

where the constants are determined by the moasured deviations at the boiling point of equilibrium hyilogen, the boiling point of neon and the triple point of oxygen and by the derjuative of the deviation function at the triple point of oxygen as derived from Eq. (7).

From $54.361 \mathrm{~K}$ to $90.1 \times \mathrm{s} \mathrm{K}$ the deviation function is

$$
\Delta W\left(T_{6 \mathrm{H}}\right): \cdots A_{3}+B_{3} T_{64}+C_{3} T_{68}^{2}
$$

where the constants are determined by the measured deviations at the triple point and the boiling point of oxygen and by the derivative of the deviation function at the boiling point of oxygen as derived from Eq. (8).

From $90.188 \mathrm{~K}$ to $273.15 \mathrm{~K}$ the deviation function is

$$
\Delta W\left(T_{68}\right) \cdots A_{4} t_{68}+C_{4} t_{68}^{3}\left(t_{68} \cdots 100^{\circ}\left(^{\circ}\right)\right.
$$

where $t_{6 \mathrm{~B}}=T_{6 \mathrm{~F}}-273.15 \mathrm{~K}$ and the constants are determined by the measured deviations at the boiling point of oxyeren and the boiling point of water"

i For the relation between the IPTsi-6s and the "national scales" from which it was in part derived see Appendix I.

4 If the freezing point of tin (see Sote ". Table 1) is used as a fixed point instead of the boiling point of water, $W^{\circ}(100)^{\circ}()$ for the platinum thermometer should be calculated from Fqs. (9) and (10). 
Table 2. The reference function $W_{\text {cct-as }}\left(T_{\Delta 8}\right)$ for platinum resistance thermometers for the range from $1.3 .81 \mathrm{~K}$ to $273.15 \mathrm{~K}$.

Coefficients $A_{i}$

$$
T_{68}=\left\{A_{0}+\sum_{i=1}^{20} A_{t}\left[\ln W_{\mathrm{CCT}-68}\left(T_{68}\right)\right]^{4}\right\} \mathrm{K}
$$

\begin{tabular}{|c|c|c|c|}
\hline$i$ & $A_{1}$ & $i$ & $A_{1}$ \\
\hline 0 & $0.27315 \times 10^{3}$ & 11 & $0.7679763581708458 \times 10$ \\
\hline 1 & $0.2508462096788033 \times 10^{3}$ & 12 & $0.2136894593828500 \times 10$ \\
\hline 2 & $0.1350998699649997 \times 10^{3}$ & 13 & 0.4598433489280693 \\
\hline 3 & $0.527 \times 567590085172 \times 10^{2}$ & 14 & $0.7636146292316480 \times 10^{-1}$ \\
\hline 4 & $0.2767685488541052 \times 10^{2}$ & 15 & $0.9693286203731213 \times 10^{-2}$ \\
\hline 5 & $0.3910532053766837 \times 10^{2}$ & 16 & $0.9230691540070075 \times 10^{-3}$ \\
\hline 6 & $0.6556132305780693 \times 10^{2}$ & 17 & $0.6381165909526538 \times 10^{-4}$ \\
\hline 7 & $0.8080358685598667 \times 10^{2}$ & 18 & $0.302293237 \times 746192 \times 10^{-5}$ \\
\hline 8 & $0.7052421182340520 \times 10^{2}$ & 19 & $0.8775513913037602 \times 10^{-7}$ \\
\hline 9 & $0.4478475896389657 \times 10^{2}$ & 20 & $0.1177026131254774 \times 10^{-8}$ \\
\hline 10 & $0.2125256535560578 \times 10^{2}$ & & \\
\hline
\end{tabular}

The reference function $W_{\mathrm{CCT}-\mathrm{Bs}}\left(T_{\mathrm{Bg}}\right)$ is continuous at $T_{88}=273.15 \mathrm{~K}$ in its first and second derivatives with the function $W\left(t_{68}\right)$ given by Eqs. $(9)$ and $(10)$ for $\alpha=$ $3.9259668 \times 10^{-3}{ }^{\circ} \mathrm{C}^{-1}$ and $\delta=1.496334{ }^{\circ} \mathrm{C}$.

- A tabulation of this reference function, sufficiently detailed to allow interpolation to an accuracy of $0.0001 \mathrm{~K}$, is available from the Bureau International des Poids et Mesures, 92-Sèvres, France. A skeleton tabulation appears in this text as Table 3.

b) The range from $0^{\circ} \mathrm{C}(273.15 \mathrm{~K})$ to $630.74^{\circ} \mathrm{C}$ From 0 or, to $630.74^{\circ} \mathrm{C} t_{68}$ is defined by

$$
\begin{gathered}
t_{6 \mathrm{~B}}=t^{\prime}+0.045\left(\frac{t^{\prime}}{100^{\circ} \mathrm{C}}\right)\left(\frac{t^{\prime}}{100{ }^{\circ} \mathrm{C}}-1\right) \\
\cdot\left(\frac{t^{\prime}}{419.588^{\circ} \mathrm{C}}-1\right)\left(\frac{t^{\prime}}{630.74^{\circ} \mathrm{C}}-1\right){ }^{\circ} \mathrm{C}
\end{gathered}
$$

where $t^{\prime}$ is defined by the equation:

$$
t^{\prime}=\frac{1}{\alpha}\left[W\left(t^{\prime}\right)-1\right]+\delta\left(\frac{t^{\prime}}{100^{\circ} \mathrm{C}}\right)\left(\frac{t^{\prime}}{100^{\circ} \mathrm{C}}-1\right)
$$

where $W\left(t^{\prime}\right)=\frac{R\left(t^{\prime}\right)}{R\left(0^{\circ} \mathrm{C}\right)}$. The constants $R\left(0^{\circ} \mathrm{C}\right)$, $\alpha$ and $\delta$ are determined by measurement of the resistance at the triple point of water, the boiling point of water (or the freezing point of tin, see Note ${ }^{b}$, Table 1) and the freezing point of zinc.

Eq. $(10 a)$ is equivalent to the equation

$$
W\left(t^{\prime}\right)=1+A t^{\prime}+B t^{\prime 2}
$$

where $A=\alpha\left(1+\delta / 100^{\circ} \mathrm{C}\right)$ and $B=-10^{-4} \alpha \delta^{\circ} \mathrm{C}^{-2}$.

c) The range from $630.74{ }^{\circ} \mathrm{C}$ to $1064.43^{\circ} \mathrm{C}$

From $630.74{ }^{\circ} \mathrm{C}$ to $1064.43{ }^{\circ} \mathrm{C} t_{68}$ is defined by the equation

$$
E\left(t_{68}\right)=a+b t_{68}+c t_{68}^{2}
$$

where $E\left(t_{68}\right)$ is the electromotive force of a standard thermocouple of rhodium-platinum alloy and platinum, when one junction is at the temperature $t_{68}=$ $0{ }^{\circ} \mathrm{C}$ and the other is at temperature $t_{68}$. The constants $a, b$ and $c$ are calculated from the values of $E$ at $630.74^{\circ} \mathrm{C} \pm 0.2^{\circ} \mathrm{C}$, as determined by a platinum resistance thermometer, and at the freezing points of silver and gold.

The wires of the standard thermocouple shall be annealed and the purity of the platinum wire shall be such that the ratio $W\left(100^{\circ} \mathrm{C}\right)$ is not less than 1.3920 . The rhodium-platinum wire shall contain nominally $10 \%$ rhodium and $90 \%$ platinum by weight. The thermocouple shall be such that the electromotive forces $E\left(630.74^{\circ} \mathrm{C}\right), E(\mathrm{Ag})$ and $E(\mathrm{Au})$ shall satisfy the following relations:

$$
\begin{gathered}
E(\mathrm{Au})=10300 \mu \mathrm{V} \pm 50 \mu \mathrm{V} \\
E(\mathrm{Au})-E(\mathrm{Ag})=1183 \mu \mathrm{V} \\
+0.158[E(\mathrm{Au})-10300 \mu \mathrm{V}] \pm 4 \mu \mathrm{V} \\
E(\mathrm{Au})-E\left(630.74^{\circ} \mathrm{C}\right)=4766 \mu \mathrm{V} \\
+0.631[E(\mathrm{Au})-10300 \mu \mathrm{V}] \pm 8 \mu \mathrm{V}
\end{gathered}
$$

d) The range above $1337.5 \mathrm{~S} \mathrm{~K}\left(1064.43^{\circ} \mathrm{C}\right)$

Above $1337.58 \mathrm{~K}\left(1064.43^{\circ} \mathrm{C}\right)$ the temperature $T_{68}$ is defined by the equation

$$
\frac{L_{\lambda}\left(T_{68}\right)}{L_{\lambda}\left(T_{68}(A u)\right)}=\frac{\exp \left[\frac{c_{2}}{\lambda T_{68}(A u)}\right]-1}{\exp \left[\frac{c_{2}}{\lambda T_{68}}\right]-1}
$$

in which $L_{\lambda}\left(T_{68}\right)$ and $L_{\lambda}\left(T_{68}(\mathrm{Au})\right)$ are the spectral concentrations at temperature $T_{68}$ and at the freezing point of gold, $T_{68}(\mathrm{Au})$, of the radiance of a blackbody at the wavelength $\lambda^{5} ; c_{2}=0.014388$ metre kelvin.

\section{Supplementary Information}

The apparatus, methods and procedures described in this section represent good practice at the present time.

\section{Standard Resistance Thermometer}

A standard platinum resistance thermometer should be so designed and constructed that the fourterminal resistance element is as free as possible from strain and will remain so in use. Satisfactory resistors have been made with platinum wires of uniform diameter between 0.05 and $0.5 \mathrm{~mm}$ and with at least a short portion of each lead adjacent to the resistor also made of platinum. A commonly used value of $R\left(0^{\circ} \mathrm{C}\right)$ is $\approx 25 \mathrm{ohms}$ and the measuring current for such a thermometer is normally 1 or 2 milliamperes. All thermometer components in close proximity to the resistor must be clean and non-reactive with platinum.

${ }^{5}$ Since $T_{\mathrm{BB}}(\mathrm{Au})$ is close to the thermodynamic temperature of the freezing point of gold and $c_{2}$ is close to the sccond radiation constant of the Planck equation, it is not necessary to specify the value of the wavelength to be employed in the measurements [see Metrologia 3, 28 (1967)]. 
Table 3. Values of $W_{\mathrm{CCT}-88}\left(T_{68}\right)$, accurding to the equation given in Table :? at inteyral values of $T_{68}$

\begin{tabular}{|c|c|c|c|c|c|c|c|c|c|c|c|}
\hline $\begin{array}{l}T_{68} \\
\mathrm{~K}\end{array}$ & $W_{\text {CCT }-68}\left(T_{68}\right)$ & $\begin{array}{l}T_{68} \\
\mathrm{~K}\end{array}$ & $W_{\mathrm{CCT}-68}\left(T_{68}\right)$ & $\begin{array}{l}f_{68}^{\prime} \\
\mathrm{K}\end{array}$ & $W_{\text {CCT } 68}\left(T_{68}\right)$ & $\begin{array}{l}T^{\prime} 88 \\
\mathbf{h}^{2}\end{array}$ & $W_{\operatorname{cct} 68}\left(T_{68}\right)$ & $T_{\mathrm{BH}}$ & $W_{\mathrm{CCT} \cdot 68}\left(T_{68}\right)$ & $T_{B R}$ & $W_{0768}\left(T_{v 8}\right)$ \\
\hline & & 50 & 0.07537756 & 100 & $0.2 \$ 630201$ & 150 & 0.49861135 & 200 & 0.70496694 & 2.30 & 0.90735309 \\
\hline & & 51 & 0.07910123 & 101 & 0.290621 .54 & 151 & $0.5027 \times 707$ & 201 & 0.70905004 & 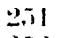 & 0.911397 .33 \\
\hline & & 52 & 0.08287595 & 102 & $0.29493 \mathrm{~S} 41$ & 152 & $0.506 ; 96058$ & 202 & 0.71313161 & 252 & $0.9154 .607 t$ \\
\hline & & 53 & $0.0 \$ 669859$ & 103 & 0.29925245 & 153 & 0.51113172 & 203 & 0.71721174 & 253 & $0.919+2.74$ \\
\hline & & 54 & 0.09056600 & 104 & 0.30356359 & 154 & 0.51530065 & 204 & 0.721290126 & 2.54 & $0.923+3+33+33$ \\
\hline & & 55 & 0.09447515 & 105 & 0.30787183 & 155 & 0.51946737 & 205 & 0.72536733 & 25.5 & $0.927+428.3$ \\
\hline & & 56 & 0.09842336 & 106 & 0.31217710 & 156 & 0.52363180 & 206 & 0.72944288 & $2 \sin 6$ & $0 . \$ 3145103$ \\
\hline & & 57 & 0.10240774 & 107 & 0.31647939 & 157 & 0.52779409 & 207 & 0.73351690 & 2.77 & ת. \\
\hline & & 58 & 0.10642583 & 108 & 0.32077856 & 158 & 0.5319 .1417 & 208 & 0.737 .58947 & 2.58 & $0.939+6.371$ \\
\hline & & 59 & 0.11047506 & 109 & 0.32507467 & 159 & 0.53611211 & 209 & 0.74166059 & 259 & $0.94346 \times 22$ \\
\hline & & 60 & 0.11455312 & 110 & 0.32936765 & 160 & 0.54026792 & 210 & 0.74573026 & $\simeq 60$ & $0.947 \pm 7152$ \\
\hline & & 61 & 0.11865789 & 111 & 0.33365751 & 161 & 0.54442167 & 211 & 0.74979841 & 261 & $0.951473 \quad 25$ \\
\hline & & 62 & 0.12278722 & 112 & 0.33794416 & 162 & 0.548 .57336 & 212 & 0.75386 .518 & $26: 2$ & $0.955+7430$ \\
\hline 13 & 0.00123061 & 63 & 0.12693914 & 113 & 0.34222768 & 163 & 0.5527291 & 213 & 0.75793043 & 263 & $0.959+738.5$ \\
\hline 14 & 0.00145973 & 64 & 0.13111189 & 114 & 0.34650800 & 164 & 0.55687046 & 214 & 0.76199430 & 264 & 0.96347219 \\
\hline 15 & 0.00174541 & 65 & 0.13530363 & 115 & 0.35078519 & 165 & 0.56101606 & 215 & 0.76605672 & $26 i$ & 0.96746931 \\
\hline 16 & 0.00209474 & 66 & 0.13951284 & 116 & 0.35505910 & 166 & 0.56515958 & 216 & 0.77011770 & 266 & 0.97146513 \\
\hline 17 & 0.00251512 & 67 & 0.14373800 & 117 & 0.359328989 & 167 & 0.56930112 & 217 & $0.77+17730$ & 267 & 0.4754 .5980 \\
\hline 18 & 0.00301428 & 68 & 0.14797773 & 118 & 0.36359754 & $16 s$ & $0.573+44076$ & 218 & 0.77523545 & $26 \mathrm{~s}$ & 0.97945325 \\
\hline 19 & 0.00359962 & 69 & 0.15223058 & 119 & 0.36786199 & 169 & אם אהת. -ת.ת & 219 & $0.752292=33$ & 269 & 0.953345541 \\
\hline 20 & 0.00427780 & 70 & 0.15649541 & 120 & $(0.37212331$ & 170 & 0.58171423 & 2201 & $0.7 \times 6.347 .56$ & 270 & $0.957+364+2$ \\
\hline 21 & 0.00505495 & 71 & 0.16077108 & 121 & 0.376381 .31 & 171 & $0.585 .54 \times 06$ & 2221 & 0.79040151 & 271 & $(1.9: 111426614$ \\
\hline 22 & 0.00593668 & 72 & 0.1650 .5643 & 122 & $0.3 \times 063657$ & 172 & $0.5 \times 997999$ & $2: 2: 2$ & 0.7944 .7409 & $=272$ & $0.495 \div 41+71$ \\
\hline 23 & 0.00692804 & 73 & 0.16935049 & 123 & $0.384 \times 8 \times 51$ & 173 & $0 . .59+11008$ & 223 & 3יבי ה.0.798 & 273 & $0.9094+0199$ \\
\hline 24 & 0.00803316 & 74 & 0.17365240 & 124 & 0.38913732 & 174 & $(0.535-2383 \pi$ & $2: 94$ & $0.502 \sin 06$ & & \\
\hline 25 & 0.00925504 & 75 & 0.17796117 & 125 & $0.3933 \times 316$ & $17 \bar{i}$ & 0.60123647 78 & 2.25 & 0.806641 .352 & & \\
\hline 26 & 0.01059585 & 76 & 0.18227605 & 126 & 0.39762594 & $17 t i$ & 0.6064 .5431 & 226 & $0.8106 .50: 54$ & & \\
\hline 27 & 0.01205690 & 77 & 0.18659628 & 127 & 0.40186567 & 177 & 0.610612010 & 227 & $11 \times 1+69625$ & & \\
\hline 28 & 0.01363901 & 78 & 0.19092107 & 128 & $0.406 i 10242$ & $17 x$ & $0.61+73: 10$ & 2025 & (1.)1S740.59 & & \\
\hline 29 & 0.01534261 & 79 & 0.19524992 & 129 & 0.41033628 & 179 & 0.61855229 & $2: 9$ & $0.832-7836.4$ & & \\
\hline 30 & 0.01716768 & 80 & 0.19958212 & 130 & 0.41456709 & 180 & 0.62296972 & 230 & $0.526 \times 2531$ & & \\
\hline 31 & 0.01911363 & 81 & 0.20391714 & 131 & 0.41879507 & 181 & $0.627(1 \times 540)$ & 231 & $0.530 \times 66.5161$ & & \\
\hline 32 & 0.02117944 & 82 & 0.20825445 & 132 & 0.42302015 & 182 & 0.63119939 & 232 & 0.53490461 & & \\
\hline 33 & 0.02336343 & 83 & 0.21259344 & 133 & 0.42724233 & 18.3 & 0.63531164 & 2333 & $1 . \times 3 \times 44 \geq 2=4$ & & \\
\hline 34 & 0.02566335 & 84 & 0.21693388 & 134 & 0.43146169 & 1,84 & $0.639+12213$ & 234 & $0 . \$ 4297 \times 57$ & & \\
\hline 35 & 0.02807645 & 85 & 0.22127523 & 135 & 0.43567831 & 185 & 0.64353094 & 235 & 0.54701353 & & \\
\hline 36 & 0.03059953 & 86 & 0.22561712 & 136 & 0.43989210 & 186 & $0.64763 \times 07$ & 236 & 0.85104726 & & \\
\hline 37 & 0.03322916 & 87 & 0.22995916 & 137 & 0.44410322 & 187 & 0.65174352 & 237 & 0.85 .567963 & & \\
\hline 38 & 0.03596155 & 88 & 0.23430105 & 138 & 0.44831159 & 188 & $0.6 .5 \times 4730$ & $23 x$ & 0.8 .5911069 & & \\
\hline 39 & 0.03879305 & 89 & 0.23864248 & 139 & 0.45251730 & 189 & 0.65994947 & 239 & $0 . \times 68314046$ & & \\
\hline 40 & 0.04171968 & 90 & 0.24298315 & 140 & 0.45672033 & 190 & $0.664(04996$ & 240) & 0.86716894 & & \\
\hline 41 & 0.04473760 & 91 & 0.24732290 & 141 & 0.46092077 & 191 & 0.66814886 & 241 & 0.87119611 & & \\
\hline 42 & 0.04784292 & 92 & 0.25166128 & 142 & 0.46511861 & 192 & $0.6722+607$ & 242 & 0.57522199 & & \\
\hline 43 & 0.05103178 & 93 & 0.25599836 & 143 & 0.46931387 & 193 & 0.67634476 & $24: 3$ & 0.57924657 & & \\
\hline 44 & 0.05430036 & 94 & 0.26033369 & 144 & 0.47350660 & 194 & 0.680435577 & 244 & 0.883269994 & & \\
\hline 45 & 0.05764486 & 95 & 0.26466718 & 145 & 0.47769682 & $19 \pi$ & $0.684 .52 \times 25$ & 245 & 6.58729 .23011 & & \\
\hline 46 & 0.06106161 & 96 & $0.26 \times 99870$ & 146 & $0.4 \times 1 \times 8459$ & 196 & $0.6 \times 561913$ & 246 & $(1.59131269)$ & & \\
\hline 47 & 0.06454679 & 97 & 0.27332807 & 147 & 0.486 (069) K. & 197 & $0.69270 \times 4+1$ & 247 & 0.89533224 & & \\
\hline 48 & 0.06809690 & 98 & 0.2776 .5516 & 148 & 0.4902 .2744 & 198 & $0.696796 \quad 17$ & 248 & 1.8993501149 & & \\
\hline 49 & 0.07170835 & 99 & 0.28197988 & 149 & 0.49443319 & 199 & 0.700185232 & 249 & 0.9003366744 & & \\
\hline 50 & 0.07537756 & 100 & 0.28630201 & 150 & $0.49 \times 61135$ & 200 & $0.704 ! 96694$ & 2.50 & 0.901738309 & & \\
\hline
\end{tabular}

During fabrication it is recommended that the thermometer be evacuated while at about $450^{\circ} \mathrm{C}$ and then filled with dry gas and hermetically sealed. It is desirable to have oxygen present in the gas filling to ensure that trace impurities in the platinum will remain in an oxidised state. After completion, the

Tablc: 4. Values of $W_{\mathrm{CCr} \cdot \mathrm{es}}\left(T_{68}\right)$, according to the data given in Table 2, at the fixed-point temperatures

\begin{tabular}{|c|c|c|c|}
\hline Fixed point & $T_{6 \mathrm{~B}}(\mathrm{~K})$ & $t_{68}\left({ }^{\circ} \mathrm{C}\right)$ & $W_{c c t-68}$ \\
\hline e- $\mathrm{H}_{2}$ triple & 13.81 & .2 .59 .34 & 0.00141206 \\
\hline $\mathrm{e}-\mathrm{H}_{2} \quad 17.042$ & 17.042 & - 2.5ti.108 & 0.0025 .3444 \\
\hline ․ $\mathrm{H}_{2}^{2}$ boiling & 20.28 & -252.87 & $0.0(144 \times 517$ \\
\hline Ne hroiling & 27.102 & -246.048 & $(101221272$ \\
\hline () ${ }_{2}$ triple & 54.361 & -218.789 & 0.0919725 \\
\hline \multirow[t]{2}{*}{$\mathrm{O}_{2}$ bxiling } & $90.1 \times 8$ & $-1 \times 2.962$ & 0.24379909 \\
\hline & $27: 3.15$ & 0 & 1 \\
\hline $\mathrm{II}_{2} \mathrm{O}$ boiling & $37: 3.1 \pi$ & 100 & 1.399259668 \\
\hline in freezing & 50.5 .1181 & $2: 31.9681$ & 1.89257086 \\
\hline
\end{tabular}

resistanes element slould be stabilized by heating at a temperature higher than its intended maximum operating temperature and in any case not lower than $4.50 \%$

The insulation rexistance of the components supporting the resistance element and leads must be high cnough to avoid signiticant shunting of the clement. lior example, care must be taken to avoid condensation of water vapour between the leads at low temperatures, and intrinsic leakage in the insulators themselves at high temperatures. The insulators are usually fabricated from mica, silica or alumina, and these materials normally give adequate intrinsic insulation up to $500^{\circ} \mathrm{C}$. However, as the temperature approaches $630^{\circ} \mathrm{C}$, the problen becomes more critical and errors of $1 \mathrm{mK}$ or greater may easily occur. In the case of mica, there is the additional difficulty that significant amounts of water may be released during its exposure to temperitures above $450^{\circ} \mathrm{C}$, and unless 
this moisture is removed by periodic pumping or by a desiccant the insulation resistance will deteriorate rapidly.

To "nsure adequate stability in the resistance and the temperature coefficients of resistivity, the resistor of a stanclard platinum resistance thermometer should be maintained, as far as possible, in an annealed state. Added resistivity may arise both from the accidental cold working that results from normal thermometer handling and also as a result of rapid cooling when a thermometer is transferred rapidly from an environment above $500{ }^{\circ} \mathrm{C}$ : to room temperature. This latter increase in resistance is due to quenched-in, nonequilibrium concentrations of vacancy defects and is retained as long as the thermometer remains below 200 " Murk of the cold work and all of the quenched-in resistance may be removed by annealing at s(to) $(:$ for $30 \mathrm{~min}$.

Significant errors can be caused by radiation loss from the thermometer by totial reflection in the consiractional eomponents, particularly if these are of silica. Such loss in the sheath, but not in the internal

Table 5

Eliect of pressure on the freezing-point temperatures of metals

\begin{tabular}{|c|c|c|c|}
\hline \multirow[t]{2}{*}{ Metal } & \multirow{2}{*}{$\begin{array}{l}\text { Fretzing point } \\
\text { at } 1 \text { standard } \\
\text { atmosphere } \\
\text { (c) }\end{array}$} & \multicolumn{2}{|c|}{ P'ressure coefficient } \\
\hline & & $\begin{array}{l}\text { kel ins per } \\
\text { atmosphere }\end{array}$ & $\begin{array}{l}\text { kelrins per } \\
\text { centimetre } \\
\text { of liquid }\end{array}$ \\
\hline Mercury & -38.862 & +0.0054 & +0.000071 \\
\hline Indium & 156.634 & +0.0049 & $+0.000(0.33$ \\
\hline 'l'in & $2: 31.9681$ & +0.0083 .3 & +0.0000022 \\
\hline Bismuth & 271.442 & -0.0035 & -0.0000 .34 \\
\hline Cadmium & 321.108 & +0.0062 & +0.000048 \\
\hline Isend & 327.502 & +0.0080 & +0.000082 \\
\hline Zine & 419.58 & +0.0043 & +0.0000 .27 \\
\hline Antimoriy & 630.74 & +0.00085 & +0.000005 \\
\hline
\end{tabular}

components, can be suppressed by blackening the outer surface of the sheath (e.g., with a colloidal graphite suspension) or by sand-blasting the surface to produce a matt finish.

The completed thermometer should be tested to astablish that the depth of immersion is sufficient to avid heat ronduction errors. An effective way of doing this is to confirm that the apparent temperature rradient in a motal froezing point is in aurecment with that to be expected from hydrostatic effects (see T'able 5).

For temperatures below $90 \mathrm{~K}$ it is usual to use a smail platinum resistance thermometer, generally not larger than $5 \mathrm{~mm}$ in diameter and $60 \mathrm{~mm}$ in length, that can be totally immersed in a uniform temperature zone with heat conduction down the leads being supfressed by attaching them to a suitable guard ring. In orler to achieve good thermal contact between the resistor and its surroundings the resistor is contained in a thin sheath, commonly of platinum about $0.25 \mathrm{~mm}$ thick, which is filled with helium.

A useful criterion by which the efficiency of the annealing and the reliability of the thermometer may by judiged is the constancy of its resistance at a reference temperature. The temperatures of the triple point of water $(273.16 \mathrm{~K})$ and the boiling point of helium $(4.215 \mathrm{~K})$ are commonly used for this purpose. The first of these is convenient for most high tem- perature thermometers, while the second is not only often conveniently attained for thermometers built into eryogenic apparatus but has the additional advantage that the resistance is relatively insensitive. to temperature variations. In practice it is found that variations of resistance at the triple point of water for commercially produced high temperature thermometers should not exceed $4 \times 10^{-6} R\left(0^{\circ} \mathrm{C}\right)$ (equivalent to $\approx 1 \mathrm{mK}$ above $40 \mathrm{~K}$ ), and will not exceed $5 \times 10^{-7} R\left(0^{\circ} \mathrm{C}\right)$ over a reasonable periorl of use for the very best thermometers when these are handled with extreme care. For resistance thermometers used only at temperatures of $100^{\circ} \mathrm{C}$ or less, variations should not exceed $5 \times 10^{-7} R\left(0^{\circ} \mathrm{C}\right)$.

The small temperature rise of the thermometers caused by the measuring current may be determined by measurements at two currents.

\section{Standard Thermocouple}

satisfactory standard thermocouples have been made of wires of a uniform diameter between 0.35 and $0.65 \mathrm{~mm}$. The thermocouple wires must be thoroughly annealed in order to ensure constancy of e.m.f. in use. For this purpose it is necessary to heat the platinum wire to a temperature of at least $1100^{\circ} \mathrm{C}$ and the platinum-rhodium wire to $1450^{\circ} \mathrm{C}$. If the annealing is done before the wirs have been mounted in their insulators the completed thermocouple must bo heated again to a tomperature of at least $1100^{\circ} \mathrm{C}$ until its chertromotive foree has berin stabilized and locil inhomogeneities caused by strain have been removed. When this has been satisfactorily aceomplished the thermocouple e.m.f. should not be changed by chances in the temperature gradients along the wires; it should not change, for example, with increase of depth of immersion in an enclosure at a uniform temperature.

\section{Pressure}

In practice presiures are usually determined by means of a mercury columu. The mean density of pure mercury at the temperature $t_{68}$ in a barometric column supported by the pressure $p$ boing measured is given, with sufficient accuracy over the temperature range from $0^{\circ} \mathrm{C}$ to $40^{\circ} \mathrm{C}$ and for the pressures relevint to these measurements, by the relation

$$
\varrho\left(t_{68}, \frac{p}{2}\right)=\frac{\varrho\left(20^{\circ} \mathrm{C}, p_{0}\right)}{\left[1+A\left(t_{68}-20^{\circ} \mathrm{C}\right)+b^{\prime}\left(t_{68}-20^{\circ} \mathrm{C}\right)^{2}\right] \times\left(1-\chi\left(\frac{p}{\left.2^{-}-p_{0}\right)}\right) \mid\right.}
$$

where

$A=18115 \times 10^{-8^{\circ}} \mathrm{C}, 1, \quad B=0.8 \times 10^{-8^{\circ}} \mathrm{C}^{-2}$, $\chi=4 \times 10^{-11} \mathrm{~N}^{-1} \mathrm{~m}^{2}$,

$\varrho\left(20^{\circ}\left(, p_{0}\right)=13545.87 \mathrm{~kg} / \mathrm{m}^{3}\right.$ is the density of pure mercury at $t_{68}=20^{\circ} \mathrm{C}$ under a pressure $p_{0}=1$ standard atmosphere $\left(101325 \mathrm{~N} / \mathrm{m}^{2}\right)$.

A sufficiently accurate value of the local wravity may be obtained by using the Potsclam system and applying a correction of $-14 \times 10^{-5} \mathrm{~m} / \mathrm{s}^{2}(-14 \mathrm{milli}$ gals) ${ }^{6}$.

Hydrostatic head pressures within the fixed points

${ }^{6}$ By Resolution 1 (1968), the International Committee of Weights and Measures decided that for metrological purposes, the value of the acceleration due to gravity at Potsdam, the reference point of this System, should be taken as $9.81260 \mathrm{~m} / \mathrm{s}^{2}$ instead of $9.81274 \mathrm{~m} / \mathrm{s}^{2}$, the value adopted $\mathrm{m}$. tially. 
cells cause small but significant temperature effects; these are summarized in Table 5.

\section{Triple Point of Water}

The temperature of the triple point of water can be realized in sealed glass cells containing only water of high purity and of substantially the isotopic composition of ocean water. The cells have an axial well for the thermometers and the triple-point temperature is obtained wherever the ice is in equilibrium with a liquid-vapour surface. At a depth $h$ below the liquidvapour surface, the equilibrium temperature $t_{68}$ between ice and liquid water is given by

$$
t_{68}=A+B h
$$

where $A=0.01^{\circ} \mathrm{C}$ and $B=-7 \times 10^{-4} \mathrm{~m}^{-1}{ }^{\circ} \mathrm{C}$. The method recommended for preparing a triple-point cell consists of forming a thick layer of ice around the thermometer well by cooling from within, then melting enough of this sheath, also from within, to produce a new water-ice interface adjacent to the well. During the first hours following the preparation of the cell the temperature measured in the thermometer well rises fairly rapidly by a few ten thousandths of a kelvin becoming stable after from 1 to 3 days. This initial change of temperature is probably caused by the growth of the ice crystals or by the slow disappearance of strain in the crystals. A cell prepared in this way and kept in an ice bath is capable of maintaining a temperature constant to about $0.0001 \mathrm{~K}$ for several months. Even with cells from various sources, when used in this way, the differences in the temperatures obtained should in any case not exceed $0.0002 \mathrm{~K}$. A significant rise in temperature of the thermometer above the triple-point temperature may be caused by artificial light or sunlight falling on the ice-covered cell and it is therefore rocommended that measurements should be made with the cell suitably shielded from radiation.

Variations in the isotopic content of naturally occurring water are such that they will result in detectable differences in the triple-point temperature. Ocean water contains about 0.016 moles of deuterium, ${ }^{2} \mathrm{H}$, per 100 moles of hydrogen, ${ }^{1} \mathrm{H}$, and 0.04 moles of ${ }^{17} \mathrm{O}$ and 0.2 moles of ${ }^{18} \mathrm{O}$ per 100 moles of ${ }^{10} \mathrm{O}$. This proportion of heavy isotopes is substantially the highest to be found in naturally occurring water. Continental surface water normally contains about 0.015 moles of ${ }^{2} \mathrm{H}$ per 100 moles of ${ }^{1} \mathrm{H}$; water coming from polar snow may occasionally contain as little as 0.01 moles of ${ }^{2} \mathrm{H}$ per 100 moles of ${ }^{1} \mathrm{H}$.

The operation of purifying the water may slightly modify its isotopic composition and the isotopic composition at an ice-water interface is slightly dependent on the freezing technique.

An increase of 0.001 moles of ${ }^{2} \mathrm{H}$ per 100 moles of ${ }^{1} \mathrm{H}$ corresponds to an increase of temperature of the triple point of $0.00004 \mathrm{~K}$; this is the difference between the triple points for ocean water and the normally occurring continental surface water. The extreme difference in the triple-point temperatures of naturally occurring water is $0.00025 \mathrm{~K}$.

\section{Triple Point, $17.042 \mathrm{~K}$ Point and Boiling Point of Equilibrium Hydrogen}

Hydrogen has two molecular modifications, designated by the prefixes ortho and para, caused by different relative orientations of the two nuclear spins in the diatomic molecules. The equilibrium ortho-para composition is temperature dependent and at room temperature is about $75 \%$ orthohydrogen and $25 \%$ parahydrogen (so-called "normal hydrogen"). On liquefaction the composition changes slowly with time and there are corresponding changes in the physical properties. At the boiling point the equilibrium composition is $0.21 \%$ ortho- and $99.79 \%$ parahydro-, gen and the temperature is lower than that of normal hydrogen by about $0.12 \mathrm{~K}$. The name equilibrium hydrogen means in this document that the hydrogen has its equilibrium ortho-para composition at the relevant temperature. In order to avoid errors in the realization of these fixed points caused by indeterminate composition it is advisable to use equilibrium hydrogen converted by the use of a catalyst such as ferric hydroxide. Hydrogen of high chemical purity should be used as may be obtained by diffusion through palladium.

The temperature of equilibrium between solid, liquid and vapour phases of hydrogen can be realized by using a sufficient quantity of liquid hydrogen with some catalyst in a cavity in a copper block in which platinum resistance thermometers are immersed and which is surrounded by a vacuum space. The temperature of the block is reduced until the hydrogen is solidified and then the temperature is allowed to rise slowly and the transition at the triple point is observed. The flat portion of the time-temperature curve can be constant to $0.0001 \mathrm{~K}$ for $30 \mathrm{~min}$ or more.

The temperature of equilibrium between liquid and gaseous hydrogen is normally realized by the static method. In this method a cavity in a block of metal of high thermal conductivity is maintained at a temperature close to the boiling point by immersing it in liquid hydrogen. In order to avoid temperature gradients due to hydrostatic pressure the liquid hydro. gen makes contact with the top of the block only, the lower part being shiclded by a vacuum jacket. The cavity contains a small quantity of very pure liquid hydrogen together with some catalyst. The vapour pressure of this hydrogen is transmitted by a thin tube of low heat conduction connected to a manometer outside the enclosure. Precautions must be taken to avoid direct radiation down this tube into the cavity and to ensure that the tube at every point is at a higher temperature than the temperature at the surface of the liquid hydrogen in the cavity. Comparisons are made betwcen the vapour-pressure thermometer thus formed and platinum resistance thermometers im. mersed in closely fitting holes in the metal block adjacent to the cavity.

The validity of the measurements may be checked by showing that the values obtained are independent of the ratio of the rolume of the liquid hydrogen to the volume of the vapour in the cavity.

The temperature $T_{68}$ as a function of the vapour pressure of equilibrium hydrogen is given to an accuracy of a few millikelvins for the range from 13.81 $\mathrm{K}$ to $23 \mathrm{~K}$ by the equation

where

$$
\lg \frac{p}{p_{0}}=A+\frac{B}{T_{68}}+C T_{68}+D T_{68}^{2}
$$




$$
\begin{array}{llll}
A= & 1.711466, & B & -44.01046 \mathrm{~K} \\
C= & 0.0235909 \mathrm{~K}^{-1}, & I)= & -0.000048017 \mathrm{~K}^{-2} \\
P_{0} & 101325 \mathrm{~N} / \mathrm{m}^{2} . & &
\end{array}
$$

\section{Boiling Point of Neon}

The boiling point of neon can be realized in a manner similar to that described for hydrogen. The normal isotopic composition of neon is 0.0026 moles of ${ }^{21} \mathrm{Ne}$ and 0.088 moles of ${ }^{22} \mathrm{Ne}$ per 0.909 moles of ${ }^{20} \mathrm{Ne}$.

The temperature $T_{B S}$ as a function of the vapour pressure of neon is given to an accuracy of $+0.0002 \mathrm{~K}$ for the range from $27 \mathrm{~K}$ to $27.2 \mathrm{~K}$ by the equation

$$
\begin{aligned}
T_{68} & =\left[27.102+3.3144\left(\frac{p}{p_{0}}-1\right)-1.24\left(\frac{p}{p_{0}}-1\right)^{2}\right. \\
& \left.+0.74\left(\frac{p}{p_{0}}-1\right)^{3}\right] \mathrm{K} .
\end{aligned}
$$

\section{Triple Point and Boiling Point of Oxygen}

The triple point and the boiling point of oxygen can be realized in a manner similar to that described for hydrogen. Particular care must be given to the purity of the oxygen in the vapour-pressure thermometer. The $n$. ygen is sufficiently pure when the normal boiling point remains constant with the removal of successive fractions of vapour. The temperature $T_{68}$ as a function of the vapour pressure of oxygen is given to an accuracy of $\pm 0.0001 \mathrm{~K}$ for the range from $90.1 \mathrm{~K}$ to $90.3 \mathrm{~K}$ by the equation

$$
\begin{aligned}
T_{\text {Bs }} & =\left[90.1 \times 8+9.5648\left(\frac{p}{p_{0}}-1\right)-3.69\left(\frac{p}{p_{0}}-1\right)^{2}\right. \\
& \left.+2.2 .2\left(\frac{p}{p_{0}}-1\right)^{3}\right]_{\mathrm{K}} .
\end{aligned}
$$

\section{s. Boiling Point of Water}

The temperature of equilibrium between liquid water and its vapour is usually realized by the dynamic method with the thermometer in the saturated vapour. For precise calibration it is preferable to use closed systems in which the boiler and manometer are connected to a manostat filled with air or preferably helium.

The boiler must be constructed in such a way that contamination of the water is avoided. The thermometer must be protected from radiation emitted by bodies which are at temperatures different from the hoiling-point temperature. If the equilibrium temperature has been obtained the observed temperature (reduced to a constant pressure) will be independent of the time elapsed, variations in the rate of heat supplied to the liquid, and the depth of immersion of the thermometer.

A change in the proportion of deuterium in the water produces a change in the boiling point of water in the same direction as in the triple point but to about one third the extent

The temperature $t_{68}$ as a function of the vapour pressure of water is given, to an accuracy of $+0.0001 \mathrm{~K}$ for the range from $99.9^{\circ} \mathrm{C}$ to $100.1^{\circ} \mathrm{C}$ by the equation

$$
\begin{aligned}
t_{62} & =\left[100+28.0216\left(\frac{p}{p_{0}}-1\right)-11.642\left(\frac{p}{p_{0}}-1\right)^{2}\right. \\
& \left.=7.1\left(\frac{p}{p_{0}}-1\right)^{3}\right]^{\circ} \mathrm{C}
\end{aligned}
$$

\section{Freezing Points of Tin and Zinc}

Very reproducible temperatures can be realized by observing the flat part of the temperature versus time curve obtained during the slow freezing of very pure metals.

The melting and freezing of tin and zinc may be carried out in a crucible of very pure artificial graphite $(99.999 \%$ by weight) about $5 \mathrm{~cm}$ in diameter provided with an axial thermometer well. The depth of immersion of the thermometer in the metal must be sufficient to eliminate the influence of thermal conduction along the thermometer leads on the temperature of the sensitive element. It is convenient to contain the crucible and ingot of metal in a pyrex or silica tube under an inert atmosphere and to heat it in a metal-block furnace.

The aim of the cooling technique in the determination of the freezing point is to ensure that the thermometer sensor is as nearly as possible enclosed by, and is in thermal equilibrium with, a solid-liquid interface: shortly after nucleation there should be present either a completed solid shell nucleated on and thickening from the crucible wall or a completed solid mantle induced on the thermometer well.

The equilibrium temperature between solid and liquid metal varies slightly with pressure. The maynitudes of these variations are given in Table 5 .

High purity tin (99.9999\% by weight) supercools by $20 \mathrm{~K}$ to $30 \mathrm{~K}$ before solidification when cooled from the liquid state. The following technique has been successful for nucleating an ingot of tin while avoiding excessive undercooling of the freczing-point furnace. Starting with the temperature a few kelvins above the freezing point, the furnace is slowly cooled at about 0.1 kelvin per minute until the melt reaches its liciuiduspoint temperature; the sample holder containing the melt and a monitoring resistance thermometer is then either withdrawn into the throat of the furnace or removed from the furnace entirely. Under either of these eonditions the sample cools rapidly; when the rapid rise in temperature that indicates general nucleation is detected the sample holder is quichly replaced in the furnace which is still cooling slowly. Thereafter, as solidification proceeds slowly. a characteristic cooling curve for a high purity metal is realized having a temperature plateau that is reproducible for a particular sample to $\pm 0.0001 \mathrm{~K}$ for durations dependent on the rate of furnace cooling.

High purity zine (99.9999\% by weight) is treated in a somewhat different manner since it does not supercool excessively. A thin layer of solid metal is produced on the central thermometer well by removing the thermometer when the melt reaches the liquidus temperature, and either cooling the thermometer to room temperature and replacing it, or inserting a silica rod in its place for about $30 \mathrm{~s}$ before replacing the thermometer.

A criterion for ehecking that the purity of a sample of zinc or of tin is satisfactory is that its melting range is not more than $0.001 \mathrm{k}$.

\section{Freezing Points of Silver and lowd}

The equilibrium temperatures between the solid and liquid phases of silver and of gold may be realized in covered crucibles of either very pure artificial 
Table 6. Secondary reference points

\begin{tabular}{|c|c|}
\hline Equilibrium state & 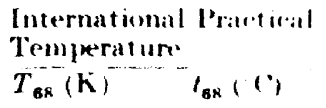 \\
\hline
\end{tabular}

Equilibrium between the solid, liquid and vapour phases of normal hydrogen (triple point of normal hydrogen)

Equilibrium between the liquid and vapour phases of normal hydrogen (boiling point of normal hydrogen)

$$
\lg \frac{p}{p_{0}}=A+\frac{B}{T_{68}}+C T_{68}+1 T_{\mathrm{E8}}^{2}
$$

$A-1.734791, \quad B=-44.62368 \mathrm{~K}, \quad C=0.0231869 \mathrm{~K}^{-1}, \quad D=-0.000048017 \mathrm{~K}^{-2}$

for the temperature range from $13.956 \mathrm{~K}$ to $30 \mathrm{~K}$.

Equilibrium between the solid, liquid and vapour phases of neon (triple point of neon)

Equilibrium between the liquid and vapour phases of neon

$$
\lg \frac{p}{p_{0}}=A+\frac{B}{T_{68}}+C T_{68}+D T_{68}^{2}
$$

$A=4.61152, \quad B=-106.3851 \mathrm{~K}, \quad C=-0.0368331 \mathrm{~K}^{-1}, \quad D=4.24892 \div 10^{-4} \mathrm{~K}^{-2}$

for the temperature range from $24.555 \mathrm{~K}$ to $40 \mathrm{~K}$.

Equilibrium between the solid, liquid and vapour phases of nitrogen (triple point of nitrogen) Equilibrium between the liquid and vapour phases of nitrogen (boiling point of nitrogen)

$$
\lg \frac{p}{p_{0}}=A+\frac{B}{T_{68}}+C \lg \frac{T_{68}}{T_{0}}+D T_{68}+E T_{68}^{2}
$$

$A=5.893139, \quad B--404.13105 \mathrm{~K}, \quad C=-2.3749, \quad J-0.0142505 \mathrm{~K}^{-1}$

$E=72.5342 \times 10^{-6} \mathrm{~K}^{-2}$

for the temperature range from $63.148 \mathrm{~K}$ to $84 \mathrm{~K}$.

Equilibrium between the liquid and vapour phases of oxygen

$$
\lg \frac{p}{p_{0}}=A+\frac{B}{T_{68}}+C \lg \frac{T_{68}}{T_{0}}+I J T_{68}+E T_{68}^{2}
$$

$A=5.961546, \quad B--467.45576 \mathrm{~K} . \quad C=-1.664512 . \quad D=-0.01321301 \mathrm{~K}^{-1}$,

$E=50.8041 \times 10^{-6} \mathrm{~K}^{-2}$

for the temperature range from $54.361 \mathrm{~K}$ to $94 \mathrm{~K}$.

Equilibrium between the solid and vapour phases of carbon dioxide (sublimation point of carbon dioxide)

$$
T_{68}=\left[194.674+12.264\left(\frac{p}{p_{0}}-1\right)-9.15\left(\frac{p}{p_{0}}-1\right)^{2}\right] \mathrm{K}
$$

for the temperature range from $194 \mathrm{~K}$ to $195 \mathrm{~K}$.

Equilibrium between the solid and liquid phases of mercury (freezing point of mercury)*

Equilibrium between ice and air-saturated water (ice point)

Equilibrium between the solid, liquid and vapour phases of phenoxybenzene (diphenyl ether) (triple point of phenoxybenzene)

Equilibrium between the solid, liquid and vapour phases of benzoic acid (triple point of benzoic acid)

Equilibrium between the solid and liquid phases of indium (freezing point of indium)a

Equilibrium between the solid and liquid phases of bismuth (freezing point of bismuth) ${ }^{\mathrm{a}}$

Equilibrium between the solid and liquid phases of cadmium (freezing point of cadmium)

Equilibrium between the solid and liquid phases of lead (freezing point of lead) ${ }^{3}$

Equilibrium between the liquid and vapour phases of mercury (boiling point of mercury)

$$
t_{68}=\left[356.66+55.552\left(\frac{p}{p_{0}}-1\right)-23.03\left(\frac{p}{p_{0}}-1\right)^{2}+14.0\left(\frac{p}{p_{0}}-1\right)^{3}\right]{ }^{\circ} \mathrm{C}
$$

for $p=90 \times 10^{3} \mathrm{~N} / \mathrm{m}^{2}$ to $104 \times 10^{3} \mathrm{~N} / \mathrm{m}^{2}$.

Equilibrium between the liquid and vapour phases of sulphur (boiling point of sulphur)

$$
t_{\mathrm{B8}}=\left[444.674+69.010\left(\frac{p}{p_{0}}-1\right) \cdot 27.48\left(\frac{p}{p_{0}}-1\right)^{2}+19.14\left(\frac{p}{p_{0}}-1\right)^{3}\right]{ }^{\circ} \mathrm{C}
$$

717.824

444.674

for $p-90 \times 10^{3} \mathrm{~N} / \mathrm{m}^{2}$ to $104 \times 10^{3} \mathrm{~N} / \mathrm{m}^{2}$.

Equilibrium between the solid and liquid phases of the copper-aluminium eutectic

Equilibrium between the solid and liquid phases of antimony (freezing point of antimony)

Equilibrium between the solid and liquid phases of aluminium (freezing point of aluminium)

Equilibrium between the solid and liquid phases of copper (freezing point of copper)

Equilibrium between the solid and liquid phases of nickel (freezing point of nickel)

Equilibrium between the solid and liquid phases of cobalt (freezing point of cobalt)

Equilibrium between the solid and liquid phases of palladium (freezing point of palladium)

Equilibrium between the solid and liquid phases of platinum (freezing point of platinum)

Equilibrinm between the solid and liquid phases of rhodium (freezing point of rhodium)

Equilibrium between the solid and liquid phases of iridium (freezing point of iridium)

Equilibrium between the solid and liquid phases of tungsten (temperature of melting tungsten)

- See Table 5 for the effect of pressure variations on these freezing points. 
graphite, or ceramic material or vitreous silica. If graphite is aserl it is advisable to prevent the access of nir to the comible so that the graphite is not oxidised. Molten silver whould be protected so as to prevent the solulium ol oxygen into it and the consequent deprexsion of tho freering point.

The ingot of metal should be heated to a uniform temperature a few kelvins above the melting point of the metal and then cooled slowly. The thermocouple to be calibrated, mounted in a protecting tube of suitable refractory material, with refractory insulators separating the two wires, is immersed in the molten metal which is then allowed to freeze. The depth of immersion of the thermocouple in the metal must be sufficient to eliminate the influence of thermal conduction along the wires of the thermocouple.

That the equilibrium temperature is obtained can be checked by the following criteria: the electromotive force of the thermocouple should be independent of small variations in the depth of immersion in the ingot of metal during successive freezes, and should remain constant for at least $5 \mathrm{~min}$ during one freeze.

A blackbody at the freezing point of gold is required for the establishment of the reference temperature for radiation temperature measurements. For the realization of "rch a blackbody the crucible containing the gold murt be modified to provide a uniform temperature enclosure immersed in the gold. A blackbody enclosure is more readily achieved if the material employed for its construction has already a high emissivity and therefore graphite is very suitable for this purposè.

\section{Secondary Reference Points}

As well as the defining fixed points of the IPTS-68 given in Table 1 other reference points are available. Some of these points and their temperatures on the IPTS-68 are given in Table 6. Except for the triple points and the vapour pressure-temperature equations each temperature is that for a system in equilibrium under the pressure of one standard atmosphere.

\section{Appendix I.}

\section{History of the Development} of the International Temperature Seales; Differences between the IPTS-68 and the IPTS-48

The International Temperature Scale was adopted in 1927 to overcome the practical difficulties of the direct realization of thermodynamic temperatures by gas thermometry and to unify the existing national temperature scales. It was introduced by the 7 th General Conference of Weights and Measures with the intention of providing a practical scale of temperature which was easily and accurately reproducible and which gave as nearly as possible thermodynamic temperatures.

The International Temperature Scale was revised in 1948. The experimental procedures by which the Scale was roalized remained substantially unchanged, but two amendments were made to the definition of the Scale resulting in appreciable changes in the numerical values assigned to measured temperatures. The change in the value of the temperature of the freezing point of silver from $960.5^{\circ} \mathrm{C}$ to $960.8^{\circ} \mathrm{C}$ changed temperatures measured with the stindind thermocouple (range $630^{\circ} \mathrm{C}$ to $1063^{\circ} \mathrm{C}$ ); the maximum difference was about $0.4 \mathrm{~K}$ near $800^{\circ} \mathrm{C}$. The adoption of the value of 0.01438 metre kelvin instead of $0.014 \% 32$ metre kelvin for the radiation constant $c_{2}$ changed all tomperatures above the freezing point of gold, while the use of the Planck radiation formula instead of the Wien formula affected the very high temperatures. Temperatures above the freezing point of gold were decreased, for example, by $2.2 \mathrm{~K}$ at $1500^{\circ} \mathrm{C}$ and by $6 \mathrm{~K}$ at $2000^{\circ} \mathrm{C}$. Also at this revision, in order to secure international uniformity of nomenclature, the 9 th General Conference of Weights and Measures decided to abandon the word "Centigrade" and its French equivalent "Centésimal" in favour of the name "Celsius". That is "C" was now regarded as the abbreviation of "degree Celsius".

Table 7. Estimated uncertainties of the assigned values of the defining facd points in terms of thermodynamic tempercetures

$\begin{array}{lll}\text { Defining fixed point } & \text { Assigned } & \text { Estimated } \\ \text { value } & \text { uncertainty }\end{array}$

(K)

Triple point of equilibrium hýdrogen

$17.042 \mathrm{~K}$ point

Boiling point of equilibrium hydrogen

Boiling point of neon

Triple point of oxygen

Boiling point of oxygen

Triple point of water

Boiling point of water

Freezing point of tin

Freezing point of zinc

Froezing point of silver

Freezing point of gold

$\begin{array}{ll}13.81 \mathrm{~K} & 0.01 \\ 17.042 \mathrm{~K} & 0.01 \\ 20.28 \mathrm{~K} & 0.01 \\ 27.102 \mathrm{~K} & 0.01 \\ 54.361 \mathrm{~K} & 0.01 \\ 90.188 \mathrm{~K} & 0.01 \\ 273.16 \mathrm{~K} & \text { lixact by } \\ & \text { definition } \\ 100{ }^{\circ} \mathrm{C} & 0.005 \\ 231.9681{ }^{\circ} \mathrm{C} & 0.015 \\ 419.58^{\circ} \mathrm{C} & 0.03 \\ 961.93{ }^{\circ} \mathrm{C} & 0.2 \\ 1064.43^{\circ} \mathrm{C} & 0.2\end{array}$

An amended edition of the 1948 Scale was adopted by the 11th General Conference of Weights and Measures under the new title "International Practical Temperature Scale of 1948 (amended edition of 1960 )", the numerical values of temperature remaining the same as in 1948. The new edition incorporated the new definition of the kelvin given by defining the thermodynamic temperature of the triple point of water as exactly $273.16 \mathrm{~K}$ (10th General Conference of Weights and Measures 1954, Resolution 3). It was also recognized at this time that the IPTS no longer represented the thermodynamic temperature as closely as possible and the text included a section on the differences between them. The IPTS-68 has been devised to bring these differences within the limits of accuracy to which the thermodynamic temperatures are known (see 'Table 7) and to extend the Sonle to lower temperatures.

The IP'IS-68 differs from the IPTS-4S in the following ways. The lower limit of the Scale is now 13.81 $\mathrm{K}$ instead of $90.18 \mathrm{~K}$. The values assigned to the defining fixed points are modified where necessary to conform as nearly as possible to the thermodynamic temperatures, the only points remaining unchanged being the triple point of water, which is permanently fixed by definition, and the boiling point of water. The interpolation instruments remain the same as before but the standard platinum resistance thermometicr is 
Table 8

A proximate differences $\left(t_{68}-t_{5 \times}\right)$. in kelvins, between the values of temperature given by the IP'TS' of 1968 and the IPT'N of 1!948

\begin{tabular}{|c|c|c|c|c|c|c|c|c|c|c|c|}
\hline$t_{6 \mathrm{H}} \cdot \mathrm{C}$ & 0 & -10 & -20 & -30 & -40 & -50 & -60 & $\ldots 70$ & 80 & -90 & 100 \\
\hline $\begin{array}{r}-100 \\
-\quad 0\end{array}$ & $\begin{array}{l}0.022 \\
0.000\end{array}$ & $\begin{array}{l}0.013 \\
0.006\end{array}$ & $\begin{array}{l}0.003 \\
0.012\end{array}$ & $\begin{array}{r}-0.006 \\
0.018\end{array}$ & $\begin{array}{r}-0.013 \\
0.024\end{array}$ & $\begin{array}{r}-0.013 \\
0.029\end{array}$ & $\begin{array}{r}-0.005 \\
0.032\end{array}$ & $\begin{array}{l}0.007 \\
0.034\end{array}$ & $\begin{array}{l}0.012 \\
0.033\end{array}$ & 0.029 & 0.02 .2 \\
\hline$t_{68}{ }^{\circ} \mathrm{C}$ & 0 & 10 & 20 & 30 & 40 & 50 & 60 & 70 & 80 & 90 & 160 \\
\hline $\begin{array}{r}0 \\
100\end{array}$ & $\begin{array}{l}0.000 \\
0.000\end{array}$ & $\begin{array}{r}-0.004 \\
0.004\end{array}$ & $\begin{array}{r}-0.007 \\
0.007\end{array}$ & $\begin{array}{r}0.009 \\
0.012\end{array}$ & $\begin{array}{r}-0.010 \\
0.016\end{array}$ & $\begin{array}{r}-0.010 \\
0.020\end{array}$ & $\begin{array}{r}-0.010 \\
0.025\end{array}$ & $\begin{array}{r}0.008 \\
0.029\end{array}$ & $\begin{array}{r}-0.0016 \\
0.034\end{array}$ & $\begin{array}{l}0.01013 \\
0.03858\end{array}$ & $\begin{array}{l}0 .(6) 101 \\
0.6403\end{array}$ \\
\hline 200 & 0.043 & 0.047 & 0.051 & 0.054 & 0.058 & 0.061 & 0.064 & 0.067 & 0.069 & 0.071 & 0.073 \\
\hline 300 & 0.073 & 0.074 & $0.07 \%$ & 0.076 & 0.077 & 0.077 & 0.077 & 0.077 & 0.077 & 0.076 & 0.076 \\
\hline 400 & 0.076 & 0.075 & 0.075 & 0.075 & 0.074 & 0.074 & 0.074 & 0.075 & 0.076 & 0.077 & 0.079 \\
\hline 500 & 0.079 & 0.082 & 0.085 & 0.089 & 0.094 & 0.100 & 0.108 & 0.116 & 0.126 & 0.137 & 0.150 \\
\hline 600 & 0.150 & 0.165 & 0.182 & 0.200 & 0.23 & 0.25 & 0.28 & 0.31 & 0.34 & 0.36 & 0.39 \\
\hline 700 & 0.39 & 0.42 & 0.45 & 0.47 & 0.50 & 0.53 & 10.56 & אד. 0. & 0.61 & 0.644 & 0.67 \\
\hline 800 & 0.67 & 0.70 & 0.72 & 0.75 & 0.78 & 0.81 & 0.84 & 0.87 & 0.89 & 0.92 & 0.95 \\
\hline 900 & 0.95 & 0.98 & 1,01 & 1.04 & 1.07 & 1.10 & 1.12 & 1.15 & 1.18 & 1.21 & 1.24 \\
\hline 1000 & 1.24 & 1.27 & 1.30 & 1.33 & 1.36 & 1.39 & 1.42 & 1.44 & & & \\
\hline$t_{68}{ }^{\circ} \mathrm{C}$ & 0 & 100 & 200 & 300 & 400 & 500 & 600 & 700 & 800 & $9(4)$ & $10(x)$ \\
\hline 1000 & & 1.5 & 1.7 & 1.8 & 2.0 & 2.2 & 2.4 & 2.6 & 2.8 & 3.0 & 3.2 \\
\hline 2000 & 3.2 & 3.5 & 3.7 & 4.0 & 4.2 & 4.5 & 4.8 & 5.0 & 5.3 & . 6 & 5.9 \\
\hline 3000 & 5.9 & 6.2 & 6.5 & 6.9 & 7.2 & 7.5 & 7.9 & 8.2 & 8.6 & 9.0 & 9.3 \\
\hline
\end{tabular}

now required to have $W\left(100^{\circ} \mathrm{C}\right)$ at least equal to 1.3925 instead of 1.3920 . Over the temperature range from $90.188 \mathrm{~K}$ to $273.15 \mathrm{~K}$ the Callendar-Van Dusen equation is no longer used for interpolation, instead the reference function $W_{\text {CCT-68 }}\left(T_{68}\right)$ is employed. Above $0{ }^{\circ} \mathrm{C}$, the Callendar equation is modified so that interpolated values of temperature conform more nearly with thermodynamic-temperature values. Final$l_{y}$ the latest value of $c_{2}$, namely 0.014388 metre kelvin is introduced in the Planck equation for determining temperatures above the freezing point of gold. The effect of all these changes is summarised in Table 8 which gives the differences between the values of temperature derived from the IPTS-68 and the IPTS48.

In the range from $13.81 \mathrm{~K}$ to $90.188 \mathrm{~K}$ the IPTS-68 is based on the average of four "national scales" and on chosen "best" temperatures for the defining fixed points. These national scales are each defined in terms of platinum resistance thermometers calibrated against a gas thermometer and are highly reproducible.

The differences between the IPTS- 68 and the national scales are published in Metrologia, Vol. 5, p. 47. (1969). This allows the use of the national scales and these differences to give a close approximation to the IIrIs-tis.

The text of this definition of the IP'TS-6s eonlome with the decision of the 13th General conference of Weighte and Mensures to denote the unit of thermo. "fynmic tempernture by the name "kelvin". symbol K. mel to denote a temperature interval by the same unit. and symbol or by "degree (elsius" of "."(".".
A ppendix II.

Practical scales of Temperature

for Ise over the Range from $0.2 \mathrm{~K}$ to $5.2 \mathrm{~K}$

Temperatures can be derived from neasured rapour pressures of ${ }^{4} \mathrm{He}$ and ${ }^{3} \mathrm{He}$. The upper limits for use are set by the critical points of the gases (5.2 K for ${ }^{4} \mathrm{He}$ and $3.3 \mathrm{~K}$ for ${ }^{3} \mathrm{He}$ ) and the lower limit by the vapour pressures becoming too low for practical measurement. The "1958 "He scale" and the "196:- "He scale" are the recommended sales in which temperatures are denoted by $T_{58}$ and $T_{62}$ respectively

The "1958 ${ }^{4} \mathrm{He}$ scale", recommended in 1950 by the International Committee of Weights and Measures. is defined by a table of ${ }^{4} \mathrm{He}$ vapour pressures versus temperature (Comité Consultatif de. Thermometrie. 5e session 1958, p. T 192 and Procis-Verbaux ('IPll. $26-\mathrm{A}, 1958$, p. T 192$)^{\mathrm{\gamma}}$

The "1962 "He scale", recommended by the International Committee of Weights and Measures in $196: 2$. is defined by an equation giving the vapour pressure of ${ }^{3} \mathrm{He}$ as a function of temperature ('omité consultatif de Thermométrie, $6^{\mathrm{e}}$ session $\left.1962, \mathrm{p} .1 \times 4\right)^{9}$.

In the temperature range between $0.9 \mathrm{~K}$ and the critical temperature of ${ }^{3} \mathrm{He}$ the temperatures $T_{58}$ and $T_{62}$ are believed to be in ayreement to within $0.3 \mathrm{mK}$.

? Recent measurements by the aroustic thermometer give temperatures higher than those of the He vapour pressure seales: the difference at the boiling point of ${ }^{4} \mathrm{He}$ is about $0.00 \mathrm{~h}$.

"An cxpanded form of this table together with anxiliary information is given in the J. Res. Nat. Bur. Standards 64.1.1 (1960).

- A table of values and information on measuring vapour pressures are given in the J. Res. Nat. Bur. Niandards bs 1 . $547,559,567,579(19 t 64)$.

Ofprint from , Metrologia"

Journal International de Métrologie scientifique - International Journal of scientific Metrology Internationale Zeitschrift für wissenschaftliche Metrologie. Vol. 5, No. 2, A pril 1969, Pages 3;)-4t 


\section{APPENDIX B. Comparison of Assigned Fixed Point Values and Interpolating Formulae in the Temperature Ranges Defined by the Platinum Resistance Thermometer for ITS-27, ITS-48, IPTS-48, and IPTS-68}

(a) Comparison of fixed point values

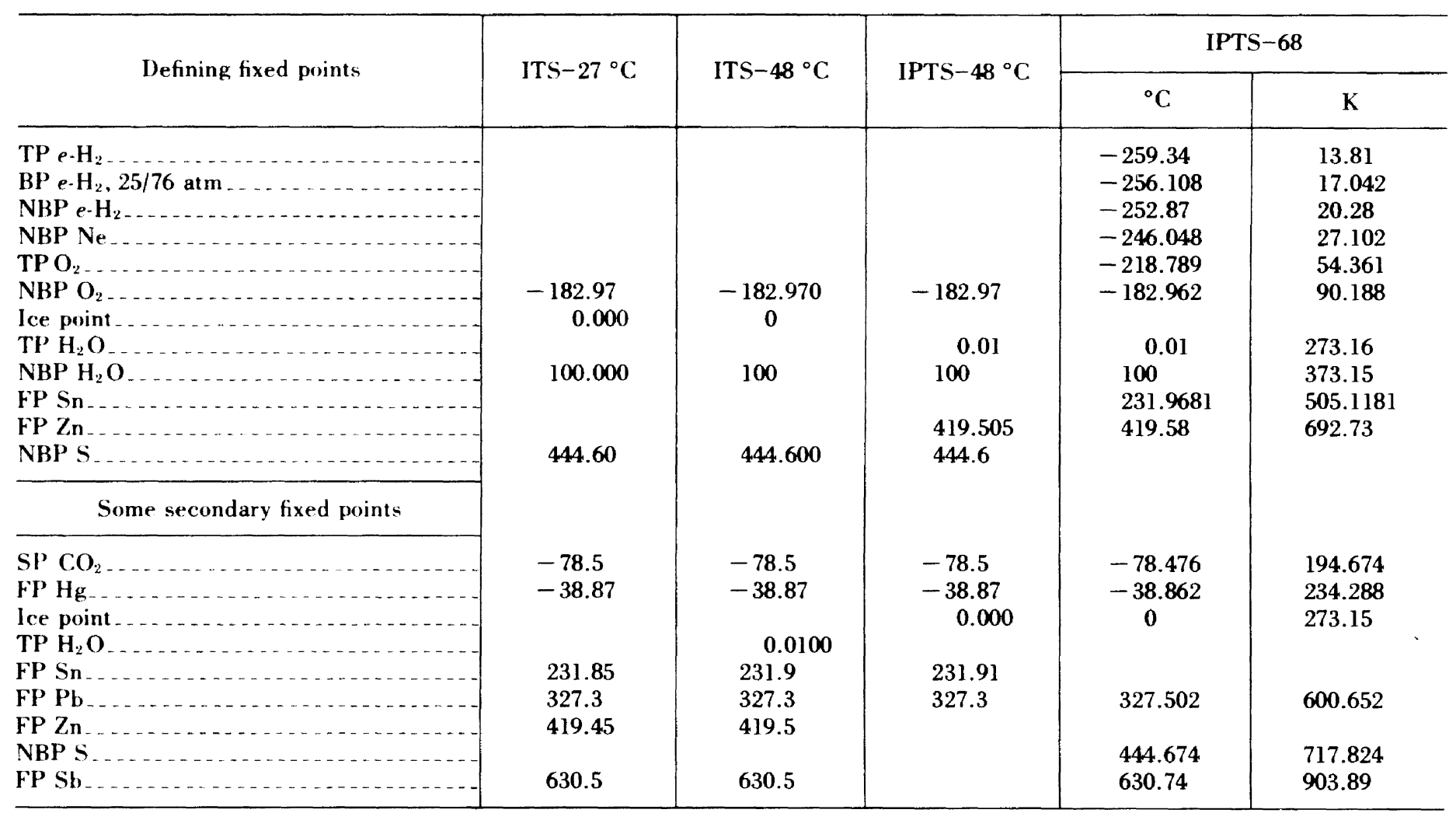

$\mathrm{TP}=$ triple point, $\mathrm{BP}=$ boiling point, $\mathrm{NBP}=$ normal boiling point, $\mathrm{FP}=$ freezing point, $\mathrm{SP}=$ sublimation point 


\section{APPENDIX B. Comparison of Assigned Fixed Point Values and Interpolating Formulae in the Temperature Ranges Defined by the Platinum Resistance Thermometer for ITS-27, ITS-48, IPTS-48, and IPTS-68-Continued}

\begin{tabular}{|c|c|c|c|c|}
\hline & ITS -27 & ITS -48 & IPTS-48 & IPTS-68 \\
\hline$R(100) / R(0)$ of $\mathrm{Pt}$ & $\geqq 1.390$ & $>1.3910$ & $\geqq 1.3920$ & $\geqq 1.3925$ \\
\hline Temperature Range & -190 to $0^{\circ} \mathrm{C}$ & -182.970 to $0^{\circ} \mathrm{C}$ & -182.97 to $0^{\circ} \mathrm{C}$ & 13.81 to $273.15 \mathrm{~K}$ \\
\hline Interpolating Formula -- & ${ }^{a}$ quartic & a quartic & ${ }^{a}$ quartic & $\begin{array}{l}\text { reference function } \\
\text { plus deviation } \\
\text { d polynomials }\end{array}$ \\
\hline Calibration Temperatures. & $\begin{array}{l}\mathrm{NBP} \mathrm{O}_{2}, \text { ice point } \\
\quad \mathrm{NBP} \mathrm{H}_{2} \mathrm{O}, \& \mathrm{NBP} \mathrm{S}\end{array}$ & $\begin{array}{l}\mathrm{NBP} \mathrm{O}_{2} \text {, ice point, } \\
\quad \mathrm{NBP} \mathrm{H}_{2} \mathrm{O}, \& \mathrm{NBP} \mathrm{S}\end{array}$ & $\begin{array}{l}\mathrm{NBP} \mathrm{O}_{2}, \mathrm{TP} \mathrm{H}_{2} \mathrm{O} \\
\mathrm{NBP} \mathrm{H}_{2} \mathrm{O}, \& \text { NBP S } \\
\text { or } \mathrm{FP} \mathrm{Zn}\end{array}$ & $\begin{array}{l}\mathrm{TP} e-\mathrm{H}_{2}, \mathrm{BP} e-\mathrm{H}_{2} \\
(25 / 76 \text { atm }), \mathrm{NBP} \\
e \cdot \mathrm{H}_{2}, \mathrm{NBP} \mathrm{Ne} \\
\mathrm{TPO}_{2}, \mathrm{NBP} \mathrm{O}_{2}, \\
\mathrm{TP} \mathrm{H}_{2} \mathrm{O}, \mathrm{NBP} \mathrm{H}_{2} \mathrm{O} \\
\text { or FP Sn, \& FP Zn. }\end{array}$ \\
\hline Temperature Range & 0 to $660^{\circ} \mathrm{C}$ & 0 to $630.5^{\circ} \mathrm{C}$ & 0 to $630.5^{\circ} \mathrm{C}$ & 0 to $630.74^{\circ} \mathrm{C}$ \\
\hline Interpolating Formula & ${ }^{b}$ quadratic & ${ }^{b}$ quadratic & ${ }^{b}$ quadratic & $\begin{array}{l}\text { Quadratic plus e cor- } \\
\text { rection function }\end{array}$ \\
\hline Calibration Temperatures.. & $\begin{array}{l}\text { ice point, } \mathrm{NBP} \mathrm{H}_{2} \mathrm{O} \\
\text { \& NBP S }\end{array}$ & $\begin{array}{l}\text { ice point, } \mathrm{NBP} \mathrm{H}_{2} \mathrm{O} \text {, } \\
\quad \text { \& NBP S }\end{array}$ & $\begin{array}{l}\mathrm{TP} \mathrm{H}_{2} \mathrm{O}, \mathrm{NBP} \mathrm{H}_{2} \mathrm{O} \\
\quad \& \mathrm{NBP} \mathrm{S}\end{array}$ & $\begin{array}{l}\mathrm{TP} \mathrm{H}_{2} \mathrm{O}, \mathrm{NBP} \mathrm{H}_{2} \mathrm{O} \\
\quad \text { or FP } \mathrm{Sn}, \& \mathrm{FP} \mathrm{Zn} .\end{array}$ \\
\hline
\end{tabular}

a Quartic interpolation formula: $R(t)=R(0)\left[1+A t+B t^{2}+C(t-100) t^{3}\right]$.

${ }^{b}$ Quadratic interpolation formula: $R(t)=R(0)\left(1+A t+B t^{2}\right)$.

'Reference function

$$
T_{68}=\sum_{i=0}^{20} A_{i}\left[\ln W^{*}(T)\right]^{i} \quad\left(\text { Values of } A_{i}\right. \text { are given in Appendix A.) }
$$

d Deviation polynomials: $\quad \Delta W(T)=W(T)-W^{*}(T)$

13.81 to $20.28 \mathrm{~K}: \quad \Delta W(T)=A_{1}+B_{1} T+C_{1} T^{2}+D_{1} T^{3}$

20.28 to $54.361 \mathrm{~K}: \quad \Delta W(T)=A_{2}+B_{2} T+C_{2} T^{2}+D_{2} T^{3}$

54.361 to $90.188 \mathrm{~K}: \quad \Delta W(T)=A_{3}+B_{3} T+C_{3} T^{2}$

-182.962 to $0^{\circ} \mathrm{C}: \quad \Delta W(t)=A_{4} t+C_{4} t^{3}\left(t-100^{\circ} \mathrm{C}\right)$

where $T=T_{68}, t=t_{68}, W^{*}=W_{\mathrm{CCT}-68}$.

e Quadratic interpolation formula plus correction function: $R\left(t^{\prime}\right) / R(0)=1+A t^{\prime}+B t^{\prime 2}$ plus

$$
t_{68}=t^{\prime}+0.045\left(\frac{t^{\prime}}{100{ }^{\circ} \mathrm{C}}\right)\left(\frac{t^{\prime}}{100^{\circ} \mathrm{C}}-1\right)\left(\frac{t^{\prime}}{419.58{ }^{\circ} \mathrm{C}}-1\right)\left(\frac{t^{\prime}}{630.74{ }^{\circ} \mathrm{C}}-1\right) .
$$




\section{APPENDIX C. Tables of Difference in the Values of Temperature Between the IPTS-48 and IPTS-68 and Between the NBS-1955 and IPTS-68 in the Temperature Range $13 \mathrm{~K}$ to $630{ }^{\circ} \mathrm{C}$}

The values given are the calculated differences between values of temperature on the IPTS -48 and on the IPTS-68, and, below the oxygen point, between values on the NBS-55 scale and on the NBS-IPTS-68 (see sec. 7.7). The calculations above the oxygen point are based upon the differences of the scales as defined in their respective texts (see Appendix A). The assumption is made that the value of a temperature on each scale is uniquely defined by its text. (In contrast, refer to the last paragraph of sec. 2.2.) The further assumption is made that, on the IPTS-48, the value $419.505{ }^{\circ} \mathrm{C}$ recommended by the text of the IPTS -48 for the freezing point of zinc is exactly correct. The coefficients employed were $\alpha=0.0039259665$, $\delta=1.491356$, and $\beta=0.1109843$ on the IPTS -48 . These values were derived from the reference function $\left(W^{*}\right)$ and the corresponding values of $\alpha$ and $\delta$ given in the text of the IPTS-68 (see Appendix A).

The calculation of the differences between values on the NBS -55 scale and the IPTS -68 are based upon the estimated differences published by Bedford, Durieux, Muijwijk, and Barber (Metrologia 5, 47 (1969)). The calibration of a thermometer on the NBS-55 scale was first adjusted by these published differences. The difference $(\Delta W)$ between each of these adjusted values and the corresponding value of the reference function $\left(W^{*}\right)$ was then smoothed by a least squares method using the nearest five points. These smoothed values were added to the reference function values and the resulting table subtabulated at $0.1 \mathrm{~K}$ intervals by cubic interpolation. The differences between these results and the calibration on the NBS-55 scale are tabulated here as the estimated differences between the NBS-55 scale and the IPTS -68 .

The two scales formerly used, i.e., the IPTS -48 and the NBS-55 scale, as realized and maintained at the National Bureau of Standards, were continuous through their point of joining, the normal boiling point of oxygen. Bedford, et al. (loc. cit.) however, estimated that the "oxygen point" upon which these scales were based was too hot by $0.0019 \mathrm{~K}$. The correction to be applied to values on the NBS-55 scale to get values on the NBS-IPTS68 scale is, as previously stated, based on the estimates of Bedford, et al. and, therefore, includes a correction for their estimated error in the hotness of the NBS "oxygen point." In contrast, the cor rections to be used to adjust values on the IPTS -48 to values on the IPTS -68 are based only upon the differences in the definitions of the two scales. This tacitly assumes that all fixed points are perfectly realized. The result of using the different bases for the temperature differences in the two ranges (above and below the oxygen point) is a discontinuity in the estimated corrections at the oxygen point. 
The Corrections to Values of Temperature on the NBS-55 Scale. (The correction $\left(T_{68}-T_{\text {NBS-55 }}\right)$ is given with the argument in $\boldsymbol{T}_{\mathrm{NBS}-55^{\circ}}$ )

\begin{tabular}{|c|c|c|c|c|c|c|c|}
\hline $\begin{array}{l}T \\
\mathbf{K}\end{array}$ & $\begin{array}{c}\text { Corr. } \\
\text { mK }\end{array}$ & $\begin{array}{l}T \\
\mathbf{K}\end{array}$ & $\begin{array}{c}\text { Corr. } \\
\mathbf{m K}\end{array}$ & $\begin{array}{l}T \\
\mathrm{~K}\end{array}$ & $\begin{array}{c}\text { Corr. } \\
\text { mK }\end{array}$ & $\begin{array}{l}T \\
\mathbf{K}\end{array}$ & $\begin{array}{c}\text { Corr. } \\
\text { mK }\end{array}$ \\
\hline & & 15.0 & 2.04 & 20.0 & 8.94 & 25.0 & 7.76 \\
\hline & & 15.1 & 2.36 & 20.1 & 8.96 & 25.1 & 7.71 \\
\hline & & 15.2 & 2.61 & 20.2 & 8.95 & 25.2 & 7.65 \\
\hline & & 15.3 & 2.90 & 20.3 & 8.92 & 25.3 & 7.61 \\
\hline & & 15.4 & 3.19 & 20.4 & 8.90 & 25.4 & 7.55 \\
\hline & & 15.5 & 3.47 & 20.5 & 8.88 & 25.5 & 7.50 \\
\hline & & 15.6 & 3.72 & 20.6 & 8.87 & 25.6 & 7.47 \\
\hline & & 15.7 & 4.02 & 20.7 & 8.84 & 25.7 & 7.42 \\
\hline & & 15.8 & 4.35 & 20.8 & 8.85 & 25.8 & 7.39 \\
\hline & & 15.9 & 4.69 & 20.9 & 8.89 & 25.9 & 7.36 \\
\hline & & 16.0 & 5.02 & 21.0 & 8.90 & 26.0 & 7.32 \\
\hline & & 16.1 & 5.31 & 21.1 & 8.92 & 26.1 & 7.28 \\
\hline & & 16.2 & 5.55 & 21.2 & 8.90 & 26.2 & 7.25 \\
\hline & & 16.3 & 5.73 & 21.3 & 8.88 & 26.3 & 7.23 \\
\hline & & 16.4 & 5.92 & 21.4 & 8.86 & 26.4 & 7.21 \\
\hline & & 16.5 & 6.10 & 21.5 & 8.87 & 26.5 & 7.20 \\
\hline & & 16.6 & 6.27 & 21.6 & 8.86 & 26.6 & 7.17 \\
\hline & & 16.7 & 6.48 & 21.7 & 8.87 & 26.7 & 7.14 \\
\hline & & 16.8 & 6.72 & 21.8 & 8.90 & 26.8 & 7.12 \\
\hline & & 16.9 & 6.97 & 21.9 & 8.89 & 26.9 & 7.10 \\
\hline & & 17.0 & 7.13 & 22.0 & 8.89 & 27.0 & 7.08 \\
\hline & & 17.1 & 7.27 & 22.1 & 8.89 & 27.1 & 7.06 \\
\hline & & 17.2 & 7.45 & 22.2 & 8.88 & 27.2 & 7.04 \\
\hline & & 17.3 & 7.67 & 22.3 & 8.87 & 27.3 & 7.05 \\
\hline & & 17.4 & 7.89 & 22.4 & 8.84 & 27.4 & 7.06 \\
\hline & & 17.5 & 8.04 & 22.5 & 8.83 & 27.5 & 7.07 \\
\hline & & 17.6 & 8.17 & 22.6 & 8.84 & 27.6 & 7.08 \\
\hline & & 17.7 & 8.27 & 22.7 & 8.82 & 27.7 & 7.09 \\
\hline & & 17.8 & 8.33 & 22.8 & 8.82 & 27.8 & 7.10 \\
\hline & & 17.9 & 8.41 & 22.9 & 8.81 & 27.9 & 7.11 \\
\hline 13.0 & -12.01 & 18.0 & 8.50 & 23.0 & 8.81 & 28.0 & 7.12 \\
\hline 13.1 & -10.63 & 18.1 & 8.59 & 23.1 & 8.80 & 28.1 & 7.13 \\
\hline 13.2 & -9.27 & 18.2 & 8.65 & 23.2 & 8.75 & 28.2 & 7.14 \\
\hline 13.3 & -8.13 & 18.3 & 8.69 & 23.3 & 8.69 & 28.3 & 7.14 \\
\hline 13.4 & -7.07 & 18.4 & 8.76 & 23.4 & 8.62 & 28.4 & 7.17 \\
\hline 13.5 & -6.11 & 18.5 & 8.84 & 23.5 & 8.57 & 28.5 & 7.20 \\
\hline 13.6 & -5.29 & 18.6 & 8.86 & 23.6 & 8.55 & 28.6 & 7.23 \\
\hline 13.7 & -4.45 & 18.7 & 8.87 & 23.7 & 8.50 & 28.7 & 7.25 \\
\hline 13.8 & -3.64 & 18.8 & 8.87 & 23.8 & 8.46 & 28.8 & 7.28 \\
\hline 13.9 & -2.88 & 18.9 & 8.90 & 23.9 & 8.41 & 28.9 & 7.30 \\
\hline 14.0 & -2.20 & 19.0 & 8.90 & 24.0 & 8.36 & 29.0 & 7.32 \\
\hline 14.1 & -1.61 & 19.1 & 8.92 & 24.1 & 8.31 & 29.1 & 7.34 \\
\hline 14.2 & -1.14 & 19.2 & 8.94 & 24.2 & 8.24 & 29.2 & 7.38 \\
\hline 14.3 & -.68 & 19.3 & 8.96 & 24.3 & 8.17 & 29.3 & 7.42 \\
\hline 14.4 & -.23 & 19.4 & 8.87 & 24.4 & 8.11 & 29.4 & 7.46 \\
\hline 14.5 & .17 & 19.5 & 8.95 & 24.5 & 8.04 & 29.5 & 7.50 \\
\hline 14.6 & .51 & 19.6 & 8.96 & 24.6 & 7.99 & 29.6 & 7.54 \\
\hline 14.7 & .88 & 19.7 & 8.99 & 24.7 & 7.95 & 29.7 & 7.57 \\
\hline 14.8 & 1.28 & 19.8 & 8.98 & 24.8 & 7.89 & 29.8 & 7.61 \\
\hline 14.9 & 1.68 & 19.9 & 8.96 & 24.9 & 7.83 & 29.9 & 7.65 \\
\hline 15.0 & 2.04 & 20.0 & 8.94 & 25.0 & 7.76 & 30.0 & 7.69 \\
\hline
\end{tabular}


The Corrections to Values of Temperature on the NBS-55 Scale. (The correction $\left(T_{68}-T_{\mathrm{NBS}-55}\right)$ is given with the argument in $\boldsymbol{T}_{\mathrm{NBS}-{ }_{55} \text { ) }}$-Continued

\begin{tabular}{|c|c|c|c|c|c|c|c|}
\hline $\begin{array}{l}T \\
\mathbf{K}\end{array}$ & $\begin{array}{c}\text { Corr. } \\
\text { mK }\end{array}$ & $\begin{array}{l}T \\
\mathbf{K}\end{array}$ & $\begin{array}{c}\text { Corr. } \\
\mathrm{mK}\end{array}$ & $\begin{array}{l}T \\
\mathbf{K}\end{array}$ & $\begin{array}{c}\text { Corr. } \\
\mathbf{m K}\end{array}$ & $\begin{array}{l}T \\
\mathrm{~K}\end{array}$ & $\begin{array}{c}\text { Corr. } \\
\mathrm{mK}\end{array}$ \\
\hline 30.0 & 7.69 & 35.0 & 11.03 & 40.0 & 15.42 & 45.0 & 15.12 \\
\hline 30.1 & 7.73 & 35.1 & 11.14 & 40.1 & 15.46 & 45.1 & 15.09 \\
\hline 30.2 & 7.75 & 35.2 & 11.26 & 40.2 & 15.50 & 45.2 & 15.06 \\
\hline 30.3 & 7.77 & 35.3 & 11.39 & 40.3 & 15.53 & 45.3 & 15.02 \\
\hline 30.4 & 7.79 & 35.4 & 11.51 & 40.4 & 15.57 & 45.4 & 14.99 \\
\hline 30.5 & 7.82 & 35.5 & 11.63 & 40.5 & 15.59 & 45.5 & 14.96 \\
\hline 30.6 & 7.85 & 35.6 & 11.75 & 40.6 & 15.62 & 45.6 & 14.92 \\
\hline 30.7 & 7.89 & 35.7 & 11.87 & 40.7 & 15.64 & 45.7 & 14.89 \\
\hline 30.8 & 7.92 & 35.8 & 11.98 & 40.8 & 15.66 & 45.8 & 14.85 \\
\hline 30.9 & 7.97 & 35.9 & 12.10 & 40.9 & 15.69 & 45.9 & 14.82 \\
\hline 31.0 & 8.00 & 36.0 & 12.22 & 41.0 & 15.71 & 46.0 & 14.80 \\
\hline 31.1 & 8.03 & 36.1 & 12.34 & 41.1 & 15.74 & 46.1 & 14.77 \\
\hline 31.2 & 8.06 & 36.2 & 12.46 & 41.2 & 15.76 & 46.2 & 14.74 \\
\hline 31.3 & 8.09 & 36.3 & 12.58 & 41.3 & 15.78 & 46.3 & 14.71 \\
\hline 31.4 & 8.13 & 36.4 & 12.69 & 41.4 & 15.79 & 46.4 & 14.69 \\
\hline 31.5 & 8.18 & 36.5 & 12.81 & 41.5 & 15.80 & 46.5 & 14.65 \\
\hline 31.6 & 8.22 & 36.6 & 12.91 & 41.6 & 15.80 & 46.6 & 14.62 \\
\hline 31.7 & 8.27 & 36.7 & 13.02 & 41.7 & 15.80 & 46.7 & 14.59 \\
\hline 31.8 & 8.31 & 36.8 & 13.12 & 41.8 & 15.81 & 46.8 & 14.56 \\
\hline 31.9 & 8.37 & 36.9 & 13.23 & 41.9 & 15.81 & 46.9 & 14.54 \\
\hline 32.0 & 8.42 & 37.0 & 13.32 & 42.0 & 15.81 & 47.0 & 14.50 \\
\hline 32.1 & 8.46 & 37.1 & 13.42 & 42.1 & 15.80 & 47.1 & 14.48 \\
\hline 32.2 & 8.53 & 37.2 & 13.52 & 42.2 & 15.80 & 47.2 & 14.44 \\
\hline 32.3 & 8.59 & 37.3 & 13.62 & 42.3 & 15.79 & 47.3 & 14.42 \\
\hline 32.4 & 8.64 & 37.4 & 13.71 & 42.4 & 15.79 & 47.4 & 14.39 \\
\hline 32.5 & 8.70 & 37.5 & 13.80 & 42.5 & 15.79 & 47.5 & 14.35 \\
\hline 32.6 & 8.76 & 37.6 & 13.89 & 42.6 & 15.77 & 47.6 & 14.32 \\
\hline 32.7 & 8.82 & 37.7 & 13.98 & 42.7 & 15.76 & 47.7 & 14.28 \\
\hline 32.8 & 8.88 & 37.8 & 14.06 & 42.8 & 15.74 & 47.8 & 14.25 \\
\hline 32.9 & 8.95 & 37.9 & 14.15 & 42.9 & 15.73 & 47.9 & 14.21 \\
\hline 33.0 & 9.02 & 38.0 & 14.24 & 43.0 & 15.71 & 48.0 & 14.17 \\
\hline 33.1 & 9.10 & 38.1 & 14.32 & 43.1 & 15.68 & 48.1 & 14.13 \\
\hline 33.2 & 9.19 & 38.2 & 14.39 & 43.2 & 15.67 & 48.2 & 14.08 \\
\hline 33.3 & 9.27 & 38.3 & 14.47 & 43.3 & 15.65 & 48.3 & 14.03 \\
\hline 33.4 & 9.35 & 38.4 & 14.54 & 43.4 & 15.63 & 48.4 & 13.98 \\
\hline 33.5 & 9.45 & 38.5 & 14.60 & 43.5 & 15.59 & 48.5 & 13.93 \\
\hline 33.6 & 9.54 & 38.6 & 14.66 & 43.6 & 15.56 & 48.6 & 13.88 \\
\hline 33.7 & 9.63 & 38.7 & 14.73 & 43.7 & 15.53 & 48.7 & 13.83 \\
\hline 33.8 & 9.73 & 38.8 & 14.80 & 43.8 & 15.51 & 48.8 & 13.78 \\
\hline 33.9 & 9.83 & 38.9 & 14.86 & 43.9 & 15.48 & 48.9 & 13.74 \\
\hline 33.0 & 9.92 & 39.0 & 14.92 & 44.0 & 15.45 & 49.0 & 13.68 \\
\hline 34.1 & 10.02 & 39.1 & 14.97 & 44.1 & 15.42 & 49.1 & 13.62 \\
\hline 34.2 & 10.13 & 39.2 & 15.02 & 44.2 & 15.39 & 49.2 & 13.56 \\
\hline 34.3 & 10.23 & 39.3 & 15.08 & 44.3 & 15.36 & 49.3 & 13.50 \\
\hline 34.4 & 10.34 & 39.4 & 15.14 & 44.4 & 15.33 & 49.4 & 13.45 \\
\hline 34.5 & 10.45 & 39.5 & 15.19 & 44.5 & 15.30 & 49.5 & 13.39 \\
\hline 34.6 & 10.57 & 39.6 & 15.24 & 44.6 & 15.27 & 49.6 & 13.33 \\
\hline 34.7 & 10.68 & 39.7 & 15.29 & 44.7 & 15.23 & 49.7 & 13.27 \\
\hline 34.8 & 10.81 & 39.8 & 15.34 & 44.8 & 15.19 & 49.8 & 13.21 \\
\hline 34.9 & 10.92 & 39.9 & 15.38 & 44.9 & 15.16 & 49.9 & 13.16 \\
\hline 35.0 & 11.03 & 40.0 & 15.42 & 45.0 & 15.12 & 50.0 & 13.09 \\
\hline
\end{tabular}


The Corrections to Values of Temperature on the NBS-55 Scale. (The correction $\left(T_{68}-T_{\text {NBS- 55 }}\right)$ is given with the argument in $\boldsymbol{T}_{\mathrm{NBS}-55^{\circ}}$ )-Continued

\begin{tabular}{|c|c|c|c|c|c|c|c|}
\hline $\begin{array}{l}T \\
\mathrm{~K}\end{array}$ & $\begin{array}{c}\text { Corr. } \\
\text { mK }\end{array}$ & $\begin{array}{l}T \\
\mathbf{K}\end{array}$ & $\begin{array}{c}\text { Corr. } \\
\mathbf{m K}\end{array}$ & $\begin{array}{l}T \\
\mathbf{K}\end{array}$ & $\begin{array}{c}\text { Corr. } \\
\text { mK }\end{array}$ & $\begin{array}{l}T \\
\mathbf{K}\end{array}$ & $\begin{array}{c}\text { Corr. } \\
\text { mK }\end{array}$ \\
\hline 50.0 & 13.09 & 55.0 & 7.07 & 60.0 & -.80 & 65.0 & -.09 \\
\hline 50.1 & 13.02 & 55.1 & 6.88 & 60.1 & -.88 & 65.1 & -.04 \\
\hline 50.2 & 12.96 & 55.2 & 6.70 & 60.2 & -.95 & 65.2 & .03 \\
\hline 50.3 & 12.90 & 55.3 & 6.51 & 60.3 & -1.03 & 65.3 & 09 \\
\hline 50.4 & 12.83 & 55.4 & 6.33 & 60.4 & -1.10 & 65.4 & 14 \\
\hline 50.5 & 12.77 & 55.5 & 6.13 & 60.5 & -1.16 & 65.5 & 20 \\
\hline 50.6 & 12.70 & 55.6 & 5.95 & 60.6 & -1.22 & 65.6 & .26 \\
\hline 50.7 & 12.63 & 55.7 & 5.76 & 60.7 & -1.26 & 65.7 & 32 \\
\hline 50.8 & 12.56 & 55.8 & 5.57 & 60.8 & -1.31 & 65.8 & .38 \\
\hline 50.9 & 12.48 & 55.9 & 5.38 & 60.9 & -1.36 & 65.9 & .44 \\
\hline 51.0 & 12.41 & 56.0 & 5.20 & 61.0 & -1.39 & 66.0 & .49 \\
\hline 51.1 & 12.33 & 56.1 & 5.02 & 61.1 & -1.42 & 66.1 & .54 \\
\hline 51.2 & 12.25 & 56.2 & 4.83 & 61.2 & -1.45 & 66.2 & .58 \\
\hline 51.3 & 12.18 & 56.3 & 4.65 & 61.3 & -1.47 & 66.3 & .63 \\
\hline 51.4 & 12.09 & 56.4 & 4.46 & 61.4 & -1.49 & 66.4 & .67 \\
\hline 51.5 & 12.01 & 56.5 & 4.28 & 61.5 & -1.50 & 66.5 & .72 \\
\hline 51.6 & 11.93 & 56.6 & 4.10 & 61.6 & -1.51 & 66.6 & .76 \\
\hline 51.7 & 11.84 & 56.7 & 3.92 & 61.7 & -1.51 & 66.7 & .80 \\
\hline 51.8 & 11.74 & 56.8 & 3.74 & 61.8 & -1.51 & 66.8 & 84 \\
\hline 51.9 & 11.65 & 56.9 & 3.56 & 61.9 & -1.51 & 66.9 & 87 \\
\hline 52.0 & 11.55 & 57.0 & 3.38 & 62.0 & -1.50 & 67.0 & 91 \\
\hline 52.1 & 11.45 & 57.1 & 3.21 & 62.1 & -1.48 & 67.1 & 94 \\
\hline 52.2 & 11.35 & 57.2 & 3.03 & 62.2 & -1.47 & 67.2 & 98 \\
\hline 52.3 & 11.24 & 57.3 & 2.86 & 62.3 & -1.45 & 67.3 & 1.01 \\
\hline 52.4 & 11.13 & 57.4 & 2.70 & 62.4 & -1.43 & 67.4 & 1.03 \\
\hline 52.5 & 11.02 & 57.5 & 2.53 & 62.2 & -1.40 & 67.5 & 1.05 \\
\hline 52.6 & 10.90 & 57.6 & 2.36 & 62.6 & -1.37 & 67.6 & 1.07 \\
\hline 52.7 & 10.77 & 57.7 & 2.19 & 62.7 & -1.34 & 67.7 & 1.08 \\
\hline 52.8 & 10.65 & 57.8 & 2.02 & 62.8 & -1.30 & 67.8 & 1.08 \\
\hline 52.9 & 10.52 & 57.9 & 1.86 & 62.9 & -1.26 & 67.9 & 1.09 \\
\hline 53.0 & 10.38 & 58.0 & 1.70 & 63.0 & -1.22 & 68.0 & 1.08 \\
\hline 53.1 & 10.24 & 58.1 & 1.55 & 63.1 & -1.17 & 68.1 & 1.08 \\
\hline 53.2 & 10.11 & 58.2 & 1.39 & 63.2 & -1.13 & 68.2 & 1.07 \\
\hline 53.3 & 9.97 & 58.3 & 1.24 & 63.3 & -1.08 & 68.3 & 1.06 \\
\hline 53.4 & 9.82 & 58.4 & 1.10 & 63.4 & -1.03 & 68.4 & 1.05 \\
\hline 53.5 & 9.66 & 58.5 & .95 & 63.5 & -.98 & 68.5 & 1.03 \\
\hline 53.6 & 9.51 & 58.6 & .81 & 63.6 & -.93 & 68.6 & 1.01 \\
\hline 53.7 & 9.35 & 58.7 & .68 & 63.7 & -.88 & 68.7 & .99 \\
\hline 53.8 & 9.19 & 58.8 & .54 & 63.8 & -.82 & 68.8 & 96 \\
\hline 53.9 & 9.02 & 58.9 & .41 & 63.9 & -.76 & 68.9 & 93 \\
\hline 54.0 & 8.85 & 59.0 & .28 & 64.0 & -.70 & 69.0 & 89 \\
\hline 54.1 & 8.69 & 59.1 & .15 & 64.1 & -.64 & 69.1 & 85 \\
\hline 54.2 & 8.52 & 59.2 & .02 & 64.2 & -.58 & 69.2 & 80 \\
\hline 54.3 & 8.34 & 59.3 & -.10 & 64.3 & -.52 & 69.3 & 75 \\
\hline 54.4 & 8.16 & 59.4 & -.21 & 64.4 & -.45 & 69.4 & 71 \\
\hline 54.5 & 7.98 & 59.5 & -.31 & 64.5 & -.40 & 69.5 & 65 \\
\hline 54.6 & 7.80 & 59.6 & -.42 & 64.6 & -.33 & 69.6 & 58 \\
\hline 54.7 & 7.62 & 59.7 & -.52 & 64.7 & -.28 & 69.7 & 52 \\
\hline 54.8 & 7.43 & 59.8 & -.62 & 64.8 & -.22 & 69.8 & 45 \\
\hline 54.9 & 7.25 & 59.9 & -.71 & 64.9 & -15 & 69.9 & 38 \\
\hline 55.0 & 7.07 & 60.0 & -.80 & 65.0 & -.09 & 70.0 & .31 \\
\hline
\end{tabular}


The Corrections to Values of Temperature on the NBS-55 Scale. (The correction $\left(T_{\mathrm{Bg}}-T_{\mathrm{NBS}-55}\right)$ is given with the argument in $\boldsymbol{T}_{\mathrm{NBS}-55}$.)-Continued

\begin{tabular}{|c|c|c|c|c|c|c|c|}
\hline $\begin{array}{l}T \\
\mathbf{K} \\
\end{array}$ & $\begin{array}{c}\text { Corr. } \\
\mathbf{m K}\end{array}$ & $\begin{array}{l}T \\
\mathrm{~K}\end{array}$ & $\begin{array}{c}\text { Corr. } \\
\text { mK }\end{array}$ & $\begin{array}{l}T \\
\mathrm{~K} \\
\end{array}$ & $\begin{array}{c}\text { Corr. } \\
\mathbf{m K}\end{array}$ & $\begin{array}{l}T \\
\mathbf{K}\end{array}$ & $\begin{array}{c}\text { Corr. } \\
\text { mK }\end{array}$ \\
\hline 70.0 & .31 & 75.0 & -5.59 & 80.0 & -9.03 & 85.0 & -2.91 \\
\hline 70.1 & .23 & 75.1 & -5.71 & 80.1 & -9.01 & 85.1 & -2.69 \\
\hline 70.2 & .15 & 75.2 & -5.84 & 80.2 & -8.98 & 85.2 & -2.47 \\
\hline 70.3 & .06 & 75.3 & -5.96 & 80.3 & -8.95 & 85.3 & -2.24 \\
\hline 70.4 & -.02 & 75.4 & -6.08 & 80.4 & -8.91 & 85.4 & -2.00 \\
\hline 70.5 & -.10 & 75.5 & -6.20 & 80.5 & -8.88 & 85.5 & -1.76 \\
\hline 70.6 & -.20 & 75.6 & -6.31 & 80.6 & -8.82 & 85.6 & -1.52 \\
\hline 70.7 & -.29 & 75.7 & -6.43 & 80.7 & -8.77 & 85.7 & -1.27 \\
\hline 70.8 & -.39 & 75.8 & -6.55 & 80.8 & -8.71 & 85.8 & -1.02 \\
\hline 70.9 & -.49 & 75.9 & -6.67 & 80.9 & -8.65 & 85.9 & -.76 \\
\hline 71.0 & -.59 & 76.0 & -6.78 & 81.0 & -8.59 & 86.0 & -.50 \\
\hline 71.1 & -.70 & 76.1 & -6.89 & 81.1 & -8.52 & 86.1 & -.24 \\
\hline 71.2 & -.81 & 76.2 & -7.00 & 81.2 & -8.44 & 86.2 & .02 \\
\hline 71.3 & -.92 & 76.3 & -7.11 & 81.3 & -8.36 & 86.3 & .29 \\
\hline 71.4 & -1.02 & 76.4 & -7.21 & 81.4 & -8.28 & 86.4 & .56 \\
\hline 71.5 & -1.13 & 76.5 & -7.31 & 81.5 & -8.19 & 86.5 & .83 \\
\hline 71.6 & -1.25 & 76.6 & -7.42 & 81.6 & -8.10 & 86.6 & 1.11 \\
\hline 71.7 & -1.36 & 76.7 & -7.51 & 81.7 & -8.00 & 86.7 & 1.38 \\
\hline 71.8 & -1.48 & 76.8 & -7.61 & 81.8 & -7.90 & 86.8 & 1.65 \\
\hline 71.9 & -1.60 & 76.9 & -7.71 & 81.9 & -7.80 & 86.9 & 1.92 \\
\hline 72.0 & -1.72 & 77.0 & -7.80 & 82.0 & -7.70 & 87.0 & 2.20 \\
\hline 72.1 & -1.85 & 77.1 & -7.89 & 82.1 & -7.59 & 87.1 & 2.47 \\
\hline 72.2 & -1.96 & 77.2 & -7.98 & 82.2 & -7.48 & 87.2 & 2.75 \\
\hline 72.3 & -2.08 & 77.3 & -8.06 & 82.3 & -7.36 & 87.3 & 3.02 \\
\hline 72.4 & -2.20 & 77.4 & -8.14 & 82.4 & -7.24 & 87.4 & 3.30 \\
\hline 72.5 & -2.32 & 77.5 & -8.22 & 82.5 & -7.12 & 87.5 & 3.57 \\
\hline 72.6 & -2.44 & 77.6 & -8.30 & 82.6 & -6.99 & 87.6 & 3.84 \\
\hline 72.7 & -2.58 & 77.7 & -8.37 & 82.7 & -6.86 & 87.7 & 4.12 \\
\hline 72.8 & -2.71 & 77.8 & -8.44 & 82.8 & -6.73 & 87.8 & 4.39 \\
\hline 72.9 & -2.84 & 77.9 & -8.51 & 82.9 & -6.59 & 87.9 & 4.65 \\
\hline 73.0 & -2.97 & 78.0 & -8.57 & 83.0 & -6.46 & 88.0 & 4.92 \\
\hline 73.1 & -3.10 & 78.1 & -8.63 & 83.1 & -6.32 & 88.1 & 5.18 \\
\hline 73.2 & -3.23 & 78.2 & -8.69 & 83.2 & -6.17 & 88.2 & 5.45 \\
\hline 73.3 & -3.36 & 78.3 & -8.74 & 83.3 & -6.02 & 88.3 & 5.70 \\
\hline 73.4 & -3.50 & 78.4 & -8.78 & 83.4 & -5.88 & 88.4 & 5.96 \\
\hline 73.5 & -3.64 & 78.5 & -8.83 & 83.5 & -5.72 & 88.5 & 6.22 \\
\hline 73.6 & -3.78 & 78.6 & -8.87 & 83.6 & -5.56 & 88.6 & 6.47 \\
\hline 73.7 & -3.91 & 78.7 & -8.91 & 83.7 & -5.40 & 88.7 & 6.72 \\
\hline 73.8 & -4.04 & 78.8 & -8.94 & 83.8 & -5.24 & 88.8 & 6.96 \\
\hline 73.9 & -4.17 & 78.9 & -8.97 & 83.9 & -5.07 & 88.9 & 7.21 \\
\hline 74.0 & -4.30 & 79.0 & -9.00 & 84.0 & -4.90 & 89.0 & 7.45 \\
\hline 74.1 & -4.43 & 79.1 & -9.03 & 84.1 & -4.72 & 89.1 & 7.68 \\
\hline 74.2 & -4.56 & 79.2 & -9.05 & 84.2 & -4.54 & 89.2 & 7.92 \\
\hline 74.3 & -4.69 & 79.3 & -9.07 & 84.3 & -4.34 & 89.3 & 8.15 \\
\hline 74.4 & -4.82 & 79.4 & -9.08 & 84.4 & -4.16 & 89.4 & 8.37 \\
\hline 74.5 & -4.95 & 79.5 & -9.08 & 84.5 & -3.96 & 89.5 & 8.58 \\
\hline 74.6 & -5.08 & 79.6 & -9.09 & 84.6 & -3.76 & 89.6 & 8.79 \\
\hline 74.7 & -5.20 & 79.7 & -9.08 & 84.7 & -3.56 & 89.7 & 9.00 \\
\hline 74.4 & -5.34 & 79.8 & -9.07 & 84.8 & -3.35 & 89.8 & 9.20 \\
\hline 74.9 & -5.46 & 79.9 & -9.06 & 84.9 & -3.13 & 89.9 & 9.40 \\
\hline 75.0 & -5.59 & 80.0 & -9.03 & 85.0 & -2.91 & 90.0 & 9.59 \\
\hline
\end{tabular}


Estimated Corrections to Values of Temperature on the IPTS of 1948. (The correction $\left(t_{68}-t_{48}\right)$ is given with the argument in $t_{48}$.)

\begin{tabular}{|c|c|c|c|c|c|c|c|}
\hline${ }^{t} \mathrm{C}$ & $\begin{array}{c}\text { Corr. } \\
{ }^{\circ} \mathrm{C}\end{array}$ & $\stackrel{t}{{ }^{\circ} \mathrm{C}}$ & $\begin{array}{l}\text { Corr. } \\
{ }^{\circ} \mathrm{C}\end{array}$ & $\stackrel{t}{{ }^{t} \mathrm{C}}$ & ${ }^{\circ} \mathrm{Corr}$. & $\stackrel{t}{{ }^{\circ} \mathrm{C}}$ & ${ }^{\circ} \mathrm{Corr}$. \\
\hline & & -150 & -.012630 & $-1 \dot{0}$ & .022386 & -50 & 02861 \\
\hline & & -149 & -.012963 & -99 & .023166 & -49 & 028183 \\
\hline & & -148 & -.013216 & -98 & .023921 & -48 & 027734 \\
\hline & & -147 & -.013392 & -97 & .024650 & -47 & .02727 \\
\hline & & -146 & -.013491 & -96 & .025352 & -46 & .02679 \\
\hline & & -145 & -.013514 & -95 & .026027 & -45 & .026311 \\
\hline & & -144 & -.013464 & -94 & .026675 & -44 & .025814 \\
\hline & & -143 & -.013343 & -93 & .027295 & -43 & .02530 \\
\hline & & -142 & -.013152 & -92 & .027886 & -42 & .024785 \\
\hline & & -141 & -.012893 & -91 & .028449 & -41 & 024256 \\
\hline & & -140 & -.012571 & -90 & .028983 & -40 & 023717 \\
\hline & & -139 & -.012186 & -89 & .029488 & -39 & 023170 \\
\hline & & -138 & -.011741 & -88 & .029963 & -38 & 022613 \\
\hline & & -137 & -.011240 & -87 & .030410 & -37 & 022049 \\
\hline & & -136 & -.010685 & -86 & .030827 & -36 & 021478 \\
\hline & & -135 & -.010079 & -85 & .031215 & -35 & 020899 \\
\hline & & -134 & -.009425 & -84 & .031574 & -34 & 02031 \\
\hline-183 & .007936 & -133 & -.008725 & -83 & .031904 & -33 & .019722 \\
\hline-182 & .009822 & -132 & -.007984 & -82 & .032204 & -32 & .019125 \\
\hline-181 & .011224 & -131 & -.007203 & -81 & .032476 & -31 & .01852 \\
\hline-180 & 012195 & -130 & -.006386 & -80 & .032720 & -30 & .017917 \\
\hline-179 & 012785 & -129 & -.005536 & -79 & .032934 & -29 & .017306 \\
\hline-178 & 013040 & -128 & -.004656 & -78 & .033121 & -28 & .016691 \\
\hline-177 & 0.13003 & -127 & -.003748 & -77 & .033280 & -27 & 016073 \\
\hline-176 & 012712 & -126 & -.002815 & -76 & .033411 & -26 & 015453 \\
\hline-175 & 012202 & -125 & -.001860 & -75 & .033515 & -25 & .014829 \\
\hline-174 & 011507 & -124 & -.000887 & -74 & .033593 & -24 & .014205 \\
\hline-173 & 010656 & -123 & .000104 & -73 & .033644 & -23 & .013578 \\
\hline-172 & 009676 & -122 & .001108 & -72 & .033669 & -22 & .012951 \\
\hline-171 & 008592 & -121 & .002123 & $-7 \tilde{1}$ & .033668 & -21 & .012323 \\
\hline-170 & 007427 & -120 & 003148 & -70 & .033642 & -20 & .011696 \\
\hline-169 & 006200 & -119 & 004179 & -69 & .033591 & -19 & .011069 \\
\hline-168 & 004931 & -118 & 005214 & -68 & .033517 & -18 & .010443 \\
\hline-167 & 003637 & -117 & 006250 & -67 & .033418 & -17 & .009819 \\
\hline-166 & 002332 & -116 & 007287 & -66 & .033296 & -16 & .009196 \\
\hline-165 & 001029 & -115 & .008321 & -65 & .033152 & -15 & .008577 \\
\hline-164 & -.000258 & -114 & .009351 & -64 & .032985 & -14 & .007960 \\
\hline-163 & -.001520 & -113 & .010375 & -63 & .032797 & -13 & .007347 \\
\hline-162 & -.002747 & -112 & .011390 & -62 & .032587 & -12 & .006739 \\
\hline-161 & -.003930 & -111 & .012396 & -61 & .032357 & $\begin{array}{l}-12 \\
-11\end{array}$ & .006136 \\
\hline-160 & -.005063 & -110 & .013390 & -60 & .032106 & $\begin{array}{l}-11 \\
-10\end{array}$ & .005537 \\
\hline-159 & -.006138 & -109 & .014370 & -59 & .031837 & $\begin{array}{r}-10 \\
-9\end{array}$ & \\
\hline-158 & -.007152 & -108 & .015337 & -58 & 031548 & -8 & .004360 \\
\hline-157 & -.008099 & -107 & .016287 & -57 & 031240 & -0 & .00378 \\
\hline-156 & -.008975 & -106 & .017220 & -56 & .030915 & -6 & .003213 \\
\hline-155 & -.009779 & -105 & .018134 & -55 & .030573 & -5 & .002652 \\
\hline-154 & -.010506 & -104 & .019028 & -54 & .030213 & -4 & .002100 \\
\hline-153 & -.011156 & -103 & 019901 & -53 & .029838 & -3 & .00155 \\
\hline-152 & -.011727 & -102 & .020753 & -52 & .029446 & -2 & .001029 \\
\hline-151 & -.012218 & -101 & .021581 & -51 & .029040 & $-\tilde{1}$ & .000511 \\
\hline-150 & -.012630 & -100 & .022386 & -50 & .028618 & 0 & .000005 \\
\hline
\end{tabular}


Estimated Corrections to Values of Temperature on the IPTS of 1948. (The correction (t.68 $\left.-t_{48}\right)$ is given with the argument in $\left.t_{48}\right)$-Continued

\begin{tabular}{|c|c|c|c|c|c|c|c|}
\hline $\begin{array}{l}{ }^{t} \mathrm{C} \\
{ }^{\circ} \mathrm{C}\end{array}$ & $\begin{array}{l}\text { Corr. } \\
{ }^{\circ} \mathrm{C}\end{array}$ & ${ }^{t} \mathrm{C}$ & $\begin{array}{l}\text { Corr. } \\
{ }^{\circ} \mathrm{C}\end{array}$ & ${ }^{t} \mathrm{C}$ & ${ }_{{ }^{\circ} \mathrm{C}}^{\text {Corr. }}$ & ${ }^{t} \mathrm{C}$ & $\begin{array}{l}\text { Corr. } \\
{ }^{\circ} \mathrm{C}\end{array}$ \\
\hline 0 & +.000005 & 50 & -.010366 & 100 & .000000 & 150 & .020374 \\
\hline 1 & -.000487 & 51 & -.010321 & 101 & .000341 & 151 & .020823 \\
\hline 2 & -.000966 & 52 & -.010269 & 102 & .000686 & 152 & .021273 \\
\hline 3 & -.001432 & 53 & -.010209 & 103 & .001034 & 153 & .021723 \\
\hline 4 & -.001884 & 54 & -.010141 & 104 & .001386 & 154 & .022174 \\
\hline 5 & -.002324 & 55 & -.010065 & 105 & .001742 & 155 & .022625 \\
\hline 6 & -.002750 & 56 & -.009981 & 106 & .002101 & 156 & .023077 \\
\hline 7 & -.003164 & 57 & -.009890 & 107 & .002464 & 157 & .023528 \\
\hline 8 & -.003565 & 58 & -.009791 & 108 & .002830 & 158 & .023981 \\
\hline 9 & -.003953 & 59 & -.009685 & 109 & .003200 & 159 & .024433 \\
\hline 10 & -.004329 & 60 & -.009571 & 110 & .003572 & 160 & .024886 \\
\hline 11 & -.004692 & 61 & -.009450 & 111 & .003948 & 161 & .025338 \\
\hline 12 & -.005043 & 62 & -.009322 & 112 & .004327 & 162 & .025791 \\
\hline 13 & -.005382 & 63 & -.009187 & 113 & .004710 & 163 & .026244 \\
\hline 14 & -.005709 & 64 & -.009045 & 114 & .005095 & 164 & .026696 \\
\hline 15 & -.006024 & 65 & -.008896 & 115 & .005483 & 165 & .027149 \\
\hline 16 & -.006328 & 66 & -.008740 & 116 & .005873 & 166 & .027602 \\
\hline 17 & -.006619 & 67 & -.008577 & 117 & .006267 & 167 & .028054 \\
\hline 18 & -.006899 & 68 & -.008408 & 118 & .006663 & 168 & .028506 \\
\hline 19 & -.007167 & 69 & -.008232 & 119 & .007062 & 169 & .028958 \\
\hline 20 & -.007424 & 70 & -.008050 & 120 & .007463 & 170 & .029409 \\
\hline 21 & -.007670 & 71 & -.007861 & 121 & .007867 & 171 & .029860 \\
\hline 22 & -.007905 & 72 & -.007666 & 122 & .008274 & 172 & .030311 \\
\hline 23 & -.008128 & 73 & -.007465 & 123 & .008682 & 173 & .030761 \\
\hline 24 & -.008341 & 74 & -.007258 & 124 & .009093 & 174 & .031211 \\
\hline 25 & -.008543 & 75 & -.007044 & 125 & .009507 & 175 & .031660 \\
\hline 26 & -.008734 & 76 & -.006825 & 126 & .009922 & 176 & .032109 \\
\hline 27 & -.008914 & 77 & -.006600 & 127 & .010339 & 177 & .032557 \\
\hline 28 & -.009084 & 78 & -.006369 & 128 & .010759 & 178 & .033004 \\
\hline 29 & -.009243 & 79 & -.006133 & 129 & .011180 & 179 & .033451 \\
\hline 30 & -.009392 & 80 & -.005891 & 130 & .011604 & 180 & .033896 \\
\hline 31 & -.009531 & 81 & -.005643 & 131 & .012029 & 181 & .034341 \\
\hline 32 & -.009660 & 82 & -.005390 & 132 & .012456 & 182 & .034785 \\
\hline 33 & -.009779 & 83 & -.005132 & 133 & .012884 & 183 & .035228 \\
\hline 34 & -.009888 & 84 & -.004868 & 134 & .013315 & 184 & .035670 \\
\hline 35 & -.009987 & 85 & -.004599 & 135 & .013747 & 185 & .036112 \\
\hline 36 & -.010076 & 86 & -.004325 & 136 & .014180 & 186 & .036552 \\
\hline 37 & -.010156 & 87 & -.004046 & 137 & .014615 & 187 & .036991 \\
\hline 38 & -.010226 & 88 & -.003763 & 138 & .015051 & 188 & .037429 \\
\hline 39 & -.010287 & 89 & -.003474 & 139 & .015489 & 189 & .037866 \\
\hline 40 & -.010339 & 90 & -.003180 & 140 & .015928 & 190 & .038301 \\
\hline 41 & -.010381 & 91 & -.002882 & 141 & .016368 & 191 & .038736 \\
\hline 42 & -.010414 & 92 & -.002579 & 142 & .016809 & 192 & .039169 \\
\hline 43 & -.010439 & 93 & -.002272 & 143 & .017251 & 193 & .039601 \\
\hline 44 & -.010454 & 94 & -.001960 & 144 & .017695 & 194 & .040031 \\
\hline 45 & -.010461 & 95 & -.001644 & 145 & .018139 & 195 & .040460 \\
\hline 46 & -.010459 & 96 & -.001323 & 146 & .018584 & 196 & .040888 \\
\hline 47 & -.010448 & 97 & -.000999 & 147 & .019031 & 197 & 041314 \\
\hline 48 & -.010429 & 98 & -.000670 & 148 & .019478 & 198 & .041739 \\
\hline 49 & -.010401 & 99 & -.000337 & 149 & .019925 & 199 & .042162 \\
\hline 50 & -.010366 & 100 & .000000 & 150 & .020374 & 200 & .042584 \\
\hline
\end{tabular}


Estimated Corrections to Values of Temperature on the IPTS of 1948. (The correction $\left(t_{68}-t_{48}\right)$ is given with the argument in $t_{48 \cdot}$ ) - Continued

\begin{tabular}{|c|c|c|c|c|c|c|c|}
\hline${ }^{t} \mathrm{C}$ & $\begin{array}{c}\text { Corr. } \\
{ }^{\circ} \mathrm{C}\end{array}$ & ${ }^{t} \mathrm{C}$ & ${ }^{\circ} \mathrm{Corr}$. & ${ }^{t} \mathrm{C}$ & $\begin{array}{l}\text { Corr. } \\
{ }^{\circ} \mathrm{C}\end{array}$ & ${ }^{t} \mathrm{C}$ & $\begin{array}{c}\text { Corr. } \\
{ }^{\circ} \mathrm{C}\end{array}$ \\
\hline 200 & .042584 & 250 & .061015 & 300 & 072611 & 350 & .076876 \\
\hline 201 & .043004 & 251 & .061320 & 301 & 072765 & 351 & .076897 \\
\hline 202 & .043422 & 252 & .061621 & 302 & 072916 & 352 & .076915 \\
\hline 203 & .043839 & 253 & .061921 & 303 & 073065 & 353 & .076931 \\
\hline 204 & .044254 & 254 & .062217 & 304 & 073210 & 354 & .076945 \\
\hline 205 & .044668 & 255 & .062510 & 305 & 073353 & 355 & .076957 \\
\hline 206 & .045079 & 256 & .062801 & 306 & 073492 & 356 & .076967 \\
\hline 207 & .045489 & 257 & .063088 & 307 & 073629 & 357 & 076975 \\
\hline 208 & .045897 & 258 & .063373 & 308 & 073763 & 358 & 076981 \\
\hline 209 & .046303 & 259 & .063655 & 309 & 073893 & 359 & .076985 \\
\hline 210 & .046707 & 260 & .063933 & 310 & 074021 & 360 & .076987 \\
\hline 211 & .047109 & 261 & .064209 & 311 & 074146 & 361 & 076987 \\
\hline 212 & .047510 & 262 & .064482 & 312 & 074267 & 362 & .076985 \\
\hline 213 & .047908 & 263 & .064752 & 313 & 074386 & 363 & 076981 \\
\hline 214 & .048304 & 264 & .065019 & 314 & 074502 & 364 & 076976 \\
\hline 215 & 048698 & 265 & .065283 & 315 & 074615 & 365 & .076969 \\
\hline 216 & 049091 & 266 & .065544 & 316 & 074726 & 366 & .076960 \\
\hline 217 & .049481 & 267 & .065802 & 317 & 074833 & 367 & .076949 \\
\hline 218 & 049869 & 268 & 066057 & 318 & 074937 & 368 & .076937 \\
\hline 219 & .050255 & 269 & .066309 & 319 & 075039 & 369 & .076923 \\
\hline 220 & .050638 & 270 & .066558 & 320 & 075138 & 370 & .076907 \\
\hline 221 & .051020 & 271 & .066804 & 321 & 075234 & 371 & 076890 \\
\hline 222 & .051399 & 272 & .067047 & 322 & 075327 & 372 & .076871 \\
\hline 223 & .051776 & 273 & .067287 & 323 & 075418 & 373 & .076851 \\
\hline 224 & .052150 & 274 & .067524 & 324 & 075505 & 374 & .076830 \\
\hline 225 & .052523 & 275 & .067758 & 325 & 075590 & 375 & 076806 \\
\hline 226 & .052893 & 276 & .067989 & 326 & 075673 & 376 & .076782 \\
\hline 227 & .053260 & 277 & .068217 & 327 & .075752 & 377 & .076756 \\
\hline 228 & .053626 & 278 & .068441 & 328 & 075829 & 378 & .076729 \\
\hline 229 & .053988 & 279 & .068663 & 329 & 075903 & 379 & .076701 \\
\hline 230 & .054349 & 280 & .068882 & 330 & .075975 & 380 & .076671 \\
\hline 231 & .054707 & 281 & .069097 & 331 & 076043 & 381 & .076640 \\
\hline 232 & .055062 & 282 & .069309 & 332 & 076110 & 382 & .076608 \\
\hline 233 & .055415 & 283 & .069519 & 333 & .076173 & 383 & .076575 \\
\hline 234 & .055766 & 284 & .069725 & 334 & .076234 & 384 & .076541 \\
\hline 235 & .056114 & 285 & .069928 & 335 & .076293 & 385 & .076505 \\
\hline 236 & .056459 & 286 & .070129 & 336 & 076349 & 386 & .076469 \\
\hline 237 & .056802 & 287 & .070326 & 337 & 076402 & 387 & .076432 \\
\hline 238 & .057142 & 288 & .070520 & 338 & .076453 & 388 & .076394 \\
\hline 239 & .057480 & 289 & .070711 & 339 & .076501 & 389 & .076355 \\
\hline 240 & .057814 & 290 & .070899 & 340 & 076547 & 390 & .076315 \\
\hline 241 & .058147 & 291 & .071084 & 341 & 076591 & 391 & .076274 \\
\hline 242 & .058476 & 292 & .071266 & 342 & .076632 & 392 & .076233 \\
\hline 243 & .058803 & 293 & .071444 & 343 & .076670 & 393 & .076191 \\
\hline 244 & .059127 & 294 & .071620 & 344 & 076707 & 394 & .076148 \\
\hline 245 & .059449 & 295 & .071793 & 345 & 076741 & 395 & .076105 \\
\hline 246 & .059768 & 296 & .071962 & 346 & .076772 & 396 & .076061 \\
\hline 247 & .060084 & 297 & .072129 & 347 & .076802 & 397 & .076016 \\
\hline 248 & .060397 & 298 & .072292 & 348 & .076829 & 398 & .075971 \\
\hline 249 & .060707 & 299 & .072453 & 349 & .076854 & 399 & .075926 \\
\hline 250 & .061015 & 300 & .072611 & 350 & .076876 & 400 & .075880 \\
\hline
\end{tabular}


Estimated Corrections to Values of Temperature on the IPTS of 1948. (The correction $\left(t_{\infty 8}-t_{48}\right)$ is given with the argument in $t_{48}$.)-Continued

\begin{tabular}{|c|c|c|c|c|c|c|c|}
\hline${ }^{t} \mathrm{C}$ & $\underset{{ }^{\circ} \mathrm{C}}{\text { Corr. }}$ & ${ }^{t} \mathrm{C}$ & ${ }_{{ }^{\circ} \mathrm{C}}^{\text {Corr. }}$ & ${ }^{t} \mathrm{C}$ & ${ }_{{ }^{\circ} \mathrm{C} .}^{\text {Corr. }}$ & ${ }^{t} \mathrm{C}$ & $\begin{array}{c}\text { Corr. } \\
{ }^{\circ} \mathrm{C} .\end{array}$ \\
\hline 400 & .075880 & 450 & .074257 & 500 & .079211 & 550 & .100515 \\
\hline 401 & .075834 & 451 & .074264 & 501 & .079440 & 551 & .101189 \\
\hline 402 & .075788 & 452 & .074273 & 502 & .079675 & 552 & .101874 \\
\hline 403 & .075741 & 453 & .074284 & 503 & .079917 & 553 & .102570 \\
\hline 404 & .075694 & 454 & .074298 & 504 & 080166 & 554 & .103278 \\
\hline 405 & .075648 & 455 & .074316 & 505 & .080422 & 555 & .103998 \\
\hline 406 & .075600 & 456 & .074336 & 506 & .080684 & 556 & .104730 \\
\hline 407 & .075553 & 457 & .074359 & 507 & .080954 & 557 & .105474 \\
\hline 408 & .075506 & 458 & .074385 & 508 & .081231 & 558 & .106231 \\
\hline 409 & .075459 & 459 & .074414 & 509 & .081514 & 559 & .106999 \\
\hline 410 & .075412 & 460 & .074446 & 510 & .081805 & 560 & .107780 \\
\hline 411 & .075366 & 461 & .074482 & 511 & .082104 & 561 & .108573 \\
\hline 412 & .075319 & 462 & .074521 & 512 & .082410 & 562 & .109379 \\
\hline 413 & .075273 & 463 & .074563 & 513 & .082723 & 563 & .110198 \\
\hline 414 & .075227 & 464 & .074609 & 514 & .083044 & 564 & .111030 \\
\hline 415 & .075181 & 465 & .074658 & 515 & .083373 & 565 & .111874 \\
\hline 416 & .075136 & 466 & .074711 & 516 & .083710 & 566 & .112732 \\
\hline 417 & .075091 & 467 & .074767 & 517 & .084054 & 567 & .113603 \\
\hline 418 & .075047 & 468 & .074828 & 518 & .084407 & 568 & .114487 \\
\hline 419 & .075004 & 469 & .074892 & 519 & .084768 & 569 & .115385 \\
\hline 420 & .074961 & 470 & .074960 & 520 & .085137 & 570 & .116296 \\
\hline 421 & .074918 & 471 & .075032 & 521 & .085514 & 571 & .117220 \\
\hline 422 & .074877 & 472 & .075108 & 522 & .085899 & 572 & .118159 \\
\hline 423 & .074836 & 473 & .075188 & 523 & 086293 & 573 & .119111 \\
\hline 424 & .074796 & 474 & .075273 & 524 & .086696 & 574 & .120078 \\
\hline 425 & .074757 & 475 & .075361 & 525 & .087108 & 575 & .121058 \\
\hline 426 & .074719 & 476 & .075455 & 526 & .087528 & 576 & .122053 \\
\hline 427 & .074682 & 477 & .075552 & 527 & .087957 & 577 & .123062 \\
\hline 428 & .074647 & 478 & .075654 & 528 & .088395 & 578 & .124086 \\
\hline 429 & .074612 & 479 & .075761 & 529 & .088842 & 579 & .125125 \\
\hline 430 & .074578 & 480 & .075872 & 530 & .089299 & 580 & .126178 \\
\hline 431 & .074546 & 481 & .075988 & 531 & .089764 & 581 & .127245 \\
\hline 432 & .074515 & 482 & .076109 & 532 & .090239 & 582 & .128328 \\
\hline 433 & .074486 & 483 & .076235 & 533 & .090724 & 583 & .129426 \\
\hline 434 & .074458 & 484 & .076366 & 534 & .091218 & 584 & .130539 \\
\hline 435 & .074431 & 485 & .076502 & 535 & .091722 & 585 & .131668 \\
\hline 436 & .074406 & 486 & .076644 & 536 & .092236 & 586 & .132812 \\
\hline 437 & .074383 & 487 & 076790 & 537 & .092759 & 587 & .133972 \\
\hline 438 & .074361 & 488 & .076942 & 538 & .093293 & 588 & .135147 \\
\hline 439 & .074341 & 489 & .077099 & 539 & .093837 & 589 & .136338 \\
\hline 440 & .074323 & 490 & .077262 & 540 & .094391 & 590 & .137545 \\
\hline 441 & .074307 & 491 & .077430 & 541 & .094955 & 591 & I 38768 \\
\hline 442 & .074293 & 492 & .077604 & 542 & .095530 & 592 & 140008 \\
\hline 443 & .074281 & 493 & .077784 & 543 & .096115 & 593 & .141264 \\
\hline 444 & .074271 & 494 & .077969 & 544 & .096711 & 594 & .142536 \\
\hline 445 & .074263 & 495 & .078161 & 545 & .097317 & 595 & .143825 \\
\hline 446 & .074257 & 496 & .078359 & 546 & .097935 & 596 & 145130 \\
\hline 447 & .074254 & 497 & .078562 & 547 & .098563 & 597 & 146453 \\
\hline 448 & .074252 & 498 & .078772 & 548 & .099203 & 598 & 147792 \\
\hline 449 & .074254 & 499 & 078988 & 549 & .099853 & 599 & 149148 \\
\hline 450 & .074257 & 500 & .079211 & 550 & 100515 & 600 & .150522 \\
\hline
\end{tabular}



Estimated Corrections to Values of Temperature on the IPTS of 1948. (The correction $\left(t_{G 8}-t_{48}\right)$ is given with the argument in $t_{48}$.)-Continued

\begin{tabular}{|c|c|c|c|c|c|c|c|}
\hline${ }^{\circ} \mathrm{C}$ & ${ }^{\circ} \mathrm{Corr}$ & $\stackrel{t}{{ }^{\circ} \mathrm{C}}$ & Corr. & ${ }^{t} \mathrm{C}$ & Corr. & ${ }^{t} \mathrm{C}$ & ${ }^{\circ} \mathrm{Corr}$. \\
\hline 600 & .150522 & 608 & .162148 & 616 & .174953 & 624 & .189006 \\
\hline 601 & .151913 & 609 & .163683 & 617 & .176640 & 625 & .190853 \\
\hline 602 & .153322 & 610 & .165236 & 618 & 178346 & 626 & .192722 \\
\hline 603 & .154748 & 611 & 166808 & 619 & .180073 & 627 & .194611 \\
\hline 604 & .156192 & 612 & .168399 & 620 & .181819 & 628 & .196522 \\
\hline 605 & .257654 & 613 & .170009 & 621 & .183585 & 629 & .198453 \\
\hline 606 & .159134 & 614 & .171637 & 622 & .185372 & 630 & .200406 \\
\hline 607 & .160632 & 615 & .173285 & 623 & .187178 & 631 & .202381 \\
\hline
\end{tabular}




\section{APPENDIX D. Table of the Function}

$M\left(t^{\prime}\right)=0.045\left(\frac{t^{\prime}}{100{ }^{\circ} \mathrm{C}}\right)\left(\frac{t^{\prime}}{100{ }^{\circ} \mathrm{C}}-1\right)\left(\frac{t^{\prime}}{419.58{ }^{\circ} \mathrm{C}}-1\right)\left(\frac{t^{\prime}}{630.74{ }^{\circ} \mathrm{C}}-1\right){ }^{\circ} \mathrm{C}$

and its derivative* from $t^{\prime}=0{ }^{\circ} \mathrm{C}$ to $t^{\prime}=630{ }^{\circ} \mathrm{C}$

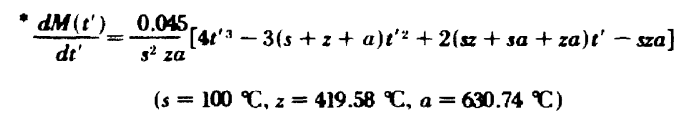

$\left(s=100^{\circ} \mathrm{C}, z=419.58{ }^{\circ} \mathrm{C}, a=630.74^{\circ} \mathrm{C}\right)$

\begin{tabular}{|c|c|c|c|c|c|}
\hline $\begin{array}{l}t^{\prime} \\
{ }^{\circ} \mathrm{C}\end{array}$ & $\begin{array}{c}M\left(t^{\prime}\right) \\
{ }^{\circ} \mathrm{C}\end{array}$ & $d M\left(t^{\prime}\right) / d t^{\prime}$ & $\begin{array}{l}t^{\prime} \\
{ }^{\circ} \mathrm{C} \\
\end{array}$ & $\begin{array}{c}M\left(t^{\prime}\right) \\
{ }^{\circ} \mathrm{C}\end{array}$ & $d M\left(t^{\prime}\right) / d t^{\prime}$ \\
\hline 0 & .000000 & -.000450 & 50 & -.009124 & .000040 \\
\hline 1 & -.000444 & -.000437 & 51 & -.009080 & .000048 \\
\hline 2 & -.000875 & -.000425 & 52 & -.009029 & .000055 \\
\hline 3 & -.001294 & -.000413 & 53 & -.008971 & .000062 \\
\hline 4 & -.001701 & -.000401 & 54 & -.008906 & .000068 \\
\hline 5 & -.002095 & -.000389 & 55 & -.008834 & .000075 \\
\hline 6 & -.002478 & -.000377 & 56 & -.008755 & .000082 \\
\hline 7 & -.002849 & -.000365 & 57 & -.008670 & .000089 \\
\hline 8 & -.003208 & -.000353 & 58 & -.008578 & .000095 \\
\hline 9 & -.003555 & -.000342 & 59 & -.008480 & .000101 \\
\hline 10 & -.003891 & -.000330 & 60 & -.008375 & .000108 \\
\hline 11 & -.004215 & -.000319 & 61 & -.008264 & .000114 \\
\hline 12 & -.004528 & -.000307 & 62 & -.008147 & .000120 \\
\hline 13 & -.004830 & -.000296 & 63 & -.008024 & .000126 \\
\hline 14 & -.005121 & -.000285 & 64 & -.007895 & .000132 \\
\hline 15 & -.005401 & -.000274 & 65 & -.007760 & .000138 \\
\hline 16 & -.005670 & -.000264 & 66 & -.007619 & .000144 \\
\hline 17 & -.005928 & -.000253 & 67 & -.007473 & .000149 \\
\hline 18 & -.006176 & -.000242 & 68 & -.007320 & .000155 \\
\hline 19 & -.006413 & -.000232 & 69 & -.007163 & .000160 \\
\hline 20 & -.006639 & -.000221 & 70 & -.007000 & .000166 \\
\hline 21 & -.006856 & -.000211 & 71 & -.006831 & .000171 \\
\hline 22 & -.007062 & -.000201 & 72 & -.006657 & .000176 \\
\hline 23 & -.007258 & -.000191 & 73 & -.006478 & .000182 \\
\hline 24 & -.007444 & -.000181 & 74 & -.006294 & .000187 \\
\hline 25 & -.007620 & -.000171 & 75 & -.006105 & .000192 \\
\hline 26 & -.007787 & -.000162 & 76 & -.005911 & .000196 \\
\hline 27 & -.007944 & -.000152 & 77 & -.005713 & .000201 \\
\hline 28 & $-.00809 i$ & -.000142 & 78 & -.005509 & .000206 \\
\hline 29 & -.008229 & -.000133 & 79 & -.005301 & .000210 \\
\hline 30 & -.008357 & -.000124 & 80 & -.005088 & .000215 \\
\hline 31 & -.008476 & -.000115 & 81 & -.004871 & .000219 \\
\hline 32 & -.008586 & -.000106 & 82 & -.004649 & .000224 \\
\hline 33 & -.008687 & -.000097 & 83 & -.004423 & .000228 \\
\hline 34 & -.008780 & -.000088 & 84 & -.004193 & .000232 \\
\hline 35 & -.008863 & -.000079 & 85 & -.003959 & .000236 \\
\hline 36 & -.008937 & -.000070 & 86 & -.003720 & .000240 \\
\hline 37 & -.009003 & -.000062 & 87 & -.003478 & .000244 \\
\hline 38 & -.009061 & -.000053 & 88 & -.003231 & .000248 \\
\hline 39 & -.009110 & -.000045 & 89 & -.002981 & .000252 \\
\hline 40 & -.009151 & -.000037 & 90 & -.002727 & .000256 \\
\hline 41 & -.009183 & -.000029 & 91 & -.002470 & .000259 \\
\hline 42 & -.009208 & -.000020 & 92 & -.002209 & .000263 \\
\hline 43 & -.009224 & -.000012 & 93 & -.001944 & .000266 \\
\hline 44 & -.009233 & -.000005 & 94 & -.001676 & .000270 \\
\hline 45 & -.009234 & .000003 & 95 & -.001404 & .000273 \\
\hline 46 & -.009227 & .000011 & 96 & -.001130 & .000276 \\
\hline 47 & -.009212 & .000018 & 97 & -.000852 & .000279 \\
\hline 48 & -.009190 & .000026 & 98 & -.000571 & .000283 \\
\hline 49 & -.009161 & .000033 & 99 & -.000287 & .000285 \\
\hline 50 & -.009124 & .000040 & 100 & .000000 & .000288 \\
\hline
\end{tabular}


Appendix D. Table of the Function-Continued

$$
M\left(t^{\prime}\right)=0.045\left(\frac{t^{\prime}}{100{ }^{\circ} \mathrm{C}}\right)\left(\frac{t^{\prime}}{100{ }^{\mathscr{C}}}-1\right)\left(\frac{t^{\prime}}{419.58{ }^{\circ} \mathrm{C}}-1\right)\left(\frac{t^{\prime}}{630.74{ }^{\circ} \mathrm{C}}-1\right){ }^{\circ} \mathrm{C}
$$

and its derivative* from $t^{\prime}=0^{\circ} \mathrm{C}$ to $t^{\prime}=630{ }^{\circ} \mathrm{C}$

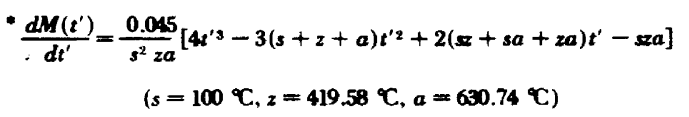

$\left(s=100^{\circ} \mathrm{C}, z=419.58{ }^{\circ} \mathrm{C}, a=630.74^{\circ} \mathrm{C}\right)$

\begin{tabular}{|c|c|c|c|c|c|}
\hline $\begin{array}{l}t^{\prime} \\
{ }^{\circ} \mathrm{C}\end{array}$ & $M_{{ }^{\circ} \mathrm{C}}\left(t^{\prime}\right)$ & $d M\left(t^{\prime}\right) / d t^{\prime}$ & $\begin{array}{l}t^{\prime} \\
{ }^{\circ} \mathrm{C}\end{array}$ & ${ }_{{ }^{\circ} \mathrm{C}}\left(t^{\prime}\right)$ & $d M\left(t^{\prime}\right) / d t^{\prime}$ \\
\hline 100 & -.000000 & .000288 & 150 & .016527 & .000345 \\
\hline 101 & .000290 & .000291 & 151 & .016872 & .000345 \\
\hline 102 & .000582 & .000294 & 152 & .017217 & .000344 \\
\hline 103 & .000878 & .000297 & 153 & .017560 & .000343 \\
\hline 104 & .001176 & .000299 & 154 & .017904 & .000343 \\
\hline 105 & .001476 & .000302 & 155 & .018246 & .000342 \\
\hline 106 & .001779 & .000304 & 156 & .018588 & .000341 \\
\hline 107 & .002085 & .000307 & 157 & .018929 & 000341 \\
\hline 108 & .002393 & .000309 & 158 & .019269 & .000340 \\
\hline 109 & .002703 & .000311 & 159 & .019608 & .000339 \\
\hline 110 & .003015 & .000313 & 160 & .019947 & .000338 \\
\hline 111 & .003330 & .000316 & 161 & .020284 & .000337 \\
\hline 112 & .003646 & .000318 & 162 & .020620 & .000336 \\
\hline 113 & .003965 & .000319 & 163 & .020956 & .000335 \\
\hline 114 & .004285 & .000321 & 164 & .021290 & .000334 \\
\hline 115 & .004608 & 000323 & 165 & .021623 & .000332 \\
\hline 116 & .004932 & .000325 & 166 & .021955 & .000331 \\
\hline 117 & .005257 & .000327 & 167 & .022285 & .000330 \\
\hline 118 & .005585 & .000328 & 168 & .022614 & .000328 \\
\hline 119 & .005914 & .000330 & 169 & .022942 & .000327 \\
\hline 120 & .006244 & .000331 & 170 & .023268 & .000326 \\
\hline 121 & .006576 & .000333 & 171 & .023593 & .000324 \\
\hline 122 & .006909 & .000334 & 172 & .023916 & .000322 \\
\hline 123 & .007244 & .000335 & 173 & .024238 & .000321 \\
\hline 124 & .007579 & .000336 & 174 & .024558 & .000319 \\
\hline 125 & .007916 & .000337 & 175 & .024876 & .000318 \\
\hline 126 & .008254 & .000339 & 176 & .025193 & .000316 \\
\hline 127 & .008593 & .000340 & 177 & .025508 & .000314 \\
\hline 128 & .008933 & .000340 & 178 & .025821 & .000312 \\
\hline 129 & .009274 & .000341 & 179 & .026132 & .000310 \\
\hline 130 & .009616 & .000342 & 180 & .026442 & .000308 \\
\hline 131 & .009958 & .000343 & 181 & .026749 & .000306 \\
\hline 132 & .010302 & .000343 & 182 & .027054 & .000304 \\
\hline 133 & .010645 & .000344 & 183 & .027358 & .000302 \\
\hline 134 & .010990 & .000345 & 184 & .027659 & .000300 \\
\hline 135 & .011335 & .000345 & 185 & .027958 & .000298 \\
\hline 136 & .011680 & .000346 & 186 & .028255 & .000296 \\
\hline 137 & .012026 & .000346 & 187 & .028550 & .000294 \\
\hline 138 & .012372 & .000346 & 188 & .028843 & .000291 \\
\hline 139 & .012718 & .000346 & 189 & .029133 & .000289 \\
\hline 140 & .013065 & .000347 & 190 & .029421 & .000287 \\
\hline 141 & .013411 & .000347 & 191 & .029707 & .000284 \\
\hline 142 & .013758 & .000347 & 192 & .029990 & .000282 \\
\hline 143 & .014105 & .000347 & 193 & .030271 & .000280 \\
\hline 144 & .014451 & .000347 & 194 & .030549 & .000277 \\
\hline 145 & .014798 & .000347 & 195 & .030825 & .000275 \\
\hline 146 & .015144 & .000346 & 196 & .031098 & .000272 \\
\hline 147 & .015491 & .000346 & 197 & .031369 & .000269 \\
\hline 148 & .015837 & .000346 & 198 & .031637 & .000267 \\
\hline 149 & .016182 & .000345 & 199 & .031902 & .000264 \\
\hline 150 & .016527 & .000345 & 200 & .032165 & .000261 \\
\hline
\end{tabular}


Appendix D. Table of the Function-Continued

$M\left(t^{\prime}\right)=0.045\left(\frac{t^{\prime}}{100{ }^{\circ} \mathrm{C}}\right)\left(\frac{t^{\prime}}{100{ }^{\circ} \mathrm{C}}-1\right)\left(\frac{t^{\prime}}{419.58{ }^{\circ} \mathrm{C}}-1\right)\left(\frac{t^{\prime}}{630.74{ }^{\circ} \mathrm{C}}-1\right){ }^{\circ} \mathrm{C}$

and its derivative* from $t^{\prime}=0{ }^{\circ} \mathrm{C}$ to $t^{\prime}=630{ }^{\circ} \mathrm{C}$

$\frac{d M\left(t^{\prime}\right)}{d t^{\prime}}=\frac{0.045}{s^{2} z a}\left[4 t^{\prime 3}-3(s+z+a) t^{\prime z}+2(s z+s a+z a) t^{\prime}-s z a\right]$
$\left(s=100^{\circ} \mathrm{C}, z=419.58^{\circ} \mathrm{C}, a=630.74^{\circ} \mathrm{C}\right)$

\begin{tabular}{|c|c|c|c|c|c|}
\hline $\begin{array}{l}t^{\prime} \\
{ }^{\circ} \mathrm{C}\end{array}$ & $\begin{array}{c}M\left(t^{\prime}\right) \\
\left.{ }^{\circ} \mathrm{C}\right)\end{array}$ & $d M\left(t^{\prime}\right) / d t^{\prime}$ & $\begin{array}{l}t^{\prime} \\
{ }^{\circ} \mathrm{C}\end{array}$ & $\begin{array}{c}M\left(t^{\prime}\right) \\
\left.{ }^{\circ} \mathrm{C}\right)\end{array}$ & $d M\left(t^{\prime}\right) / d t$ \\
\hline 200 & .032165 & 000261 & 250 & .041170 & 000088 \\
\hline 201 & .032425 & 000259 & 251 & .041256 & 000084 \\
\hline 202 & .032682 & 000256 & 252 & .041339 & .000080 \\
\hline 203 & .032937 & 000253 & 253 & .041417 & .000076 \\
\hline 204 & .033188 & 000250 & 254 & .041491 & .000072 \\
\hline 205 & .033437 & 000247 & 255 & .041561 & .000068 \\
\hline 206 & .033682 & .000244 & 256 & .041627 & 000064 \\
\hline 207 & .033925 & .000241 & 257 & .041689 & 000060 \\
\hline 208 & .034165 & .000238 & 258 & .041746 & .000056 \\
\hline 209 & .034402 & .000235 & 259 & .041800 & .000052 \\
\hline 210 & .034636 & .000232 & 260 & .041849 & .000047 \\
\hline 211 & .034866 & .000229 & 261 & .041895 & .000043 \\
\hline 212 & .035094 & .000226 & 262 & .041936 & .000039 \\
\hline 213 & .035318 & 000223 & 263 & .041973 & .000035 \\
\hline 214 & .035540 & .000220 & 264 & .042006 & .000031 \\
\hline 215 & .035758 & .000216 & 265 & .042034 & .000027 \\
\hline 216 & .035972 & .000213 & 266 & .042059 & .000022 \\
\hline 217 & .036184 & 000210 & 267 & .042079 & .000018 \\
\hline 218 & .036392 & 000207 & 268 & .042095 & .000014 \\
\hline 219 & .036597 & .000203 & 269 & .042107 & .000010 \\
\hline 220 & .036799 & 000200 & 270 & .042114 & .000005 \\
\hline 221 & .036997 & .000197 & 271 & .042117 & .000001 \\
\hline 222 & .037192 & 000193 & 272 & .042116 & -.000003 \\
\hline 223 & .037383 & .000190 & 273 & .042111 & -.000007 \\
\hline 224 & .037571 & 000186 & 274 & .042102 & -.000012 \\
\hline 225 & .037756 & 000183 & 275 & .042088 & -.000016 \\
\hline 226 & .037937 & .000179 & 276 & .042070 & -.000020 \\
\hline 227 & .038114 & .000176 & 277 & .042048 & -.000024 \\
\hline 228 & .038288 & .000172 & 278 & .042021 & -.000029 \\
\hline 229 & .038459 & .000169 & 279 & .041990 & -.000033 \\
\hline 230 & .038625 & 000165 & 280 & .041955 & -.000037 \\
\hline 231 & .038789 & 000161 & 281 & .041916 & -.000042 \\
\hline 232 & .038948 & .000158 & 282 & .041872 & -.000046 \\
\hline 233 & .039104 & .000154 & 283 & .041824 & -.000050 \\
\hline 234 & .039256 & .000150 & 284 & .041772 & -.000054 \\
\hline 235 & .039404 & .000147 & 285 & .041715 & -.000059 \\
\hline 236 & .039549 & .000143 & 286 & .041654 & -.000063 \\
\hline 237 & .039690 & .000139 & 287 & .041589 & -.000067 \\
\hline 238 & .039827 & .000135 & 288 & .041520 & -.000072 \\
\hline 239 & .039960 & .000131 & 289 & .041446 & -.000076 \\
\hline 240 & .040090 & .000128 & 290 & .041368 & -.000080 \\
\hline 241 & .040215 & .000124 & 291 & .041285 & -.000085 \\
\hline 242 & .040337 & .000120 & 292 & .041198 & -.000089 \\
\hline 243 & .040455 & .000116 & 293 & .041107 & -.000093 \\
\hline 244 & .040569 & .000112 & 294 & .041012 & -.000097 \\
\hline 245 & .040679 & .000108 & 295 & .040912 & -.000102 \\
\hline 246 & .040785 & .000104 & 296 & .040809 & -.000106 \\
\hline 247 & .040887 & .000100 & 297 & .040700 & -.000110 \\
\hline 248 & .040986 & .000096 & 298 & .040588 & -.000115 \\
\hline 249 & .041080 & .000092 & 299 & .040471 & -.000119 \\
\hline 250 & .041170 & .000088 & 300 & .040350 & -.000123 \\
\hline
\end{tabular}




\section{Appendix D. Table of the Function-Continued}

$$
M\left(t^{\prime}\right)=0.045\left(\frac{t^{\prime}}{100}\right)\left(\frac{t^{\prime}}{100}-1\right)\left(\frac{t^{\prime}}{419.58{ }^{\circ} \mathrm{C}}-1\right)\left(\frac{t^{\prime}}{630.74{ }^{\circ} \mathrm{C}}-1\right){ }^{\circ} \mathrm{C}
$$

and its derivative* from $t^{\prime}=0{ }^{\circ} \mathrm{C}$ to $t^{\prime}=630^{\circ} \mathrm{C}$

$$
\begin{gathered}
\frac{d M\left(t^{\prime}\right)}{d t^{\prime}}=\frac{0.045}{s^{2} z a}\left[4 t^{\prime 3}-3(s+z+a) t^{\prime 2}+2(s z+s a+z a) t^{\prime}-s z a\right] \\
\left(s=100^{\circ} \mathrm{C}, z=419.58^{\circ} \mathrm{C}, a=630.74^{\circ} \mathrm{C}\right)
\end{gathered}
$$

\begin{tabular}{|c|c|c|c|c|c|}
\hline $\begin{array}{l}t^{\prime} \\
{ }^{\circ} \mathrm{C}\end{array}$ & ${ }_{{ }^{\circ} \mathrm{C}}^{M\left(t^{\prime}\right)}$ & $d M\left(t^{\prime}\right) / d t^{\prime}$ & $\stackrel{\circ}{ }^{\prime} \mathrm{C}$ & $M_{{ }^{\circ} \mathrm{C}}\left(t^{\prime}\right)$ & $d M\left(t^{\prime}\right) / d t^{\prime}$ \\
\hline 300 & .040350 & -.000123 & 350 & .029063 & -.000322 \\
\hline 301 & .040225 & -.000127 & 351 & .028740 & -.000325 \\
\hline 302 & .040095 & -.000132 & 352 & .028412 & -.000329 \\
\hline 303 & .03996 & -.000136 & 353 & .028082 & -.000332 \\
\hline 304 & .039823 & -.000140 & 354 & .027748 & -.000336 \\
\hline 305 & .039681 & -.000144 & 355 & .027410 & -.000339 \\
\hline 306 & .039534 & -.000149 & 356 & .027069 & -.000343 \\
\hline 307 & .039383 & -.000153 & 357 & .026725 & -.000346 \\
\hline 308 & .039228 & -.000157 & 358 & .026378 & -.000349 \\
\hline 309 & .039069 & -.000161 & 359 & .026027 & -.000352 \\
\hline 310 & .038906 & -.000166 & 360 & .025673 & -.000356 \\
\hline 311 & .038738 & -.000170 & 361 & .025316 & -.000359 \\
\hline 312 & .038566 & -.000174 & 362 & .024955 & -.000362 \\
\hline 313 & .038390 & -.000178 & 363 & .024592 & -.000365 \\
\hline 314 & .038210 & -.000182 & 364 & .024225 & -.000368 \\
\hline 315 & .038025 & -.000186 & 365 & .023855 & -.000371 \\
\hline 316 & .037837 & -.000191 & 366 & .023482 & -.000375 \\
\hline 317 & .037644 & -.000195 & 367 & .023106 & -.000378 \\
\hline 318 & .037447 & -.000199 & 368 & .022727 & -.000381 \\
\hline 319 & .037247 & -.000203 & 369 & .022345 & -.000384 \\
\hline 320 & .037042 & -.000207 & 370 & .021960 & -.000386 \\
\hline 321 & .036832 & -.000211 & 371 & .021572 & -.000389 \\
\hline 322 & .036619 & -.000215 & 372 & .021181 & -.000392 \\
\hline 323 & .036402 & -.000219 & 373 & .020787 & -.000395 \\
\hline 324 & .036181 & -.000223 & 374 & .020391 & -.000398 \\
\hline 325 & .035955 & -.000227 & 375 & .019992 & -.000401 \\
\hline 326 & .035726 & -.000231 & 376 & .019590 & -.000403 \\
\hline 327 & .035493 & -.000235 & 377 & .019185 & -.000406 \\
\hline 328 & .035255 & -.000239 & 378 & .018778 & -.000409 \\
\hline 329 & .035014 & -.000243 & 379 & .018368 & -.000411 \\
\hline 330 & .034769 & -.000247 & 380 & .017955 & -.000414 \\
\hline 331 & .034520 & -.000251 & 381 & .017540 & -.000416 \\
\hline 332 & .034267 & -.000255 & 382 & .017122 & -.000419 \\
\hline 333 & .034010 & -.000259 & 383 & .016702 & -.000421 \\
\hline 334 & .033749 & -.000263 & 384 & .016280 & -.000424 \\
\hline 335 & .033484 & -.000267 & 385 & .015855 & -.000426 \\
\hline 336 & .033215 & -.000271 & 386 & .015427 & -.000429 \\
\hline 337 & .032943 & -.000274 & 387 & .014997 & -.000431 \\
\hline 338 & .032667 & -.000278 & 388 & .014565 & -.000433 \\
\hline 339 & .032387 & -.000282 & 389 & .014131 & -.000435 \\
\hline 340 & .032103 & -.000286 & 390 & .013695 & -.000438 \\
\hline 341 & .031816 & -.000289 & 391 & .013256 & -.000440 \\
\hline 342 & .031524 & -.000293 & 392 & .012815 & -.000442 \\
\hline 343 & .031229 & -.000297 & 393 & .012373 & -.000444 \\
\hline 344 & .030931 & -.000300 & 394 & .011928 & -.000446 \\
\hline 345 & .030629 & -.000304 & 395 & .011481 & -.000448 \\
\hline 346 & .030323 & -.000308 & 396 & .011032 & -.000450 \\
\hline 347 & .030013 & -.000311 & 397 & .010582 & -.000452 \\
\hline 348 & .029700 & -.000315 & 398 & .010129 & -.000453 \\
\hline 349 & .029383 & -.000318 & 399 & .009675 & -.000455 \\
\hline 350 & .029063 & -.000322 & 400 & .009219 & -.000457 \\
\hline
\end{tabular}


Appendix D. Table of the Function-Continued

$$
M\left(t^{\prime}\right)=0.045\left(\frac{t^{\prime}}{100 \mathscr{C}}\right)\left(\frac{t^{\prime}}{100{ }^{\circ}}-1\right)\left(\frac{t^{\prime}}{419.58{ }^{\circ} \mathrm{C}}-1\right)\left(\frac{t^{\prime}}{630.74{ }^{\circ} \mathrm{C}}-1\right){ }^{\circ} \mathrm{C}
$$

and its derivative* from $t^{\prime}=0{ }^{\circ} \mathrm{C}$ to $t^{\prime}=630{ }^{\circ} \mathrm{C}$

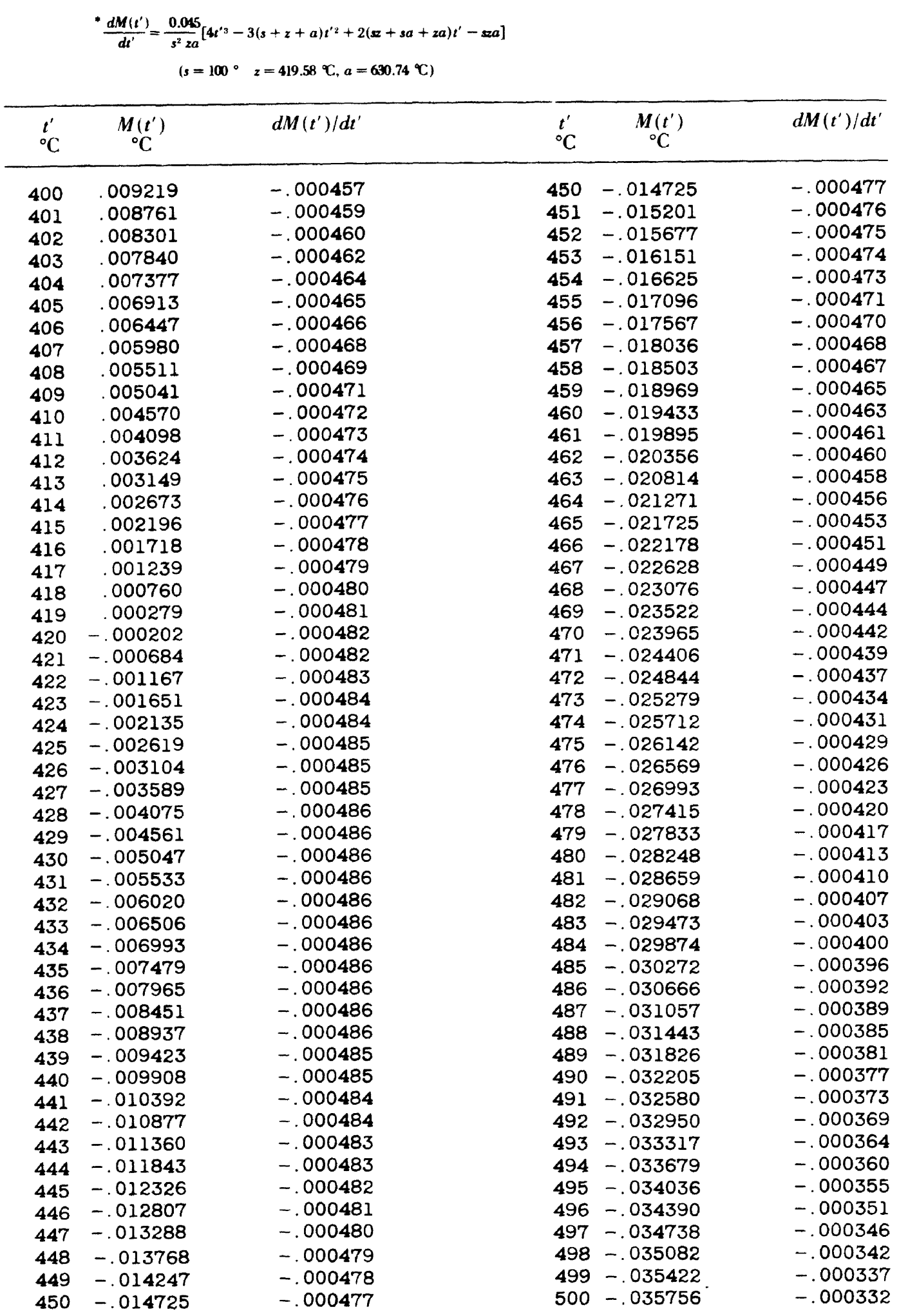


Appendix D. Table of the Function-Continued

$M\left(t^{\prime}\right)=0.045\left(\frac{t^{\prime}}{100{ }^{\circ} \mathrm{C}}\right)\left(\frac{t^{\prime}}{100{ }^{\circ} \mathrm{C}}-1\right)\left(\frac{t^{\prime}}{419.58{ }^{\circ} \mathrm{C}}-1\right)\left(\frac{t^{\prime}}{630.74{ }^{\circ} \mathrm{C}}-1\right){ }^{\circ} \mathrm{C}$

and its derivative* from $t^{\prime}=0^{\circ} \mathrm{C}$ to $t^{\prime}=630{ }^{\circ} \mathrm{C}$

$\frac{d M\left(t^{\prime}\right)}{d t^{\prime}}=\frac{0.045}{s^{2} z a}\left[4 t^{\prime 3}-3(s+z+a) t^{\prime 2}+2(s z+s a+z a) t^{\prime}-s z a\right]$
$\left(s=100^{\circ} \mathrm{C}, z=419.58^{\circ} \mathrm{C} . a^{2}=630.74^{\circ} \mathrm{C}\right)$

\begin{tabular}{|c|c|c|c|c|c|}
\hline $\begin{array}{l}t^{\prime} \\
{ }^{\circ} \mathrm{C}\end{array}$ & $\begin{array}{c}M\left(t^{\prime}\right) \\
\left.{ }^{\circ} \mathrm{C}\right)\end{array}$ & $d M\left(t^{\prime}\right) / d t^{\prime}$ & $\begin{array}{l}t^{\prime} \\
{ }^{o} \mathrm{C}\end{array}$ & $\begin{array}{c}M\left(t^{\prime}\right) \\
\left.{ }^{\circ} \mathrm{C}\right)\end{array}$ & $d M\left(t^{\prime}\right) / d t^{\prime}$ \\
\hline 500 & -.035756 & -.000332 & 550 & -.044316 & .000030 \\
\hline 501 & -.036086 & -.000327 & 551 & -.044281 & 000040 \\
\hline 502 & -.036410 & -.000322 & 552 & -.044236 & 000050 \\
\hline 503 & -.036730 & -.000317 & 553 & -.044181 & .000060 \\
\hline 504 & -.037044 & -.000312 & 554 & -.044116 & 000070 \\
\hline 505 & -.037353 & -.000306 & 555 & -.044041 & 000080 \\
\hline 506 & -.037657 & -.000301 & 556 & -.043956 & .000090 \\
\hline 507 & -.037955 & -.000296 & 557 & -.043860 & 000101 \\
\hline 508 & -.038248 & -.000290 & 558 & -.043754 & .000111 \\
\hline 509 & -.038535 & -.000284 & 559 & -.043637 & 000122 \\
\hline 510 & -.038817 & -.000279 & 560 & -.043510 & 000133 \\
\hline 511 & -.039092 & -.000273 & 561 & -.043371 & .000144 \\
\hline 512 & -.039362 & -.000267 & 562 & $\cdots .043222$ & .000155 \\
\hline 513 & -.039626 & -.000261 & 563 & -.043062 & .000166 \\
\hline 514 & -.039884 & -.000255 & 564 & -.042890 & .000177 \\
\hline 515 & -.040135 & -.000248 & 565 & -.042707 & .000189 \\
\hline 516 & -.040381 & -.000242 & 566 & -.042513 & .000200 \\
\hline 517 & -.040620 & -.000236 & 567 & -.042307 & .000212 \\
\hline 518 & -.040852 & -.000229 & 568 & -.042090 & .000223 \\
\hline 519 & -.041078 & -.000223 & 569 & -.041861 & .000235 \\
\hline 520 & -.041298 & -.000216 & 570 & -.041620 & .000247 \\
\hline 521 & -.041510 & -.000209 & 571 & -.041367 & .000259 \\
\hline 522 & -.041716 & -.000202 & 572 & -.041102 & .000271 \\
\hline 523 & -.041915 & -.000195 & 573 & -.040825 & 000283 \\
\hline 524 & -.042107 & -.000188 & 574 & -.040535 & .000296 \\
\hline 525 & -.042292 & -.000181 & 575 & -.040233 & .000308 \\
\hline 526 & -.042470 & -.000174 & 576 & -.039918 & .000321 \\
\hline 527 & -.042640 & -.000167 & 577 & -.039591 & .000334 \\
\hline 528 & -.042803 & -.000159 & 578 & -.039251 & .000346 \\
\hline 529 & -.042959 & -.000152 & 579 & -.038898 & .000359 \\
\hline 530 & -.043106 & -.000144 & 580 & -.038532 & .000373 \\
\hline 531 & -.043247 & -.000136 & 581 & -.038153 & .000386 \\
\hline 532 & -.043379 & -.000128 & 582 & -.037761 & .000399 \\
\hline 533 & -.043504 & -.000121 & 583 & -.037355 & .000412 \\
\hline 534 & -.043620 & -.000113 & 584 & -.036936 & .000426 \\
\hline 535 & -.043729 & -.000104 & 585 & -.036503 & .000440 \\
\hline 536 & -.043829 & -.000096 & 586 & -.036056 & 000454 \\
\hline 537 & -.043921 & -.000088 & 587 & -.035596 & 000467 \\
\hline 538 & -.044004 & -.000079 & 588 & -.035121 & 000482 \\
\hline 539 & -.044080 & -.000071 & 589 & -.034633 & 000496 \\
\hline 540 & -.044146 & -.000062 & 590 & -.034130 & 000510 \\
\hline 541 & -.044204 & -.000053 & 591 & -.033613 & .000524 \\
\hline 542 & -.044253 & -.000045 & 592 & -.033081 & .000539 \\
\hline 543 & -.044293 & -.000036 & 593 & -.032535 & .000554 \\
\hline 544 & -.044324 & -.000027 & 594 & -.031974 & .000568 \\
\hline 545 & -.044346 & -.000017 & 595 & -.031398 & 000583 \\
\hline 546 & -.044359 & -.000008 & 596 & -.030807 & 000598 \\
\hline 547 & -.044362 & .000001 & 597 & -.030201 & 000614 \\
\hline 548 & -.044356 & .000011 & 598 & -.029580 & 000629 \\
\hline 549 & -.044341 & .000020 & 599 & -.028944 & 000644 \\
\hline 550 & -.044316 & .000030 & 600 & -.028292 & .000660 \\
\hline
\end{tabular}


Appendix D. Table of the Function-Continued

$M\left(t^{\prime}\right)=0.045\left(\frac{t^{\prime}}{100{ }^{\circ} \mathrm{C}}\right)\left(\frac{t^{\prime}}{100{ }^{\circ} \mathrm{C}}-1\right)\left(\frac{t^{\prime}}{419.58{ }^{\circ} \mathrm{C}}-1\right)\left(\frac{t^{\prime}}{6^{\prime} 0^{\circ}-14}-1\right){ }^{\circ} \mathrm{C}$ and its derivative* from $t^{\prime}=0^{\circ} \mathrm{C}$ to $t^{\prime}=630{ }^{\circ} \mathrm{C}$

$$
\begin{gathered}
\frac{d M\left(t^{\prime}\right)}{d t^{\prime}}=\frac{0.045}{s^{2} z a}\left[4 t^{\prime 3}-3(s+z+a) t^{\prime z}+2(s z+s a+z a) t^{\prime}-s z a\right] \\
\left(s=100^{\circ} \mathrm{C}, z=419.58^{\circ} \mathrm{C}, a=630.74^{\circ} \mathrm{C}\right)
\end{gathered}
$$

\begin{tabular}{cccccc}
\hline $\begin{array}{c}t^{\prime} \\
{ }^{\circ} \mathrm{C}\end{array}$ & $\begin{array}{c}M\left(t^{\prime}\right) \\
{ }^{\circ} \mathrm{C}\end{array}$ & $d M\left(t^{\prime}\right) / d t^{\prime}$ & $\begin{array}{c}t^{\prime} \\
{ }^{\circ} \mathrm{C}\end{array}$ & $\begin{array}{c}M\left(t^{\prime}\right) \\
{ }^{\circ} \mathrm{C}\end{array}$ & $d M\left(t^{\prime}\right) / d t^{\prime}$ \\
\hline 600 & -.028292 & .000660 & $616-.015648$ & .000926 \\
601 & -.027624 & .000675 & 617 & -.014713 & .000944 \\
602 & -.026941 & .000691 & 618 & -.013760 & .000962 \\
603 & -.026241 & .000707 & 619 & -.012789 & .000980 \\
604 & -.025526 & .000723 & $620-.011800$ & .000998 \\
605 & -.024795 & .000739 & $621-.010793$ & .001016 \\
606 & -.024047 & .000756 & $622-.009767$ & .001035 \\
607 & -.023283 & .000772 & 623 & -.008723 & .001053 \\
608 & -.022503 & .000789 & 624 & -.007660 & .001072 \\
609 & -.021705 & .000806 & $625-.006579$ & .001091 \\
610 & -.020891 & .000822 & $626-.005478$ & .001110 \\
611 & -.020061 & .000839 & 627 & -.004359 & .001129 \\
612 & -.019213 & .000856 & 628 & -.003220 & .001148 \\
613 & -.018348 & .000874 & $629-.002062$ & .001168 \\
614 & -.017465 & .000891 & $630-.000884$ & .001187 \\
615 & -.016566 & .000909 & 631 & .000313 & .001207 \\
\hline
\end{tabular}




\section{APPENDIX E. Tables of Values of the Reference Function and Its Derivative from $T=13 \mathrm{~K}$ to $T=273.15 \mathrm{~K}$ and over the Equivalent Range of Temperature With the Argument in Degrees Celsius}

$W^{*}(T)$ and its derivative are defined by the expressions

$$
T=\sum_{i=0}^{20} A_{i}\left[\ln W^{*}(T)\right]^{i}
$$

and

$$
\frac{d W^{*}(T)}{d T}=\frac{1}{\frac{1}{W^{*}(T)} \sum_{i=1}^{20} i A_{i}\left[\ln W^{*}(T)\right]^{i-1}}
$$

where $T=T_{68}, \quad W^{*}(T)=W C C T_{-68}\left(T_{68}\right)$, and the

\begin{tabular}{|c|c|c|c|}
\hline$i$ & $A_{i}$ & $i$ & $A_{i}$ \\
\hline 0 & $0.27315 \times 10^{3}$ & 11 & $0.7679763581708458 \times 10$ \\
\hline 1 & $0.2508462096788033 \times 10^{3}$ & 12 & $0.2136894593828500 \times 10$ \\
\hline 2 & $0.1350998699649997 \times 10^{3}$ & 13 & 0.4598433489280693 \\
\hline 3 & $0.5278567590085172 \times 10^{2}$ & 14 & $0.7636146292316480 \times 10^{-1}$ \\
\hline 4 & $0.2767685488541052 \times 10^{2}$ & 15 & $0.9693286203731213 \times 10^{-2}$ \\
\hline 5 & $0.3910532053766837 \times 10^{2}$ & 16 & $0.9230691540070075 \times 10^{-3}$ \\
\hline 6 & $0.6556132305780693 \times 10^{2}$ & 17 & $0.6381165909526538 \times 10^{-4}$ \\
\hline 7 & $0.8080358685598667 \times 10^{2}$ & 18 & $0.3022932378746192 \times 10^{-5}$ \\
\hline 8 & $0.7052421182340520 \times 10^{2}$ & 19 & $0.8775513913037602 \times 10^{-7}$ \\
\hline 9 & $0.4478475896389657 \times 10^{2}$ & 20 & $0.1177026131254774 \times 10^{-8}$ \\
\hline 10 & $0.2125256535560578 \times 10^{2}$ & & \\
\hline
\end{tabular}
values of $A_{i}$ are: 
The function $W^{*}(T)$ and its derivative were evaluated and printed employing the following FORTRAN routine:

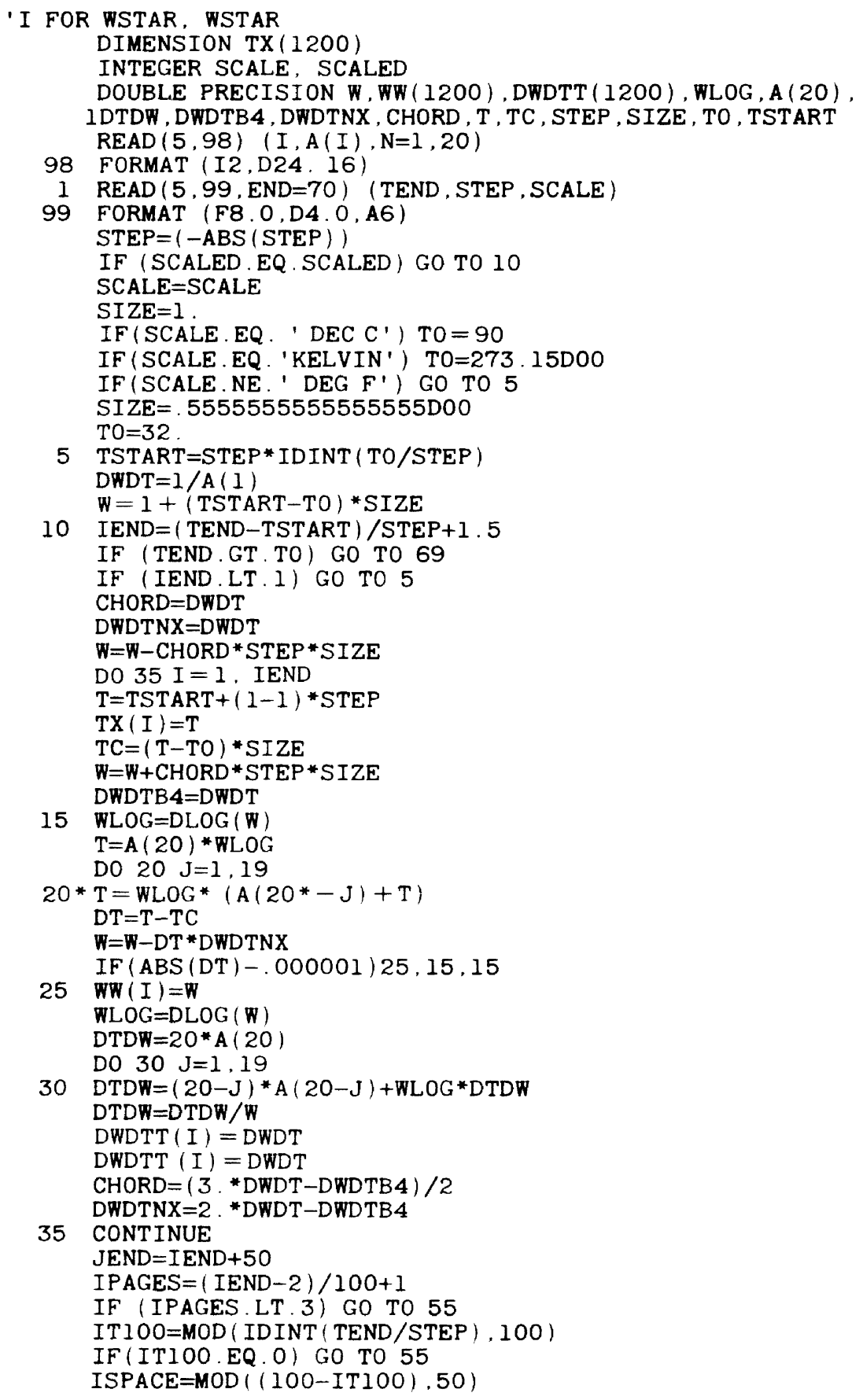




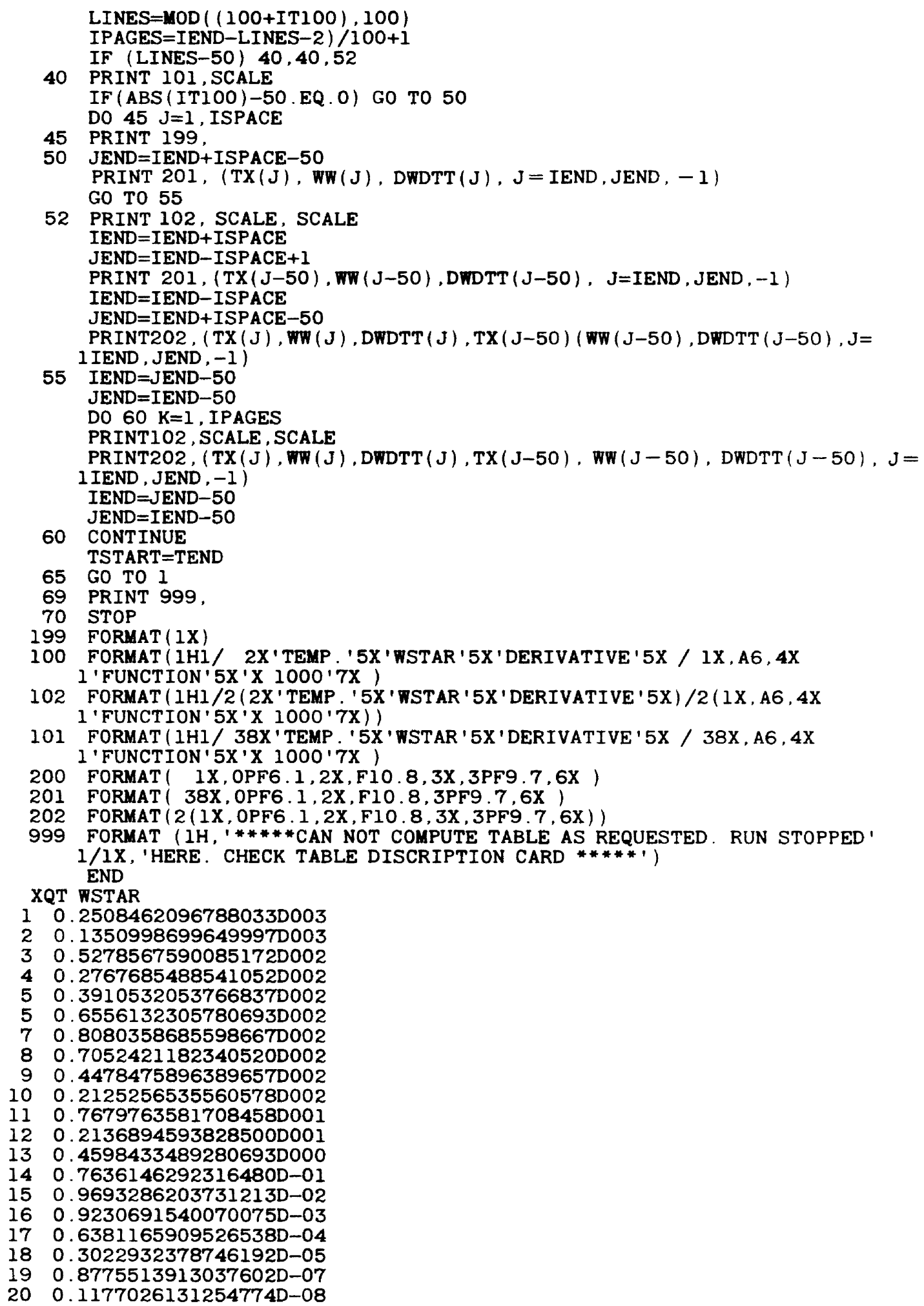


IPTS-68 Reference Function and Its First Derivative With the Argument in Kelvin

\begin{tabular}{|c|c|c|c|c|c|}
\hline $\begin{array}{l}T \\
\mathrm{~K}\end{array}$ & $W^{*}(T)$ & $\begin{array}{c}d W^{*}(T) / d T \\
\times 1000 \\
\mathrm{~K}^{-1}\end{array}$ & $\begin{array}{l}T \\
\mathrm{~K}\end{array}$ & $W^{*}(T)$ & $\begin{array}{c}d W^{*}(T) / d T \\
\quad \times 1000 \\
\mathrm{~K}^{-1}\end{array}$ \\
\hline & & & 15.0 & .00174542 & .3163198 \\
\hline & & & 15.1 & .00177737 & .3227158 \\
\hline & & & 15.2 & .00180996 & .3291831 \\
\hline & & & 15.3 & .00184321 & .3357224 \\
\hline & & & 15.4 & .00187711 & .3423344 \\
\hline & & & 15.5 & .00191168 & .3490197 \\
\hline & & & 15.6 & .00194692 & .3557792 \\
\hline & & & 15.7 & .00198283 & .3626135 \\
\hline & & & 15.8 & .00201944 & .3695234 \\
\hline & & & 15.9 & .00205674 & .3765094 \\
\hline & & & 16.0 & .00209474 & .3835722 \\
\hline & & & 16.1 & .00213346 & .3907123 \\
\hline & & & 16.2 & .00217289 & .3979300 \\
\hline & & & 16.3 & .00221305 & .4052258 \\
\hline & & & 16.4 & .00225394 & .4125998 \\
\hline & & & 16.5 & .00229557 & .4200522 \\
\hline & & & 16.6 & .00233795 & .4275832 \\
\hline & & & 16.7 & .00238109 & .4351925 \\
\hline & & & 16.8 & .00242499 & .4428801 \\
\hline & & & 16.9 & .00246967 & .4506458 \\
\hline & & & 17.0 & .00251512 & .4584892 \\
\hline & & & 17.1 & .00256137 & .4664099 \\
\hline & & & 17.2 & .00260841 & .4744074 \\
\hline & & & 17.3 & .00265625 & .4824812 \\
\hline & & & 17.4 & .00270491 & .4906307 \\
\hline & & & 17.5 & .00275438 & .4988551 \\
\hline & & & 17.6 & .00280468 & .5071538 \\
\hline & & & 17.7 & .00285581 & .5155260 \\
\hline & & & 17.8 & .00290779 & .5239710 \\
\hline & & & 17.9 & .00296061 & .5324879 \\
\hline 13.0 & .00123062 & .2033212 & 18.0 & .00301429 & .5410760 \\
\hline 13.1 & .00125120 & .2082522 & 18.1 & .00306883 & .5497344 \\
\hline 13.2 & .00127228 & .2132663 & 18.2 & .00312424 & .5584624 \\
\hline 13.3 & .00129386 & .2183622 & 18.3 & .00318052 & .5672591 \\
\hline 13.4 & .00131595 & .2235382 & 18.4 & .00323769 & .5761238 \\
\hline 13.5 & .00133857 & .2287929 & 18.5 & .00329575 & .5850558 \\
\hline 13.6 & .00136171 & .2341247 & 18.6 & .00335470 & .5940542 \\
\hline 13.7 & .00138539 & .2395323 & 18.7 & .00341456 & .6031185 \\
\hline 13.8 & .00140962 & .2450143 & 18.8 & .00347533 & .6122479 \\
\hline 13.9 & .00143440 & .2505696 & 18.9 & .00353701 & .6214418 \\
\hline 14.0 & .00145974 & .2561970 & 19.0 & .00359962 & .6306996 \\
\hline 14.1 & .00148564 & .2618957 & 19.1 & .00366316 & .6400207 \\
\hline 14.2 & .00151212 & .2676649 & 19.2 & .00372763 & .6494046 \\
\hline 14.3 & .00153918 & .2735041 & 19.3 & .00379304 & .6588507 \\
\hline 14.4 & .00156682 & .2794127 & 19.4 & .00385940 & .6683587 \\
\hline 14.5 & .00159506 & .2853906 & 19.5 & .00392671 & .6779280 \\
\hline 14.6 & .00162390 & .2914375 & 19.6 & .00399499 & .6875581 \\
\hline 14.7 & .00165335 & .2975537 & 19.7 & .00406423 & .6972487 \\
\hline 14.8 & .00168342 & .3037392 & 19.8 & .00413444 & .7069993 \\
\hline 14.9 & .00171410 & .3099944 & 19.9 & .00420563 & .7168096 \\
\hline 15.0 & .00174542 & .3163198 & 20.0 & .00427780 & .7266791 \\
\hline
\end{tabular}


IPTS-68 Reference Function and Its Derivative With the Argument in Kelvin-Continued

\begin{tabular}{|c|c|c|c|c|c|}
\hline $\begin{array}{l}T \\
\mathrm{~K}\end{array}$ & $W^{*}(T)$ & $\begin{array}{c}d W^{*}(T) / d T \\
\times 1000 \\
\mathrm{~K}^{-1}\end{array}$ & $\begin{array}{l}T \\
\mathrm{~K}\end{array}$ & $W^{*}(T)$ & $\begin{array}{c}d W^{*}(T) / d T \\
\times 10 \%(1) \\
\mathrm{K}^{-1}\end{array}$ \\
\hline 20.0 & .00427780 & .7266791 & 25.0 & .00925506 & 1.2810697 \\
\hline 20.1 & .00435097 & .7366074 & 25.1 & .00938376 & 1. 2929735 \\
\hline 20.2 & .00442513 & .7465942 & 25.2 & .00951365 & 1.3048932 \\
\hline 20.3 & .00450029 & .7566391 & 25.3 & .00964474 & 1.3168281 \\
\hline 20.4 & .00457646 & .7667415 & 25.4 & .00977702 & 1.3287776 \\
\hline 20.5 & .00465364 & .7769012 & 25.5 & .00991049 & 1.3407410 \\
\hline 20.6 & .00473184 & .7871176 & 25.6 & .01004517 & 1.3527177 \\
\hline 20.7 & .00481106 & .7973902 & 25.7 & 01018104 & 1.3647070 \\
\hline 20.8 & .00489132 & .8077186 & 25.8 & .01031811 & 1.3767085 \\
\hline 20.9 & .00497261 & .8181023 & 25.9 & .01045638 & 1.3887215 \\
\hline 21.0 & .00505494 & .8285406 & 26.0 & 01059585 & 1.4007456 \\
\hline 21.1 & .00513832 & 8390330 & 26.1 & .01073653 & 1.4127801 \\
\hline 21.2 & .00522275 & .8495789 & 26.2 & .01087841 & 1.4248246 \\
\hline 21.3 & .00530824 & .8601776 & 26.3 & .01102149 & 1.4368786 \\
\hline 21.4 & .00539479 & .8708284 & 26.4 & .01116578 & 1.4489416 \\
\hline 21.5 & .00548240 & .8815306 & 26.5 & .01131128 & 1.4610132 \\
\hline 21.6 & .00557109 & .8922834 & 26.6 & .01145799 & 1.4730930 \\
\hline 21.7 & .00566086 & .9030861 & 26.7 & .01160590 & 1.4851804 \\
\hline 21.8 & .00575171 & .9139377 & 26.8 & .01175502 & 1.4972751 \\
\hline 21.9 & .00584365 & .9248375 & 26.9 & .01190536 & 1.5093766 \\
\hline 22.0 & .00593668 & .9357846 & 27.0 & .01205690 & 1.5214845 \\
\hline 22.1 & .00603081 & .9467780 & 27.1 & .01220965 & 1.5335984 \\
\hline 22.2 & .00612604 & .9578167 & 27.2 & .01236362 & 1.5457180 \\
\hline 22.3 & .00622237 & .9688998 & 27.3 & .01251880 & 1.5578427 \\
\hline 22.4 & .00631982 & .9800263 & 27.4 & .01267519 & 1.5699723 \\
\hline 22.5 & .00641838 & 9911951 & 27.5 & .01283279 & 1.5821063 \\
\hline 22.6 & .00651806 & 1.0024053 & 27.6 & .01299161 & 1.5942443 \\
\hline 22.7 & .00661886 & 1.0136557 & 27.7 & .01315164 & 1.6063859 \\
\hline 22.8 & .00672079 & 1.0249453 & 27.8 & .01331289 & 1.6185307 \\
\hline 22.9 & .00682385 & 1.0362729 & 27.9 & .01347535 & 1.6306783 \\
\hline 23.0 & .00692805 & 1.0476376 & 28.0 & .01363902 & 1.6428283 \\
\hline 23.1 & .00703338 & 1.0590382 & 28.1 & 01380391 & 1.6549803 \\
\hline 23.2 & .00713986 & 1.0704735 & 28.2 & .01397002 & 1.6671338 \\
\hline 23.3 & .00724748 & 1.0819427 & 28.3 & .01413734 & 1.6792885 \\
\hline 23.4 & .00735625 & 1.0934444 & 28.4 & .01430588 & 1.6914438 \\
\hline 23.5 & .00746617 & 1.1049777 & 28.5 & .01447563 & 1.7035995 \\
\hline 23.6 & .00757724 & 1.1165414 & 28.6 & .01464660 & 1.7157549 \\
\hline 23.7 & .00768948 & 1.1281345 & 28.7 & .01481878 & 1.7279097 \\
\hline 23.8 & .00780287 & 1.1397560 & 28.8 & .01499218 & 1.7400633 \\
\hline 23.9 & .00791743 & 1.1514048 & 28.9 & .01516679 & 1.7522154 \\
\hline 24.0 & .00803315 & 1.1630800 & 29.0 & .01534262 & 1.7643654 \\
\hline 24.1 & .00815005 & 1.1747804 & 29.1 & .01551966 & 1.7765129 \\
\hline 24.2 & .00826811 & 1.1865051 & 29.2 & .01569792 & 1.7886573 \\
\hline 24.3 & .00838735 & 1.1982532 & 29.3 & .01587740 & 1.8007982 \\
\hline 24.4 & .00850776 & 1.2100238 & 29.4 & .01605808 & 1.8129350 \\
\hline 24.5 & 00862935 & 1.2218159 & 29.5 & .01623998 & 1.8250672 \\
\hline 24.6 & .00875213 & 1. 2336287 & 29.6 & .01642310 & 1.8371944 \\
\hline 24.7 & .00887608 & 1.2454613 & 29.7 & .01660742 & 1.8493158 \\
\hline 24.8 & .00900122 & 1.2573128 & 29.8 & .01679296 & 1.8614311 \\
\hline 24.9 & .00912754 & 1.2691826 & 29.9 & .01697971 & 1.8735397 \\
\hline 25.0 & .00925506 & 1.2810697 & 30.0 & .01716767 & 1.8856409 \\
\hline
\end{tabular}


IPTS-68 Reference Function and Its Derivative With the Argument in Kelvin-Continued

\begin{tabular}{|c|c|c|c|c|c|}
\hline $\begin{array}{l}T \\
\mathrm{~K}\end{array}$ & $W^{*}(T)$ & $\begin{array}{c}d W^{*}(T) / d T \\
\times \operatorname{K}^{-1}\end{array}$ & $\begin{array}{l}T \\
\mathbf{K}\end{array}$ & $W^{*}(T)$ & $\begin{array}{c}d W^{*}(T) / d T \\
\times 1000 \\
\mathbf{K}^{-1}\end{array}$ \\
\hline 30.0 & .01716767 & 1.8856409 & 35.0 & .02807645 & 2.4686391 \\
\hline 30.1 & .01735684 & 1.8977343 & 35.1 & .02832386 & 2.4796248 \\
\hline 30.2 & .01754721 & 1.9098192 & 35.2 & .02857237 & 2.4905766 \\
\hline 30.3 & .01773880 & 1.9218951 & 35.3 & .02882197 & 2.5014945 \\
\hline 30.4 & .01793159 & 1.9339615 & 35.4 & .02907267 & 2.5123779 \\
\hline 30.5 & .01812559 & 1.9460176 & 35.5 & .02932445 & 2.5232267 \\
\hline 30.6 & .01832080 & 1.9580630 & 35.6 & .02957731 & 2.5340406 \\
\hline 30.7 & .01851720 & 1.9700970 & 35.7 & 02983125 & 2.5448194 \\
\hline 30.8 & .01871481 & 1.9821191 & 35.8 & 03008627 & 2.5555628 \\
\hline 30.9 & 01891363 & 1.9941286 & 35.9 & .03034237 & 2.5662706 \\
\hline 31.0 & 01911364 & 2.0061250 & 36.0 & .03059953 & 2.5769426 \\
\hline 31.1 & .01931485 & 2.0181076 & 36.1 & .03085775 & 2.5875786 \\
\hline 31.2 & .01951726 & 2.0300758 & 36.2 & .03111704 & 2.5981783 \\
\hline 31.3 & .01972087 & 2.0420291 & 36.3 & .03137739 & 2.6087417 \\
\hline 31.4 & .01992567 & 2.0539668 & 36.4 & .03163879 & 2.6192684 \\
\hline 31.5 & .02013166 & 2.0658884 & 36.5 & .03190124 & 2.6297583 \\
\hline 31.6 & .02033884 & 2.0777932 & 36.6 & .03216474 & 2.6402113 \\
\hline 31.7 & .02054722 & 2.0896806 & 36.7 & .03242928 & 2.6506273 \\
\hline 31.8 & .02075678 & 2.1015501 & 36.8 & .03269486 & 2.6610059 \\
\hline 31.9 & .02096753 & 2.1134010 & 36.9 & .03296148 & 2.6713472 \\
\hline 32.0 & .02117946 & 2.1252328 & 37.0 & .03322913 & 2.6816510 \\
\hline 32.1 & .02139257 & 2.1370449 & 37.1 & .03349781 & 2.6919171 \\
\hline 32.2 & .02160687 & 2.1488366 & 37.2 & .03376751 & 2.7021455 \\
\hline 32.3 & .02182234 & 2.1606075 & 37.3 & .03403824 & 2.7123360 \\
\hline 32.4 & .02203899 & 2.1723570 & 37.4 & .03430998 & 2.7224886 \\
\hline 32.5 & .02225681 & 2.1840844 & 37.5 & .03458273 & 2.7326031 \\
\hline 32.6 & .02247580 & 2.1957893 & 37.6 & 03485650 & 2.7426794 \\
\hline 32.7 & .02269597 & 2.2074710 & 37.7 & .03513127 & 2.7527175 \\
\hline 32.8 & .02291730 & 2.2191291 & 37.8 & .03540704 & 2.7627173 \\
\hline 32.9 & .02313979 & 2.2307631 & 37.9 & .03568381 & 2.7726787 \\
\hline 33.0 & .02336345 & 2.2423723 & 38.0 & .03596158 & 2.7826016 \\
\hline 33.1 & .02358827 & 2.2539562 & 38.1 & .03624033 & 2.7924860 \\
\hline 33.2 & .02381424 & 2.2655145 & 38.2 & .03652007 & 2.8023319 \\
\hline 33.3 & .02404137 & 2.2770464 & 38.3 & .03680080 & 2.8121391 \\
\hline 33.4 & .02426965 & 2.2885517 & 38.4 & .03708250 & 2.8219077 \\
\hline 33.5 & .02449908 & 2.3000297 & 38.5 & .03736518 & 2.8316376 \\
\hline 33.6 & .02472965 & 2.3114801 & 38.6 & .03764882 & 2.8413287 \\
\hline 33.7 & .02496137 & 2.3229023 & 38.7 & .03793344 & 2.8509810 \\
\hline 33.8 & .02519423 & 2.3342959 & 38.8 & .03821902 & 2.8605946 \\
\hline 33.9 & .02542823 & 2.3456605 & 38.9 & .03850556 & 2.8701693 \\
\hline 34.0 & .02566336 & 2.3569956 & 39.0 & .03879305 & 2.8797052 \\
\hline 34.1 & .02589963 & 2.3683009 & 39.1 & .03908150 & 2.8892022 \\
\hline 34.2 & .02613702 & 2.3795758 & 39.2 & .03937089 & 2.8986603 \\
\hline 34.3 & .02637554 & 2.3908200 & 39.3 & .03966123 & 2.9080795 \\
\hline 34.4 & .02661518 & 2.4020332 & 39.4 & .03995251 & 2.9174598 \\
\hline 34.5 & .02685595 & 2.4132149 & 39.5 & .04024472 & 2.9268012 \\
\hline 34.6 & .02709783 & 2.4243648 & 39.6 & .04053786 & 2.9361038 \\
\hline 34.7 & .02734082 & 2.4354825 & 39.7 & .04083194 & 2.9453674 \\
\hline 34.8 & .02758492 & 2.4465677 & 39.8 & .04112694 & 2.9545921 \\
\hline 34.9 & .02783013 & 2.4576200 & 39.9 & .04142286 & 2.9637779 \\
\hline 35.0 & .02807645 & 2.4686391 & 40.0 & .04171969 & 2.9729248 \\
\hline
\end{tabular}


IPTS-68 Reference Function and Its Derivative With the Argument in Kelvin-Continued

\begin{tabular}{|c|c|c|c|c|c|}
\hline $\begin{array}{l}T \\
\mathbf{K}\end{array}$ & $W^{*}(T)$ & $\begin{array}{c}d W^{*}(T) / d T \\
\times 1000 \\
\mathrm{~K}^{-1} \\
\end{array}$ & $\begin{array}{l}\boldsymbol{T} \\
\mathbf{K}\end{array}$ & $W^{*}(T)$ & $\begin{array}{c}d W^{*}(T) / d T \\
\times 1000 \\
K^{-1}\end{array}$ \\
\hline 40.0 & .04171969 & 2.9729248 & 45.0 & .05764487 & 3.3812367 \\
\hline 40.1 & .04201744 & 2.9820329 & 45.1 & .05798335 & 3.3884379 \\
\hline 40.2 & .04231610 & 2.9911020 & 45.2 & .05832255 & 3.3956019 \\
\hline 40.3 & .04261566 & 3.0001324 & 45.3 & .05866247 & 3.4027289 \\
\hline 40.4 & .04291612 & 3.0091238 & 45.4 & .05900310 & 3.4098188 \\
\hline 40.5 & .04321748 & 3.0180765 & 45.5 & .05934443 & 3.4168718 \\
\hline 40.6 & .04351974 & 3.0269903 & 45.6 & .05968647 & 3.4238879 \\
\hline 40.7 & .04382288 & 3.0358654 & 45.7 & .06002921 & 3.4308672 \\
\hline 40.8 & .04412691 & 3.0447017 & 45.8 & .06037264 & 3.4378096 \\
\hline 40.9 & .04443182 & 3.0534993 & 45.9 & .06071677 & 3.4447153 \\
\hline 41.0 & .04473761 & 3.0622582 & 46.0 & .06106158 & 3.4515843 \\
\hline 41.1 & .04504427 & 3.0709783 & 46.1 & .06140708 & 3.4584167 \\
\hline 41.2 & .04535180 & 3.0796598 & 46.2 & .06175327 & $3.465212 j$ \\
\hline 41.3 & .04566020 & 3.0883026 & 46.3 & .06210013 & 3.4719718 \\
\hline 41.4 & .04596946 & 3.0969069 & 46.4 & .06244766 & 3.4786946 \\
\hline 41.5 & .04627958 & 3.1054726 & 46.5 & .06279586 & 3.4853811 \\
\hline 41.6 & .04659055 & 3. 1139997 & 46.6 & .06314473 & 3.4920311 \\
\hline 41.7 & .04690238 & 3.1224883 & 46.7 & .06349427 & 3.4986449 \\
\hline 41.8 & .04721505 & 3.1309384 & 46.8 & .06384446 & 3.5052225 \\
\hline 41.9 & .04752856 & 3.1393500 & 46.9 & .06419531 & 3.5117639 \\
\hline 42.0 & .04784292 & 3.1477233 & 47.0 & .06454681 & 3.5182692 \\
\hline 42.1 & .04815811 & 3.1560581 & 47.1 & .06489896 & 3.5247385 \\
\hline 42.2 & .04847413 & 3. 1643546 & 47.2 & .06525176 & 3.5311718 \\
\hline 42.3 & .04879098 & 3.1726127 & 47.3 & .06560520 & 3.5375693 \\
\hline 42.4 & .04910865 & 3. 1808326 & 47.4 & .06595927 & 3.5439308 \\
\hline 42.5 & .04942714 & 3. 1890142 & 47.5 & .06631398 & 3.5502567 \\
\hline 42.6 & .04974645 & 3.1971576 & 47.6 & .06666932 & 3.5565468 \\
\hline 42.7 & .05006657 & 3.2052628 & 47.7 & .06702529 & 3.5628013 \\
\hline 42.8 & .05038750 & 3.2133299 & 47.8 & .06738188 & 3.5690202 \\
\hline 42.9 & .05070924 & 3.2213589 & 47.9 & .06773909 & 3.5752037 \\
\hline 43.0 & .05103177 & 3.2293498 & 48.0 & .06809692 & 3.5813517 \\
\hline 43.1 & .05135511 & 3.2373027 & 48.1 & .06845536 & 3.5874645 \\
\hline 43.2 & .05167923 & 3.2452176 & 48.2 & .06881441 & 3.5935419 \\
\hline 43.3 & .05200415 & 3.2530945 & 48.3 & .06917407 & 3.5995842 \\
\hline 43.4 & .05232985 & 3.2609335 & 48.4 & .06953433 & 3.6055913 \\
\hline 43.5 & .05265633 & 3.2687347 & 48.5 & .06989519 & 3.6115635 \\
\hline 43.6 & .05298359 & 3.2764980 & 48.6 & .07025664 & 3.6175007 \\
\hline 43.7 & .05331163 & 3.2842236 & 48.7 & .07061869 & 3.6234030 \\
\hline 43.8 & .05364044 & 3.2919114 & 48.8 & 07098132 & 3.6292705 \\
\hline 43.9 & .05397001 & 3.2995615 & 48.9 & 07134454 & 3.6351033 \\
\hline 44.0 & .05430035 & 3.3071739 & 49.0 & .07170834 & 3.6409015 \\
\hline 44.1 & .05463145 & 3.3147488 & 49.1 & .07207272 & 3.6466652 \\
\hline 44.2 & .05496330 & 3.3222860 & 49.2 & .07243767 & 3.6523943 \\
\hline 44.3 & .05529590 & 3. 3297857 & 49.3 & .07280320 & 3.6580892 \\
\hline 44.4 & .05562925 & 3.3372479 & 49.4 & .07316929 & 3.6637497 \\
\hline 44.5 & .05596335 & 3.3446727 & 49.5 & .07353595 & 3.6693761 \\
\hline 44.6 & .05629819 & 3.3520602 & 49.6 & .07390316 & 3.6749683 \\
\hline 44.7 & .05663376 & 3.3594102 & 49.7 & .07427094 & 3.6805266 \\
\hline 44.8 & .05697007 & 3.3667230 & 49.8 & .07463927 & 3.6860509 \\
\hline 44.9 & .05730710 & 3.3739985 & 49.9 & .07500815 & 3.6915414 \\
\hline 45.0 & .05764487 & 3.3812367 & 50.0 & .07537757 & 3.6969982 \\
\hline
\end{tabular}


IPTS-68 Reference Function and Its Derivative With the Argument in Kelvin-Coptinued

\begin{tabular}{|c|c|c|c|c|c|}
\hline $\begin{array}{l}T \\
\mathbf{K}\end{array}$ & $W^{*}(T)$ & $\begin{array}{c}d W^{*}(T) / d T \\
\times \quad 1000 \\
K^{-1}\end{array}$ & $\begin{array}{l}\boldsymbol{T} \\
\mathbf{K}\end{array}$ & $W^{*}(T)$ & $\begin{array}{c}d W^{*}(T) / d T \\
\times 1000 \\
K^{-1}\end{array}$ \\
\hline 50.0 & .07537757 & 3.6969982 & 55.0 & .09447519 & 3.9291588 \\
\hline 50.1 & .07574755 & 3.7024213 & 55.1 & .09486830 & 3.9330396 \\
\hline 50.2 & .07611806 & 3.7078109 & 55.2 & .09526179 & 3.9368927 \\
\hline 50.3 & .07648911 & 3.7131670 & 55.3 & .09565568 & 3.9407182 \\
\hline 50.4 & .07686069 & 3.7184898 & 55.4 & .09604994 & 3.9445161 \\
\hline 50.5 & .07723280 & 3.7237793 & 55.5 & .09644458 & 3.9482867 \\
\hline 50.6 & .07760544 & 3.7290357 & 55.6 & .09683959 & 3.9520301 \\
\hline 50.7 & .07797861 & 3.7342591 & 55.7 & .09723498 & 3.9557464 \\
\hline 50.8 & .07835229 & 3.7394494 & 55.8 & .09763074 & 3.9594356 \\
\hline 50.9 & .07872650 & 3.7446070 & 55.9 & .09802687 & 3.9630981 \\
\hline 51.0 & .07910122 & 3.7497318 & 56.0 & .09842336 & 3.9667339 \\
\hline 51.1 & .07947644 & 3.7548239 & 56.1 & .09882022 & 3.9703430 \\
\hline 51.2 & .07985218 & 3.7598835 & 56.2 & .09921743 & 3.9739257 \\
\hline 51.3 & .08022842 & 3.7649107 & 56.3 & .09961500 & 3.9774821 \\
\hline 51.4 & .08060516 & 3.7699056 & 56.4 & .10001292 & 3.9810123 \\
\hline 51.5 & .08098240 & 3.7748682 & 56.5 & .10041120 & 3.9845165 \\
\hline 51.6 & .08136013 & 3.7797987 & 56.6 & .10080983 & 3.9879947 \\
\hline 51.7 & .08173836 & 3.7846973 & 56.7 & .10120880 & 3.9914472 \\
\hline 51.8 & .08211707 & 3.7895640 & 56.8 & .10160812 & 3.9948740 \\
\hline 51.9 & .08249627 & 3.7943988 & 56.9 & .10200777 & 3.9982753 \\
\hline 52.0 & .08287595 & 3.7992021 & 57.0 & .10240777 & 4.0016511 \\
\hline 52.1 & .08325611 & 3.8039737 & 57.1 & 10280810 & 4.0050017 \\
\hline 52.2 & .08363674 & 3.8087140 & 57.2 & .10320877 & 4.0083273 \\
\hline 52.3 & .08401785 & 3.8134229 & 57.3 & .10360977 & 4.0116278 \\
\hline 52.4 & .08439943 & 3.8181006 & 57.4 & .10401109 & 4.0149033 \\
\hline 52.5 & .08478147 & 3.8227472 & 57.5 & .10441275 & 4.0181542 \\
\hline 52.6 & .08516398 & 3.8273628 & 57.6 & .10481472 & 4.0213805 \\
\hline 52.7 & .08554694 & 3.8319476 & 57.7 & .10521702 & 4.0245823 \\
\hline 52.8 & .08593036 & 3.8365016 & 57.8 & .10561964 & 4.0277598 \\
\hline 52.9 & .08631424 & 3.8410250 & 57.9 & .10602257 & 4.0309130 \\
\hline 53.0 & .08669857 & 3.8455179 & 58.0 & .10642582 & 4.0340422 \\
\hline 53.1 & .08708334 & 3.8499805 & 58.1 & .10682938 & 4.0371474 \\
\hline 53.2 & .08746856 & 3.8544127 & 58.2 & .10723325 & 4.0402288 \\
\hline 53.3 & .08785422 & 3.8588148 & 58.3 & .10763743 & 4.0432865 \\
\hline 53.4 & .08824032 & 3.8631869 & 58.4 & 10804191 & 4.0463206 \\
\hline 53.5 & .08862686 & 3.8675291 & 58.5 & .10844669 & 4.0493314 \\
\hline 53.6 & .08901383 & 3.8718415 & 58.6 & .10885177 & 4.0523188 \\
\hline 53.7 & .08940123 & 3.8761243 & 58.7 & .10925715 & 4.0552830 \\
\hline 53.8 & .08978905 & 3.8803775 & 58.8 & .10966283 & 4.0582243 \\
\hline 53.9 & .09017730 & 3.8846014 & 58.9 & .11006880 & 4.0611426 \\
\hline 54.0 & .09056597 & 3.8887959 & 59.0 & .11047506 & 4.0640381 \\
\hline 54.1 & .09095506 & 3.8929613 & 59.1 & . 11088160 & 4.0669110 \\
\hline 54.2 & .09134456 & 3.8970977 & 59.2 & .11128844 & 4.0697614 \\
\hline 54.3 & .09173448 & 3.9012052 & 59.3 & .11169555 & 4.0725893 \\
\hline 54.4 & .09212480 & 3.9052839 & 59.4 & .11210295 & 4.0753950 \\
\hline 54.5 & .09251554 & 3.9093339 & 59.5 & .11251063 & 4.0781786 \\
\hline 54.6 & .09290667 & 3.9133554 & 59.6 & .11291859 & 4.0809402 \\
\hline 54.7 & .09329821 & 3.9173485 & 59.7 & .11332682 & 4.0836798 \\
\hline 54.8 & .09369014 & 3.9213134 & 59.8 & .11373532 & 4.0863978 \\
\hline 54.9 & .09408247 & 3.9252501 & 59.9 & .11414410 & 4.0890941 \\
\hline 55.0 & .09447519 & 3.9291588 & 60.0 & .11455314 & 4.0917688 \\
\hline
\end{tabular}


IPTS-68 Reference Funetion and Its Derivative With the Argument in Kelvin-Continued

\begin{tabular}{|c|c|c|c|c|c|}
\hline $\begin{array}{l}T \\
\mathbf{K}\end{array}$ & $W^{*}(T)$ & $\begin{array}{c}d W^{*}(T) / d T \\
\times \mathrm{j} 000 \\
\mathrm{~K}^{-1}\end{array}$ & $\begin{array}{l}T \\
\mathrm{~K}\end{array}$ & $W^{*}(T)$ & $\begin{array}{c}d W^{*}(T) / d T \\
\times 1000 \\
\mathbf{K}^{-1}\end{array}$ \\
\hline 60.0 & .11455314 & 4.0917688 & 65.0 & .13530364 & 4.2007677 \\
\hline 60.1 & .11496245 & 4.0944222 & 65.1 & .13572380 & 4.2025038 \\
\hline 60.2 & .11537203 & 4.0970544 & 65.2 & .13614414 & 4.2042243 \\
\hline 60.3 & .11578186 & 4.0996654 & 65.3 & .13656464 & 4.2059294 \\
\hline 60.4 & .11619196 & 4.1022555 & 65.4 & .13698532 & 4.2076192 \\
\hline 60.5 & .11660231 & 4. 1048246 & 65.5 & .13740617 & 4.2092937 \\
\hline 60.6 & .11701292 & 4.1073730 & 65.6 & .13782718 & 4.2109531 \\
\hline 60.7 & .11742379 & 4.1099008 & 65.7 & .13824836 & 4.2125975 \\
\hline 60.8 & .11783490 & 4.1124081 & 65.8 & .13866970 & 4.2142270 \\
\hline 60.9 & .11824627 & 4.1148950 & 65.9 & .13909120 & 4.2158416 \\
\hline 61.0 & .11865788 & 4.1173616 & 66.0 & .13951287 & 4.2174415 \\
\hline 61.1 & .11906974 & 4.1198081 & 66.1 & .13993469 & 4.2190268 \\
\hline 61.2 & .11948184 & 4.1222345 & 66.2 & .14035667 & 4.2205976 \\
\hline 61.3 & .11989419 & 4.1246411 & 66.3 & .14077881 & 4.2221539 \\
\hline 61.4 & .12030677 & 4.1270279 & 66.4 & .14120110 & 4.2236958 \\
\hline 61.5 & .12071959 & 4.1293951 & 66.5 & .14162355 & 4.2252236 \\
\hline 61.6 & .12113265 & 4.1317428 & 66.6 & .14204615 & 4.2267371 \\
\hline 61.7 & .12154594 & 4.1340709 & 66.7 & .14246889 & 4.2282367 \\
\hline 61.8 & .12195946 & 4.1363799 & 66.8 & .14289179 & 4.2297 .223 \\
\hline 61.9 & .12237321 & 4.1386696 & 66.9 & .14331484 & 4.2311939 \\
\hline 62.0 & .12278719 & 4.1409403 & 67.0 & .14373803 & 4.2326518 \\
\hline 62.1 & .12320140 & 4.1431921 & 67.1 & .14416137 & 4.2340961 \\
\hline 62.2 & .12361583 & 4.1454250 & 67.2 & 14458485 & 4.2355267 \\
\hline 62.3 & .12403049 & 4.1476393 & 67.3 & .14500847 & 4.2369439 \\
\hline 62.4 & .12444536 & 4.1498349 & 67.4 & .14543224 & 4.2383476 \\
\hline 62.5 & .12486045 & 4.1520121 & 67.5 & .14585614 & 4.2397380 \\
\hline 62.6 & .12527576 & 4.1541709 & 67.6 & .14628019 & 4.2411152 \\
\hline 62.7 & 12569129 & 4.1563115 & 67.7 & .14670436 & 4.2424793 \\
\hline 62.8 & .12610702 & 4.1584339 & 67.8 & .14712868 & 4.2438303 \\
\hline 62.9 & .12652297 & 4.1605384 & 67.9 & .14755313 & 4.2451683 \\
\hline 63.0 & .12693913 & 4.1626249 & 68.0 & .14797771 & 4.2464935 \\
\hline 63.1 & .12735550 & 4.1646937 & 68.1 & .14840243 & 4.2478058 \\
\hline 63.2 & .12777207 & 4.1667448 & 68.2 & .14882727 & 4.2491055 \\
\hline 63.3 & .12818884 & 4.1687783 & 68.3 & .14925225 & 4.2503925 \\
\hline 63.4 & .12860582 & 4.1707944 & 68.4 & .14967735 & 4.2516671 \\
\hline 63.5 & .12902300 & 4.1727931 & 68.5 & .15010258 & 4.2529291 \\
\hline 63.6 & .12944038 & 4.1747746 & 68.6 & .15052794 & 4. 2541788 \\
\hline 63.7 & .12985796 & 4.1767390 & 68.7 & .15095342 & 4.2554163 \\
\hline 63.8 & .13027573 & 4.1786864 & 68.8 & .15137902 & 4.2566415 \\
\hline 63.9 & 13069369 & 4. 1806169 & 68.9 & .15180475 & 4.2578547 \\
\hline 64.0 & .13111185 & 4.1825306 & 69.0 & .15223059 & 4.2590558 \\
\hline 64.1 & .33153020 & 4.1844276 & 69.1 & .15265656 & 4.2602449 \\
\hline 64.2 & .13194874 & 4.1863081 & 69.2 & .15308264 & 4.2614222 \\
\hline 64.3 & 13236746 & 4.1881721 & 69.3 & .15350884 & 4.2625877 \\
\hline 64.4 & 13278637 & 4. 1900197 & 69.4 & 15393516 & 4. 2637415 \\
\hline 64.5 & 13320546 & 4.1918511 & 69.5 & .15436159 & 4.2648838 \\
\hline 64.6 & .13362474 & 4.1936663 & 69.6 & .15478813 & 4.2660144 \\
\hline 64.7 & 13404420 & 4.1954655 & 69.7 & .15521479 & 4.2671336 \\
\hline 64.8 & 13446383 & 4.1972487 & 69.8 & 15564156 & 4.2682414 \\
\hline 64.9 & 13488365 & 4.1990161 & 69.9 & .15606844 & 4.2693379 \\
\hline 65.0 & 13530364 & 4.2007677 & 70.0 & .15649543 & 4.2704231 \\
\hline
\end{tabular}


IPTS-68 Reference Function and Its Derivative With the Argument in Kelvin-Continued

\begin{tabular}{|c|c|c|c|c|c|}
\hline $\begin{array}{l}T \\
\mathbf{K}\end{array}$ & $W^{*}(T)$ & $\begin{array}{c}d W^{*}(T) / d T \\
\times 1000 \\
K^{-1}\end{array}$ & $\begin{array}{l}T \\
\mathrm{~K}\end{array}$ & $W^{*}(T)$ & $\begin{array}{c}d W^{*}(T) / d T \\
\times 1000 \\
\mathbf{K}^{-1}\end{array}$ \\
\hline 70.0 & 15649543 & 4.2704231 & 75.0 & .17796116 & 4.3119570 \\
\hline 70.1 & .15692252 & 4.2714973 & 75.1 & .17839239 & 4.3125623 \\
\hline 70.2 & .15734973 & 4.2725603 & 75.2 & .17882367 & 4.3131598 \\
\hline 70.3 & .15777703 & 4.2736124 & 75.3 & .17925502 & 4.3137496 \\
\hline 70.4 & 15820445 & 4.2746535 & 75.4 & .17968642 & 4.3143316 \\
\hline 70.5 & 15863197 & 4.2756838 & 75.5 & .18011789 & 4.3149061 \\
\hline 70.6 & 15905958 & 4.2767033 & 75.6 & .18054940 & 4.3154730 \\
\hline 70.7 & 15948731 & 4.2777121 & 75.7 & . 18098098 & 4.3160323 \\
\hline 70.8 & 15991513 & 4.2787103 & 75.8 & .18141261 & 4.3165842 \\
\hline 70.9 & 16034305 & 4.2796980 & 75.9 & .18184430 & 4.3171287 \\
\hline 71.0 & 16077107 & 4.2806751 & 76.0 & .18227604 & 4.3176658 \\
\hline 71.1 & 16119918 & 4.2816419 & 76.1 & .18270783 & 4.3181957 \\
\hline 71.2 & 16162739 & 4.2825983 & 76.2 & .18313968 & 4.3187183 \\
\hline 71.3 & 16205570 & 4.2835445 & 76.3 & .18357157 & 4.3192336 \\
\hline 71.4 & 16248410 & 4.2844805 & 76.4 & .18400352 & 4.3197419 \\
\hline 71.5 & 16291260 & 4.2854064 & 76.5 & .18443552 & 4.3202431 \\
\hline 71.6 & 16334118 & 4.2863222 & 76.6 & .18486757 & 4.3207372 \\
\hline 71.7 & 16376986 & 4.2872280 & 76.7 & .18529967 & 4.3212243 \\
\hline 71.8 & .16419863 & 4.2881240 & 76.8 & .18573181 & 4.3217045 \\
\hline 71.9 & .16462749 & 4.2890101 & 76.9 & 18616401 & 4.3221778 \\
\hline 72.0 & .16505643 & 4.2898864 & 77.0 & .18659625 & 4.3226442 \\
\hline 72.1 & .16548546 & 4.2907530 & 77.1 & 18702854 & 4.3231039 \\
\hline 72.2 & .16591458 & 4.2916101 & 77.2 & .18746087 & 4.3235568 \\
\hline 72.3 & .16634378 & 4.2924575 & 77.3 & .18789325 & 4.3240030 \\
\hline 72.4 & .16677307 & 4.2932954 & 77.4 & .18832567 & 4.3244426 \\
\hline 72.5 & .16720244 & 4.2941240 & 77.5 & .18875814 & 4.3248755 \\
\hline 72.6 & .16763190 & 4.2949431 & 77.6 & .18919065 & 4.3253020 \\
\hline 72.7 & .16806143 & 4.2957529 & 77.7 & .18962320 & 4.3257219 \\
\hline 72.8 & .16849105 & 4.2965535 & 77.8 & .19005579 & 4.3261354 \\
\hline 72.9 & .16892074 & 4.2973449 & 77.9 & 19048842 & 4.3265424 \\
\hline 73.0 & .16935052 & 4.2981273 & 78.0 & .19092110 & 4.3269431 \\
\hline 73.1 & .16978037 & 4.2989005 & 78.1 & .19135381 & 4.3273375 \\
\hline 73.2 & .17021030 & 4.2996649 & 78.2 & 19178657 & 4.3277257 \\
\hline 73.3 & .17064030 & 4.3004202 & 78.3 & .19221936 & 4.3281076 \\
\hline 73.4 & .17107038 & 4.3011667 & 78.4 & .19265219 & 4.3284832 \\
\hline 73.5 & .17150053 & 4.3019045 & 78.5 & .19308505 & 4. 3288529 \\
\hline 73.6 & .17193076 & 4.3026335 & 78.6 & .19351796 & 4.3292164 \\
\hline 73.7 & .17236106 & 4.3033539 & 78.7 & 19395090 & 4.3295738 \\
\hline 73.8 & .17279143 & 4.3040656 & 78.8 & 19438387 & 4. 3299253 \\
\hline 73.9 & .17322187 & 4.3047688 & 78.9 & 19481688 & 4.3302707 \\
\hline 74.0 & .17365238 & 4.3054635 & 79.0 & 19524993 & 4.3306104 \\
\hline 74.1 & .17408296 & 4.3061498 & 79.1 & 19568300 & 4.3309441 \\
\hline 74.2 & .17451361 & 4.3068277 & 79.2 & .19611611 & 4.3312720 \\
\hline 74.3 & .17494433 & 4.3074974 & 79.3 & .19654926 & 4.3315941 \\
\hline 74.4 & .17537511 & 4.3081587 & 79.4 & 19698243 & 4.3319105 \\
\hline 74.5 & .17580596 & 4.3088119 & 79.5 & 19741564 & 4.3322212 \\
\hline 74.6 & .17623687 & 4.3094570 & 79.6 & 19784888 & 4. 3325262 \\
\hline 74.7 & 17666785 & 4.3100939 & 79.7 & .19828214 & 4.3328256 \\
\hline 74.8 & 17709889 & 4.3107229 & 79.8 & .19871544 & 4.3331194 \\
\hline 74.9 & .17753000 & 4.3113439 & 79.9 & .19914877 & 4.3334077 \\
\hline 75.0 & 17796116 & 4.3119570 & 80.0 & 19958212 & 4.3336906 \\
\hline
\end{tabular}


IPTS-68 Reference Function and Its Derivative With the Argument in Kelvin-Continued

\begin{tabular}{|c|c|c|c|c|c|}
\hline $\begin{array}{l}T \\
\mathbf{K}\end{array}$ & $W^{*}(T)$ & $\begin{array}{c}d W^{*}(T) / d T \\
\times 1000 \\
\mathrm{~K}^{-1} \\
\end{array}$ & $\begin{array}{l}T \\
\mathrm{~K}\end{array}$ & $W^{*}(T)$ & $\begin{array}{c}d W^{*}(T) / d T \\
\times 1000 \\
K^{-1} \\
\end{array}$ \\
\hline 80.0 & .19958212 & 4.3336906 & 85.0 & .22127523 & 4.3416637 \\
\hline 80.1 & .20001551 & 4.3339679 & 85.1 & .22170939 & 4.3417150 \\
\hline 80.2 & .20044892 & 4.3342399 & 85.2 & .22214357 & 4.3417627 \\
\hline 80.3 & .20088235 & 4.3345065 & 85.3 & .22257775 & 4.3418067 \\
\hline 80.4 & .20131582 & 4.3347678 & 85.4 & .22301193 & 4.3418471 \\
\hline 80.5 & .20174931 & 4.3350238 & 85.5 & .22344612 & 4.3418839 \\
\hline 80.6 & .20218282 & 4.3352745 & 85.6 & .22388031 & 4.3419171 \\
\hline 80.7 & .20261636 & 4.3355200 & 85.7 & .22431450 & 4.3419467 \\
\hline 80.8 & .20304993 & 4.3357603 & 85.8 & .22474870 & 4. 3419728 \\
\hline 80.9 & .20348351 & 4.3359955 & 85.9 & .22518289 & 4.3419955 \\
\hline 81.0 & .20391713 & 4.3362257 & 86.0 & .22561709 & 4.3420146 \\
\hline 81.1 & .20435076 & 4.3364507 & 86.1 & .22605130 & 4.3420303 \\
\hline 81.2 & .20478442 & 4.3366708 & 86.2 & .22648550 & 4.3420427 \\
\hline 81.3 & .20521809 & 4.3368858 & 86.3 & .22691971 & 4.3420516 \\
\hline 81.4 & .20565179 & 4.3370959 & 86.4 & .22735391 & 4.3420572 \\
\hline 81.5 & .20608551 & 4.3373012 & 86.5 & .22778812 & 4.3420594 \\
\hline 81.6 & .20651925 & 4.3375015 & 86.6 & .22822232 & 4.3420584 \\
\hline 81.7 & .20695301 & 4.3376970 & 86.7 & .22865653 & 4.3420541 \\
\hline 81.8 & .20738679 & 4.3378877 & 86.8 & 22909073 & 4.3420465 \\
\hline 81.9 & .20782059 & 4.3380737 & 86.9 & .22952494 & 4.3420357 \\
\hline 82.0 & .20825441 & 4.3382549 & 87.0 & .22995914 & 4.3420217 \\
\hline 82.1 & 20868824 & 4.3384315 & 87.1 & .23039334 & 4.3420045 \\
\hline 82.2 & .20912209 & 4.3386034 & 87.2 & .23082754 & 4.3419842 \\
\hline 82.3 & .20955596 & 4.3387706 & 87.3 & .23126174 & 4.3419608 \\
\hline 82.4 & .20998985 & 4.3389333 & 87.4 & .23169593 & 4.3419342 \\
\hline 82.5 & .21042375 & 4.3390914 & 87.5 & .23213013 & 4.3419047 \\
\hline 82.6 & .21085766 & 4.3392450 & 87.6 & .23256431 & 4.3418720 \\
\hline 82.7 & .21129160 & 4.3393942 & 87.7 & .23299850 & 4.3418363 \\
\hline 82.8 & .21172554 & 4.3395389 & 87.8 & .23343268 & 4.3417977 \\
\hline 82.9 & .21215950 & 4.3396791 & 87.9 & .23386686 & 4.3417560 \\
\hline 83.0 & .21259348 & 4.3398151 & 88.0 & .23430103 & 4.3417114 \\
\hline 83.1 & .21302747 & 4.3399466 & 88.1 & .23473520 & 4.3416639 \\
\hline 83.2 & .21346147 & 4.3400739 & 88.2 & 23516937 & 4.3416135 \\
\hline 83.3 & .21389548 & 4.3401968 & 88.3 & .23560352 & 4.3415602 \\
\hline 83.4 & .21432951 & 4.3403155 & 88.4 & .23603768 & 4.3415041 \\
\hline 83.5 & .21476354 & 4.3404301 & 88.5 & .23647182 & 4.3414451 \\
\hline 83.6 & .21519759 & 4.3405404 & 88.6 & .23690597 & 4.3413833 \\
\hline 83.7 & .21563165 & 4.3406466 & 88.7 & 23734010 & 4.3413188 \\
\hline 83.8 & .21606572 & 4.3407487 & 88.8 & .23777423 & 4.3412514 \\
\hline 83.9 & 21649980 & 4.3408466 & 88.9 & .23820835 & 4.3411814 \\
\hline 84.0 & 21693389 & 4.3409406 & 89.0 & 23864247 & 4.3411086 \\
\hline 84.1 & .21736799 & 4.3410304 & 89.1 & .23907657 & 4.3410332 \\
\hline 84.2 & 21780210 & 4.3411163 & 89.2 & .23951067 & 4. 3409551 \\
\hline 84.3 & .21823621 & 4.3411983 & 89.3 & .23994476 & 4.3408743 \\
\hline 84.4 & .21867034 & 4.3412764 & 89.4 & 24037885 & 4.3407909 \\
\hline 84.5 & .21910447 & 4.3413505 & 89.5 & .24081292 & 4.3407048 \\
\hline 84.6 & .21953861 & 4.3414207 & 89.6 & .24124699 & 4.3406162 \\
\hline 84.7 & .21997275 & 4.3414871 & 89.7 & .24168105 & 4.3405251 \\
\hline 84.8 & .22040690 & 4.3415498 & 89.8 & .24211509 & 4.3404314 \\
\hline 84.9 & .22084106 & 4.3416086 & 89.9 & .24254913 & 4.3403352 \\
\hline 85.0 & .22127523 & 4.3416637 & 90.0 & .24298316 & 4.3402365 \\
\hline
\end{tabular}


IPTS-68 Reference Function and Its Derivative With the Argument in Kelvin-Continued

\begin{tabular}{|c|c|c|c|c|c|}
\hline $\begin{array}{l}T \\
\mathbf{K}\end{array}$ & $W^{*}(T)$ & $\begin{array}{c}d W^{*}(T) / d T \\
\times 1000 \\
\mathrm{~K}^{-1}\end{array}$ & $\begin{array}{l}T \\
\mathrm{~K}\end{array}$ & $W^{*}(T)$ & $\begin{array}{c}d W^{*}(T) ! d T \\
\times 1000 \\
\mathrm{~K}^{-1}\end{array}$ \\
\hline 90.0 & .24298316 & 4.3402365 & 95.0 & .26466721 & 4.3325507 \\
\hline 90.1 & 24341718 & 4.3401353 & 95.1 & .26510045 & 4.3323498 \\
\hline 90.2 & 24385119 & 4.3400317 & 95.2 & .26553368 & 4.3321474 \\
\hline 90.3 & .24428519 & 4.3399257 & 95.3 & .26596688 & 4.3319435 \\
\hline 90.4 & .24471917 & 4.3398172 & 95.4 & 26640007 & 4.3317379 \\
\hline 90.5 & .24515315 & 4.3397063 & 95.5 & .26683323 & 4.3315309 \\
\hline 90.6 & .24558711 & 4.3395931 & 95.6 & .26726637 & 4.3313224 \\
\hline 90.7 & .24602107 & 4.3394775 & 95.7 & .26769950 & 4.3311124 \\
\hline 90.8 & .24645501 & 4.3393596 & 95.8 & .26813260 & 4.3309009 \\
\hline 90.9 & .24688894 & 4.3392394 & 95.9 & .26856568 & 4.3306879 \\
\hline 91.0 & .24732286 & 4.3391169 & 96.0 & .26899873 & 4.3304735 \\
\hline 91.1 & .24775676 & 4.3389921 & 96.1 & .26943177 & 4.3302577 \\
\hline 91.2 & .24819065 & 4.3388651 & 96.2 & .26986479 & 4.3300404 \\
\hline 91.3 & .24862454 & 4.3387358 & 96.3 & .27029778 & 4.3298217 \\
\hline 91.4 & 24905840 & 4.3386043 & 96.4 & .27073075 & 4.3296017 \\
\hline 91.5 & 24949226 & 4.3384706 & 96.5 & .27116370 & 4.3293803 \\
\hline 91.6 & .24992610 & 4.3383348 & 96.6 & .27159663 & 4.3291575 \\
\hline 91.7 & .25035992 & 4.3381968 & 96.7 & .27202953 & 4.3289333 \\
\hline 91.8 & .25079374 & 4.3380566 & 96.8 & .27246241 & 4.3287078 \\
\hline 91.9 & .25122753 & 4.3379143 & 96.9 & .27289527 & 4.3284810 \\
\hline 92.0 & 25166132 & 4.3377700 & 97.0 & .27332811 & 4.3282529 \\
\hline 92.1 & 25209509 & 4.3376236 & 97.1 & .27376092 & 4.3280234 \\
\hline 92.2 & .25252884 & 4.3374751 & 97.2 & .27419371 & 4.3277927 \\
\hline 92.3 & 25296258 & 4.3373245 & 97.3 & .27462648 & 4.3275607 \\
\hline 92.4 & 25339631 & 4.3371720 & 97.4 & .27505922 & 4.3273274 \\
\hline 92.5 & .25383002 & 4.3370174 & 97.5 & .27549195 & 4.3270929 \\
\hline 92.6 & 25426371 & 4.3368608 & 97.6 & .27592464 & 4.3268572 \\
\hline 92.7 & .25469739 & 4.3367023 & 97.7 & .27635732 & 4.3266203 \\
\hline 92.8 & .25513105 & 4.3365418 & 97.8 & 27678997 & 4.3263821 \\
\hline 92.9 & .25556470 & 4.3363794 & 97.9 & .27722259 & 4.3261427 \\
\hline 93.0 & .25599833 & 4.3362150 & 98.0 & .27765520 & 4.3259021 \\
\hline 93.1 & .25643194 & 4.3360487 & 98.1 & .27808777 & 4.3256604 \\
\hline 93.2 & .25686554 & 4.3358806 & 98.2 & .27852033 & 4.3254176 \\
\hline 93.3 & .25729912 & 4.3357106 & 98.3 & .27895286 & 4.3251735 \\
\hline 93.4 & .25773268 & 4.3355388 & 98.4 & .27938536 & 4.3249283 \\
\hline 93.5 & .25816622 & 4.3353651 & 98.5 & .27981784 & 4.3246821 \\
\hline 93.6 & .25859975 & 4.3351896 & 98.6 & .28025030 & 4.3244347 \\
\hline 93.7 & 25903326 & 4.3350122 & 98.7 & .28068273 & 4.3241862 \\
\hline 93.8 & 25946675 & 4.3348332 & 98.8 & .28111514 & 4.3239366 \\
\hline 93.9 & 25990023 & 4.3346523 & 98.9 & .28154752 & 4.3236859 \\
\hline 94.0 & 26033368 & 4.3344697 & 99.0 & .28197987 & 4.3234341 \\
\hline 94.1 & .26076712 & 4.3342853 & 99.1 & .28241220 & 4.3231813 \\
\hline 94.2 & .26120054 & 4.3340992 & 99.2 & .28284451 & 4.3229276 \\
\hline 94.3 & .26163394 & 4.3339115 & 99.3 & .28327679 & 4.3226727 \\
\hline 94.4 & .26206732 & 4.3337220 & 99.4 & .28370904 & 4.3224168 \\
\hline 94.5 & .26250069 & 4.3335309 & 99.5 & .28414127 & 4.3221599 \\
\hline 94.6 & .26293403 & 4.3333381 & 99.6 & .28457348 & 4.3219020 \\
\hline 94.7 & .26336735 & 4.3331436 & 99.7 & .28500565 & 4.3216432 \\
\hline 94.8 & .26380066 & 4.3329476 & 99.8 & .28543780 & 4.3213833 \\
\hline 94.9 & .26423394 & 4.3327499 & 99.9 & .28586993 & 4.3211225 \\
\hline 95.0 & .26466721 & 4.3325507 & 100.0 & .28630203 & 4.3208607 \\
\hline
\end{tabular}


IPTS-68 Reference Function and Its Derivative With the Argument in Kelvin-Continued

\begin{tabular}{|c|c|c|c|c|c|}
\hline $\begin{array}{l}T \\
\mathbf{K}\end{array}$ & $W^{*}(T)$ & $\begin{array}{c}d W^{*}(T) / d T \\
\times 1000 \\
\mathrm{~K}^{-1}\end{array}$ & $\begin{array}{l}T \\
\mathbf{K}\end{array}$ & $W^{*}(T)$ & $\begin{array}{c}d W^{*}(T) / d T \\
\times 1000 \\
\mathbf{K}^{-1}\end{array}$ \\
\hline 100.0 & .28630203 & 4.3208607 & 150.0 & 49861134 & 4.1769430 \\
\hline 101.0 & .29062156 & 4.3181928 & 151.0 & 50278711 & 4.1746153 \\
\hline 102.0 & .29493839 & 4.3154407 & 152.0 & 50696058 & 4.1723135 \\
\hline 103.0 & 29925242 & 4.3126140 & 153.0 & 51113175 & 4.1700371 \\
\hline 104.0 & .30356359 & 4.3097216 & 154.0 & 51530066 & 4.1677859 \\
\hline 105.0 & .30787184 & 4.3067718 & 155.0 & 51946733 & 4.1655591 \\
\hline 106.0 & .31217712 & 4.3037723 & 156.0 & .52363178 & 4.1633567 \\
\hline 107.0 & .31647937 & 4.3007301 & 157.0 & 52779405 & 4.1611778 \\
\hline 108.0 & .32077857 & 4.2976521 & 158.0 & .53195415 & 4.1590222 \\
\hline 109.0 & .32507467 & 4.2945442 & 159.0 & 53611210 & 4.1568895 \\
\hline 110.0 & .32936765 & 4.2914122 & 160.0 & .54026793 & 4.1547790 \\
\hline 111.0 & .33365749 & 4.2882612 & 161.0 & 54442167 & 4.1526903 \\
\hline 112.0 & .33794417 & 4.2850962 & 162.0 & 54857332 & 4.1506230 \\
\hline 113.0 & .34222768 & 4.2819217 & 163.0 & .55272292 & 4.1485767 \\
\hline 114.0 & .34650801 & 4.2787417 & 164.0 & .55687048 & 4.1465508 \\
\hline 115.0 & .35078516 & 4.2755600 & 165.0 & .56101603 & 4.1445449 \\
\hline 116.0 & .35505913 & 4.2723801 & 166.0 & .56515958 & 4.1425586 \\
\hline 117.0 & .35932992 & 4.2692052 & 167.0 & .56930115 & 4.1405914 \\
\hline 118.0 & .36359754 & 4.2660381 & 168.0 & .57344077 & 4.1386427 \\
\hline 119.0 & .36786200 & 4.2628816 & 169.0 & .57757844 & 4.1367123 \\
\hline 120.0 & .37212331 & 4.2597380 & 170.0 & .58171420 & 4.1347998 \\
\hline 121.0 & .37638148 & 4.2566095 & 171.0 & .58584805 & 4.1329045 \\
\hline 122.0 & .38063653 & 4.2534980 & 172.0 & 58998001 & 4.1310262 \\
\hline 123.0 & .38488848 & 4.2504055 & 173.0 & 59411011 & 4.1291644 \\
\hline 124.0 & .38913735 & 4.2473335 & 174.0 & .59823835 & 4.1273188 \\
\hline 125.0 & .39338316 & 4.2442834 & 175.0 & .60236475 & 4.1254889 \\
\hline 126.0 & .39762593 & 4.2412563 & 176.0 & .60648933 & 4.1236743 \\
\hline 127.0 & .40186568 & 4.2382537 & 177.0 & 61061210 & 4.1218746 \\
\hline 128.0 & .40610244 & 4.2352763 & 178.0 & .61473308 & 4.1200896 \\
\hline 129.0 & .41033624 & 4.2323250 & 179.0 & .61885229 & 4.1183188 \\
\hline 130.0 & .41456710 & 4.2294006 & 180.0 & .62296973 & 4.1165619 \\
\hline 131.0 & .41879505 & 4.2265037 & 181.0 & .62708542 & 4.1148185 \\
\hline 132.0 & .42302012 & 4.2236348 & 182.0 & 63119937 & 4.1130884 \\
\hline 133.0 & .42724233 & 4.2207945 & 183.0 & .63531160 & 4.1113711 \\
\hline 134.0 & .43146172 & 4.2179829 & 184.0 & .63942211 & 4.1096663 \\
\hline 135.0 & .43567831 & 4.2152003 & 185.0 & .64353093 & 4.1079738 \\
\hline 136.0 & .43989213 & 4.2124470 & 186.0 & .64763807 & 4.1062933 \\
\hline 137.0 & .44410321 & 4.2097231 & 187.0 & .65174352 & 4.1046244 \\
\hline 138.0 & .44831158 & 4.2070286 & 188.0 & .65584732 & 4.1029669 \\
\hline 139.0 & .45251728 & 4.2043635 & 189.0 & .65994946 & 4.1013205 \\
\hline 140.0 & .45672032 & 4.2017278 & 190.0 & .66404996 & 4.0996849 \\
\hline 141.0 & .46092074 & 4.1991213 & 191.0 & .66814884 & 4.0980600 \\
\hline 142.0 & .46511857 & 4.1965439 & 192.0 & .67224609 & 4.0964453 \\
\hline 143.0 & .46931384 & 4.1939955 & 193.0 & .67634173 & 4.0948408 \\
\hline 144.0 & .47350657 & 4.1914757 & 194.0 & .68043577 & 4.0932461 \\
\hline 145.0 & .47769680 & 4.1889844 & 195.0 & .68452823 & 4.0916611 \\
\hline 146.0 & .48188455 & 4.1865212 & 196.0 & .68861910 & 4.0900855 \\
\hline 147.0 & .48606985 & 4.1840858 & 197.0 & .69270840 & 4.0885191 \\
\hline 148.0 & .49025273 & 4.1816779 & 198.0 & .69679614 & 4.0869618 \\
\hline 149.0 & .49443322 & 4.1792971 & 199.0 & .70088233 & 4.0854132 \\
\hline 150.0 & .49861134 & 4.1769430 & 200.0 & .70496697 & 4.0838734 \\
\hline
\end{tabular}


PTS-68 Reference Function and Its Derivative With the Argument in Kelvin-Continued

\begin{tabular}{|c|c|c|c|c|c|}
\hline $\begin{array}{l}T \\
\mathrm{~K}\end{array}$ & $W^{*}(T)$ & $\begin{array}{c}d W^{*}(T) / d T \\
\times 1000 \\
K^{-1}\end{array}$ & $\begin{array}{l}\boldsymbol{T} \\
\mathrm{K}\end{array}$ & $W^{*}(T)$ & $\begin{array}{c}d W^{*}(T) / d T \\
\times 1000 \\
\mathrm{~K}^{-1}\end{array}$ \\
\hline 200.0 & .70496697 & 4.0838734 & 250.0 & .90738312 & 4.0150756 \\
\hline 201.0 & .70905008 & 4.0823419 & 251.0 & .91139757 & 4.0138199 \\
\hline 202.0 & .71313166 & 4.0808187 & 252.0 & .91541076 & 4.0125672 \\
\hline 203.0 & .71721172 & 4.0793037 & 253.0 & .91942270 & 4.0113173 \\
\hline 204.0 & .72129027 & 4.0777966 & 254.0 & 92343340 & 4.0100702 \\
\hline 205.0 & .72536731 & 4.0762973 & 255.0 & .92744285 & 4.0088257 \\
\hline 206.0 & .72944286 & 4.0748056 & 256.0 & .93145105 & 4.0075837 \\
\hline 207.0 & .73351693 & 4.0733215 & 257.0 & .93545801 & 4.0063439 \\
\hline 208.0 & .73758951 & 4.0718447 & 258.0 & .93946374 & 4.0051063 \\
\hline 209.0 & .74166062 & 4.0703751 & 259.0 & 94346823 & 4.0038708 \\
\hline 210.0 & .74573026 & 4.0689126 & 260.0 & 94747148 & 4.0026371 \\
\hline 211.0 & .74979844 & 4.0674572 & 261.0 & .95147350 & 4.0014051 \\
\hline 212.0 & .75386518 & 4.0660085 & 262.0 & .95547429 & 4.0001747 \\
\hline 213.0 & .75793046 & 4.0645666 & 263.0 & .95947385 & 3.9989456 \\
\hline 214.0 & .76199431 & 4.0631313 & 264.0 & .96347218 & 3.9977178 \\
\hline 215.0 & .76605673 & 4.0617025 & 265.0 & .96746929 & 3.9964910 \\
\hline 216.0 & .77011772 & 4.0602801 & 266.0 & .97146517 & 3.9952650 \\
\hline 217.0 & .77417729 & 4.0588640 & 267.0 & 97545982 & 3. 9940397 \\
\hline 218.0 & .77823545 & 4.0574541 & 268.0 & .97945325 & 3. 9928147 \\
\hline 219.0 & .78229220 & 4.0560503 & 269.0 & 98344545 & 3. 9915902 \\
\hline 220.0 & .78634755 & 4.0546525 & 270.0 & 98743643 & 3.9903656 \\
\hline 221.0 & .79040151 & 4.0532606 & 271.0 & 99142618 & 3. 9891409 \\
\hline 222.0 & .79445408 & 4.0518746 & 272.0 & 99541471 & 3.9879159 \\
\hline 223.0 & .79850526 & 4.0504942 & 273.0 & 99940201 & 3. 9866902 \\
\hline 224.0 & .80255507 & 4.0491195 & 273.15 & 1.00000000 & 3.9865063 \\
\hline 225.0 & .80660350 & 4.0477504 & & & \\
\hline 226.0 & .81065057 & 4.0463867 & & & \\
\hline 227.0 & .81469628 & 4.0450285 & & & \\
\hline 228.0 & .81874063 & 4.0436755 & & & \\
\hline 229.0 & .82278363 & 4.0423277 & & & \\
\hline 230.0 & .82682528 & 4.0409851 & & & \\
\hline 231.0 & .83086560 & 4.0396475 & & & \\
\hline 232.0 & .83490458 & 4.0383149 & & & \\
\hline 233.0 & .83894223 & 4.0369871 & & & \\
\hline 234.0 & .84297856 & 4.0356641 & & & \\
\hline 235.0 & .84701356 & 4.0343459 & & & \\
\hline 236.0 & .85104725 & 4.0330323 & & & \\
\hline 237.0 & .85507963 & 4.0317232 & & & \\
\hline 238.0 & .85911070 & 4.0304186 & & & \\
\hline 239.0 & .86314047 & 4.0291183 & & & \\
\hline 240.0 & .86716894 & 4.0278223 & & & \\
\hline 241.0 & .87119611 & 4.0265305 & & & \\
\hline 242.0 & .87522200 & 4.0252428 & & & \\
\hline 243.0 & .87924660 & 4.0239590 & & & \\
\hline 244.0 & .88326992 & 4.0226792 & & & \\
\hline 245.0 & 88729196 & 4.0214031 & & & \\
\hline 246.0 & .89131273 & 4.0201307 & & & \\
\hline 247.0 & .89533222 & 4.0188618 & & & \\
\hline 248.0 & 89935045 & 4.0175964 & & & \\
\hline 249.0 & 90336742 & 40163344 & & & \\
\hline 250.0 & .90738312 & 4.0150756 & & & \\
\hline
\end{tabular}


IPTS-68 Reference Function and Its First Derivative With the Argument in Celsius

\begin{tabular}{|c|c|c|c|c|c|}
\hline$\stackrel{t}{{ }^{t} \mathrm{C}}$ & $W^{*}(t)$ & $\begin{array}{c}d W^{*}(t) / d T \\
\times 1000 \\
{ }^{\circ} \mathrm{C}^{-1}\end{array}$ & ${ }^{t} \mathrm{C}$ & $W^{*}(t)$ & $\begin{array}{c}d W^{*}(t) / d T \\
\times 1000 \\
{ }^{\circ} \mathrm{C}^{-1}\end{array}$ \\
\hline-260.0 & 00126168 & .2107490 & -255.0 & .00309642 & 5540898 \\
\hline-259.9 & 00128300 & .2158042 & -254.9 & .00315227 & 5628522 \\
\hline-259.8 & 00130484 & .2209403 & -254.8 & .00320900 & 5716830 \\
\hline-259.7 & .00132719 & .2261558 & -254.7 & .00326661 & 5805814 \\
\hline-259.6 & 00135007 & .2314492 & -254.6 & 00332511 & .5895467 \\
\hline-259.5 & .00137349 & .2368191 & -254.5 & .00338452 & 5985782 \\
\hline-259.4 & .00139744 & .2422640 & -254.4 & .00344483 & 6076751 \\
\hline-259.3 & .00142194 & .2477828 & -254.3 & .00350606 & 6168368 \\
\hline-259.2 & .00144700 & .2533743 & -254.2 & .00356820 & 6260627 \\
\hline-259.1 & .00147262 & .2590375 & -254.1 & .00363127 & 6353522 \\
\hline-259.0 & .00149881 & .2647716 & -254.0 & .00369527 & 6447048 \\
\hline-258.9 & .00152557 & .2705758 & -253.9 & .00376022 & 6541199 \\
\hline-258.8 & .00155293 & .2764497 & -253.8 & .00382610 & 6635970 \\
\hline-258.7 & .00158087 & .2823930 & -253.7 & .00389294 & 6731357 \\
\hline-258.6 & .00160941 & .2884054 & -253.6 & .00396073 & .6827354 \\
\hline-258.5 & .00163855 & .2944869 & -253.5 & .00402949 & 6923958 \\
\hline-258.4 & .00166831 & .3006377 & -253.4 & .00409921 & 7021165 \\
\hline-258.3 & .00169868 & 3068581 & -253.3 & .00416991 & 7118970 \\
\hline-258.2 & .00172968 & .3131483 & -253.2 & .00424159 & .7217370 \\
\hline-258.1 & .00176131 & 3195089 & -253.1 & .00431426 & 7316359 \\
\hline-258.0 & .00179358 & .3259405 & -253.0 & .00438792 & .7415936 \\
\hline-257.9 & .00182650 & .3324437 & -252.9 & .00446258 & 7516094 \\
\hline-257.8 & .00186007 & .3390193 & -252.8 & .00453825 & 7616831 \\
\hline-257.7 & .00189431 & .3456678 & -252.7 & .00461492 & 7718143 \\
\hline-257.6 & .00192921 & .3523901 & -252.6 & .00469261 & 7820023 \\
\hline-257.5 & .00196479 & .3591870 & -252.5 & .00477132 & 7922469 \\
\hline-257.4 & .00200105 & .3660590 & -252.4 & .00485106 & 8025475 \\
\hline-257.3 & .00203800 & .3730068 & -252.3 & .00493183 & .8129036 \\
\hline-257.2 & .00207565 & .3800312 & -252.2 & .00501364 & .8233146 \\
\hline-257.1 & .00211401 & 3871326 & -252.1 & .00509650 & 8337801 \\
\hline-257.0 & .00215308 & .3943114 & -252.0 & .00518040 & 8442993 \\
\hline-256.9 & .00219288 & .4015681 & -251.9 & .00526536 & 8548717 \\
\hline-256.8 & .00223340 & .4089030 & -251.8 & .00535138 & 8654965 \\
\hline-256.7 & .00227466 & .4163162 & -251.7 & .00543846 & 8761731 \\
\hline-256.6 & .00231667 & .4238079 & -251.6 & .00552661 & 8869007 \\
\hline-256.5 & .00235942 & .4313780 & -251.5 & .00561584 & 8976786 \\
\hline-256.4 & .00240294 & .4390265 & -251.4 & .00570615 & 9085058 \\
\hline-256.3 & .00244723 & 4467532 & -251.3 & .00579755 & 9193817 \\
\hline-256.2 & .00249230 & 4545578 & -251.2 & .00589003 & 9303052 \\
\hline-256.1 & .00253815 & 4624399 & -251.1 & .00598361 & 9412756 \\
\hline-256.0 & .00258479 & 4703991 & -251.0 & .00607829 & 9522917 \\
\hline-255.9 & .00263223 & .4784348 & -250.9 & .00617407 & 9633528 \\
\hline-255.8 & .00268048 & 4865465 & -250.8 & 00627096 & 9744577 \\
\hline-255.7 & .00272954 & 4947336 & -250.7 & .00636896 & 9856055 \\
\hline-255.6 & .00277943 & 5029952 & -250.6 & .00646808 & 9967951 \\
\hline-255.5 & .00283014 & 5113308 & -250.5 & .00656832 & 1.0080255 \\
\hline-255.4 & .00288170 & .5197395 & -250.4 & .00666969 & 1.0192956 \\
\hline-255.3 & .00293409 & .5282205 & -250.3 & .00677218 & 1.0306044 \\
\hline-255.2 & .00298734 & .5367731 & -250.2 & .00687581 & 1.0419507 \\
\hline-255.1 & 00304145 & .5453965 & -250.1 & .00698057 & 1.0533335 \\
\hline-255.0 & 00309642 & .5540898 & -250.0 & .00708648 & 1.0647516 \\
\hline
\end{tabular}


IPTS-68 Reference Function and Its First Derivative With the Argument in Celsius-Continued

\begin{tabular}{|c|c|c|c|c|c|}
\hline$\stackrel{t}{{ }^{\circ} \mathrm{C}}$ & $W^{*}(t)$ & $\begin{array}{c}d W^{*}(t) / d T \\
\times 1000 \\
{ }^{\circ} \mathrm{C}^{-1}\end{array}$ & $\stackrel{t}{{ }^{t} \mathrm{C}}$ & $W^{*}(t)$ & $\begin{array}{c}d W^{*}(t) / d T \\
\times 1000 \\
{ }^{\circ} \mathrm{C}^{-1}\end{array}$ \\
\hline-250.0 & .00708648 & 1.0647516 & -245.0 & .01388681 & 1.6610569 \\
\hline-249.9 & .00719352 & 1.0762039 & -244.9 & .01405353 & 1.6732110 \\
\hline-249.8 & .00730172 & 1.0876895 & -244.8 & .01422146 & 1.6853661 \\
\hline-249.7 & .00741106 & 1.0992071 & -244.7 & 01439060 & 1.6975216 \\
\hline-249.6 & .00752156 & 1.1107558 & -244.6 & 01456096 & 1.7096772 \\
\hline-249.5 & .00763322 & 1.1223343 & -244.5 & 01473254 & 1.7218324 \\
\hline-249.4 & .00774603 & 1.1339418 & -244.4 & .01490533 & 1.7339866 \\
\hline-249.3 & .00786000 & 1. 1455771 & -244.3 & .01507933 & 1.7461396 \\
\hline-249.2 & .00797515 & 1.1572392 & -244.2 & .01525455 & 1.7582907 \\
\hline-249.1 & .00809145 & 1.1689271 & -244.1 & .01543099 & 1.7704395 \\
\hline-249.0 & .00820893 & 1.1806398 & -244.0 & .01560864 & 1.7825855 \\
\hline-248.9 & .00832758 & 1.1923763 & -243.9 & .01578751 & 1.7947282 \\
\hline-248.8 & .00844741 & 1.2041358 & -243.8 & .01596759 & 1.8068671 \\
\hline-248.7 & .00856841 & 1.2159172 & -243.7 & .01614888 & 1.8190017 \\
\hline-248.6 & .00869059 & 1.2277198 & -243.6 & .01633139 & 1.8311315 \\
\hline-248.5 & .00881395 & 1.2395426 & -243.5 & .01651511 & 1.8432558 \\
\hline-248.4 & .00893850 & 1.2513847 & -243.4 & .01670004 & 1.8553743 \\
\hline-248.3 & .00906423 & 1.2632455 & -243.3 & .01688618 & 1.8674863 \\
\hline-248.2 & .00919115 & 1.2751240 & -243.2 & .01707354 & 1.8795912 \\
\hline-248.1 & .00931926 & 1.2870195 & -243.1 & .01726210 & 1.8916886 \\
\hline-248.0 & .00944856 & 1.2989314 & -243.0 & .01745187 & 1.9037778 \\
\hline-247.9 & .00957904 & 1.3108588 & -242.9 & .01764286 & 1.9158583 \\
\hline-247.8 & .00971073 & 1.3228011 & -242.8 & .01783504 & 1.9279295 \\
\hline-247.7 & .00984361 & 1.3347576 & -242.7 & .01802844 & 1.9399908 \\
\hline-247.6 & .00997768 & 1.3467277 & -242.6 & .01822304 & 1.9520417 \\
\hline-247.5 & .01011295 & 1.3587108 & -242.5 & 01841885 & 1.9640815 \\
\hline-247.4 & .01024942 & 1.3707063 & -242.4 & .01861586 & 1.9761096 \\
\hline-247.3 & .01038709 & 1.3827136 & -242.3 & 01881407 & 1.9881255 \\
\hline-247.2 & .01052597 & 1.3947322 & -242.2 & .01901348 & 2.0001285 \\
\hline-247.1 & .01066604 & 1.4067616 & -242.1 & .01921410 & 2.0121180 \\
\hline-247.0 & .01080732 & 1.4188011 & -242.0 & .01941591 & 2.0240935 \\
\hline-246.9 & .01094980 & 1.4308504 & -241.9 & .01961891 & 2.0360544 \\
\hline-246.8 & .01109349 & 1.4429090 & -241.8 & .01982312 & 2.0480000 \\
\hline-246.7 & .01123838 & 1.4549764 & -241.7 & .02002851 & 2.0599297 \\
\hline-246.6 & .01138448 & 1.4670521 & -241.6 & .02023510 & 2.0718429 \\
\hline-246.5 & .01153179 & 1.4791358 & -241.5 & .02044288 & 2.0837391 \\
\hline-246.4 & .01168031 & 1.4912269 & -241.4 & .02065185 & 2.0956176 \\
\hline-246.3 & .01183004 & 1.5033250 & -241.3 & .02086200 & 2.1074779 \\
\hline-246.2 & .01198098 & 1.5154297 & -241.2 & .02107334 & 2.1193193 \\
\hline-246.1 & .01213312 & 1.5275407 & -241.1 & .02128587 & 2.1311413 \\
\hline-246.0 & .01228648 & 1.5396575 & -241.0 & .02149957 & 2.1429433 \\
\hline-245.9 & .01244106 & 1.5517797 & -240.9 & .02171446 & 2.1547247 \\
\hline-245.8 & .01259684 & 1.5639069 & -240.8 & .02193052 & 2.1664849 \\
\hline-245.7 & .01275384 & 1.5760388 & -240.7 & .02214775 & 2.1782235 \\
\hline-245.6 & .01291205 & 1.5881748 & -240.6 & .02236616 & 2. 1899397 \\
\hline-245.5 & .01307147 & 1.6003146 & -240.5 & .02258574 & 2.2016331 \\
\hline-245.4 & .01323211 & 1.6124579 & -240.4 & .02280649 & 2.2133031 \\
\hline-245.3 & .01339396 & 1.6246042 & -240.3 & .02302840 & 2. 2249492 \\
\hline-245.2 & .01355703 & 1.6367530 & -240.2 & .02325147 & 2.2365708 \\
\hline-245.1 & .01372132 & 1.6489041 & -240.1 & .02347571 & 2.2481674 \\
\hline 45.0 & .01388681 & 1.6610569 & -240.0 & .02370111 & 2.2597386 \\
\hline
\end{tabular}


IPTS-68 Reference Function and Its First Derivative With the Argument in Celsius - Continued

\begin{tabular}{|c|c|c|c|c|c|}
\hline$\stackrel{t}{{ }^{\circ} \mathrm{C}}$ & $W^{*}(t)$ & $\begin{array}{c}d W^{*}(t) / d T \\
\times 1000 \\
{ }^{\circ} \mathrm{C}^{-1}\end{array}$ & $\stackrel{t}{{ }^{\circ} \mathrm{C}}$ & $W^{*}(t)$ & $\begin{array}{c}d W^{*}(t) / d T \\
\times 1000 \\
{ }^{\circ} \mathrm{C}^{-1}\end{array}$ \\
\hline-240.0 & .02370111 & 2.2597386 & -235.0 & .03638008 & 2.7974138 \\
\hline-239.9 & .02392766 & 2.2712837 & -234.9 & .03666031 & 2.8072404 \\
\hline-239.8 & .02415536 & 2.2828024 & -234.8 & .03694152 & 2.8170283 \\
\hline-239.7 & .02438422 & 2. 2942942 & -234.7 & .03722371 & 2.8267775 \\
\hline-239.6 & .02461422 & 2.3057584 & -234.6 & .03750688 & 2.8364880 \\
\hline-239.5 & .02484537 & 2.3171947 & -234.5 & .03779101 & 2.8461597 \\
\hline-239.4 & .02507766 & 2.3286027 & -234.4 & .03807611 & 2.8557927 \\
\hline-239.3 & .02531109 & 2.3399819 & -234.3 & .03836217 & 2.8653868 \\
\hline-239.2 & .02554565 & 2.3513318 & -234.2 & .03864919 & 2.8749421 \\
\hline-239.1 & .02578135 & 2.3626520 & -234.1 & .03893716 & 2.8844585 \\
\hline-239.0 & .02601818 & 2.3739421 & -234.0 & .03922608 & 2.8939361 \\
\hline-238.9 & .02625614 & 2.3852018 & -233.9 & .03951594 & 2.9033747 \\
\hline-238.8 & .02649522 & 2.3964305 & -233.8 & .03980675 & 2.9127745 \\
\hline-238.7 & .02673543 & 2.4076280 & -233.7 & .04009850 & 2.9221354 \\
\hline-238.6 & .02697675 & 2.4187939 & -233.6 & .04039118 & 2.9314574 \\
\hline-238.5 & .02721918 & 2.4299277 & -233.5 & .04068479 & 2.9407404 \\
\hline-238.4 & .02746273 & 2.4410292 & -233.4 & .04097932 & 2.9499846 \\
\hline-238.3 & .02770739 & 2.4520979 & -233.3 & .04127478 & 2.9591898 \\
\hline-238.2 & .02795315 & 2.4631337 & -233.2 & 04157116 & 2.9683562 \\
\hline-238.1 & .02820001 & 2.4741362 & -233.1 & 04186845 & 2.9774837 \\
\hline-238.0 & .02844798 & 2.4851050 & -233.0 & 04216665 & 2.9865723 \\
\hline-237.9 & .02869703 & 2.4960398 & -232.9 & 04246576 & 2.9956221 \\
\hline-237.8 & .02894718 & 2.5069405 & -232.8 & 04276578 & 3.0046329 \\
\hline-237.7 & .02919842 & 2.5178066 & -232.7 & 04306669 & 3.0136050 \\
\hline-237.6 & .02945074 & 2.5286380 & -232.6 & 04336850 & 3.0225383 \\
\hline-237.5 & .02970415 & 2.5394344 & -232.5 & 04367120 & 3.0314327 \\
\hline-237.4 & .02995863 & 2.5501956 & -232.4 & .04397478 & 3.0402884 \\
\hline-237.3 & .03021419 & 2.5609212 & -232.3 & .04427925 & 3.0491053 \\
\hline-237.2 & .03047081 & 2.5716111 & -232.2 & .04458460 & 3.0578836 \\
\hline-237.1 & .03072851 & 2.5822652 & -232.1 & .04489083 & 3.0666231 \\
\hline-237.0 & .03098726 & 2.5928830 & -232.0 & 04519793 & 3.0753239 \\
\hline-236.9 & .03124708 & 2.6034646 & -231.9 & 04550589 & 3.0839861 \\
\hline-236.8 & .03150796 & 2.6140096 & -231.8 & 04581472 & 3.0926096 \\
\hline-236.7 & .03176988 & 2.6245180 & -231.7 & .04612441 & 3.1011946 \\
\hline-236.6 & .03203286 & 2.6349895 & -231.6 & .04643496 & 3.1097409 \\
\hline-236.5 & .03229688 & 2.6454240 & -231.5 & .04674636 & 3.1182488 \\
\hline-236.4 & .03256194 & 2.6558212 & -231.4 & .04705861 & 3.1267181 \\
\hline-236.3 & .03282804 & 2.6661812 & -231.3 & 04737170 & 3.1351490 \\
\hline-236.2 & .03309518 & 2.6765038 & -231.2 & .04768564 & 3.1435415 \\
\hline-236.1 & 03336334 & 2.6867888 & -231.1 & .04800041 & 3.1518955 \\
\hline-236.0 & 03363253 & 2.6970360 & -231.0 & .04831601 & 3.1602111 \\
\hline-235.9 & 03390275 & 2.7072455 & -230.9 & 04863245 & 3.1684884 \\
\hline-235.8 & .03417398 & 2.7174171 & -230.8 & .04894971 & 3.1767274 \\
\hline-235.7 & .03444623 & 2.7275506 & -230.7 & 04926779 & 3. 1849282 \\
\hline-235.6 & .03471949 & 2.7376460 & -230.6 & 04958669 & 3. 1930907 \\
\hline-235.5 & .03499376 & 2.7477032 & -230.5 & 04990641 & 3.2012150 \\
\hline-235.4 & .03526903 & 2.7577222 & -230.4 & 05022694 & 3.2093011 \\
\hline-235.3 & .03554530 & 2.7677028 & -230.3 & .05054827 & 3.2173491 \\
\hline-235.2 & .03582257 & 2.7776450 & -230.2 & .05087040 & 3.2253591 \\
\hline-235.1 & .03610083 & 2.7875486 & -230.1 & .05119334 & 3.2333310 \\
\hline-235.0 & .03638008 & 2.7974138 & -230.0 & .05151707 & 3.2412648 \\
\hline
\end{tabular}


IPTS-68 Reference Function and Its First Derivative With the Argument in Celsius - Continued

\begin{tabular}{|c|c|c|c|c|c|}
\hline${ }^{t} \mathrm{C}$ & $W^{*}(t)$ & $\begin{array}{c}d W^{*}(t) / d T \\
\times 1000 \\
{ }^{\circ} \mathrm{C}^{-1}\end{array}$ & $\stackrel{t}{{ }^{t} \mathrm{C}}$ & $W^{*}(t)$ & $\begin{array}{c}d W^{*}(t) / d T \\
\times 1000 \\
{ }^{\circ} \mathrm{C}^{-1}\end{array}$ \\
\hline-230.0 & .05151707 & 3.2412648 & -225.0 & 06863481 & 3.5905076 \\
\hline-229.9 & 05184159 & 3.2491608 & -224.9 & .06899417 & 3.5965674 \\
\hline-229.8 & 05216690 & 3.2570187 & -224.8 & .06935412 & 3.6025922 \\
\hline-229.7 & 05249299 & 3.2648389 & -224.7 & 06971468 & 3.6085818 \\
\hline-229.6 & .05281987 & 3.2726211 & -224.6 & 07007584 & 3.6145364 \\
\hline-229.5 & .05314752 & 3.2803655 & -224.5 & 07043759 & 3.6204562 \\
\hline-229.4 & 05347594 & 3.2880722 & -224.4 & 07079993 & 3.6263411 \\
\hline-229.3 & .05380513 & 3.2957411 & -224.3 & 07116286 & 3.6321912 \\
\hline-229.2 & .05413509 & 3.3033724 & -224.2 & 07152637 & 3.6380067 \\
\hline-229.1 & .05446580 & 3.3109660 & -224.1 & 07189046 & 3.6437876 \\
\hline-229.0 & .05479728 & 3.3185221 & -224.0 & 07225512 & 3.6495340 \\
\hline-228.9 & .05512951 & 3.3260405 & -223.9 & .07262036 & 3.6552461 \\
\hline-228.8 & .05546248 & 3.3335215 & -223.8 & .07298617 & 3.6609237 \\
\hline-228.7 & .05579621 & 3.3409650 & -223.7 & .07335255 & 3.6665672 \\
\hline-228.6 & .05613068 & 3.3483711 & -223.6 & .07371948 & 3.6721765 \\
\hline-228.5 & .05646588 & 3.3557398 & -223.5 & .07408698 & 3.6777517 \\
\hline-228.4 & .05680182 & 3.3630712 & -223.4 & .07445503 & 3.6832930 \\
\hline-228.3 & .05713850 & 3.3703654 & -223.3 & .07482364 & 3.6888004 \\
\hline-228.2 & .05747589 & 3.3776222 & -223.2 & .07519279 & 3.6942740 \\
\hline-228.1 & .05781402 & 3.3848420 & -223.1 & .07556249 & 3.6997139 \\
\hline-228.0 & .05815286 & 3. 3920245 & -223.0 & .07593273 & 3.7051203 \\
\hline-227.9 & .05849242 & 3.3991700 & -222.9 & .07630351 & 3.7104931 \\
\hline-227.8 & .05883269 & 3.4062785 & -222.8 & .07667483 & 3.7158326 \\
\hline-227.7 & .05917368 & 3.4133499 & -222.7 & 07704668 & 3.7211387 \\
\hline-227.6 & .05951536 & 3.4203845 & -222.6 & 07741906 & 3.7264117 \\
\hline-227.5 & .05985775 & 3.4273822 & -222.5 & .07779196 & 3.7316515 \\
\hline-227.4 & .06020084 & 3.4343430 & -222.4 & .07816539 & 3.7368584 \\
\hline-227.3 & .06054462 & 3.4412671 & -222.3 & .07853933 & 3.7420323 \\
\hline-227.2 & .06088909 & 3.4481544 & -222.2 & .07891379 & 3.7471734 \\
\hline-227.1 & .06123425 & 3.4550051 & -222.1 & .07928877 & 3.7522819 \\
\hline-227.0 & .06158009 & 3.4618192 & -222.0 & .07966425 & 3.7573578 \\
\hline-226.9 & .06192661 & 3.4685967 & -221.9 & .08004024 & 3.7624011 \\
\hline-226.8 & .06227381 & 3.4753378 & -221.8 & .08041673 & 3.7674122 \\
\hline-226.7 & .06262168 & 3.4820424 & -221.7 & 08079372 & 3.7723909 \\
\hline-226.6 & .06297022 & 3.4887106 & -221.6 & .08117120 & 3.7773375 \\
\hline-226.5 & .06331942 & 3.4953426 & -221.5 & .08154918 & 3.7822520 \\
\hline-226.4 & .06366928 & 3.5019382 & -221.4 & .08192765 & 3.7871346 \\
\hline-226.3 & .06401981 & 3.5084977 & -221.3 & .08230661 & 3.7919854 \\
\hline-226.2 & .06437098 & 3.5150211 & -221.2 & .08268605 & 3.7968044 \\
\hline-226.1 & .06472281 & 3.5215084 & -221.1 & .08306597 & 3.8015918 \\
\hline-226.0 & .06507528 & 3.5279597 & -221.0 & .08344637 & 3.8063478 \\
\hline-225.9 & .06542840 & 3.5343750 & -220.9 & .08382724 & 3.8110723 \\
\hline-225.8 & .06578216 & 3.5407545 & -220.8 & .08420858 & 3.8157656 \\
\hline-225.7 & .06613655 & 3.5470982 & -220.7 & .08459039 & 3.8204278 \\
\hline-225.6 & .06649157 & 3.5534062 & -220.6 & .08497266 & 3.8250588 \\
\hline-225.5 & .06684723 & 3.5596785 & -220.5 & .08535540 & 3.8296591 \\
\hline-225.4 & .06720351 & 3.5659152 & -220.4 & .08573860 & 3.8342284 \\
\hline-225.3 & .06756041 & 3.5721164 & -220.3 & 08612225 & 3.8387671 \\
\hline-225.2 & .06791793 & 3.5782821 & -220.2 & .08650635 & 3.8432753 \\
\hline-225.1 & .06827607 & 3.5844125 & -220.1 & 08689090 & 3.8477530 \\
\hline-225.0 & .06863481 & 3.5905076 & -220.0 & .08727590 & 3.8522003 \\
\hline
\end{tabular}


IPTS-68 Reference Function and Its First Derivative With the Argument in Celsius - Continued

\begin{tabular}{|c|c|c|c|c|c|}
\hline${ }^{t} \mathrm{C}$ & $W^{*}(t)$ & $\begin{array}{c}d W^{*}(t) / d T \\
\times 1000 \\
{ }^{\circ} \mathrm{C}^{-1}\end{array}$ & $\stackrel{\circ}{ }^{t} \mathrm{C}$ & $W^{*}(t)$ & $\begin{array}{c}d W^{*}(t) / d T \\
\times 1000 \\
{ }^{\circ} \mathrm{C}^{-1}\end{array}$ \\
\hline-220.0 & .08727590 & 3.8522003 & -215.0 & .10703128 & 4.03869 \\
\hline-219.9 & .08766134 & 3.8566175 & -214.9 & .10743530 & 4.04176 \\
\hline-219.8 & .08804722 & 3.8610046 & -214.8 & .10783963 & $4.04480 €$ \\
\hline-219.7 & .08843354 & 3.8653617 & -214.7 & 10824426 & 4.047828 \\
\hline-219.6 & .08882029 & 3.8696890 & -214.6 & .10864919 & 4.050828 \\
\hline-219.5 & .08920748 & 3.8739866 & -214.5 & .10905443 & 4.053803 \\
\hline-219.4 & .08959509 & 3.8782546 & -214.4 & 10945995 & 4.056756 \\
\hline-219.3 & .08998313 & 3.8824931 & -214.3 & 10986578 & 4.059686 \\
\hline-219.2 & .09037159 & 3.8867023 & -214.2 & .11027189 & 4.062593 \\
\hline-219.1 & .09076046 & 3.8908823 & -214.1 & .11067829 & 4.065477 \\
\hline-219.0 & .09114976 & 3.8950332 & -214.0 & .11108498 & 4.068339 \\
\hline-218.9 & .09153947 & 3.8991550 & -213.9 & .11149196 & 4.071178 \\
\hline-218.8 & .09192959 & 3.9032481 & -213.8 & 11189922 & 4.073994 \\
\hline-218.7 & .09232012 & 3.9073125 & -213.7 & .11230676 & 4.076789 \\
\hline-218.6 & .09271105 & 3.9113482 & -213.6 & .11271458 & 4.079562 \\
\hline-218.5 & .09310239 & 3.9153555 & -213.5 & .11312267 & 4.082312 \\
\hline-218.4 & .09349412 & 3.9193345 & -213.4 & .11353104 & 4.085041 \\
\hline-218.3 & .09388625 & 3.9232852 & -213.3 & .11393968 & 4.087748 \\
\hline-218.2 & .09427878 & 3.9272080 & -213.2 & .11434859 & 4.090434 \\
\hline-218.1 & .09467169 & 3.9311027 & -213.1 & 11475776 & 4.093098 \\
\hline-218.0 & .09506500 & 3.9349697 & -213.0 & 11516721 & 4.095741 \\
\hline-217.9 & .09545869 & 39388089 & -212.9 & .11557691 & 4.098362 \\
\hline-217.8 & .09585276 & 3.9426206 & -212.8 & .11598688 & 4.100963 \\
\hline-217.7 & .09624721 & 3.9464048 & -212.7 & .11639710 & $4.103542^{\prime}$ \\
\hline-217.6 & .09664204 & 3.9501618 & -212.6 & .11680759 & 4.106101 \\
\hline-217.5 & .09703724 & 3.9538916 & -212.5 & 11721832 & 4.108639 \\
\hline-217.4 & .09743282 & 3.9575943 & -212.4 & 11762931 & 4.111157 \\
\hline-217.3 & .09782876 & 3.9612702 & -212.3 & 11804055 & 4.113654 \\
\hline-217.2 & .09822507 & 3.9649193 & -212.2 & 11845204 & 4.116130 \\
\hline-217.1 & .09862174 & 3.9685417 & -212.1 & 11886378 & 4.118587 \\
\hline-217.0 & .09901878 & 3.9721376 & -212.0 & 11927576 & 4.1210238 \\
\hline-216.9 & .09941617 & 3.9757072 & -211.9 & 11968798 & 4.123440 \\
\hline-216.8 & .09981392 & 3.9792505 & -211.8 & .12010045 & 4.125837 \\
\hline-216.7 & .10021202 & 3.9827677 & -211.7 & .12051315 & 4.1282140 \\
\hline-216.6 & .10061047 & 3.9862589 & -211.6 & .12092609 & 4.130571 \\
\hline-216.5 & .10100927 & 3.9897242 & -211.5 & .12133926 & 4.1329093 \\
\hline-216.4 & .10140841 & 3.9931638 & -211.4 & .12175267 & 4.1352278 \\
\hline-216.3 & .10180790 & 3.9965778 & -211.3 & .12216631 & 4.1375271 \\
\hline-216.2 & .10220773 & 3. 9999664 & -211.2 & 12258018 & 4.1398074 \\
\hline-216.1 & .10260789 & 4.0033296 & -211.1 & .12299427 & 4.1420686 \\
\hline-216.0 & .10300839 & 4.0066676 & -211.0 & .12340859 & 4.1443109 \\
\hline-215.9 & .10340923 & 4.0099806 & -210.9 & .12382313 & 4.1465345 \\
\hline-215.8 & .10381039 & 4.0132686 & -210.8 & .12423790 & 4.148739 \\
\hline-215.7 & .10421188 & 4.0165319 & -210.7 & 12465288 & 4.1509258 \\
\hline-215.6 & .10461370 & 4.0197704 & -210.6 & .12506808 & 4.1530938 \\
\hline-215.5 & .10501583 & 4.0229845 & -210.5 & 12548350 & 4.1552435 \\
\hline-215.4 & .10541829 & 4.0261741 & -210.4 & .12589913 & 4.1573750 \\
\hline-215.3 & .10582107 & 4.0293394 & -210.3 & .12631497 & 4.1594884 \\
\hline-215.2 & .10622416 & 4.0324806 & -210.2 & .12673103 & 4.1615839 \\
\hline-215.1 & .10662756 & 4.0355978 & -210.1 & 12714729 & 4.1636615 \\
\hline-215.0 & .10703128 & 4.0386911 & -210.0 & 12756376 & 4.1657214 \\
\hline
\end{tabular}


IPTS-68 Reference Function and Its First Derivative With the Argument in Celsius - Continued

\begin{tabular}{|c|c|c|c|c|c|}
\hline${ }^{t} \mathrm{C}$ & $W^{*}(t)$ & $\begin{array}{c}d W^{*}(t) / d T \\
\times \quad 1000 \\
{ }^{\circ} \mathrm{C}^{-1}\end{array}$ & 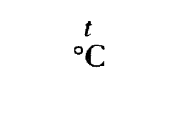 & $W^{*}(t)$ & $\begin{array}{c}d W^{*}(t) / d T \\
\times 1000 \\
{ }^{\circ} \mathrm{C}^{-1}\end{array}$ \\
\hline-210.0 & 12756376 & 4. 1657214 & -205.0 & .14861484 & 4.2484572 \\
\hline-209.9 & 12798043 & 4. 1677637 & -204.9 & .14903975 & 4.2497506 \\
\hline-209.8 & 12839731 & 4. 1697885 & -204.8 & .14946479 & 4.2510313 \\
\hline-209.7 & 12881439 & 4.1717959 & -204.7 & 14988995 & 4.2522996 \\
\hline-209.6 & 12923167 & 4.1737860 & -204.6 & .15031524 & 4.2535555 \\
\hline-209.5 & 12964914 & 4. 1757590 & -204.5 & .15074066 & 4.2547990 \\
\hline-209.4 & 13006682 & 4. 1777149 & -20.4 & .15116620 & 4.2560304 \\
\hline-209.3 & 13048469 & 4. 1796538 & -204.3 & .15159187 & 4.2572496 \\
\hline-209.2 & .13090275 & 4. 1815758 & -204.2 & .15201765 & 4.2584567 \\
\hline-209.1 & .13132100 & 4. 1834812 & -204.1 & .15244356 & 4.2596518 \\
\hline-209.0 & .13173944 & 4. 1853699 & -204.0 & .15286958 & 4.2608350 \\
\hline-208.9 & .13215808 & 4. 1872421 & -203.9 & .15329573 & 4.2620064 \\
\hline-208.8 & .13257689 & 4. 1890979 & -203.8 & .15372198 & 4.2631661 \\
\hline-208.7 & .13299589 & 4. 1909374 & -203.7 & .15414836 & 4.2643141 \\
\hline-208.6 & .13341508 & 4. 1927607 & -203.6 & .15457485 & 4.2654505 \\
\hline-208.5 & .13383445 & 4. 1945679 & -203.5 & .15500145 & 4.2665754 \\
\hline-208.4 & .13425399 & 4. 1963591 & -203.4 & .15542816 & 4.2676889 \\
\hline-208.3 & .13467372 & 4. 1981344 & -203.3 & .15585499 & 4.2687911 \\
\hline-208.2 & .13509362 & 4.1998939 & -203.2 & .15628192 & 4.2698819 \\
\hline-208.1 & .13551370 & 4.2016377 & -203.1 & .15670896 & 4.2709616 \\
\hline-208.0 & .13593395 & 4.2033660 & -203.0 & .15713611 & 4.2720302 \\
\hline-207.9 & .13635437 & 4.2050788 & -202.9 & 15756337 & 4. 2730877 \\
\hline-207.8 & .13677496 & 4.2067762 & -202.8 & .15799073 & 4.2741343 \\
\hline-207.7 & .13719572 & 4.2084583 & -202.7 & 15841819 & 4.2751700 \\
\hline-207.6 & .13761665 & 4.2101253 & -202.6 & 15884576 & 4.2761949 \\
\hline-207.5 & .13803775 & 4.2117772 & -202.5 & .15927343 & 4.2772091 \\
\hline-207.4 & .13845901 & 4.2134141 & -202.4 & .15970120 & 4.2782126 \\
\hline-207.3 & .13888043 & 4.2150361 & -202.3 & .16012907 & 4.2792055 \\
\hline-207.2 & .13930201 & 4.2166434 & -202.2 & .16055704 & 4.2801879 \\
\hline-207.1 & .13972376 & 4.2182360 & -202.1 & .16098511 & 4.2811598 \\
\hline-207.0 & .14014566 & 4.2198140 & -202.0 & .16141328 & 4.2821214 \\
\hline-206.9 & .14056772 & 4.2213775 & -201.9 & .16184154 & 4.2830727 \\
\hline-206.8 & .14098994 & 4.2229266 & -201.8 & .16226989 & 4.2840138 \\
\hline-206.7 & .14141231 & 4.2244615 & -201.7 & .16269834 & 4.2849447 \\
\hline-206.6 & .14183483 & 4.2259821 & -201.6 & .16312688 & 4.2858655 \\
\hline-206.5 & .14225750 & 4.2274887 & -201.5 & 16355551 & 4.2867764 \\
\hline-206.4 & .14268032 & 4.2289812 & -201.4 & 16398423 & 4.2876773 \\
\hline-206.3 & .14310330 & 4.2304598 & -201.3 & 16441305 & 4.2885683 \\
\hline-206.2 & .14352642 & 4.2319246 & -201.2 & 16484195 & 4.2894495 \\
\hline-206.1 & .14394968 & 4.2333757 & -201.1 & .16527094 & 4.2903210 \\
\hline-206.0 & .14437309 & 4.2348131 & -201.0 & 16570001 & 4.2911828 \\
\hline-205.9 & 14479664 & 4.2362370 & -200.9 & 16612917 & 4.2920350 \\
\hline-205.8 & 14522034 & 4.2376474 & -200.8 & 16655842 & 4.2928777 \\
\hline-205.7 & 14564417 & 4.2390445 & -200.7 & .16698775 & 4.2937109 \\
\hline-205.6 & 14606815 & 4.2404283 & -200.6 & 16741716 & 4. 2945347 \\
\hline-205.5 & 14649226 & 4. 2417989 & -200.5 & 16784665 & 4.2953492 \\
\hline-205.4 & 14691651 & 4.2431564 & -200.4 & 16827623 & 4. 2961544 \\
\hline-205.3 & 14734089 & 4. 2445009 & -200.3 & 16870588 & 4.2969504 \\
\hline-205.2 & .14776541 & 4.2458324 & -200.2 & 16913562 & 4.2977373 \\
\hline-205.1 & 14819005 & 4.2471512 & -200.1 & 16956543 & 4.2985150 \\
\hline-205.0 & 14861484 & 4. 2484572 & -200.0 & 16999532 & 4.2992838 \\
\hline
\end{tabular}


IPTS-68 Reference Function and Its First Derivative With the Argument in Celsius - Continued

\begin{tabular}{|c|c|c|c|c|c|}
\hline$\stackrel{t}{{ }^{\circ} \mathrm{C}}$ & $W^{*}(t)$ & $\begin{array}{c}d W^{*}(t) / d T \\
\times 1000 \\
{ }^{\circ} \mathrm{C}^{-1}\end{array}$ & ${ }^{\circ} \mathrm{C}$ & $W^{*}(t)$ & $\begin{array}{c}d W^{*}(t) / d T \\
\times \quad 1000 \\
{ }^{\circ} \mathrm{C}^{-1}\end{array}$ \\
\hline 200.0 & 16999532 & 4.2992838 & -195.0 & 19157018 & 4. 3275324 \\
\hline-199.9 & .17042529 & 4.3000436 & -194.9 & .19200296 & 4.3279174 \\
\hline-199.8 & 17085533 & 4.3007946 & -194.8 & 19243577 & 4. 3282962 \\
\hline-199.7 & .17128545 & 4.3015367 & -194.7 & 19286862 & 4.3286688 \\
\hline-199.6 & .17171564 & 4.3022701 & -194.6 & 19330150 & 4.3290353 \\
\hline-199.5 & 17214590 & 4.3029948 & -194.5 & 19373442 & 4.3293958 \\
\hline-199.4 & .17257624 & 4.3037108 & -194.4 & 19416738 & 4.3297503 \\
\hline-199.3 & .17300664 & 4.3044183 & -194.3 & 19460037 & 4.3300987 \\
\hline-199.2 & .17343712 & 4.3051172 & -194.2 & 19503340 & 4.3304413 \\
\hline-199.1 & .17386767 & 4.3058077 & -194.1 & 19546646 & 4.3307780 \\
\hline-199.0 & .17429828 & 4.3064898 & -194.0 & 19589955 & 4.3311088 \\
\hline-198.9 & 17472896 & 4.3071636 & -193.9 & 19633268 & 4.3314337 \\
\hline-198.8 & .17515971 & 4.3078291 & -193.8 & 19676584 & 4.3317530 \\
\hline-198.7 & .17559053 & 4.3084864 & -193.7 & 19719903 & 4.3320665 \\
\hline-198.6 & .17602141 & 4.3091354 & -193.6 & .19763225 & 4.3323744 \\
\hline-198.5 & .17645236 & 4.3097765 & -193.5 & 19806551 & 4.3326766 \\
\hline-198.4 & .17688336 & 4.3104094 & -193.4 & 19849879 & 4.3329732 \\
\hline-198.3 & .17731444 & 4.3110344 & -193.3 & 19893210 & 4.3332643 \\
\hline-198.2 & .17774557 & 4.3116515 & -193.2 & 19936544 & 4.3335498 \\
\hline-198.1 & .17817677 & 4.3122607 & -193.1 & 19979881 & 4.3338299 \\
\hline-198.0 & .17860802 & 4.3128620 & -193.0 & 20023221 & 4.3341046 \\
\hline-197.9 & .17903934 & 4.3134557 & -192.9 & .20066563 & 4.3343739 \\
\hline-197.8 & .17947071 & 4.3140415 & -192.8 & 20109908 & 4.3346378 \\
\hline-197.7 & .17990215 & 4.3146198 & -192.7 & 20153256 & 4.3348964 \\
\hline-197.6 & .18033364 & 4.3151904 & -192.6 & .20196606 & 4.3351498 \\
\hline-197.5 & 18076519 & 4.3157535 & -192.5 & .20239959 & 4.3353979 \\
\hline-197.4 & .18119679 & 4.3163092 & -192.4 & .20283314 & 4.3356408 \\
\hline-197.3 & 18162845 & 4.3168574 & -192.3 & 20326672 & 4.3358786 \\
\hline-197.2 & .18206016 & 4.3173982 & -192.2 & 20370032 & 4.3361112 \\
\hline-197.1 & 18249193 & 4.3179317 & -192.1 & 20413394 & 4.3363388 \\
\hline-197.0 & .18292375 & 4.3184579 & -192.0 & 20456758 & 4.3365614 \\
\hline-196.9 & .18335562 & 4.3189768 & -191.9 & 20500125 & 4.3367789 \\
\hline-196.8 & .18378754 & 4. 3194887 & -191.8 & 20543494 & 4.3369915 \\
\hline-1967 & .18421952 & 4.3199934 & -191.7 & 20586865 & 4.3371991 \\
\hline-196.6 & .18465154 & 4.3204910 & -191.6 & 20630238 & 4.3374019 \\
\hline-196.5 & 18508361 & 4.3209816 & -191.5 & 20673613 & 4.3375999 \\
\hline-196.4 & 18551574 & 4.3214653 & -191.4 & 20716990 & 4.3377930 \\
\hline-196.3 & .18594791 & 4.3219420 & -191.3 & 20760369 & 4.3379813 \\
\hline-196.2 & .18638012 & 4.3224118 & -191.2 & 20803750 & 4.3381649 \\
\hline-196.1 & . 18681239 & 4.3228749 & -191.1 & .20847132 & 4.3383437 \\
\hline-196.0 & .18724470 & 4.3233312 & -191.0 & 20890516 & 4.3385180 \\
\hline-195.9 & . 18767705 & 4.3237807 & -190.9 & 20933902 & 4.3386875 \\
\hline-195.8 & 18810945 & 4.3242236 & -190.8 & .20977290 & 4.3388525 \\
\hline-195.7 & . 18854790 & 4.3246599 & -190.7 & 21020680 & 4.3390129 \\
\hline-195.6 & .18897439 & 4.3250896 & -190.6 & 21064070 & 4.3391688 \\
\hline-195.5 & . 18940692 & 4.3255128 & -190.5 & .21107463 & 4.3393202 \\
\hline-195.4 & .18983949 & 4.3259294 & -190.4 & 21150857 & 4.3394671 \\
\hline-195.3 & .19027210 & 4.3263397 & -190.3 & 21194252 & 4.3396095 \\
\hline-195.2 & .19070476 & 4.3267436 & -190.2 & 21237649 & 4.3397477 \\
\hline-195.1 & .19113745 & 4.3271412 & -190.1 & 21281047 & 4.3398814 \\
\hline-195.0 & .19157018 & 4.3275324 & -190.0 & 21324447 & 4.3400108 \\
\hline
\end{tabular}


IPTS-68 Reference Function and Its First Derivative With the Argument in Celsius - Continued

\begin{tabular}{|c|c|c|c|c|c|}
\hline${ }^{\circ} \mathrm{C}$ & $W^{*}(t)$ & $\begin{array}{c}d W^{*}(t) / d T \\
\times 1000 \\
{ }^{\circ} \mathrm{C}^{-1}\end{array}$ & $\stackrel{t}{{ }^{\circ} \mathrm{C}}$ & $W^{*}(t)$ & $\begin{array}{c}d W^{*}(t) / d T \\
\times 1000 \\
{ }^{\circ} \mathrm{C}^{-1}\end{array}$ \\
\hline-190.0 & .21324447 & 4.3400108 & -185.0 & .23495228 & 4.3416391 \\
\hline-189.9 & .21367847 & 4.3401359 & -184.9 & .23538645 & 4. 3415872 \\
\hline-189.8 & .21411249 & 4.3402567 & -184.8 & .23582060 & 4.3415325 \\
\hline-189.7 & .21454652 & 4.3403733 & -184.7 & .23625475 & 4.3414750 \\
\hline-189.6 & .21498057 & 4. 3404857 & -184.6 & .23668890 & 4.3414145 \\
\hline-189.5 & .21541462 & 4.3405940 & -184.5 & .23712303 & 4.3413514 \\
\hline-189.4 & .21584869 & 4.3406981 & -184.4 & .23755717 & 4.3412854 \\
\hline-189.3 & .21628276 & 4.3407981 & -184.3 & .23799129 & 4.3412168 \\
\hline-189.2 & .21671685 & 4.3408941 & -184.2 & .23842541 & 4.3411453 \\
\hline-189.1 & .21715094 & 4.3409860 & -184.1 & 23885952 & 4.3410712 \\
\hline-189.0 & .21758504 & 4.3410739 & -184.0 & 23929362 & 4.3409944 \\
\hline-188.9 & .21801915 & 4.3411579 & -183.9 & 23972772 & 4.3409150 \\
\hline-188.8 & .21845327 & 4.3412378 & -183.8 & .24016181 & 4.3408329 \\
\hline-188.7 & .21888740 & 4.3413139 & -183.7 & .24059589 & 4.3407482 \\
\hline-188.6 & .21932154 & 4.3413861 & -183.6 & .24102996 & 4.3406609 \\
\hline-188.5 & .21975568 & 4.3414544 & -183.5 & .24146402 & 4.3405710 \\
\hline-188.4 & .22018983 & 4.3415189 & -183.4 & .24189807 & 4.3404786 \\
\hline-188.3 & . 22062398 & 4.3415796 & -183.3 & .24233211 & 4.3403836 \\
\hline-188.2 & .22105814 & 4.3416366 & -183.2 & .24276615 & 4.3402862 \\
\hline-188.1 & .22149231 & 4.3416898 & -183.1 & .24320017 & 4.3401862 \\
\hline-188.0 & .22192648 & 4.3417393 & -183.0 & .24363418 & 4.3400839 \\
\hline-187.9 & .22236066 & 4.3417852 & -182.9 & .24406819 & 4.3399790 \\
\hline-187.8 & .22279484 & 4.3418274 & -182.8 & .24450218 & 4.3398718 \\
\hline-187.7 & .22322902 & 4.3418659 & -182.7 & .24493616 & 4.3397620 \\
\hline-187.6 & .22366321 & 4.3419009 & -182.6 & .24537013 & 4.3396500 \\
\hline-187.5 & .22409740 & 4.3419323 & -182.5 & .24580409 & 4.3395356 \\
\hline-187.4 & .22453160 & 4.3419602 & -182.4 & .24623804 & 4.3394188 \\
\hline-187.3 & .22496579 & 4.3419846 & -182.3 & .24667198 & 4.3392998 \\
\hline-187.2 & .22539999 & 4.3420055 & -182.2 & .24710590 & 4.3391784 \\
\hline-187.1 & .22583420 & 4.3420229 & -182.1 & 24753981 & 4.3390548 \\
\hline-187.0 & .22626840 & 4.3420369 & -182.0 & 24797371 & 4.3389288 \\
\hline-186.9 & . 22670260 & 4.3420476 & -181.9 & .24840760 & 4.3388007 \\
\hline-186.8 & .22713681 & 4.3420548 & -181.8 & .24884147 & 4.3386703 \\
\hline-186.7 & .22757101 & 4.3420587 & -181.7 & .24927533 & 4.3385378 \\
\hline-186.6 & .22800522 & 4.3420593 & -181.6 & .24970918 & 4.3384029 \\
\hline-186.5 & .22843943 & 4.3420566 & -181.5 & .25014301 & 4.3382660 \\
\hline-186.4 & .22887363 & 4.3420507 & -181.4 & .25057683 & 4.3381270 \\
\hline-186.3 & .22930784 & 4.3420415 & -181.3 & .25101064 & 4.3379858 \\
\hline-186.2 & .22974204 & 4.3420291 & -181.2 & .25144443 & 4.3378425 \\
\hline-186.1 & .23017624 & 4.3420135 & -181.1 & .25187820 & 4.3376971 \\
\hline-186.0 & .23061044 & 4.3419948 & -181.0 & .25231197 & 4.3375496 \\
\hline-185.9 & .23104464 & 4.3419729 & -180.9 & .25274571 & 4.3374001 \\
\hline-185.8 & .23147884 & 4.3419479 & -180.8 & .25317945 & 4.3372485 \\
\hline-185.7 & .23191303 & 4.3419198 & -180.7 & .25361316 & 4.3370949 \\
\hline-185.6 & .23234722 & 4.3418887 & -180.6 & .25404687 & 4.3369394 \\
\hline-185.5 & .23278141 & 4.3418545 & -180.5 & .25448055 & 4.3367818 \\
\hline-185.4 & .23321559 & 4.3418173 & -180.4 & .25491422 & 4. 3366223 \\
\hline-185.3 & .23364977 & 4.3417772 & -180.3 & .25534788 & 4.3364608 \\
\hline-185.2 & .23408395 & 4.3417341 & -180.2 & .25578151 & 4.3362975 \\
\hline-185.1 & .23451812 & 4.3416881 & -180.1 & .25621514 & 4.3361321 \\
\hline-185.0 & 23495228 & 4.3416391 & -180.0 & .25664874 & 4.3359649 \\
\hline
\end{tabular}


IPTS-68 Reference Function and Its First Derivative With the Argument in Celsius - Continued

\begin{tabular}{|c|c|c|c|c|c|}
\hline${ }^{t} \mathrm{C}$ & $W^{*}(t)$ & $\begin{array}{c}d W^{*}(t) / d T \\
\quad \times 1000 \\
{ }^{\circ} \mathrm{C}^{-1}\end{array}$ & ${ }^{t} \mathrm{C}$ & $W^{*}(t)$ & $\begin{array}{c}d W^{*}(t) / d T \\
\times 1000 \\
{ }^{\circ} \mathrm{C}^{-1}\end{array}$ \\
\hline-180.0 & .25664874 & 4.3359649 & -175.0 & 27830405 & 4.3255391 \\
\hline-179.9 & .25708233 & 4.3357959 & -174.9 & 27873660 & 4. 3252957 \\
\hline-179.8 & .25751590 & 4.3356249 & -174.8 & 27916911 & 4.3250511 \\
\hline-179.7 & .25794945 & 4.3354522 & -174.7 & 27960161 & 4.3248053 \\
\hline-179.6 & .25838299 & 4.3352775 & -174.6 & 28003407 & 4.3245585 \\
\hline-179.5 & .25881651 & 4.3351011 & -174.5 & 28046652 & 4.3243105 \\
\hline-179.4 & .25925001 & 4.3349229 & -174.4 & 28089894 & 4.3240615 \\
\hline-179.3 & .25968349 & 4.3347430 & -174.3 & 28133133 & 4.3238114 \\
\hline-179.2 & .26011696 & 4.3345612 & -174.2 & 28176370 & 4.3235602 \\
\hline-179.1 & .26055041 & 4.3343777 & -174.1 & 28219604 & 4.3233079 \\
\hline-179.0 & 26098383 & 4.3341925 & -174.0 & .28262836 & 4.3230546 \\
\hline-178.9 & .26141724 & 4.3340056 & -173.9 & .28306055 & 4.3228003 \\
\hline-178.8 & 26185064 & 4.3338169 & -173.8 & .28349292 & 4.3225448 \\
\hline-178.7 & .26228401 & 4.3336267 & -173.7 & .28392516 & 4.3222885 \\
\hline-178.6 & .26271736 & 4.3334347 & -173.6 & .28435738 & 4.3220311 \\
\hline-178.5 & .26315069 & 4.3332411 & -173.5 & .28478957 & 4.3217727 \\
\hline-178.4 & 26358401 & 4.3330459 & -173.4 & .28522173 & 4.3215133 \\
\hline-178.3 & .26401730 & 4.3328489 & -173.3 & .28565387 & 4.3212530 \\
\hline-178.2 & 26445058 & 4.3326505 & -173.2 & .28608598 & 4.3209917 \\
\hline-178.1 & 26488383 & 4.3324505 & -173.1 & .28651807 & 4.3207295 \\
\hline-178.0 & .26531707 & 4.3322488 & -173.0 & .28695013 & 4.3204663 \\
\hline-177.9 & .26575028 & 4.3320456 & -172.9 & .28738216 & 4.3202022 \\
\hline-177.8 & 26618348 & 4.3318409 & -172.8 & .28781417 & 4.3199371 \\
\hline-177.7 & 26661665 & 4.3316346 & -172.7 & .28824615 & 4.3196712 \\
\hline-177.6 & 26704980 & 4.3314269 & -172.6 & .28867810 & 4.3194044 \\
\hline-177.5 & 26748294 & 4.3312176 & -172.5 & .28911003 & 4.3191366 \\
\hline-177.4 & 26791605 & 4.3310068 & -172.4 & .28954193 & 4.3188680 \\
\hline-177.3 & 26834914 & 4.3307946 & -172.3 & 28997380 & 4.3185986 \\
\hline-177.2 & 26878221 & 4.3305809 & -172.2 & .29040565 & 4.3183283 \\
\hline-177.1 & 26921525 & 4.3303658 & -172.1 & .29083747 & 4.3180571 \\
\hline-177.0 & 26964828 & 4.3301492 & -172.0 & 29126926 & 4.3177851 \\
\hline-176.9 & 27008128 & 4.3299313 & -171.9 & .29170103 & 4.3175123 \\
\hline-176.8 & 27051427 & 4.3297119 & -171.8 & .29213276 & 4.3172386 \\
\hline-176.7 & 27094723 & 4.3294912 & -171.7 & .29256447 & 4.3169642 \\
\hline-176.6 & 27138016 & 4.3292691 & -171.6 & .29299616 & 4.3166889 \\
\hline-176.5 & 27181308 & 4.3290455 & -171.5 & .29342781 & 4.3164129 \\
\hline-176.4 & 27224597 & 4.3288207 & -171.4 & .29385944 & 4.3161361 \\
\hline-176.3 & 27267884 & 4.3285945 & -171.3 & .29429104 & 4.3158585 \\
\hline-176.2 & 27311169 & 4.3283671 & -171.2 & .29472261 & 4.3155801 \\
\hline-176.1 & 27354452 & 4.3281383 & -171.1 & .29515416 & 4.3153010 \\
\hline-176.0 & 27397732 & 4.3279082 & -171.0 & .29558567 & 4.3150212 \\
\hline-175.9 & 27441010 & 4.3276768 & -170.9 & .29601716 & 4.3147407 \\
\hline-175.8 & 27484286 & 4.3274442 & -170.8 & 29644862 & 4.3144593 \\
\hline-175.7 & 27527559 & 4.3272103 & -170.7 & .29688005 & 4.3141774 \\
\hline-175.6 & 27570830 & 4.3269752 & -170.6 & .29731146 & 4.3138947 \\
\hline-175.5 & 27614098 & 4.3267389 & -170.5 & .29774283 & 4.3136113 \\
\hline-175.4 & 27657365 & 4.3265013 & -170.4 & .29817418 & 4.3133271 \\
\hline-175.3 & 27700628 & 4.3262625 & -170.3 & .29860550 & 4.3130424 \\
\hline-175.2 & 27743890 & 4.3260226 & -170.2 & .29903679 & 4.3127569 \\
\hline-175.1 & 27787149 & 4.3257814 & -170.1 & .29946805 & 4.3124708 \\
\hline-175.0 & 27830405 & 4.3255391 & -170.0 & 29989928 & 4.3121841 \\
\hline
\end{tabular}


IPTS-68 Reference Function and Its First Derivative With the Argument in Celsius-Continued

\begin{tabular}{|c|c|c|c|c|c|}
\hline${ }^{t} \mathrm{C}$ & $W^{*}(t)$ & $\begin{array}{c}d W^{*}(t) / d T \\
\times 1000 \\
{ }^{\circ} \mathrm{C}^{-1}\end{array}$ & $\stackrel{t}{{ }^{t} \mathrm{C}}$ & $W^{*}(t)$ & $\begin{array}{c}d W^{*}(t) / d T \\
\times 1000 \\
{ }^{\circ} \mathrm{C}^{-1}\end{array}$ \\
\hline-170.0 & . 29989928 & 4.3121841 & -120.0 & .51175723 & 4.1696979 \\
\hline-169.0 & .30421002 & 4.3092826 & -119.0 & .51592580 & 4.1674503 \\
\hline-168.0 & .30851783 & 4.3063249 & -118.0 & .52009214 & 4.1652272 \\
\hline-167.0 & .31282265 & 4.3033185 & -117.0 & .52425626 & 4.1630283 \\
\hline-166.0 & .31712445 & 4.3002706 & -116.0 & .52841820 & 4.1608530 \\
\hline-165.0 & .32142318 & 4.2971877 & -115.0 & .53257798 & 4.1587009 \\
\hline-164.0 & .32571882 & 4.2940758 & -114.0 & .53673561 & 4.1565715 \\
\hline-163.0 & .33001133 & 4.2909406 & -113.0 & .54089113 & 4.1544643 \\
\hline-162.0 & .33430069 & 4.2877872 & -112.0 & .54504455 & 4.1523789 \\
\hline-161.0 & .33858690 & 4.2846206 & -111.0 & 54919589 & 4.1503148 \\
\hline-160.0 & .34286993 & 4.2814449 & -110.0 & .55334518 & 4.1482715 \\
\hline-159.0 & .34714978 & 4.2782645 & -109.0 & .55749244 & 4.1462487 \\
\hline-158.0 & .35142646 & 4.2750828 & -108.0 & .56163769 & 4.1442458 \\
\hline-157.0 & .35569995 & 4.2719035 & -107.0 & .56578094 & 4.1422623 \\
\hline-156.0 & .35997027 & 4.2687295 & -106.0 & .56992222 & 4.1402979 \\
\hline-155.0 & .36423741 & 4.2655639 & -105.0 & .57406154 & 4.1383521 \\
\hline-154.0 & .36850140 & 4.2624092 & -104.0 & .57819893 & 4.1364243 \\
\hline-153.0 & .37276224 & 4.2592676 & -103.0 & .58233440 & 4.1345144 \\
\hline-152.0 & .37701994 & 4.2561416 & -102.0 & .58646796 & 4.1326217 \\
\hline-151.0 & .38127452 & 4.2530330 & -101.0 & 59059965 & 4.1307459 \\
\hline-150.0 & .38552601 & 4.2499434 & -100.0 & 59472946 & 4.1288866 \\
\hline-149.0 & .38977442 & 4.2468745 & -99.0 & .59885742 & 4.1270433 \\
\hline-148.0 & .39401977 & 4.2438278 & -98.0 & 60298355 & 4.1252157 \\
\hline-147.0 & .39826208 & 4.2408044 & -97.0 & 60710786 & 4.1234034 \\
\hline-146.0 & .40250138 & 4.2378054 & -96.0 & 61123036 & 4.1216060 \\
\hline-145.0 & .40673770 & 4.2348319 & -95.0 & 61535108 & 4.1198231 \\
\hline-144.0 & .41097106 & 4.2318846 & -94.0 & 61947002 & 4.1180544 \\
\hline-143.0 & .41520148 & 4.2289643 & -93.0 & 62358719 & 4.1162996 \\
\hline-142.0 & .41942899 & 4.2260716 & -92.0 & 62770262 & 4.1145582 \\
\hline-141.0 & .42365363 & 4.2232070 & -91.0 & 63181631 & 4.1128299 \\
\hline-140.0 & .42787542 & 4.2203709 & -90.0 & 63592828 & 4.1111145 \\
\hline-139.0 & .43209438 & 4.2175636 & -89.0 & 64003855 & 4.1094117 \\
\hline-138.0 & .43631056 & 4.2147855 & -88.0 & 64414711 & 4.1077210 \\
\hline-137.0 & .44052396 & 4.2120366 & -87.0 & 64825399 & 4.1060422 \\
\hline-136.0 & .44473464 & 4.2093170 & -86.0 & 65235920 & 4.1043750 \\
\hline-135.0 & .44894261 & 4.2066270 & -85.0 & .65646275 & 4.1027192 \\
\hline-134.0 & .45314790 & 4.2039663 & -84.0 & 66056464 & 4.1010745 \\
\hline-133.0 & .45735055 & 4.2013350 & -83.0 & 66466490 & 4.0994405 \\
\hline-132.0 & .46155058 & 4.1987329 & -82.0 & 66876353 & 4.0978171 \\
\hline-131.0 & .46574803 & 4.1961599 & -81.0 & 67286054 & 4.0962040 \\
\hline-130.0 & .46994291 & 4.1936158 & -80.0 & 67695594 & 4.0946010 \\
\hline-129.0 & .47413527 & 4.1911002 & -79.0 & 68104974 & 4.0930078 \\
\hline-128.0 & .47832512 & 4.1886132 & -78.0 & .68514196 & 4.0914242 \\
\hline-127.0 & .48251250 & 4.1861542 & -77.0 & 68923259 & 4.0898500 \\
\hline-126.0 & .48669744 & 4.1837229 & -76.0 & .69332166 & 4.0882850 \\
\hline-125.0 & .49087996 & 4.1813191 & -75.0 & 69740917 & 4.0867290 \\
\hline-124.0 & .49506009 & 4.1789423 & -74.0 & 70149512 & 4.0851817 \\
\hline-123.0 & .49923785 & 4.1765922 & -73.0 & .70557953 & 4.0836431 \\
\hline-122.0 & .50341328 & 4.1742684 & -72.0 & 70966241 & 4.0821129 \\
\hline-121.0 & .50758640 & 4.1719704 & -71.0 & .71374376 & 4.0805910 \\
\hline-120.0 & .51175723 & 4.169679 & -70.0 & .71782359 & 4.0790772 \\
\hline
\end{tabular}


IPTS-68 Reference Function and Its Firts Derivative With the Argument in Celsius-Continued

\begin{tabular}{|c|c|c|c|c|c|}
\hline$\stackrel{t}{{ }^{t} \mathrm{C}}$ & $W^{*}(t)$ & $\begin{array}{c}d W^{*}(t) / d T \\
\times 1000 \\
{ }^{\circ} \mathrm{C}^{-1}\end{array}$ & ${ }^{t} \mathrm{C}$ & $W^{*}(t)$ & $\begin{array}{c}d W^{*}(t) / d T \\
\times 1000 \\
{ }^{\circ} \mathrm{C}^{-1} \\
\end{array}$ \\
\hline-70.0 & .71782359 & 4.0790772 & -20.0 & 92002439 & 4.0111301 \\
\hline-69.0 & .72190192 & 4.0775712 & -19.0 & 92403489 & 4.0098834 \\
\hline-68.0 & .72597874 & 4.0760731 & -18.0 & 92804416 & 4.0086393 \\
\hline-67.0 & 73005407 & 4.0745825 & -17.0 & 93205217 & 4.0073976 \\
\hline-66.0 & .73412791 & 4.0730995 & -16.0 & 93605895 & 4.0061582 \\
\hline-65.0 & .73820027 & 4.0716238 & -15.0 & 94006449 & 4.0049209 \\
\hline-64.0 & .74227116 & 4.0701553 & -14.0 & 94406879 & 4.0036856 \\
\hline-63.0 & 74634058 & 4.0686939 & -13.0 & 94807186 & 4.0024522 \\
\hline-62.0 & .75040855 & 4.0672394 & -12.0 & 95207370 & 4. 012205 \\
\hline-61.0 & .75447506 & 4.0657918 & -11.0 & 95607430 & 3.9999902 \\
\hline-60.0 & .75854013 & 4.0643509 & -10.0 & 96007368 & 3.9987614 \\
\hline-59.0 & .76260377 & 4.0629166 & -9.0 & 96407183 & 3.9975337 \\
\hline-58.0 & .76666597 & 4.0614887 & -8.0 & 96806875 & 3.9963070 \\
\hline-57.0 & .77072675 & 4.0600673 & -7.0 & 97206444 & 3.9950811 \\
\hline-56.0 & .77478611 & 4.0586521 & -6.0 & 97605891 & 3.9938559 \\
\hline-55.0 & .77884405 & 4.0572432 & -5.0 & 98005215 & 3.9926310 \\
\hline-54.0 & .78290059 & 4.0558403 & -4.0 & 98404417 & 3.9914065 \\
\hline-53.0 & .78695573 & 4.0544434 & -3.0 & 98803497 & 3.9901819 \\
\hline-52.0 & .79100948 & 4.0530523 & -2.0 & 99202454 & 3.9889571 \\
\hline-51.0 & .79506184 & 4.0516672 & -1.0 & 99601288 & 3.9877321 \\
\hline-50.0 & 79911282 & 4.0502877 & .0 & 1.00000000 & 3.9865063 \\
\hline-49.0 & 80316242 & 4.0489138 & & & \\
\hline-48.0 & 80721065 & 4.0475455 & & & \\
\hline-47.0 & .81125751 & 4.0461827 & & & \\
\hline-46.0 & .81530302 & 4.0448252 & & & \\
\hline-45.0 & .81934716 & 4.0434730 & & & \\
\hline-44.0 & 82338996 & 4.0421259 & & & \\
\hline-43.0 & 82743142 & 4.0407841 & & & \\
\hline-42.0 & .83147153 & 4.0394472 & & & \\
\hline-41.0 & .83551031 & 4.0381154 & & & \\
\hline-40.0 & .83954777 & 4.0367884 & & & \\
\hline-39.0 & 84358389 & 4.0354661 & & & \\
\hline-38.0 & 84761870 & 4.0341486 & & & \\
\hline-37.0 & 85165219 & 4.0328357 & & & \\
\hline-36.0 & 85568437 & 4.0315273 & & & \\
\hline-35.0 & .85971525 & 4.0302233 & & & \\
\hline-34.0 & 86374482 & 4.0289237 & & & \\
\hline-33.0 & 86777310 & 4.0276283 & & & \\
\hline-32.0 & .87180008 & 4.0263371 & & & \\
\hline-31.0 & 87582577 & 4.0250500 & & & \\
\hline-30.0 & 87985018 & 4.0237668 & & & \\
\hline-29.0 & 88387331 & 4.0224875 & & & \\
\hline-28.0 & 88789516 & 4.0212120 & & & \\
\hline-27.0 & .89191573 & 4.0199401 & & & \\
\hline-26.0 & 89593504 & 4.0186718 & & & \\
\hline-25.0 & 89995308 & 4.0174069 & & & \\
\hline-24.0 & .90396985 & 4.0161454 & & & \\
\hline-23.0 & 90798537 & 4.0148870 & & & \\
\hline-22.0 & .91199963 & 4.0136318 & & & \\
\hline-21.0 & 91601263 & 4.0123795 & & & \\
\hline-20.0 & 92002439 & 4.0111301 & & & \\
\hline
\end{tabular}




\section{APPEndix F. Analysis of the First Derivatives at $0{ }^{\circ} \mathrm{C}$ of IPTS-68 Platinum Resistance Thermometer Formulations Above and Below $0{ }^{\circ} \mathrm{C}$}

The four specified deviation functions of the IPTS -68 in the interval $13.81 \mathrm{~K}$ to $273.15 \mathrm{~K}$ are formulated to join smoothly by constraining the values of the first derivatives of the formulations above and below each point of joining to be identical at this point (see the IPTS-68 document). However, the values of the first derivatives of the formulations above and below $0{ }^{\circ} \mathrm{C}$ of real SPRT's are not constrained to be equal at $0^{\circ} \mathrm{C}$. This discussion will show that the magnitude of this difference is negligible for SPRT's that have suitable thermometer constants. All SPRT's received at the NBS for calibration in the past few years had suitable constants (see the last page of this Appendix).

The IPTS-68 reference function in Celsius

$$
t=\sum_{i=1}^{20} A_{i}\left[\ln W^{*}(t)\right]^{i}{ }^{\circ} \mathrm{C}
$$

was formulated so that its first and second derivatives would have the same value at $0{ }^{\circ} \mathrm{C}$ as obtained from

$$
t=t^{\prime}+0.045\left(\frac{t^{\prime}}{s}\right)\left(\frac{t^{\prime}}{s}-1\right)\left(\frac{t^{\prime}}{z}-1\right)\left(\frac{t^{\prime}}{a}-1\right){ }^{\circ} \mathrm{C}
$$

and

$$
t^{\prime}=\frac{1}{\alpha}\left[W\left(t^{\prime}\right)-1\right]+\delta\left(\frac{t^{\prime}}{s}\right)\left(\frac{t^{\prime}}{s}-1\right){ }^{\circ} \mathrm{C}
$$

with thermometer constants

$$
\alpha=3.9259668 \times 10^{-3} /{ }^{\circ} \mathrm{C}
$$

and

$$
\delta=1.496334^{\circ} \mathrm{C} \text {. }
$$

(The above selected values of $\alpha$ and $\delta$ given in the text of the IPTS-68 will, henceforth, be referred to as $\alpha^{*}$ and $\delta^{*}$, respectively. In eqs (1), (2), and (3), $W^{*}(t)=W_{\text {CCT-68 }}\left(t_{68}\right)$ and $t=t_{68}$ (for convenience), $s=100^{\circ} \mathrm{C}, z=419.58{ }^{\circ} \mathrm{C}, a=630.74{ }^{\circ} \mathrm{C}$, and $W\left(t^{\prime}\right)=$ $\boldsymbol{H}^{\prime}(t)=R(t) / R(0)$.) The second term of eq (2) represents a "correction" to be made to the temperature value $t^{\prime}$ to obtain a temperature value $t$ that is a closer approximation to the thermodynamic scale. The values $t^{\prime}$ and $t$ represent the same temperature (hotness). For real SPRT's the derivative of the formulation between $-182.962{ }^{\circ} \mathrm{C}$ and $0{ }^{\circ} \mathrm{C}$ is obtained from the definition of the IPTS-68 in this temperature range

$$
W(t)=W^{*}(t)+\Delta W(t)
$$

and the deviation function

$$
\Delta W(t)=A_{4} t+C_{4} t^{3}(t-100) .
$$

The constants of eq (5) are determined from the deviations at the oxygen point and the steam point. When differentiated with respect to $t$, there results from eqs (4) and (5), respectively,

$$
\frac{d W(t)}{d t}=\frac{d W^{*}(t)}{d t}+\frac{d \Delta W(t)}{d t}
$$

and

$$
\frac{d \Delta W(t)}{d t}=A_{4}+4 C_{4} t^{2}(t-75) .
$$

At $0{ }^{\circ} \mathrm{C}$, the reference function (eq (1)) on differentiation yields

$$
\left(\frac{d W^{*}(t)}{d t}\right)_{0}=\frac{1}{A_{1}}
$$

and eq (7) yields

$$
\left(\frac{d \Delta W(t)}{d t}\right)_{0}=A_{4}
$$

When eqs (6), (8), and (9) are combined, there results

$$
\left(\frac{d W(t)}{d t}\right)_{0 \mathrm{~B}}=\frac{1}{A_{1}}+A_{4},
$$

where the subscript $\mathrm{OB}$ refers to the formulation below $0{ }^{\circ} \mathrm{C}$. The constant $A_{4}$ is obtained from the value of $W\left(100^{\circ} \mathrm{C}\right)$, i.e., from

$$
W\left(100^{\circ} \mathrm{C}\right)=100 \alpha+1
$$

and

$$
W^{*}\left(100{ }^{\circ} \mathrm{C}\right)=100 \alpha^{*}+1 .
$$

The value of $\alpha$ in eq (11) can be obtained from measurements at the triple point of water and the steam point or at the triple point of water, tin point, and zinc point $(\alpha=[R(100)-R \quad 0) / 100 R(0))$. Combining equations (11) and (12) with eq (5) $(t=$ $100^{\circ} \mathrm{C}$ ) there results

$$
A_{4}=\alpha-\alpha^{*} .
$$

When eq (10) and (13) are combined,

$$
\left(\frac{d W(t)}{d t}\right)_{\mathrm{OB}}=\frac{1}{A_{1}}+\alpha-\alpha^{*} .
$$


The derivative of the formulation between 0 and $630.74{ }^{\circ} \mathrm{C}$ is obtained by differentiating eq (2) and the equation

$$
W\left(t^{\prime}\right)=1+A t^{\prime}+B t^{\prime 2}
$$

there results, respectively,

$$
\begin{aligned}
\frac{d t}{d t^{\prime}}=1+\frac{0.045}{s^{2} z a}[ & 4 t^{\prime 3}-3(s+z+a) t^{\prime 2} \\
& \left.+2(s z+s a+z a) t^{\prime}-s z a\right],
\end{aligned}
$$

and

$$
\frac{d W(t)}{d t^{\prime}}=A+2 B t^{\prime}
$$

Equation (15) is equivalent to eq (3) with the thermometer constants $A=\alpha\left(1+\delta / 100{ }^{\circ} \mathrm{C}\right)$ and $B=$ $-10^{-4} \alpha \delta{ }^{\circ} \mathrm{C}^{-2}$. At $t=0{ }^{\circ} \mathrm{C}, t^{\prime}=0^{\circ} \mathrm{C}$, and eqs (16) and (17) become respectively,

$$
\left(\frac{d t}{d t^{\prime}}\right)_{0}=1-\frac{0.045}{100}=0.99955
$$

and

$$
\left(\frac{d W(t)}{d t^{\prime}}\right)_{0}=A
$$

Combining eqs (18) and (19),

$$
\left(\frac{d W(t)}{d t}\right)_{0 A}=\left(\frac{d W(t)}{d t^{\prime}}\right)_{0}\left(\frac{d t^{\prime}}{d t}\right)_{0}=\frac{A}{0.99955}
$$

where the subscript $O A$ refers to the formulation above $0{ }^{\circ} \mathrm{C}$.

By subtracting eq (20) from eq (14), the difference in the derivatives at $0{ }^{\circ} \mathrm{C}$ of the two formulations becomes

$$
\begin{aligned}
\left(\frac{d W(t)}{d t}\right)_{0 B}-\left(\frac{d W(t)}{d t}\right)_{0 A} & =\Delta\left(\frac{d W(t)}{d t}\right)_{0}=\frac{1}{A_{1}} \\
& +\alpha-\alpha^{*}-\frac{A}{0.99955}
\end{aligned}
$$

From the relations

$$
A=\alpha\left(1+\delta / 100^{\circ} \mathrm{C}\right)
$$

and

$$
B=-10^{-4} \alpha \delta^{\circ} \mathrm{C}^{-2}
$$

$A^{*}$ and $B^{*}$ are obtained by substituting $\alpha^{*}$ and $\delta^{*}$ for $\alpha$ and $\delta$, respectively. By substituting $A^{*}$ and $B^{*}$ in eq (15), differentiating, and combining with eq (16), there results

$$
\left(\frac{d W^{*}(t)}{d t}\right)_{0 A}=\frac{A^{*}}{0.99955},
$$

an expression that is very similar to eq $(20)$. When eq (24) is compared with eq (8) and because the two derivatives are equal,

$$
\frac{1}{A_{1}}=\frac{A^{*}}{0.99955} \text {. }
$$

By substituting eq (25) for $1 / A_{1}$ in eq (21), there results

$$
\Delta\left(\frac{d W(t)}{d t}\right)_{0}=\alpha-\alpha^{*}+\frac{A^{*}-A}{0.99955} .
$$

Although $\Delta\left(\frac{d W(t)}{d t}\right)_{0}$ can be evaluated from eq (26), the conversion of $A^{*}$ and $A$ in terms of $B^{*}$ and $B$ results in an equation that is simpler to evaluate. The substitution of the expression (22) for $A^{*}$ and $A$ and then of the expression (23) for the $\alpha \delta$ product results in

$$
\Delta\left(\frac{d W(t)}{d t}\right)_{0}=\frac{0.00045\left(\alpha^{*}-\alpha\right)-100\left(B^{*}-B\right)}{0.99955} .
$$

The $\alpha$ values of SPRT's calibrated at NBS in the past few years range between 3.925 and about $3.927 \times 10^{-3}{ }^{\circ} \mathrm{C}^{-1}$ and the $B$ between -5.872 and $-5.877 \times 10^{-7}{ }^{\circ} \mathrm{C}^{-2}$; both $\alpha^{*}$ and $B^{*}$ are about the average of the above limits. Therefore, $\alpha^{*}-\alpha$ is about $\pm 1 \times 10^{-6}{ }^{\circ} \mathrm{C}^{-1}$ and $B-B^{*}$ is about $\pm 2.5 \times$ $10^{-10}{ }^{\circ} \mathrm{C}^{-2}$. (Note that " $100^{\circ}$ " in eq (27) has the unit ${ }^{\circ} \mathrm{C}$ associated with it.) When these values are substituted in eq (27)

$$
\Delta\left(\frac{d W(t)}{d t}\right)_{0} \sim 3 \times 10^{-8}{ }^{\circ} \mathrm{C}^{-1} .
$$

This may be taken as the maximum expected discontinuity. Any variation of this discontinuity is mostly dependent on the constant $B$. Inasmuch as $(d W(\mathrm{t}) / d t)_{0}$ is about $4 \times 10^{-3}{ }^{\circ} \mathrm{C}^{-1}$, the relative discontinuity in the first derivatives of the two formulations at $0^{\circ} \mathrm{C}$ is expected to be less than $8 \times 10^{-6}$. The discontinuity is negligible for most purposes. 


\section{APPEndix G. Derivation of Differential Coefficients for the Analysis of Errors in Platinum Resistance Thermometry}

This appendix deals with the propagation of errors in the temperature determination that results from the errors of calibration of a SPRT. The differential coefficients (the rate of change of the values of temperature with respect to the change of the resistance ratios, $R(t) / R(0)$, measured for calibration) are derived for the calibration measurements at the steam point, tin point, zinc point, and oxygen normal boiling point (NBP).

The total error, in the value of temperature that is obtained from measurements employing a SPRT, is the sum of the error introduced by the calibration measurements and the error from the experimenter's own measurements. The experimenter must determine his own observational error from a careful evaluation of his measurement techniques. He must also know the errors that may be introduced in the values of temperature caused by possible errors in the thermometer calibration measurements at the NBS. He must also be aware of possible errors from an unknown change in the calibration.

The variation among the SPRT's is yet another source of uncertainty; i.e., even though the measurements with the SPRT's are made at the same temperature (hotness), they do not yield exactly the same values of temperature. A number of scattered data do exist $[1,2,3,4]$, but there are no systematic high-precision measurements on the intercomparison of SPRT's that employ modern SPRT's and measuring equipment.

The errors of calibration at NBS may be separated into two types: (1) errors of deviation from realizing the fixed points and (2) errors of resistance measurements. The total differential of $t$ for the SPRT is given by

$$
d t=\sum\left(\frac{\partial t}{\partial t_{i}}\right) d t_{i}+\left(\frac{\partial t}{\partial W_{i}}\right) d W_{i},
$$

where $t_{i}=$ temperature of the defining fixed points, i.e., for this discussion the oxygen NBP, the triple point of water, the steam point or the tin point, and the zinc point. The $W_{i}$ refers to the measured resistance ratios $R\left(t_{i}\right) / R(0)$ corresponding to the fixed points. The $d t_{i}$ or $\delta t_{i}$ refers to a variation in the fixed-point temperature (hotness) experimentally realized, e.g., the deviation of the temperature of a tin-point cell. The differential coefficient $\left(\frac{\partial t}{\partial t_{i}}\right)$ represents the rate of change in the value of temperature with respect to a change in the $i$ th fixed-point temperature (hotness); the coefficients may be derived from the prescribed interpolation formulae using the values of defining fixed temperatures.
The differential coefficients are temperature dependent, i.e., $\left(\frac{\partial t}{\partial t_{i}}\right)=f(t)$.

The $d W_{i}$ or $\delta W_{i}$ refers to a variation in the resistance ratio measurement, e.g., the error of the resistance ratio measurement process with the SPRT at NBS. The differential coefficient $\left(\frac{\partial t}{\partial W_{i}}\right)$ represents the rate of change in the value of temperature with respect to a change in the measured resistance ratio at the $i$ th fixed point; the coefficients are derived from the prescribed formulae that relate the measured resistance ratio to the constants of the SPRT. Similar to $\left(\frac{\partial t}{\partial t_{i}}\right),\left(\frac{\partial t}{\partial W_{i}}\right)$ is also temperature dependent.

Some preliminary and limited investigations at NBS on the realization of the tin point and the triple point of water indicate that these fixed points are reproducible within $\pm 0.1 \mathrm{mK}$. This value, although obtained employing the same thermometer over a short interval of a few days to determine the error attributable to the variations in the temperatures of the fixed-point cells, includes both the variations in the temperature $\left(\delta t_{i}\right)$ of many freezes in different cells and the variations in the measurement process $\left(\delta W_{i}\right)$. Over a period of several months the reproducibility of measurements on different SPRT's employing the same cell is estimated to be about $\pm 0.2 \mathrm{mK}$. This value likewise includes any variations in the temperature of the cell as well as the variations in the measurement process. Thus, the errors of the realization of the fixed-point temperatures and the errors of calibration resistance measurements are difficult to separate. Therefore, the analysis of error propagation has been simplified by combining the two sources of errors in calibration measurements. Equation (1) then simplifies to

$$
d t=\sum\left(\frac{\partial t}{\partial W_{i}}\right) d W_{i}
$$

In the expression the change or error $\delta t_{i}$ in the value of temperature has been replaced by a corresponding change or error $\delta W_{i}$. At present, the total uncertainty (which includes $\delta t_{i}$ and $\delta W_{i}$ of eq (1)) of the calibration measurements is estimated to be 2 or $3 \mathrm{mK}$ at the oxygen NBP, and $1 \mathrm{mK}$ at the tin point, and $1 \mathrm{mK}$ at the zinc point.

The differential coefficients $\left(\frac{\partial t}{\partial W_{i}}\right)$ have been derived separately for two ranges of temperature 
defined by the SPRT, the range 0 to $630.74{ }^{\circ} \mathrm{C}$ and the range $-182.962(90.188 \mathrm{~K})$ to $0^{\circ} \mathrm{C}(273.15 \mathrm{~K})$. In the first range $\left(0\right.$ to $\left.630.74{ }^{\circ} \mathrm{C}\right)$, the coefficients have been derived for two types of calibration measurements: (1) at the triple point of water, the steam point, and the zinc point and (2) at the triple point of water, the tin point, and the zinc point. In the second range, the differential coefficients have also been derived for two types of calibration measurements: (1) at the oxygen NBP, the triple point of water, and the steam point and (2) at the oxygen NBP, the triple point of water, the tin point, and the zinc point.

The error $\delta t$ in the value of temperature, that would arise from an error $\delta W_{i}$ corresponding to a positive unit temperature (hotness), was calculated as a function of the temperature employing the derived differential coefficients; i.e., the function

$$
\delta t=\left(\frac{\partial t}{\partial W_{i}}\right) \delta W_{i}
$$

was evaluated as a function of the temperature. When the calibration error at a particular fixed point was considered, the calibration measurements at the other fixed points were taken to be correct. The following cases have been calculated and plotted in figure 17.

(1) The range $0{ }^{\circ} \mathrm{C}$ to $630.74{ }^{\circ} \mathrm{C}$

(a) calibration measurements made at the triple point of water, the steam point, and the zinc point; calibration error occurring only at the steam point or only at the zinc point,

(b) calibration measurements made at the triple point of water, the tin point, and the zinc point; calibration error occurring only at the tin point or only at the zinc point.

(2) The range $-182.962{ }^{\circ} \mathrm{C}(90.188 \mathrm{~K})$ to $0^{\circ} \mathrm{C}(273.15 \mathrm{~K})$

(a) calibration measurements made at the oxygen NBP, the triple point of water, and steam point; calibration error occurring only at the oxygen NBP or only at the steam point,

(b) calibration measurements made at the oxygen NBP, the triple point of water, the tin point, and the zinc point; calibration error occurring only at the oxygen NBP, or only at the tin point, or only at the zinc point.

In the range -182.962 to $0^{\circ} \mathrm{C}$, the calibration errors at the oxygen NBP for the cases $2 a$ and $2 b$ yield the same temperature error $\delta t$; therefore, the analysis of only one of these cases is given.

As a separate analysis, the case of an error in the realization of the triple point of water by the experimenter has been calculated; the calibration of the SPRT at NBS was considered perfect and an error of resistance measurement $\delta R$ corresponding to a positive unit temperature at the triple point of water was considered to have been made by the experimenter. The error $\delta R$ in the triple point of water measurements was taken to be propagated in the determination of the resistance ratio $R(t) / R(0)$. The results of the analysis are also plotted in figure 17.

The following sections deal with the details of the derivation of the differential coefficients that were employed to avaluate the temperature error function given by eq (3) for the cases cited in the previous paragraphs. In the discussion, the variations in the measurements at the fixed points will be described in terms of the resistance ratio, $W\left(t_{i}\right)=R\left(t_{i}\right) / R(0)$, the ratio of observed resistance at the fixed-point temperature $t_{i}$ to that at $0{ }^{\circ} \mathrm{C}$. The variations in $W\left(t_{i}\right)$ will be taken to be centered in $R\left(t_{i}\right)$. Obviously, any error in $R(0)$ will be reflected in $W\left(t_{i}\right)$; thus, the present discussion is also applicable to possible errors in the determination of $R(0)$.

1. $0^{\circ} \mathrm{C}$ to $630.74{ }^{\circ} \mathrm{C}$

From 0 to $630.74{ }^{\circ} \mathrm{C}$, the temperature $t$ on the IPTS -68 is defined by

$$
t=t^{\prime}+0.045\left(\frac{t^{\prime}}{s}\right)\left(\frac{t^{\prime}}{s}-1\right)\left(\frac{t^{\prime}}{z}-1\right)\left(\frac{t^{\prime}}{a}-1\right){ }^{\circ} \mathrm{C}
$$

and

$$
t^{\prime}=\frac{1}{\alpha}\left(W\left(t^{\prime}\right)-1\right)+\delta\left(\frac{t^{\prime}}{s}\right)\left(\frac{t^{\prime}}{s}-1\right){ }^{\circ} \mathrm{C},
$$

where

$$
W\left(t_{i}^{\prime}\right)=W\left(t_{i}\right)=R\left(t_{i}\right) / R(0)
$$

$s=100^{\circ} \mathrm{C}, z=419.58^{\circ} \mathrm{C}, a=630.74^{\circ} \mathrm{C}$, and $t=t_{68}$ (for convenience). The second term on the right side of eq (4) will, henceforth, be defined as

$$
M\left(t^{\prime}\right)=0.045\left(\frac{t^{\prime}}{s}\right)\left(\frac{t^{\prime}}{s}-1\right)\left(\frac{t^{\prime}}{z}-1\right)\left(\frac{t^{\prime}}{a}-1\right){ }^{\circ} \mathrm{C} .
$$

The function $M\left(t^{\prime}\right)$ represents a correction to $t^{\prime}$ to obtain the temperature $t$ that is a closer approximation to the thermodynamic scale. The values $t_{i}^{\prime}$ and $t_{i}$, therefore, represent the same temperature (hotness) and $R\left(t_{i}^{\prime}\right)=R\left(t_{i}\right)$. Also, eq (5) is equivalent to

$$
W\left(t^{\prime}\right)=1+A t^{\prime}+B t^{\prime 2}
$$

where

$$
A=\alpha\left(1+\delta / 100^{\circ} \mathrm{C}\right)
$$

and

$$
B=-10^{-4} \alpha \delta^{\circ} \mathrm{C}^{-2}
$$

The constants of the IPTS -68 eqs (5) and (8) are determined by measurement of the SPRT resistance at the triple point of water, the steam point, and the zinc point or the triple point of water, the tin point, 
and the zinc point. To simplify the symbols and terminology, the following definitions are made:

$$
\omega\left(t^{\prime}\right)=W\left(t^{\prime}\right)-1=A t^{\prime}+B t^{\prime 2},
$$

i.e.,

$$
\begin{gathered}
\omega_{S}=W_{S}-1=[R(100) / R(0)]-1 \\
\omega_{T}=W_{T}-1=[R(231.9292) / R(0)]-1,
\end{gathered}
$$

and

$$
\omega_{Z}=W_{Z}-1=[R(419.58) / R(0)]-1 .
$$

The differential coefficients will be derived first for the SPRT formulation with calibration measurements at the triple point of water, the tin point, and the zinc point. The differential coefficients sought are

$$
\left(\frac{\partial t}{\partial \omega_{T}}\right) \text { and }\left(\frac{\partial t}{\partial \omega_{Z}}\right)
$$

Because $\omega$ is a function of $t^{\prime}$, the conversion

$$
\frac{\partial t}{\partial \omega}=\left(\frac{\partial t}{\partial t^{\prime}}\right)\left(\frac{\partial t^{\prime}}{\partial \omega}\right)
$$

must be made. From eq (4),

$$
\begin{aligned}
\frac{\partial t}{\partial t^{\prime}}= & 1+\frac{0.045}{s^{2} z a}\left[4 t^{\prime 3}-3(s+z+a) t^{\prime 2}\right. \\
& \left.+2(s z+s a+z a) t^{\prime}-s z a\right] \\
= & 1+\frac{d M\left(t^{\prime}\right)}{d t^{\prime}}
\end{aligned}
$$

Equation (16) will be employed to convert $\partial t^{\prime} / \partial \omega$ to $\partial t / \partial \omega$.

From eq (11),

$$
\omega_{T}=A t_{T}^{\prime}+B t_{T}^{\prime 2}
$$

and

$$
\omega_{Z}=A t_{Z}^{\prime}+B t_{Z}^{\prime 2},
$$

where $t_{T}^{\prime}$ is the defined tin point temperature and $t_{Z}^{\prime}$ is the defined zinc point temperature. (According to the definition of the IPTS-68, $t_{T}^{\prime}=t_{68}-0.038937$ and $t_{z}^{\prime}=t_{68_{z}}$; henceforth, the "prime" symbol will not be employed in the designation of a fixed-point temperature.) Solving for $A$ and $B$, there results

$$
A=\left(\omega_{T} t_{Z}^{2}-\omega_{Z} t_{T}^{2}\right) / t_{T} t_{Z}\left(t_{Z}-t_{T}\right),
$$

and

$$
B=\left(\omega_{Z} t_{T}-\omega_{T} t_{Z}\right) / t_{T} t_{Z}\left(t_{Z}-t_{T}\right)
$$

Substituting in eq (11) for $A$ and $B$, there is obtained

$$
\omega\left(t^{\prime}\right)=\left[\frac{\left(\omega_{T} t_{Z}^{2}-\omega_{Z} t_{T}^{2}\right)}{t_{T} t_{Z}\left(t_{Z}-t_{T}\right)}\right] t^{\prime}+\left[\frac{\left(\omega \dot{Z} t_{T}-\omega_{T} t_{Z}\right)}{t_{T} t_{Z}\left(t_{Z}-t_{T}\right)}\right] t^{\prime 2} .
$$

The differential coefficients $\left(\frac{\partial t^{\prime}}{\partial \omega_{T}}\right)$ and $\left(\frac{\partial t^{\prime}}{\partial \omega_{z}}\right)$ are obtained by implicit differentiation of eq (21); for $\omega_{T}$, eq (21) yields

$$
\begin{aligned}
& \frac{t_{Z}^{2} t^{\prime}-t_{Z} t^{\prime 2}}{t_{T} t_{Z}\left(t_{Z}-t_{T}\right)} d \omega_{T} \\
& +\frac{\left[\left(\omega_{T} t_{Z}^{2}-\omega_{Z} t_{T}^{2}\right)+2\left(\omega_{Z} t_{T}-\omega_{T} t_{Z}\right) t^{\prime}\right]}{t_{T} t_{Z}\left(t_{Z}-t_{T}\right)} d t^{\prime}=0
\end{aligned}
$$

After transferring, eq (22) becomes

$$
\frac{\partial t^{\prime}}{\partial \omega_{T}}=\frac{t_{Z} t^{\prime}\left(t_{Z}-t^{\prime}\right)}{\omega_{T} t_{Z}\left(2 t^{\prime}-t_{Z}\right)-\omega_{Z} t_{T}\left(2 t^{\prime}-t_{T}\right)} .
$$

Similarly to obtain $\frac{\partial t^{\prime}}{\partial \omega z}$, the differentiation of eq (21) yields

$$
\begin{aligned}
& \frac{t_{T} t^{\prime 2}-t_{T}^{2} t^{\prime}}{t_{T} t_{Z}\left(t_{Z}-t_{T}\right)} d \omega_{Z} \\
& \quad+\frac{\left[\left(\omega_{T} t_{Z}^{2}-\omega_{Z} t_{T}^{2}\right)+2\left(\omega_{Z} t_{T}-\omega_{T} t_{Z}\right) t^{\prime}\right]}{t_{T} t_{Z}\left(t_{Z}-t_{T}\right)} d t^{\prime}=0 .
\end{aligned}
$$

After transferring, eq (24) becomes

$$
\frac{\partial t^{\prime}}{\partial \omega_{Z}}=\frac{t_{T} t^{\prime}\left(t_{T}-t^{\prime}\right)}{\omega_{Z} t_{T}\left(2 t^{\prime}-t_{T}\right)-\omega_{T} t_{Z}\left(2 t^{\prime}-t_{Z}\right)} .
$$

The desired differential coefficients $\frac{\partial t}{\partial \omega_{T}}$ and $\frac{\partial t}{\partial \omega_{Z}}$ are obtained by combining eqs (23) and (25), respectively, according to eq (15), with eq (16); there results, respectively,

$$
\frac{\partial t}{\partial \omega_{T}}=\left[1+\frac{d M\left(t^{\prime}\right)}{d t^{\prime}}\right] \frac{t_{Z} t^{\prime}\left(t_{Z}-t^{\prime}\right)}{\left[\omega_{T} t_{Z}\left(2 t^{\prime}-t_{Z}\right)-\omega_{Z} t_{T}\left(2 t^{\prime}-t_{T}\right)\right]},
$$

and

$$
\frac{\partial t}{\partial \omega_{Z}}=\left[1+\frac{d M\left(t^{\prime}\right)}{d t^{\prime}}\right] \frac{t_{T} t^{\prime}\left(t_{T}-t^{\prime}\right)}{\left[\omega_{Z} t_{T}\left(2 t^{\prime}-t_{T}\right)-\omega_{T} t_{Z}\left(2 t^{\prime}-t_{Z}\right)\right]^{3}}
$$

Equations (26) and (27) represent the rate of change of temperature $t$ with respect to a change in $\omega_{T}$ and $\omega_{z}$, respectively, at the temperature $t^{\prime}$. Although 
both equations (26) and (27) can be converted to terms of $t$ by employing the relation given by eq (4), the formulations given are simpler and practical to use. After calculating $\partial t / \partial \omega_{T}$ or $\partial t / \partial \omega_{Z}$ at $t^{\prime}$, then $t^{\prime}$ can be converted to $t$ according to eq (4).

The error in the temperature $t$ that results from the error in the tin point calibration is

$$
\delta t=\left(\frac{\partial t}{\partial \omega_{T}}\right)_{t^{\prime}} \delta \omega_{T}
$$

the error from the zinc point is

$$
\delta t=\left(\frac{\partial t}{\partial \omega_{Z}}\right)_{t^{\prime}} \delta \omega_{Z}
$$

Figure 17 shows the error that the experimenter would encounter for errors of $\delta \omega_{T}$ or $\delta \omega_{Z}$ corresponding to a unit positive error of temperature (hotness). The temperature in $t^{\prime}$ has been converted to $t$ in the figure.

The derivation of the differential coefficients for the SPRT formulation with calibration measurements at the triple point of water, steam point, and zinc point follow the same procedures outlined above. The final equations may be obtained directly by substituting $\omega_{S}$ for $\omega_{T}$ and $t_{S}$ for $t_{T}$ in eqs (26) and (27); the results are

$$
\frac{\partial t}{\partial \omega_{S}}=\left[1+\frac{d M\left(t^{\prime}\right)}{d t^{\prime}}\right] \frac{t_{Z} t^{\prime}\left(t_{Z}-t^{\prime}\right)}{\left[\omega_{S} t_{Z}\left(2 t^{\prime}-t_{Z}\right)-\omega_{Z} t_{S}\left(2 t^{\prime}-t_{S}\right)\right]}
$$

and

$\frac{\partial t}{\partial \omega_{Z}}=\left[1+\frac{d M\left(t^{\prime}\right)}{d t^{\prime}}\right] \frac{t_{S} t^{\prime}\left(t_{S}-t^{\prime}\right)}{\left[\omega_{Z} t_{S}\left(2 t^{\prime}-t_{S}\right)-\omega_{S} t_{Z}\left(2 t^{\prime}-t_{Z}\right)\right]}$.

The errors in the temperature $t$ that result from the errors in the calibration at the steam point and the zinc point are given by

$$
\delta t=\left(\frac{\partial t}{\partial \omega_{S}}\right)_{t} \delta \omega_{S}
$$

and

$$
\delta t=\left(\frac{\partial t}{\partial \omega_{Z}}\right)_{t^{\prime}} \delta \omega_{Z}
$$

Figure 17 shows the error that the experimenter would encounter for errors of $\delta \omega_{S}$ and $\delta \omega_{Z}$ corresponding to a unit positive error of temperature (hotness).

2. $90.188 \mathrm{~K}\left(-182.962{ }^{\circ} \mathrm{C}\right)$ to $273.15 \mathrm{~K}\left(0^{\circ} \mathrm{C}\right)$

From -182.962 to $0^{\circ} \mathrm{C}$ the temperature $t$ on the
IPTS $-68[5]$ is defined by the relation

$$
W(t)=W^{*}(t)+\Delta W(t),
$$

where

$$
W(t)=R(t) / R(0),
$$

and $W^{*}(t)$ is the resistance ratio given by the reference function in Celsius

$$
t=\sum_{i=1}^{20} A_{i}\left[\ln W^{*}(t)\right]^{i} .
$$

From eq (36)

$$
\delta t=\left(\partial t / \partial W^{*}(t)\right) \delta W^{*}(t),
$$

and from eq (34)

$$
\delta W^{*}(t)=-\delta \Delta W(t) .
$$

For the derivative $\partial t / \partial W^{*}(t)$, see Appendix E. The experimenter must determine his observational error $\delta W t)$. The deviation $\Delta W(t)$ is represented (in this temperature range and at $100{ }^{\circ} \mathrm{C}$ ) by the function

$$
\Delta W(t)=A_{4} t+C_{4} t^{3}\left(t-100^{\circ} \mathrm{C}\right),
$$

and the constants of the deviation equation are determined from the calibration measurements of $\Delta W(t)$ at the oxygen NBP, triple point of water, and the steam point. The deviation at $100{ }^{\circ} \mathrm{C}$, $\Delta W(100)$, may also be obtained from the calculated $W(100)$ based on the calibration of the SPRT at the triple point of water, the tin point, and the zinc point. The differential coefficients will be derived for both cases.

For convenience, the symbols and terminologies used in the text of the IPTS- 68 have been abbreviated as follows:

$$
\begin{aligned}
t & =t_{68}, \\
W^{*}(t) & =W_{\mathrm{CCT}-68}\left(t_{68}\right), \\
W(t) & =W\left(t_{68}\right),
\end{aligned}
$$

and

$$
\Delta W(t)=\Delta W\left(t_{68}\right) .
$$

In addition, the temperature of the fixed points will be indicated as:

$$
\begin{aligned}
& t_{0}=\text { oxygen NBP, } \\
& t_{S}=\text { steam point, } \\
& t_{T}=\text { tin point }\left(t_{T}=t_{68_{T}}-0.038937^{\circ} \mathrm{C}\right),
\end{aligned}
$$


and

$$
t_{z}=\text { zinc point }
$$

and the deviations $\Delta W(t)$ at the fixed points will be indicated as

$$
\Delta W_{0}=W_{0}-W_{0}^{*}(\text { deviation at the oxygen NBP) }
$$

$\Delta W_{S}=W_{S}-W_{S}^{*}$ (deviation at the steam point).

When the deviations at the oxygen NBP and the steam point are applied to eq (37), there results

$$
\begin{aligned}
& \Delta W_{0}=A_{4} t_{0}+C_{4} t_{0}^{3}\left(t_{0}-100\right) \\
& \Delta W_{S}=A_{4} t_{S}+C_{4} t_{S}^{3}\left(t_{S}-100\right) .
\end{aligned}
$$

(For simplification the ${ }^{\circ} \mathrm{C}$ unit after 100 and 75 will be deleted in some of the equations from 44 through 51.)

The expressions for $A_{4}$ and $C_{4}$ become

$$
A_{4}=\frac{\Delta W_{S} t_{0}^{3}\left(t_{0}-100\right)-\Delta W_{0} t_{S}^{3}\left(t_{S}-100\right)}{t_{S} t_{0}^{3}\left(t_{0}-100\right)-t_{0} t_{S}^{3}\left(t_{S}-100\right)}
$$

and

$$
C_{4}=\frac{\Delta W_{0} t_{S}-\Delta W_{S} t_{0}}{t_{S} t_{0}^{3}\left(t_{0}-100\right)-t_{0} t_{S}^{3}\left(t_{S}-100\right)}
$$

The differential coefficients $\left(\frac{\partial \Delta W(t)}{\partial \Delta W_{0}}\right)$ and $\left(\frac{\partial \Delta W(t)}{\partial \Delta W_{S}}\right)$ are obtained by differentiation after combining eqs (46) and (47) with eq (37); there results

$$
\frac{\partial \Delta W(t)}{\partial \Delta W_{0}}=\frac{-t t_{s}^{3}\left(t_{s}-100\right)+t_{s} t^{3}(t-100)}{t_{s} t_{0}^{3}\left(t_{0}-100\right)-t_{0} t_{s}^{3}\left(t_{s}-100\right)} .
$$

When $t_{s}=100{ }^{\circ} \mathrm{C}$ is substituted in expression (48), there is obtained

$$
\frac{\partial \Delta W(t)}{\partial \Delta W_{0}}=\frac{t^{3}(t-100)}{t_{0}^{3}\left(t_{0}-100\right)}
$$

For $\partial \Delta W(t) / \partial \Delta W_{S}$,

$\frac{\partial \Delta W(t)}{\partial \Delta W_{s}}=\frac{t t_{0}^{3}\left(t_{0}-100\right)-t_{0} t^{3}(t-100)}{t_{s} t_{0}^{3}\left(t_{0}-100\right)-t_{0} t_{s}^{3}\left(t_{s}-100\right)}$

and, when $t_{S}=100{ }^{\circ} \mathrm{C}$ is substituted wherever simplification can be made,

$$
\frac{\partial \Delta W(t)}{\partial \Delta W_{s}}=\frac{t\left[t_{0}^{2}\left(t_{0}-100\right)-t^{2}(t-100)\right]}{t_{s} t_{0}^{2}\left(t_{0}-100\right)}
$$

Figure 17 shows the error the experimenter would encounter for calibration errors of $\delta \Delta W_{0}$ or $\delta \Delta W_{S}$ corresponding to a unit positive error of temperature.

If the tin and zinc point calibrations are used, eqs (46) and (47) must be modified. Expressing $W_{S}$ and $W_{S}^{*}$ of eq (43) in the form of eq (8), there is obtained

where

$$
\Delta W_{S}=\left(A-A^{*}\right) t_{S}+\left(B-B^{*}\right) t_{S}^{2},
$$

and

$$
A^{*}=\alpha^{*}\left(1+\delta^{*} / 100^{\circ} \mathrm{C}\right)
$$

$$
B^{*}=10^{-4} \alpha^{*} \delta^{* \circ} \mathrm{C}^{-2},
$$

where $\alpha^{*}\left(3.9259668 \times 10^{-3} /{ }^{\circ} \mathrm{C}\right)$ and $\delta^{*}(1.496334$ ${ }^{\circ} \mathrm{C}$ ) are the constants of the IPTS-68 reference function above $0{ }^{\circ} \mathrm{C}$. The constants $A$ and $B$ are determined from calibration measurements at the tin and zinc points. By introducing the expressions for $A$ and $B$ from eqs (19) and (20) in eq (52), there results

$$
\begin{aligned}
\Delta W_{S}=\left[\frac{\omega_{T} t_{Z}^{2}-\omega_{Z} t_{T}^{2}}{t_{T} t_{Z}\left(t_{Z}-t_{T}\right)}-A^{*}\right] t_{S} \\
+\left[\frac{\omega_{Z} t_{T}-\omega_{T} t_{Z}}{t_{T} t_{Z}\left(t_{Z}-t_{T}\right)}-B^{*}\right] t_{S}^{2} .
\end{aligned}
$$

Equation (55) is then substituted for $\Delta W_{S}$ in eqs (46) and (47). The eq (49) for the differential coefficient of $\partial \Delta W(t) / \partial \Delta W_{0}$ remains unchanged. Because the true value of $\Delta W_{S}$ is independent of whether the calibration is at the steam point or at the tin and zinc points, the error function $\left(\partial \Delta W(t) / \partial \Delta W_{0}\right) \delta \Delta W_{0}$ for the case $2 b$ will be the same as that for case $2 a$ with the steam-point calibration.

Instead of substituting eq (55) in eqs (46) and (47) and performing a long algelraic process, the total derivative of $\Delta W_{S}$ can be obtained in terms of $\omega_{T}$ and $\omega_{z}$, i.e.,

$$
d \Delta W_{S}=\left(\frac{\partial \Delta W_{S}}{\partial \omega_{T}}\right) d \omega_{T}+\left(\frac{\partial \Delta W_{S}}{\partial \omega_{Z}}\right) d \omega_{Z}
$$

The expression (56) is employed to obtain $\delta \Delta W_{S}$, i.e.,

$$
\delta \Delta W_{S}=\left(\frac{\partial \Delta W_{S}}{\partial \omega_{T}}\right) \delta \omega_{T}+\left(\frac{\partial \Delta W_{S}}{\partial \omega_{T}}\right) \delta \omega_{Z}
$$

and the error function $\left(\frac{\partial \Delta W(t)}{\partial \Delta W_{S}}\right) \delta \Delta W_{S}$ is then evaluated as

$$
\begin{aligned}
\partial \Delta W(t)=\frac{\partial \Delta W(t)}{\partial \Delta W_{S}}\left[\left(\frac{\partial \Delta W_{S}}{\partial \omega_{T}}\right)\right. & \delta \omega_{T} \\
& \left.+\left(\frac{\partial \Delta W_{S}}{\partial \omega_{Z}}\right) \delta \omega_{Z}\right] .
\end{aligned}
$$


The coefficient $\frac{\partial \Delta W(t)}{\partial \Delta W_{S}}$ is given by eq (51) with eq (55) substituted for $\Delta W_{S}$. The differential coefficients $\frac{\partial \Delta W_{S}}{\partial \omega_{T}}$ and $\frac{\partial \Delta W_{S}}{\partial \omega_{Z}}$ are obtained from eq (55). Thus,

$$
\frac{\partial \Delta W_{S}}{\partial \omega_{T}}=\frac{t_{Z}^{2} t_{S}}{t_{T} t_{Z}\left(t_{Z}-t_{T}\right)}-\frac{t_{Z} t_{S}^{2}}{t_{T} t_{Z}\left(t_{Z}-t_{T}\right)}
$$

which simplifies to

$$
\frac{\partial \Delta W_{S}}{\partial \omega_{T}}=\frac{t_{S}\left(t_{Z}-t_{S}\right)}{t_{T}\left(t_{Z}-t_{T}\right)}
$$

For $\frac{\partial \Delta W_{S}}{\partial \omega_{Z}}$, eq $(55)$ becomes

$$
\frac{\partial \Delta W_{S}}{\partial \omega_{Z}}=\frac{-t_{T}^{2} t_{S}}{t_{T} t_{Z}\left(t_{Z}-t_{T}\right)}+\frac{t_{T} t_{S}^{2}}{t_{T} t_{Z}\left(t_{Z}-t_{T}\right)}
$$

which simplifies to

$$
\frac{\partial \Delta W_{S}}{\partial \omega_{Z}}=\frac{t_{S}\left(t_{S}-t_{T}\right)}{t_{Z}\left(t_{Z}-t_{T}\right)}
$$

Combining eqs (60) and (62) with eq (58), there is obtained

$$
\begin{aligned}
& \partial \Delta W(t)=\frac{\partial \Delta W(t)}{\partial \Delta W_{S}}\left[\frac{t_{S}\left(t_{Z}-t_{S}\right)}{t_{T}\left(t_{Z}-t_{T}\right)} \delta \omega_{T}\right. \\
& \left.+\frac{t_{S}\left(t_{S}-t_{T}\right)}{t_{Z}\left(t_{Z}-t_{T}\right)} \delta \omega_{Z}\right] .
\end{aligned}
$$

Figure 17 shows the error the experimenter would encounter for errors of $\delta \omega_{T}$ or $\delta \omega_{Z}$ corresponding to a positive unit error of temperature.

\section{References to Appendix G}

[1] Belyansky, L. B., Orlova, M. P., Sharevskaya, D. I., and Astrov, D. N., Investigation of the Resistance-Temperature Properties of Platinum for Resistance Thermometry over the Range from $90 \mathrm{~K}$ to $273 \mathrm{~K}$, Metrologia 5, No. 4, $107-111(1969)$.

[2] Hoge, H. J. and Brickwedde, F. G., Intercomparison of Platinum Resistance Thermometers Between $-190^{\circ}$ and $445^{\circ}$ C. J. Res. Nat. Bur. Stand. 28, 217-240 (February 1942) RP1454.

[3] Moussa, M. R. M., Van Dijk, H., Durieux, M., Comparison of Platinum Resistance Thermometers Between $63 \mathrm{~K}$ and $373.15 \mathrm{~K}$, Physica 40, 33-48 (1968).

[4] Sharevskaya, D. I., Orlova, M. P., Belyansky, L. B., and Galoushkina, G. A., Investigation of the ResistanceTemperature Properties of Platinum of Resistance Thermometry over the Range From $14 \mathrm{~K}$ to $90 \mathrm{~K}$, Metrologia 5 , No. 4, 103-107 (1969).

[5] The International Practical Temperature Scale of 1968. Adopted by the Comité International des Poids et Mesures,

- Metrologia 5, No. 2, 35-44 (April 1969).

\section{APPENDIX H. Calibration of Mueller Bridges}

\section{The Mueller Bridge}

The Mueller bridge is designed for measurement of temperatures in conjunction with four-terminal standard platinum resistance thermometers (SPRT). This bridge is basically the equal arm Wheatstone bridge but with important modifications. One of these modifications permits the SPRT connections to be commutated so that the lead resistances of the SPRT can be canceled by averaging the two bridge readings (see sec. 3.3). Other modifications include a provision for adjusting the ratio arms to equality and for reducing the uncertainties due to variations in contact resistance. In the process of determining the thermometer resistance the bridge current is reversed so that the effect of any steady spurious emf's are eliminated. The current reversal method also doubles the apparent null detector sensitivity.

The Mueller bridges employed in the Platinum Resistance Thermometer Laboratory of the NBS have additional features including the simultaneous interchange of the ratio arms when the SPRT connections are commutated. Any errors arising from a small inequality of the ratio arms are thus made negligible when the two bridge readings are averaged. The schematic circuit diagram of this form of Mueller bridge is shown in figure $\mathrm{H}-1$. For this discussion the upper two bridge arms will be referred to as the ratio arms, the lower left arm of the bridge plus the 0.1 and $1-\Omega$ decades as the rheostat arm, and the lower right arm as the thermometer or the SPRT arm of the bridge.

This bridge has seven twelve-position and one four-position measuring dial switches; the 0.00001 , $0.0001,0.001,0.01$, and $0.1-\Omega$ dials have positions $-1,0,1,2,3,4,5,6,7,8,9$, and $X(10)$ and the 1 and $10-\Omega$ dials have positions $0,1,2,3,4,5,6,7,8,9$, $X(10)$, and $Y(11)$. The $10-\Omega$ dial has the four positions $0,1,2$, and 3 . Thus, resistances between 0 and $422.1111 \Omega$ can be measured with the bridge. The 10 and $100-\Omega$ dial switches have mercury-wetted contacts; the $0.00001,0.0001,0.001$, and 0.01-dial switches, which have "dry" wiping contacts, have Waidner-Wolff resistance elements to reduce the effect of variations in the switch contact resistance. The 0.1 and $1-\Omega$ dial switches have dry wiping contacts which are in series with the relatively high resistance of the ratio-arm resistors to minimize the effect of variations in the contact resistance. The 


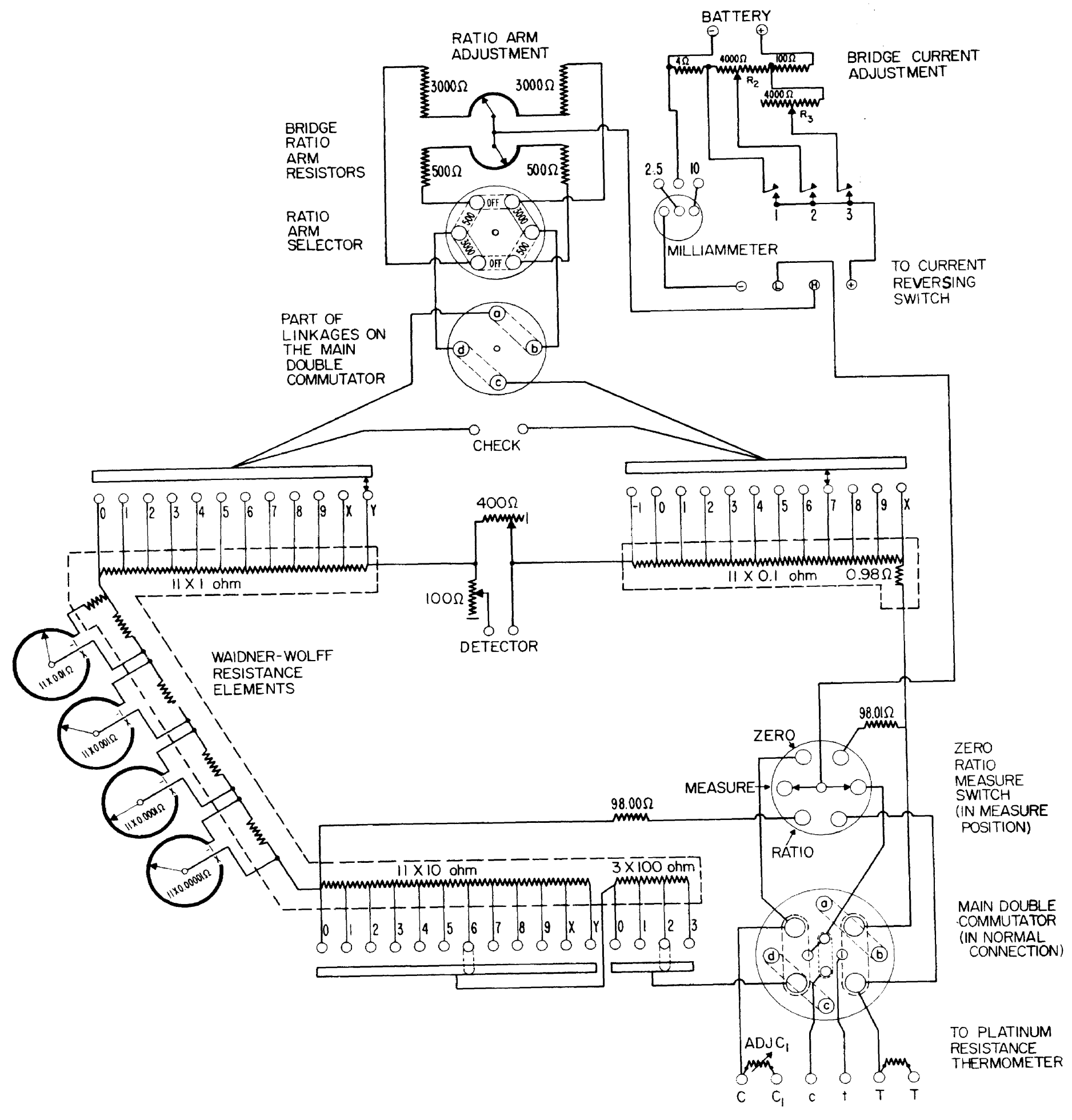

Figure H-1. Schematic circuitry of Mueller bridge employed in the Platinum Resistance Thermometry Laboratory at the National Bureau of Standards.

The resistors within the dashed lines are thermostated. 
$0.1-\Omega$ dial switch operates "subtractively". A mercury-wetted, three-position switch permits the selection of 500 or $3000-\Omega$ ratio arms or the "off" position. In the "off" position both ratio arm resistors are disconnected from the lower sections of the bridge. The operation of the bridge for adjustment of the ratio arms, for determination of the bridge zero, or for measurement of a SPRT resistance is selected by means of a three-position, ratio-zero-measure switch, which is a dry wiping contact switch for the older models of this Mueller bridge but a mercurywetted switch for the newer models. A mercurywetted switch (commutator switch) commutates the SPRT connection and simultaneously interchanges the ratio arms. The principal resistors of the bridge, shown enclosed in the shaded portion of figure $\mathrm{H}-1$ are thermostated at about $35^{\circ} \mathrm{C}$.

Terminals are provided for batteries and for a current reversing switch. A milliammeter located in the battery circuit indicates the current through the SPRT (one-half of the bridge current). Variable resistors are incorporated in the bridge for adjusting the current. Terminals and variable resistors for damping are provided for the null detector. Special terminals and an adjustable resistor permit the equalization of the potential lead resistances of the SPRT.

\section{Auxiliary Equipment for Calibrating Mueller Bridges}

To calibrate the Mueller bridge, the following special equipment (other than null detector, current reversing switch, and batteries that are necessary in regular resistance measurements) is required:

(1) Adjustable decade resistance box that is suff. ciently stable during the time required to intercompare two combinations of resistances (dial settings) of the Mueller bridge. At NBS a second Mueller bridge serves as this auxiliary adjustable resistor. (To avoid confusion the auxiliary Mueller bridge will, henceforth, be referred to as the auxiliary adjustable resistor.)

(2) Standard resistor of $10 \Omega$ (preferably) or $100 \Omega$ with a recent calibration to convert the bridge resistance into terms of the national resistance unit. Duplicate resistors will help reduce the calibration uncertainties.

(3) A thermometer and an insulated enclosure or thermostatted oil bath for the standard resistors.

\section{Preliminary Preparation}

\subsection{Bridge Thermostat}

The bridge resistors should have been thermostatted at a constant temperature at least one week prior to calibration. Safety provisions should be made to avoid any large deviation from the thermostatted temperature; e.g., auxiliary control and battery power should be provided to maintain the bridge temperature whenever the main power line is disrupted. The bridge should be recalibrated periodically and whenever the temperature control fails.

\subsection{Switches}

The old mercury should be removed from the mercury-wetted switches and the studs and links amalgamated with clean mercury. The studs of the dry wiping contact switches should be cleaned and lubricated with pure petroleum. The spaces between the wiping switch blades should be freed of accumulated dirt.

\subsection{Bridge Ratio Arms}

Either the ratio arm resistors of 500 or $3000 \Omega$ may be selected; the equality of the ratio arms is adjusted separately by means of the slide-wire resistors which have the resistance range of about 0.02 percent of that of the ratio arm resistors. The "tap" (contact) on the slide wire resistor is in the battery circuit so that any variations in the contact resistance has no effect on the bridge balance. The choice of either 500 - or $3000-\Omega$ ratio arms is based upon the resistance of the SPRT to be determined, the voltage supply available, and the insulation resistance between the voltage supply and the bridge components. (One is particularly concerned with leakage between the voltage supply and the null detector or thermometer.) The contact and lead resistances associated with the 0.1 - and $1-\Omega$ dials is in series with the ratio arm resistors. For a given variation in the contact resistance of these switches, the uncertainty of a bridge reading due to these switches and their leads is greater for the $500-\Omega$ ratio arm resistors than for the $3000 \Omega$ resistors. Problems with insulation leakage, on the other hand, increase with both the value of the voltage supply and the resistance of the ratio arms.

To adjust the ratio arms to equality, the threeposition, ratio-zero-measure dial switch is placed in the "ratio" position. In this position, two auxiliary resistors (each of about $98 \Omega$ ) are each connected to the rheostat and SPRT arms of the bridge; also, the 10- and $100-\Omega$ resistors are "by-passed." The SPRT should be disconnected. The "tap" of the slide-wire resistor is initially placed near the midpoint. The bridge is balanced with the ratio arms in one position and balanced again with the ratio arms interchanged. The bridge dials are then set on the average of the two balance readings and the slide wire adjusted to balance the bridge. The setting on the slide-wire resistor should be checked by balancing the bridge again with the two positions of the ratio arms. The ratio arms have been adjusted to equality when the two bridge readings are the same. (All bridge balance readings, unless stated otherwise, are performed with current reversal.) 


\subsection{Bridge Zero}

A residual resistance exists in the rheostat arm even with all measuring switches set on zero because of the resistances of the leads that connect the resistor net work and the resistances of the switch contacts. The Waidner-Wolff elements contribute also to the residual resistance. Therefore, in the bridge design a resistor (about $0.98 \Omega$ ) is placed in series with the $0.1-\Omega$ decade resistors to balance the rheostat and SPRT arms of the bridge when all dials are set to zero. The remaining deviation from a perfect balance is the "bridge zero." The bridge zero must be subtracted algebraically from the dial readings of all measurements.

To determine the bridge zero, the bridge ratio arms are first equalized (see sec. 3.3 ) and the threeposition, ratio-zero-measure switch is then placed on the "zero" position. The bridge zero is the average of the bridge balance readings obtained with the commutator switch in normal $(N)$ and reverse $(R)$ positions. If the contact resistance of the three-position selector switch varies between the $N$ and $R$ readings, the bridge zero will be in error. If the selector switch is considered unreliable, which it may be if a dry wiping contact switch is used for a selector switch, the bridge zero is determined by connecting a special shorting link made by soldering three copper wires (about AWG 18) into the form of an " $\mathrm{H}$." The " $\mathrm{H}$ " should be about $5 \mathrm{~cm}$ high and $10 \mathrm{~cm}$ wide. One short vertical wire of the " $\mathrm{H}$ " is connected between the terminals $C$ and $T$; the other short vertical wire of the " $H$ " is connected between the terminals $c$ and $t$. When using this special shorting link to determine the bridge zero the three-position selector switch should be in the "measure" position. The bridge zero is the average of the bridge balance readings obtained with the commutator in normal and reverse positions.

\subsection{Resistance of Leads Between the 1-and 0.I- $\Omega$ Decade Resistors and the Ratio Arm Switch}

The resistors of the $0.1-$ and 1- $\Omega$ step decades are enclosed, along with other principal resistors, in a thermostatted aluminum block. The resistances of the leads from these resistors to the dial switches must be sufficiently close so that their differences have negligible effect on the ratio of the ratio arms. The double commutator that interchanges the ratio arm resistors does not interchange the resistances of the leads from the resistors of the 0.1 - and $1-\Omega$ step decades, the switch contact resistances of these decades, nor the resistances of the leads from the dial switches of these decades to the ratio arm reversing switch (see bridge circuit diagram, fig. 4-1).

The design of the bridge permits the separate determination of the degree of equality of the leads from the 0.1 - and $1-\Omega$ decade resistors to their dial switches and of the leads from these switches to the ratio arm reversing switch. The "check" terminals are employed to test the degree of equality of the resistor lead plus the switch contact resistances; the resistances of the leads between the dial switches and the ratio arm reversing switch is determined by measurements using another bridge. By introducing branch points at locations a and $c$ of the ratio-arm reversing switch, the degree of equality of the combined leads may be determined (see fig. H-2). The discussion to follow in this section (3.5) will assume that a pair of wires from points $a$ and $c$ are available at special terminals on the bridge. If the special wiring is not available, an equivalent circuit may be achieved by removing the double commutator and placing amalgamated heavy copper links from a to $b$ and $c$ to $d$. Wires are attached to the links close to the a and c studs of the switch and are then connected to a doublepole, double-throw (DPDT) all copper knife switch as shown schematically in figure $\mathrm{H}-2$. Amalgamated links for the SPRT and battery are also placed on the double commutator studs corresponding to resistance measurement in the $\mathrm{N}$ connection (see fig. $\mathrm{H}-1$ ). If the special wiring and terminals are available on the bridge, the double commutator is placed in the $\mathrm{N}$ position. The null detector is connected to the Mueller bridge through the DPDT switch so that the switch can be connected to the

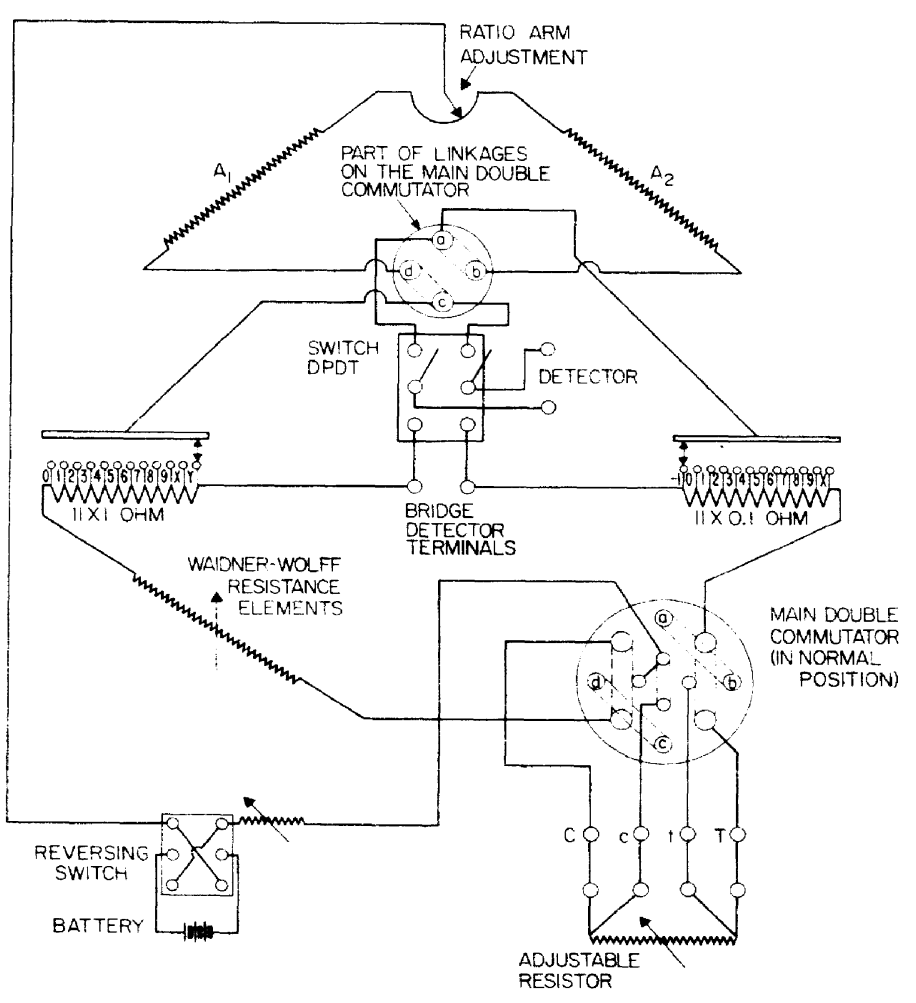

FIGURE H-2. Schematic circuitry for testing the equality of the Mueller bridge leads between the ratio arm reversing switch (part of the main commutator switch) and $0.1-\Omega$ and $1-\Omega$ decades.

The contact resistances of the decade switches are included as part of the bridge leads. 
leads from positions $a$ and $c$ of the ratio-arm reversing switch or to the bridge galvanometer posts (see figs. $\mathrm{H}-1$ and $\mathrm{H}-2$ ). The ratio-zero-measure dial switch is placed on the "measure" position and the auxiliary adjustable resistor is connected as a four-terminal resistor to the SPRT posts. (At NBS the auxiliary Mueller bridge is prepared for this and subsequent bridge calibration applications by shorting the "check" terminals and by placing its commutator in the normal position, the galvanometer damping resistors in the open positions, the ratio-zero-measure switch in the "measure" position, and the ratio arm selector switch in the "off" position. Three heavy gauge copper wires are connected from the $C$ and $T$ posts and the shorted "check" terminals of the auxiliary bridge to the $T$, C, and c posts, respectively, of the bridge to be calibrated. In the above circuit, when the dials of the auxiliary Mueller bridge are set to zero, approximately equal resistances are introduced into the lower two arms of the Mueller bridge to be calibrated; the $0.1-\Omega$ decade operates subtractively (see fig. $\mathrm{H}-1)$ ).

Instead of testing all possible combinations of the switch positions of the $0.1-$ and $1-\Omega$ decades, the maximum difference between the lead resistances is determined by comparing the 0 step of the $0.1-\Omega$ decade with all steps of the $1-\Omega$ decade and 0 step of the $1-\Omega$ decade with all steps of the
$0.1-\Omega$ decade, totaling 22 comparisons. With 0.1 and $1-\Omega$ decade dials on 0 and the DPDT switch thrown to the $a-c$ position, the bridge is balanced (reading 1) employing the lower dials and the auxiliary adjustable resistor. The DPDT switch is then thrown to the galvanometer post position and the bridge is balanced (reading 2) using the lower dials only. The difference in the balance readings is, for all practical purposes, the difference in the lead resistance. The process is repeated with the $1-\Omega$ decade on the 1 position. A sample of the complete test data is summarized in table 1 . (The tabular values given are only for descriptive purposes and do not represent any actual data.) The maximum error in the bridge reading is, in proportional parts, $\frac{74}{500} \times 10^{-6}$ or $\frac{74}{3000} \times 10^{-6}$, depending upon the choice of ratio arm resistors. For this particular (fictitious) bridge the results demonstrate that in the determination of high resistances the $3000-\Omega$ ratio arms should be employed, also that significantly large error may occur in the determination of small resistance differences in which the $0.1-$ and $1-\Omega$ decade switches are moved.

After determining the differences in the lead resistances the auxiliary connections are removed and the Mueller bridge is placed in the normal operating condition.

TABLE 1. Comparison and determination of the maximum difference in the lead resistance between the 0.1-and 1 -ohm decades and the ratio arm reversing switch

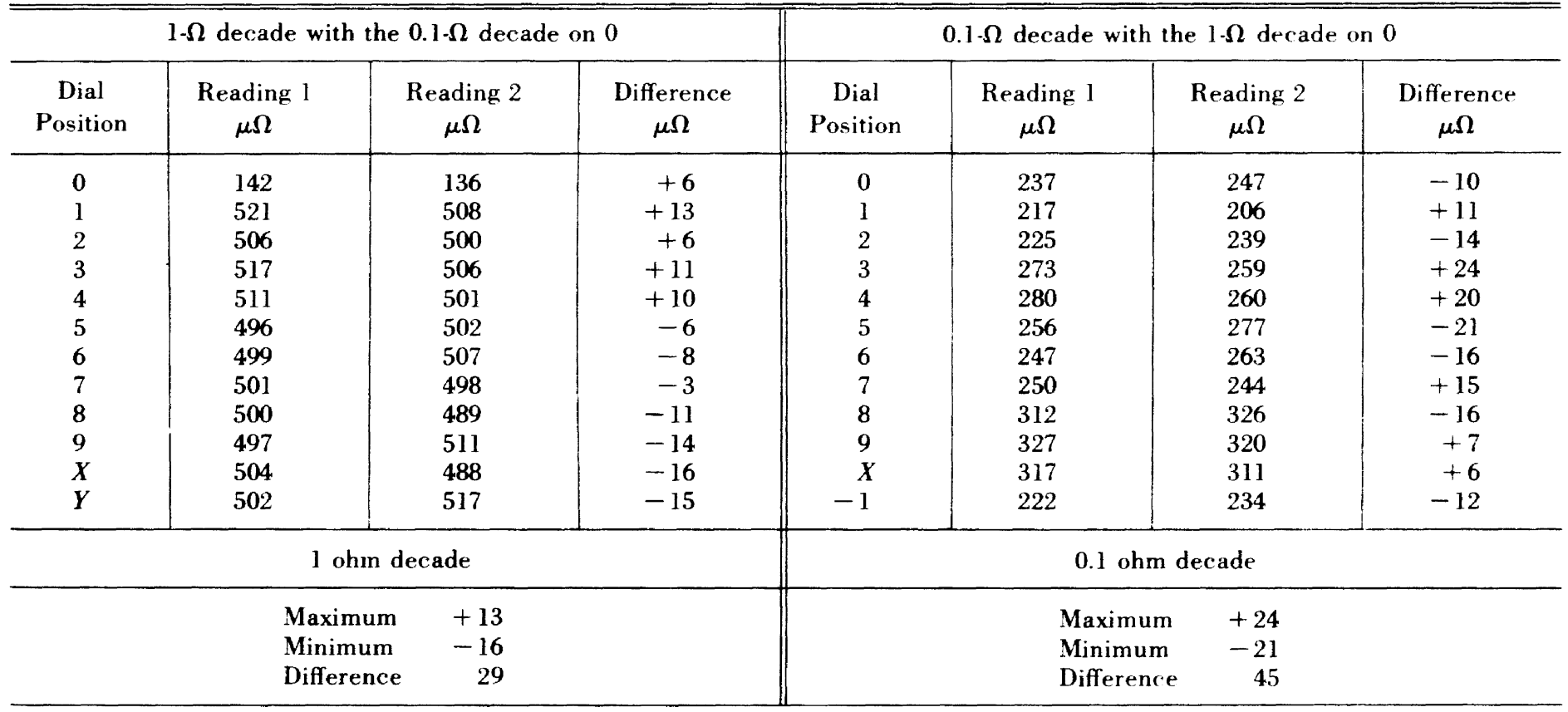

Sum of difference $=74$

Error $=$ Bridge Reading $\times \frac{74}{500} \times 10^{-6} \Omega$ or Bridge Reading $\times \frac{74}{3000} \times 10^{-6} \Omega$ 


\subsection{Calibration of the Mueller Bridge in Terms of the National Unit of Resistance}

To calibrate the Mueller bridge in terms of the national unit of resistance the $X$ position of the $10-\Omega$ decade and/or the $X$ position of the $1-\Omega$ decade are compared with recently calibrated $100-\Omega$ and/or $10-\Omega$ standard resistors, respectively. The choice of the comparison depends upon the stability of the standard resistors and the bridge decades that are most used in the measurements. On the basis of absolute resistance, resistors of higher resistance are usually less stable. In the NBS Platinum Resistance Thermometry Laboratory the Mueller bridge is usually compared with $10-\Omega$ standard resistors. (The bridge resistors may all be expressed in terms of, for example, the resistance of $0.1_{x}$ being exactly $l$ bridge unit and, if the SPRT resistance ratio $R(t) / R(0)$ is employed in all temperature evaluations, the bridge unit is satisfactory. The resistance in terms of the national unit is, however, necessary for some comparisons, e.g., comparison of the measured $R\left(0^{\circ} \mathrm{C}\right)$ of the SPRT with that obtained at the National Bureau of Standards.)

The $X$ position of the $1-\Omega$ decade is compared with a $10-\Omega$ standard resistor by measuring the standard, as a four-terminal resistor, directly with the Mueller bridge. The following table 2 gives an example of the results obtained. (One or two figures more than are experimentally realized are carried in the calculations for internal consistency.)

TABLE 2. Comparison of $X$ position of $I-\Omega$ dial with a 10- $\Omega$ standard resistor

(Standard resistor at $26.4^{\circ} \mathrm{C}$ )

\begin{tabular}{|c|c|}
\hline & Ohms \\
\hline $\begin{array}{l}\text { Standard at } 25^{\circ} \mathrm{C} \ldots \ldots \ldots \ldots \ldots \ldots \ldots \ldots \\
\text { Temperature correction to } 26.4{ }^{\circ} \mathrm{C} \ldots\end{array}$ & $\begin{array}{c}10.00026_{14} \\
-0.0000047\end{array}$ \\
\hline Standard at $26.4^{\circ} \mathrm{C} \ldots \ldots \ldots \ldots$ & 10.0002567 \\
\hline $\begin{array}{l}\text { Bridge reading, average of } \mathbf{N} \text { and } \mathbf{R} \\
\text { readings } \ldots \ldots \ldots \ldots \ldots \ldots \ldots \ldots \ldots \ldots \ldots \ldots \ldots \ldots \ldots \ldots \ldots \ldots \ldots \ldots \ldots \ldots \ldots \ldots \ldots\end{array}$ & $\begin{array}{r}X .0010491 \\
0.0001832\end{array}$ \\
\hline $\begin{array}{c}\text { Bridge } \text { reading corrected for bridge } \\
\text { zero } \ldots \ldots \ldots \ldots \ldots \ldots \ldots \ldots \ldots \ldots \ldots \ldots \ldots \ldots \ldots \ldots \ldots \ldots \ldots\end{array}$ & X.0008659 \\
\hline $\begin{array}{l}\text { Standard resistor minus bridge reading } \\
\text { corrected for bridge zero } \ldots \ldots \ldots \ldots \ldots \ldots \ldots \ldots \ldots \ldots \ldots \ldots \ldots \ldots \ldots \ldots \ldots \ldots\end{array}$ & $\begin{array}{c}-0.0006092 \\
10-0.0006092 \Omega\end{array}$ \\
\hline
\end{tabular}

\section{Calibration of the Mueller Bridge Decade Steps}

The ratio arms of the Mueller bridge must be adjusted to equality before calibrating the 0.1 or
$1-\Omega$ decade steps. The double commutator is then placed in the "normal" position and the auxiliary adjustable resistor is connected as a four terminal resistor to the SPRT posts. As previously stated, all bridge balance readings are obtained with current reversal.

In principle the resistance steps of the Mueller bridge are calibrated internally by comparing each step of one decade with the $X$ position of the next lower decade. The differences between the resistors can be obtained conveniently in terms of the lower decades. If upon analyzing the calibration data from the higher resistance decade down to the lower resistance decades the latter decades should show significant corrections, the differences expressed in terms of them could then be corrected and the calibration data reanalyzed (see step (18)).

The procedure used at the NBS Thermometry Laboratory for calibrating the Mueller bridge is as follows:

(1) Set to 1 on the $1-\Omega$ decade of the bridge and to 0 on the $0.1-\Omega$ decade and use the lower decades to balance an appropriate resistance in the auxiliary adjustable resistor. Galvanometer (detector) deflections are interpolated to obtain fractions of the $0.00001-\Omega$ step.

(2) Without disturbing the external circuit, change the bridge setting to 0 on the $1-\Omega$ decade and to $X$ on the $0.1-\Omega$ decade and again use the lower decades to balance the bridge. The dials of the auxiliary adjustable resistor should be adjusted so that the lower decades are near zero for the lesser of (1) or (2) bridge balances.

(3) Repeat (2) and then (1).

(4) Set to 2 on the $1-\Omega$ decade of the bridge and to 0 on the $0.1-\Omega$ decade and balance with a corresponding resistance in the auxiliary adjustable resistor, as in (1).

(5) Change the bridge setting to 1 on the $1-\Omega$ decade and to $X$ on the $0.1-\Omega$ decade and proceed as in (2) and (3).

(6) Continue this process until each of the $1-\Omega$ steps has been compared with the $X$ position of the $0.1-\Omega$ decade.

(7) The observations under (1), (2), and (3) yield the relation:

$$
1_{1}+a_{1}=0.1_{x}+b_{1}
$$

where the Arabic numeral index indicates reference to the resistance change due to that individual step in the decade, the Roman numeral index $X$ indicates reference to the total resistance change due to changing the decade switch from position 0 to position $X$ (or 10), and where $a_{1}$ and $b_{1}$ are the corrected averages of the respective lower decade readings, including galvanometer interpolations, which were obtained in the balancings of the bridge. The relation (1), when transferred to

$$
1_{1}-0.1_{\mathrm{X}}=b_{1}-a_{1}=c_{1},
$$


indicates the difference in resistance between step 1 of $1 \Omega$ decade and $X$ of the $0.1-\Omega$ decade.

Equations similar to (2) are obtained from (4), (5), and (6) leading to the remainder of the set:

$$
\begin{aligned}
& 1_{2}-0.1_{X}=c_{2} \\
& 1_{3}-0.1_{X}=c_{3} \\
& 1_{4}-0.1_{X}=c_{4} \\
& 1_{5}-0.1_{X}=c_{5} \\
& 1_{6}-0.1_{X}=c_{6} \\
& 1_{7}-0.1_{X}=c_{7} \\
& 1_{8}-0.1_{X}=c_{8} \\
& 1_{9}-0.1_{X}=c_{9} \\
& 1_{10}-0.1_{X}=c_{10} .
\end{aligned}
$$

Table 3 summarizes the results of such a comparison between the $1-\Omega$ decade and $0.1_{x}$. In the twodigit notation of the dial pairs that are given in column 1 , the first digit indicates the dial position of the decade being compared and the second digit the dial position of the next lower decade. The values listed in the second column correspond to the resistance differences, the $c$ 's, of eqs (2) through (3).

(8) Summing eqs (2) and (3), there is obtained

$$
\sum_{i=1}^{10} 1_{i}-10 \times 0.1_{\mathrm{x}}=\sum_{i=1}^{10} c_{i}
$$

but

$$
\sum_{i=1}^{10} 1_{i}=1 \mathrm{x}
$$

The comparison of $1_{x}$ with a $10-\Omega$ standard resistor showed that

$$
1_{\mathrm{X}}=10-0.0006092 \Omega \text {. }
$$

(See table 2, sec. 3.6). Combining eqs (4), (5), and (6),

$$
0.1_{\mathrm{X}}=\left(10-0.0006092-\sum_{i=1}^{10} c_{i}\right) / 10
$$

From table 3, $\sum_{i=1}^{10} c_{i}=-0.0003746 \Omega$ and, therefore, $0.1_{X}=1-0.00002346 \Omega$. The relation (7) is shown evaluated at the bottom of table 3 .

(9) Each step of column 2 (table 3 ) is corrected for the real value of $0.1_{x}$ according to eqs (2) and (3) in terms of the national resistance unit. Column 3 lists the corrected values.

(10) The stepwise cumulative corrections of the dial positions are listed in column 4 and rounded to the nearest $0.1 \mu \Omega$ in column 5 . The last entry of columns 4 and 5 must agree with the value of $l_{X}$ obtained by comparison with the standard resistor. This serves as a check on arithmetic operations.
TABLE 3. Calibration of $1-\Omega$ decade by comparison with $0.1_{X}$

\begin{tabular}{c|c|c|c|c}
\hline \hline $\begin{array}{c}\text { Dial } \\
\text { pairs } \\
\text { compared }\end{array}$ & $\begin{array}{c}\text { Differ- } \\
\text { ence } \\
0.1 \mu \Omega\end{array}$ & $\begin{array}{c}\text { Corrected } \\
0.1 \mu \Omega\end{array}$ & $\Sigma, 0.1 \mu \Omega$ & $\begin{array}{c}\text { Nearest } \\
0.1 \mu \Omega\end{array}$ \\
\hline $\mathbf{1 0 - 0 X}$ & -359 & -593.6 & -593.6 & -594 \\
$\mathbf{2 0 - 1 X}$ & -298 & -532.6 & -1126.2 & -1126 \\
$\mathbf{3 0 - 2 X}$ & -408 & -642.6 & -1768.8 & -1769 \\
$\mathbf{4 0 - 3 X}$ & -406 & -640.6 & -2409.4 & -2409 \\
$\mathbf{5 0 - 4 X}$ & -385 & -619.6 & -3029.0 & -3029 \\
$\mathbf{6 0 - 5 X}$ & -368 & -602.6 & -3631.6 & -3632 \\
$\mathbf{7 0 - 6 X}$ & -376 & -610.6 & -4242.2 & -4242 \\
$\mathbf{8 0 - 7 X}$ & -386 & -620.6 & -4862.8 & -4863 \\
$\mathbf{9 0 - 8 X}$ & -414 & -648.6 & -5511.4 & -5511 \\
$\mathbf{X 0 - 9 X}$ & -346 & -580.6 & -6092.0 & -6092 \\
\hline
\end{tabular}

$\Sigma$ differences $=-3746$

$0.1_{X}=(10-0.0006092-(-0.0003746)) / 10$

$=1-0.00002346 \Omega$.

(11) The $0.1-\Omega$ decade steps are compared with $0.0 l_{x}$ of the next lower dial following the procedures given in (1), (2), (3), (4), (5), and (6). A set of relations given by (2) and (3) is then obtained. Table 4 shows the results of a comparison.

(12) A relation similar to (4) is given by

$$
\sum_{i=1}^{10} 0.1_{i}-10 \times 0.01_{X}=\sum_{i=1}^{10} c_{i},
$$

where

$$
\sum_{i=1}^{10} c_{i}=-0.0000318 \Omega \text { and }
$$

$\sum_{i=1}^{10} 0.1_{i}=0.1_{X}=1-0.00002346 \Omega$

There results similar to relation (7)

$0.01_{X}=(1-0.00002346-(-0.0000318)) / 10$

$$
=0.1+0.00000083 \Omega \text {. }
$$

(13) Each dial step of column 2 (table 4) is corrected for the real value of $0.01_{X}$ in terms of the national resistance unit. Column 3 lists the corrected dial steps. Column 4 lists the stepwise cumulative corrections and column 5 lists values of column 4 rounded to the nearest $0.1 \mu \Omega$.

(14) The $0.01-\Omega$ decade steps are compared with $0^{0.001}{ }_{X}$ of the next lower decade following the same procedures given in (1), (2), (3), (4), (5) and (6). The measurements are analyzed according to (7), (8), (9), (10), (13) and (14). The results of such a comparison are summarized in table 5 . The $0.001-\Omega$ and $0.001-\Omega$ decade steps are calibrated in the same manner; the results are given in tables 6 and 7 , respectively. 
TABLE 4. Calibration of $0.1-\Omega$ decade by comparison with $0.01_{X}$

\begin{tabular}{c|c|c|c|c}
\hline $\begin{array}{c}\text { Dial } \\
\text { pairs } \\
\text { compared }\end{array}$ & $\begin{array}{c}\text { Differ- } \\
\text { ence } \\
0.1 \mu \Omega\end{array}$ & $\begin{array}{c}\text { Corrected } \\
0.1 \mu \Omega\end{array}$ & $\Sigma, 0.1 \mu \Omega$ & $\begin{array}{c}\text { Nearest } \\
0.1 \mu \Omega\end{array}$ \\
\hline $\mathbf{1 0 - 0 X}$ & -51 & -42.7 & -42.7 & -43 \\
$20-1 X$ & -07 & +1.3 & -41.4 & -41 \\
$\mathbf{3 0 - 2 X}$ & -17 & -8.7 & -50.1 & -50 \\
$\mathbf{4 0 - 3 X}$ & -42 & -33.7 & -83.8 & -84 \\
$\mathbf{5 0 - 4 X}$ & -59 & -50.7 & -134.5 & -134 \\
$\mathbf{6 0 - 5 X}$ & -42 & -33.7 & -168.2 & -168 \\
$\mathbf{7 0 - 6 X}$ & -28 & -19.7 & -187.9 & -188 \\
$\mathbf{8 0 - 7 X}$ & -34 & -25.7 & -213.6 & -214 \\
$\mathbf{9 0 - 8 X}$ & -28 & -19.7 & -233.3 & -233 \\
$\mathbf{X 0 - 9 X}$ & -10 & -1.7 & -235.0 & -235 \\
\hline
\end{tabular}

$\Sigma$ differences $=-318$

$0.01_{X}=(1-0.00002346-(-0.0000318)) / 10$

$=0.1+0.00000083 \Omega$.

TABLE 5. Calibration of $0.01-\Omega$ decade by comparison with $0.001_{x}$

\begin{tabular}{c|c|c|c|c}
\hline $\begin{array}{c}\text { Dial } \\
\text { pairs } \\
\text { compared }\end{array}$ & $\begin{array}{c}\text { Differ- } \\
\text { ence } \\
0.1 \mu \Omega\end{array}$ & $\begin{array}{c}\text { Corrected } \\
0.1 \mu \Omega\end{array}$ & $\Sigma, 0.1 \mu \Omega$ & $\begin{array}{c}\text { Nearest } \\
0.1 \mu \Omega\end{array}$ \\
\hline $\mathbf{1 0 - 0 X}$ & +7 & +16.8 & +16.8 & +17 \\
$\mathbf{2 0 - 1 X}$ & -19 & -9.2 & +7.6 & +8 \\
$\mathbf{3 0 - 2 X}$ & +13 & +22.8 & +30.4 & +30 \\
$\mathbf{4 0 - 3 X}$ & -19 & -9.2 & +21.2 & +21 \\
$\mathbf{5 0 - 4 X}$ & -30 & -20.2 & +1.0 & +1 \\
$\mathbf{6 0 - 5 X}$ & -4 & +5.8 & +6.8 & +7 \\
$\mathbf{7 0 - 6 X}$ & -5 & +4.8 & +11.6 & +12 \\
$\mathbf{8 0}-7 \mathrm{X}$ & +6 & +15.8 & +27.4 & +27 \\
$\mathbf{9 0 - 8 X}$ & -27 & -17.2 & +10.2 & +10 \\
$\mathbf{X 0 - 9 X}$ & -12 & -2.2 & +8.0 & +8 \\
\hline
\end{tabular}

$\Sigma$ differences $=-90$

$0.001_{X}=(0.1+0.00000083-(-0.0000090)) / 10$

$=0.01+0.00000098 \Omega$.

TABLE 6. Calibration of $0.001-\Omega$ decade by comparison with $0.0001_{X}$

\begin{tabular}{c|c|c|c|c}
\hline $\begin{array}{c}\text { Dial } \\
\text { pairs } \\
\text { compared }\end{array}$ & $\begin{array}{c}\text { Differ- } \\
\text { ence } \\
0.1 \mu \Omega\end{array}$ & $\begin{array}{c}\text { Corrected } \\
0.1 \mu \Omega\end{array}$ & $\Sigma, 0.1 \mu \Omega$ & $\begin{array}{c}\text { Nearest } \\
0.1 \mu \Omega\end{array}$ \\
\hline $10-0 \mathrm{X}$ & +2 & -0.4 & -0.4 & 0 \\
$20-1 \mathrm{X}$ & +4 & +1.6 & +1.2 & +1 \\
$30-2 \mathrm{X}$ & +2 & -0.4 & +0.8 & +1 \\
$40-3 \mathrm{X}$ & +4 & +1.6 & +2.4 & +2 \\
$\mathbf{5 0 - 4 X}$ & +1 & -1.4 & +1.0 & +1 \\
$\mathbf{6 0 - 5 X}$ & +4 & +1.6 & +2.6 & +3 \\
$70-6 \mathrm{X}$ & +3 & +0.6 & +3.2 & +3 \\
$\mathbf{8 0 - 7 X}$ & +4 & +1.6 & +4.8 & +5 \\
$90-8 X$ & +4 & +1.6 & +6.4 & +6 \\
$\mathbf{X 0 - 9 X}$ & +6 & +3.6 & +10.0 & +10 \\
\hline
\end{tabular}

$\Sigma$ differences $=+34$

$0.0001_{x}=(0.01+0.00000098-0.0000034) / 10$

$=0.001-0.00000024 \Omega$.
TABLE 7. Calibration of $0.0001-\Omega$ decade by comparison with $0.00001_{X}$

\begin{tabular}{c|c|c|c|c}
\hline \hline $\begin{array}{c}\text { Dial } \\
\text { pairs } \\
\text { compared }\end{array}$ & $\begin{array}{c}\text { Differ- } \\
\text { ence } \\
0.1 \mu \Omega\end{array}$ & $\begin{array}{c}\text { Corrected } \\
0.1 \mu \Omega\end{array}$ & $\Sigma, 0.1 \mu \Omega$ & $\begin{array}{c}\text { Nearest } \\
0.1 \mu \Omega\end{array}$ \\
\hline $\mathbf{1 0 - 0 X}$ & +1 & +0.5 & +0.5 & +0 \\
$\mathbf{2 0 - 1 X}$ & +1 & -1.5 & -1.0 & -1 \\
$\mathbf{3 0 - 2 X}$ & -1 & -1.5 & -2.5 & -2 \\
$\mathbf{4 0 - 3 X}$ & 0 & -0.5 & -3.0 & -3 \\
$\mathbf{5 0 - 4 X}$ & +2 & +1.5 & -1.5 & -2 \\
$\mathbf{6 0 - 5 X}$ & -1 & -1.5 & -3.0 & -3 \\
$\mathbf{7 0 - 6 X}$ & +1 & +0.5 & -2.5 & -2 \\
$\mathbf{8 0 - 7 X}$ & +1 & +0.5 & -2.0 & -2 \\
$\mathbf{9 0 - 8 X}$ & 0 & -0.5 & -2.5 & -2 \\
$\mathbf{X 0 - 9 X}$ & +1 & +0.5 & -2.0 & -2 \\
\hline
\end{tabular}

$\Sigma$ differences $=+3$

$0.00001_{x}=(0.001-0.00000024-0.0000003) / 10$ $=0.0001-0.00000005 \Omega$.

TABLE 8. Calibration of $0.00001 \Omega$ decade

\begin{tabular}{c|c|c|c|c}
\hline $\begin{array}{c}\text { Dial } \\
\text { pairs } \\
\text { compared }\end{array}$ & $\begin{array}{c}\text { Differ- } \\
\text { ence } \\
0.1 \mu \Omega\end{array}$ & $\begin{array}{c}\text { Corrected } \\
0.1 \mu \Omega\end{array}$ & $\Sigma, 0.1 \mu \Omega$ & $\begin{array}{c}\text { Nearest } \\
0.1 \mu \Omega\end{array}$ \\
\hline $\mathbf{1 0 - 0 X}$ & +2 & +1.6 & +1.6 & +2 \\
$20-1 \mathrm{X}$ & +1 & +0.6 & +2.2 & +2 \\
$\mathbf{3 0 - 2 X}$ & 0 & -0.4 & +1.8 & +2 \\
$\mathbf{4 0 - 3 X}$ & 0 & -0.4 & +1.4 & +1 \\
$\mathbf{5 0 - 4 X}$ & 0 & -0.4 & +1.0 & +1 \\
$\mathbf{6 0 - 5 X}$ & -2 & -2.4 & -1.4 & -1 \\
$\mathbf{7 0 - 6 X}$ & 0 & -0.4 & -1.8 & -2 \\
$\mathbf{8 0 - 7 X}$ & +2 & +1.6 & -0.2 & 0 \\
$\mathbf{9 0 - 8 X}$ & +1 & +0.6 & +0.4 & 0 \\
$\mathbf{X 0 - 9 X}$ & 0 & -0.4 & 0 & 0 \\
\hline
\end{tabular}

$\Sigma$ differences $=+4$

$0.000001_{x}=(0.0001-0.00000005-0.0000004) / 10$ $=0.00001-0.00000004 \Omega$.

(15) The $0.00001-\Omega$ decade is calibrated by the method of galvanometer deflection. Set the 0.00001$\boldsymbol{\Omega}$ dial on zero with the bridge nearly balanced by appropriately setting the auxiliary adjustable resistor. Step to position $\mathrm{X}$ and record the galvanometer deflection. Assume $1 / 10$ of the deflection to correspond to $0.00000 l_{X}$ which will be adjusted to the true value in the manner as the previous $X$ 's were adjusted. Set the $0.00001-\Omega$ dial back to zero position and step to the 1 position. Record the deflection difference with respect to the imaginary $0.000001_{X}$. Balance and repeat with other steps of the dial. The results of a sample data are summarized in table 8. The sum of the galvanometer deflections corresponds to $0.0001-0.00000005 \Omega$ (see the bottom of table 7 ). 
(16) The $10-\Omega$ decade is calibrated by comparison with the $X$ position of the 1- $\Omega$ decade following the procedures (1), (2), (3), (4), (5), (6), and (7). The results of the comparison are summarized in column 2 of table 9. The resistance of $l_{X}$ is 10-0.0006092 ohms (see table 2). Column 3 (table 9) lists the dial steps corrected to the national resistance units. Column 4 lists the stepwise cumulative corrections.

(17) The $100 \Omega$ dial steps are calibrated by comparison with $10_{x}$. Column 2 to table 10 summarizes the results. The resistance of $10_{x}=100-0.003717 \Omega$ (see table 9). The column 3 (table 10) lists the corrected dial steps and column 4 lists the stepwise cumulative corrections.

(18) All differences, i.e., c's of eq (2), in the dial readings should be examined to determine whether they are changed significantly when the dial readings are corrected according to the calibration. If the correction is significant, then a second analysis should be made employing the corrected differences.

(19) The decade steps of the sample data have been compared with the $X$ of the next lower decade in terms of the lower decade steps (see column 2 of tables 3 to 10 ). The decades from $0.00001-\Omega$ to $1-\Omega$ are compared in terms of the $0.00001-\Omega$ decade dial positions; the $10-\Omega$ decade and the three $100-\Omega$ steps are compared in terms of the 0.00001 - and $0.0001-\Omega$ steps. The $0.001-\Omega$ decade dial was used at one occasion with the $100-\Omega$ dials (see table 10 ). In as much as the lower dials have very small corrections, the bridge resistors including the auxiliary adjustable resistor should be adjusted so that the comparison measurements would involve changes in dial positions of the less significant dials wherever possible (see step (2)). By following this procedure the calibration correction to the differences ( $c$ 's of eq (2)) can be made small or negligible and a second analysis (step (18)) of the calibration data based on the corrected values of c's may be avoided. In the sample calibration data the corrections to the lower dials are in the range of interpolated values of the least significant dial $(0.00001-\Omega)$. The corrections to the differences (c's) are negligible.
(20) The results of calibration given in tables 3 to 10 are summarized in table 11 for rapid reference in making bridge dial corrections. Corrections of less than one $\mu \Omega$ may be taken as zero without significantly increasing the uncertainty of the resistance measurements.

(21) For further information reference may be made to the following:

(a) Brooks, P. P. B., Calibration procedures for direct-current resistance apparatus, Nat. Bur. Stand. (U.S.), Monogr. 39, 53 pages (March 1, 1962).

(b) Brooks, P. P. B., Calibration of Mueller Thermometer bridges, 39 pages (October 1965), from the National Bureau of Standards List of Publications LP38 (August 1966), Calibration Procedures of the NBS, Electrical Reference Standards Section.

TABLE 9. Calibration of 10- $\Omega$ decade by comparison with $l_{\boldsymbol{X}}$

\begin{tabular}{c|c|c|c}
\hline $\begin{array}{c}\text { Dial pairs } \\
\text { compared }\end{array}$ & $\begin{array}{c}\text { Difference } \\
\mu \Omega\end{array}$ & $\begin{array}{c}\text { Corrected } \\
\mu \Omega\end{array}$ & $\Sigma, \mu \Omega$ \\
\hline $10-0 \mathrm{X}$ & +289 & -320 & -320 \\
$20-1 \mathrm{X}$ & +227 & -382 & -702 \\
$30-2 \mathrm{X}$ & +287 & -322 & -1024 \\
$40-3 \mathrm{X}$ & +015 & -594 & -1618 \\
$50-4 \mathrm{X}$ & +167 & -442 & -2060 \\
$60-5 \mathrm{X}$ & -009 & -618 & -2678 \\
$70-6 \mathrm{X}$ & +272 & -337 & -3015 \\
$80-7 \mathrm{X}$ & +576 & -033 & -3048 \\
$90-8 \mathrm{X}$ & +267 & -342 & -3390 \\
$\mathrm{X0-9X}$ & +282 & -327 & -3717 \\
\hline
\end{tabular}

$1_{X}=10-0.0006092 \Omega$.

TABLE 10. Calibration of 100- $\Omega$ dial steps by comparison with $10_{X}$

\begin{tabular}{c|c|c|c}
\hline $\begin{array}{c}\text { Dial pairs } \\
\text { compared }\end{array}$ & $\begin{array}{c}\text { Difference } \\
\mu \Omega\end{array}$ & $\begin{array}{c}\text { Corrected } \\
\mu \Omega\end{array}$ & $\Sigma, \mu \Omega$ \\
\hline $10-0 \mathrm{X}$ & -945 & -4662 & -4662 \\
$20-1 \mathrm{X}$ & -1710 & -5427 & -10089 \\
$30-2 \mathrm{X}$ & +973 & -2744 & -12833 \\
\hline
\end{tabular}

$10_{x}=100-0.003717 \Omega$.

TABLE 11. Summary of Mueller bridge calibration Bridge corrections in micro-ohms

\begin{tabular}{l|c|c|c|c|c|c|c|c}
\hline \hline \multicolumn{1}{c}{ Dial } \\
$\begin{array}{l}\text { posi- } \\
\text { tion }\end{array}$ & 100 & 10 & 1 & 0.1 & 0.01 & 0.001 & 0.0001 & 0.00001 \\
\hline 1 & -4662 & -320 & -59.4 & -4.3 & +1.7 & 0 & 0 & +0.2 \\
2 & -10089 & -702 & -112.6 & -4.1 & +0.8 & +0.1 & -0.1 & +0.2 \\
3 & -12833 & -1024 & -176.9 & -5.0 & +3.0 & +0.1 & -0.2 & +0.2 \\
4 & & -1618 & -240.9 & -8.4 & +2.1 & +0.2 & -0.3 & +0.1 \\
5 & & -2060 & -302.9 & -13.4 & +0.1 & +0.1 & -0.2 & +0.1 \\
6 & & -2678 & -363.2 & -16.8 & +0.7 & +0.3 & -0.3 & -0.1 \\
7 & & -3015 & -424.2 & -18.8 & +1.2 & +0.3 & -0.2 & -0.2 \\
8 & & -3048 & -486.3 & -21.4 & +2.7 & +0.5 & -0.2 & 0 \\
9 & & -3390 & -551.1 & -23.3 & +1.0 & +0.6 & -0.2 & 0 \\
$\mathrm{X}$ & & -3717 & -609.2 & -23.5 & +0.8 & +1.0 & -0.2 & 0 \\
\hline
\end{tabular}




\section{APPENDIX I. Calibration of the Linearity of a Potentiometer}

The equipment required for calibrating the linearity of a potentiometer is:

(a) A source of adjustable stable voltage, e.g., a second potentiometer or an adjustable well regulated electronic voltage supply.

(b) A detector of sufficient sensitivity. (For the resolution of $1 \mu \Omega$ at $1 \mathrm{ma}$ in the SPRT a sensitivity of one nanovolt is needed.)

The calibration procedure, analogous to that of the Mueller bridge (see Appendix $\mathrm{H}$ ), is the comparison of successive voltage steps of one decade against the $X$ position of the next lower decade. The sensitivity of the detector should be sufficient to permit interpolation of the last dial of the potentiometer. The current reversal procedure will double the detector sensitivity and cancel any steady extraneous emf's.

\section{Calibration of the Decade Switches}

Let ${ }_{j} \Delta_{i}$ be the difference in nanovolts between the $i$ th step of decade $j$ and the $X$ position of $j+1$ (next lower) decade, i.e.,

$$
{ }_{j} \Delta_{i}=\left({ }_{j} V_{i}-{ }_{j} V_{i-1}\right)-{ }_{j+1} V_{X},
$$

where $V$ is the voltage associated with the dial position. (The decade $j=1$ is the most significant decade.) The average difference between each of the steps of decade $j$ and $X$ of decade $j+1$ is:

$$
{ }_{j} \bar{\Delta}=\frac{1}{10} \sum_{i=1}^{10}{ }_{j} \Delta_{i}=\left({ }_{j} V_{X}-10_{j+1} V_{X}\right) / 10 .
$$

The difference between each observed ${ }_{j} \Delta_{i}$ and ${ }_{j} \bar{\Delta}$ is

$$
\begin{aligned}
{ }_{j} \Delta_{i}-{ }_{j} \bar{\Delta}={ }_{j} \Delta_{i}-\frac{1}{10} \sum_{i=1}^{10}{ }_{j} \Delta_{i}={ }_{j} \delta_{i} \\
=\left({ }_{j} V_{i}-{ }_{j} V_{i-1}\right)-{ }_{j} V_{X} / 10 .
\end{aligned}
$$

Equation (3) shows that the calibration process reduces to the comparison of each step of the decade with the average step of the decade. The deviations from linearity ${ }_{j} D_{n}$ at the $n$th step of decade $j$ is the algebraic sum of the deviations from the average difference of the 1 st to $n$th steps inclusive, i.e.,

$$
\begin{gathered}
{ }_{j} D_{n}=\sum_{i=1}^{n}{ }_{j} \delta_{i} \\
=\sum_{i=1}^{n}\left[{ }_{j} \Delta_{i}-\frac{1}{10} \sum_{i=1}^{10}{ }_{j} \Delta_{i}\right]={ }_{j} V_{n}-n_{j} V_{X} / 10 .
\end{gathered}
$$

In eqs (4) and (5), ${ }_{j} D_{10} \equiv 0$. Table 1 shows an example of calibration data and calculations of linearity corrections on "decade 1 ," the most significant dial with steps nominally $0.01 \mathrm{~V}$, of a six-dial potentiometer. The record of the differences between dial positions and $X$ of the next lower decade is given in nanovolts, but the actual laboratory record is recorded as galvanometer deflections and the sensitivity.

The procedure for determining the linearity of the lower decades is the same as that for decade 1 , i.e., the dial positions of decade 2 are compared with the $X$ position of decade 3 and so forth. Tables of differences ${ }_{j} \Delta_{i}$, differences ${ }_{j} \delta_{i}$ from the mean ${ }_{j} \bar{\Delta}$, and the deviations from linearity ${ }_{j} D_{n}$ are constructed as shown in table 1 for decade 1 .

The linearity of the least significant decade is determined from galvanometer deflections. The galvanometer deflection corresponding to each step is compared with the average deflection for the decade (see eq (3)). Since the least significant decade can tolerate relatively large deviations in the resistance elements, usually its linearity is found to be adequate for most measurements.

The above calibration data yield the deviation from linearity of a dial position in terms of the average step of the decade (see eq (5)). The overall linearity of the measuring decades is determined in terms of the linearity of the dial positions of a decade and the relation of its $X$ position with the average step of the next higher decade (eq (2)), i.e., ${ }_{i} \bar{\Delta}$ or $6.7 \mathrm{nV}$ (see table 1 for the deviation of the average step of decade 1 from $X$ position of decade 2). The $X$ position of decade 2 is $6.7 \mathrm{nV}$ smaller than the average step of decade 1 ; each step or the average step of decade 2 is, therefore, $6.7 / 10 \mathrm{nV}$ smaller. The total deviation from linearity of the $n$th step of decade 2 is the sum of the deviations from linearity within the decade (as determined from comparison

TABLE 1. Example of calibration of potentiometer dial 1 steps = nominally $0.01 \mathrm{~V}$

\begin{tabular}{c|c|c|c}
\hline $\begin{array}{c}\text { Position } i \\
\text { dial I }\end{array}$ & $\begin{array}{c}{ }_{1} \Delta_{i} \\
\text { nanovolts }\end{array}$ & $\begin{array}{c}{ }_{1} \delta_{i} \\
\text { nanovolts }\end{array}$ & $\begin{array}{c}{ }_{1} D_{n} \\
\text { nanovolts }\end{array}$ \\
\hline 1 & 21 & 14.3 & 14 \\
2 & 33 & 26.3 & 41 \\
3 & -47 & -53.7 & -13 \\
4 & -52 & -58.7 & -72 \\
5 & 34 & 27.3 & -44 \\
6 & 43 & 36.3 & -8 \\
7 & 62 & 55.3 & 47 \\
8 & -11 & -17.7 & 29 \\
9 & 27 & 20.3 & 50 \\
$X$ & -43 & -49.7 & 0 \\
\hline
\end{tabular}

$\sum_{i=1}^{10}{ }_{1} \Delta_{i}=67 \mathrm{nV}$.

${ }_{1} \bar{\Delta}=6.7 \mathrm{nV}$. 
of the 2d decade steps with the $X$ position of the $3 d$ decade) and the deviation between decades 1 and 2 , i.e.,

$$
{ }_{2} \epsilon_{n}={ }_{2} D_{n}-n_{1} \bar{\Delta} / 10
$$

${ }_{2} \epsilon_{n}$ is the total deviation from linearity of the $n$th step of decade 2.

The total deviation from linearity of the $m$ th step of decade 3 is:

$$
{ }_{3} \epsilon_{m}={ }_{3} D_{m}-m\left[{ }_{2} \bar{\Delta} / 10+{ }_{1} \bar{\Delta} / 100\right] .
$$

The total deviation from linearity of the $k$ th step of decade 4 is:

$$
{ }_{4} \epsilon_{k}={ }_{4} D_{k}-k\left[{ }_{3} \bar{\Delta} / 10+{ }_{2} \bar{\Delta} / 100+{ }_{1} \bar{\Delta} / 1000\right] .
$$

For the $p$ th step of decade 5 the total deviation from linearity is:

$$
{ }_{5} \epsilon_{p}={ }_{5} D_{p}-p\left[{ }_{4} \bar{\Delta} / 10+{ }_{3} \bar{\Delta} / 100+{ }_{2} \bar{\Delta} / 1000+{ }_{1} \bar{\Delta} / 10000\right] \text {. }
$$

The term containing ${ }_{1} \bar{\Delta} / 10000$ or even ${ }_{2} \bar{\Delta} / 1000$ may be insignificant. The deviations from linearity of even lower decades are calculated as an extension of the above.

\section{Calibration of the Range Switches}

In contrast to the Mueller bridge, potentiometers have range switches with $x 1, x 0.1$, or even $x 0.01$ factors. Any deviations from the nominal factors are determined by comparing the $X$ position of decade 1 on the $x 0.1$ range and 1 position of decade 1 on the $x 1$ range corrected for linearity. The $x 0.01$ range is compared in the same manner with $x 0.1$ range.

\subsection{To Check the x0.1 Range Factor}

With the range switch set on $x 1$ position, adjust the external voltage source until it is balanced against the step 1 of the first $(j=1)$ decade. When a balance is achieved, change the range switch to $x 0.1$ position and the first decade to step $X$. Since the step 1 of the first decade is $1 \mathrm{ppm}$ too high (from the calibration given in table 1), the dial settings should be $X 00001$ on the six-dial potentiometer being employed in the example given here. This gives an accurate 10 to 1 ratio for the dial settings. If the potentiometer current with the range switch set on $x 0.1$ position is too high, then (using current reversals) the galvanometer deflections will have the same sense with the increase in the dial setting. If the current is too low, then the galvanometer deflection will have the opposite sense with the increase in the dial setting. If, e.g., galvanometer deflection is equivalent to +10 nanovolts, the range factor correction becomes $+1 \times 10^{-6}$.

\subsection{To check the $x 0.01$ Range Factor}

The $x 0.01$ range is compared with the $x 0.1$ range in the same manner as above between $x 0.1$ and $x 1$ ranges. The range factor correction for the $x 0.01$ range relative to the $x l$ range is then the combined correction factors (very nearly the sum of the correction factors) for the $x 0.1$ range and the $x 0.01$ range relative to the $x 0.1$ range.

\section{Combined Decade Switch and Range Switch Corrections}

The potentiometer voltage equation with calibration correction is:

$$
V=F(1+K)\left(E_{1}+E_{2}+E_{3}+E_{4}+E_{5}+E_{6}\right.
$$

where

$$
\left.+\epsilon_{1}+\epsilon_{3}+\epsilon_{3}+\epsilon_{4}+\epsilon_{5}+\epsilon_{6}\right)
$$

$F=$ range factor $x 1, x 0.1$, or $x 0.01$

$K=$ range factor correction for $x 0.1$ or $x 0.01$ ranges, zero for $x l$ range

$E_{1}$ through $E_{6}=$ nominal dial readings for the decades $\epsilon_{1}$ through $\epsilon_{6}=$ total deviations from linearity of the dial settings.

The above calibration procedure is suitable for a "simple" potentiometer with one working battery. When the battery current is branched (split-circuit), for example in the Wenner voltage-difference potentiometer [1], the ratio of the currents in the two branches must be precisely adjusted before comparing a decade in one branch with a decade in the other and checked periodically during this intercomparison.

Instruments with more than one potentiometer in tandem, for example the White potentiometer [4, $5,6]$ and other more recent designs [3], require precise standardization of the currents when the decades between two potentiometers are compared.

For application of universal ratio sets to potentiom. eter calibration see NBS Monograph 39 by Brooks [2].

\section{References to Appendix I}

[1] Behr, L., The Wenner potentiometer, Rev. Sci. Instr. 3, No. 3, 109-120 (1932).

[2] Brooks, P. P. B., Calibration procedures for direct-current resistance apparatus, Nat. Bur. Stand. (U.S.), Monogr. 39, 53 pages (March 1, 1962).

[3] Schaeffer, W. H., The six-dial thermofree potentiometer, Instruments and Control Systems 34, No. 2, 283-291 (February 1961).

[4] White, W. P., Potentiometers for thermoelectric measurements especially in calorimetry, J. Am. Chem. Soc. 36, 1868-1885 (1914).

[5] White, W. P., Einige neue Doppelkompensatoren. I. Thermokraftfreie Kontakte, mechanische Anordnungen und Zubehorteile, Zeit. Instrumentenk. 34, 71-82 (1914).

[6] White, W. P., Einige neue Doppelkompensatoren. II. Die elektrische Einrichtung, Zeit. Instrumentenk. 34, 107$113,142-151$ (1914). 


\section{A PPENDIX J. Guide for Obtaining the Calibration of Standard Platinum Re- sistance Thermometers at the National Bureau of Standards}

The following guides are intended for users and those immediately connected with the application of SPRT's who are interested in obtaining calibration services at the NBS or who are planning to purchase SPRT's.

The SPRT calibration equipment and procedures employed by NBS are for four-terminal thermometers that meet the IPTS -68 specification of quality (see sec. 2) and that are constructed within certain external physical dimensions and immersion requirements. To obtain calibration services within the regular time schedule, the SPRT's must conform to these specifications. Any deviations from these specifications will result in additional cost, time, or rejection from calibration, e.g. the calibration of an over-sized SPRT is prohibitively expensive.

\section{Inquiries and Purchase Orders for the SPRT Calibration Service}

1.1. Inquiries and purchase orders shall be directed to the attention of:

$$
\begin{aligned}
& \text { Temperature Section, Heat Division } \\
& \text { B04 Physics Building } \\
& \text { National Bureau of Standards } \\
& \text { Washington, D.C. } 20234
\end{aligned}
$$

1.2. Attention is called to paragraphs (d), (e), and (f) of Section 200.100 in NBS Special Publication 250, Calibration and Test Services of the National Bureau of Standards (1970 edition) which deal with shipping, insurance, and risk of loss or damage of instruments sent to NBS for calibration and test. These three paragraphs are quoted below for convenience of reference.

(d) Shipping and insurance coverage instructions should be clearly and legibly shown on the purchase order for the calibration or test. The customer must pay shipping charges to and from NBS; shipments from NBS will be made collect. The method of return transportation should be stated and it is recommended that return shipments be insured, since NBS will not assume liability for their loss or damage. For long-distance shipping it is found that air express and air freight provide an advantage in reduction of time in transit. If return shipment by parcel post is requested or is a suitable mode of transportation, shipments will be prepaid by NBS but without covering insurance. When no shipping or insurance instructions are furnished, return shipment will be made by common carrier collect and uninsured.

(e) NBS will not be responsible for the risk of loss or damage to any item during shipment to or from the Bureau. Any arrangements for insurance covering this risk must be made by the customer. Return shipment will be made by the Bureau as indicated in paragraph (d) of this Section. The purchase order should always show the value of the equipment and if transit insurance is carried by the customer this fact should be stated.

(f) The risk of loss or damage in handling or testing of any item by NBS must be assumed by the customer, except when it is determined by the Bureau that such loss or damage was occasioned solely by the negligence of bureau personnel.

1.3. The user of the SPRT shall assume the responsibility to provide the following information either directly or through his procurement group. If any one item of the requested information is lacking, the calibration will be delayed. To minimize the "paper work," all necessary information should be forwarded in a single communication.

1.3.1. Manufacturer, model number, and serial number of the SPRT.

1.3.2. The temperature range in. which the SPRT will be employed.

1.3.3. Specific calibration desired. (See under section 4 the list of test services that are available.)

1.3.4. Specify the type and quantity of thermometer calibration tables desired. (See under section 4.3 the list of tables that are available.)

1.3.5. If more than one type of calibration service, e.g., the calibration of standard resistors is also being purchased from NBS on the same purchase order, enough copies of purchase orders should be prepared so that one copy can accompany each type of instrument to be tested.

1.3.6. If insurance of the return shipment is desired it must be requested. Such insurance is recommended.

\section{Shipment of SPRT to NBS for the Calibration Service}

2.1. Direct shipments of SPRT by common carrier to the attention of:

Temperature Section, Heat Division

B04 Physics Building

National Bureau of Standards

Route $70 \mathrm{~S}$ and Quince Orchard Road

Gaithersburg, Maryland 20760

2.2. Precautions should be exercised to protect the SPRT from mechanical shock that would alter its calibration. In shipment, the SPRT must be softly supported within a rigid case but must not be free to "rattle" within the case. The packing material must, therefore, be resilient and not become compacted. An outer case must be sufficiently rigid and strong to withstand the treatment usually given by shippers. Styrofoam is not sufficiently rigid to be used as an outer case. The SPRT's will not be returned in containers that are obviously unsuitable, such as those closed by nailing; a wooden box should be closed with screws. Suitable containers will be provided, for a fee, whenever the SPRT shipping container is not satisfactory for re-use. The user shall assume the responsibility of instructing his shipping department concerning packaging and the desired carrier for shipping his SPRT.

Two methods suitable for packaging the SPRT for shipment are illustrated in figures 4 and 5 of section 4.7. 


\section{Requirements to Qualify for Regular Calibration Schedule}

\subsection{Quality:}

The SPRT must be a four-terminal resistor that conforms with the IPTS-68 specifications for construction practice and quality (see Appendix A).

\subsection{Dimensions:}

\subsubsection{Long stem type SPRT}

3.2.1.1. The maximum length of the resistance element shall be $5 \mathrm{~cm}$. The entire element must be located near one end of the SPRT.

3.2.1.2. The maximum outside dimension of the SPRT sheath shall be $7.52 \mathrm{~mm}\left(0.296^{\prime \prime}\right)$ for a minimum length of $46 \mathrm{~cm}\left(18^{\prime \prime}\right)$.

3.2.1.3. The maximum overall length of glass-sheathed SPRT's shall be 1 meter. The maximum overall length of SPRT's that have sheaths of thin metal is limited by the height to the ceiling of the NBS calibration laboratory: SPRT's up to 4 meters can be accommodated.

3.2.1.4. The suitability of the SPRT for calibration at NBS also depends upon the immersion characteristics of the thermometer. Although the thermometer may meet the preceding requirements of dimension, the immersion characteristics of the thermometer may be too poor for calibrations that employ the existing equipment at NBS.

\subsubsection{Capsule type SPRT}

3.2.2.1. The maximum length of the resistance element shall be $5 \mathrm{~cm}$. The element must be located at one end of the SPRT.

3.2.2.2. The maximum over-all length of the SPRT shall be $8 \mathrm{~cm}$.

3.2.2.3. The sheath, at the end that contains the resistant element, shall be reasonably uniform and shall not exceed $5.72 \mathrm{~mm}\left(0.225^{\prime \prime}\right)$ outside diameter for a minimum length of $5 \mathrm{~cm}$ and maximum length of $6 \mathrm{~cm}$.

3.2.2.4. The outside diameter of any part of the SPRT, including the head where the four leads emerge through a hermetic seal, shall not exceed $9.6 \mathrm{~mm}\left(0.38^{\prime \prime}\right)$; the length of the enlarged section shall not exceed $2 \mathrm{~cm}$.

\section{Calibration Services}

\subsection{Long stem SPRT}

4.1.1. Calibration at the triple point of water, tin point, and zinc point. A table will be furnished with entries at 1-degree intervals between -50 and $+500^{\circ} \mathrm{C}$.

4.1.2. Calibration at the triple point of water, tin point, zinc point, and oxygen normal boiling point. A table will be furnished with entries at 1-degree intervals between -183 and $+500^{\circ} \mathrm{C}$.

4.1.3. Thermometers which will withstand temperatures above $500{ }^{\circ} \mathrm{C}$, such as quartz sheathed thermometers, calibrated under 4.1 .1 or 4.1 .2 will be furnished tables to $631^{\circ} \mathrm{C}$.

\subsection{Capsule type SPRT}

The capsule type SPRT's are not generally calibrated above the tin-point temperature at the NBS. Because of the uncertain electrical insulation characteristics between the leads at the zinc point, any calibration performed with the capsule type SPRT's at the zinc point will not be of as high a quality as is obtained with the long stem SPRT's. Instead, the thermometer constant $B$ is assumed on the basis of the values of B for other SPRT's calibrated at NBS during the past few years.

4.2.1. Calibration at the triple point of water and tin point. A table will be furnished with entries at 1 -degree intervals between -50 and $+250^{\circ} \mathrm{C}$.

4.2.2. Calibration at the triple point of water, tin point, and oxygen normal boiling point. A table will be furnished with entries at 1-degree intervals between -183 and $+250^{\circ} \mathrm{C}$.

4.2.3. Calibration at the triple point of water, the tin point and a comparison calibration between 13 and $90 \mathrm{~K}$. A table will be furnished with entries at 0.1 -degree intervals between 13 and $90 \mathrm{~K}$ and at 1-degree intervals between 90 and $600 \mathrm{~K}$.

\subsection{Tables}

The user has the choice, as a part of the calibration service, as to the form of table for his thermometer.

4.3.1. The argument (the temperature) can be ${ }^{\circ} \mathrm{C},{ }^{\circ} \mathrm{F}$, or $\mathrm{K}$.

4.3.2. The function can be the resistance ratio $R(t) / R(0)$, the resistance $R(t)$, or the resistance difference $R(t)-R(0)$. For most applications the resistance ration $R(t) / R(0)$ versus temperature is recommended.

4.3.3. If no specific: request is made, the table furnished for the long stem SPRT will be the resist ance ratio $R(t) / R(0)$ versus temperature in ${ }^{\circ} \mathrm{C}$. The table furnished for the capsule type SPRT will be the resistance versus temperature in $\mathrm{K}$ whenever the comparison calibration between 13 and $90 \mathrm{~K}$ is requested; otherwise the table furnished will be the resistance ratio $R(t) / R(0)$ versus temperature in ${ }^{\circ} \mathrm{C}$.

\section{Non-Standard Platinum Resistance Thermometers}

A very special configuration of platinum resistance thermometer may be desired for certain applications. For the calibration of such thermometers the user may contact the NBS (see 1.1.) concerning the possibility of a calibration at NBS or advice on the calibration of the thermometer in the user's laboratory employing a SPRT that has been calibrated at the NBS. The information on dimensions and wiring configuration should be furnished with the inquiry. If the calibration can be performed at NBS, the fee for the special calibration will be furnished.

Calorimetric type platinum resistance thermometers, which have the resistance element wound on a flat mica sheet and sandwiched between two 
mica sheets and then inserted into a flattened tube, do not meet the specifications of the IPTS-68. A calibration of these thermometers may be furnished by NBS through special arrangement.

Inquiries concerning the calibration of other resistance thermometers, such as germanium resistance thermometers, are welcomed. The NBS will attempt, whenever possible, to meet reasonable calibration requests of services; the cost of such services will be negotiated. Appropriate discussion should take place directly between the user and the NBS technical staff. 
FORM NBS-114A (1-71)

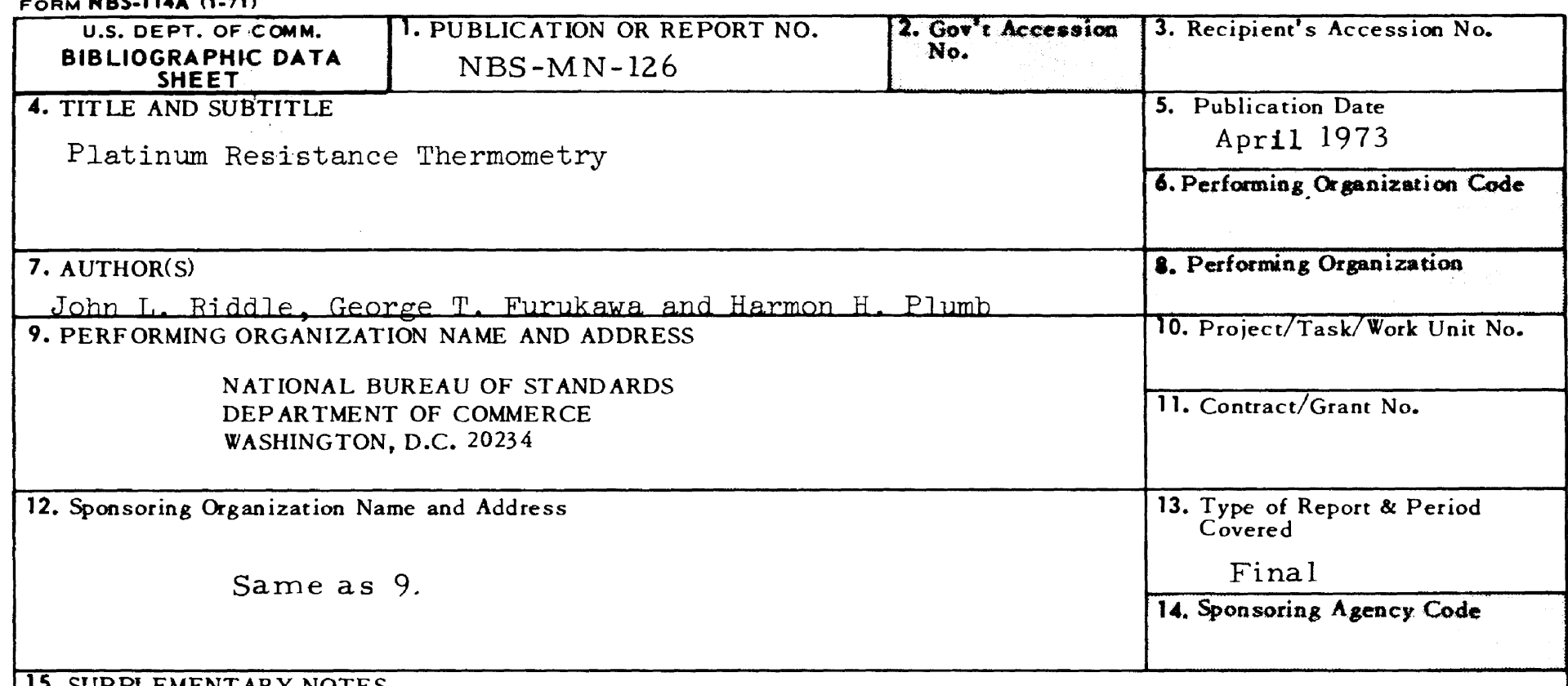

15. SUP PLEMENT ARY NOTES

16. ABSTRACT ( $A$ 200-word or less factual summary of most significant information. If document includes a significant bibliography or literature survey, mention it here.)

The monograph describes the methods and equipment employed at the National Bureau of Standards for calibrating standard platinum resistance thermometers (SPRT) on the International Practical Temperature Scale (IPTS-68). The official text of the scale is clarified and characteristics of the scale are described. Several designs of SPRT's are shown and discussed in the light of the requirements and recommendations on the text of the IPIS-68. Possible sources of error such as those due to the internal and external self-heating effects and the immersion characteristics of SPRT's are described in detail. Precautions and limitations for the mechanical and thermal treatment and for the shipment of SPRT's are indicated, and a guide is given for those desiring the thermometer calibration services of NBS. The description of equipment employed at the National Bureau of Standards for maintaining the IPTS-68 includes the triple point of water cell, tin point cell, zinc point cell, oxygen normal boiling point comparison cryostat, the 13 to $90 \mathrm{~K}$ comparison cryostat, and the reference SPRT's upon which the NBS-IPTS-68 in the region 13 to $90 \mathrm{~K}$ is based. Methods are given for calculating temperatures from the calibration data and observed resistances; the propagation of calibration errors is discussed. Supplemental information given in the Appendices includes the authorized English version of the text of the IPTS-68, tabular values of the "reference function" used below $0{ }^{\circ} \mathrm{C}$, tublar values of the differences between IPTS-68 and IPTS-48, analysis of the first derivatives at $0{ }^{\circ} \mathrm{C}$ of the IPTS-68 formulations, methods for calibrating potentiometers and Mueller bridges, and the derivation of the coefficients used in the analysis of error propagation.

17. KEY WORDS (Alphabetical order, separated by semicolons) Calibration; calibration errors; cryostat; fixed points; freezing point; International Practical Temperature Scale; platinum resistance thermometer; Thermodynamic Temperature Scale; thermometry; triple point.

18. AVAILABILITY STATEMENT

\section{$X$ UNLIMITED.}

FOR OFFICIAL DISTRIBUTION. DO NOT RELEASE TO NTIS.

\begin{tabular}{|l|c|}
\hline $\begin{array}{l}\text { 19. SECURITY CLASS } \\
\text { (THIS REPORT) }\end{array}$ & 129 \\
UNCL ASSIF IED & \\
\hline 20. SECURITY CLASS & 22. Price \\
(THIS P AGE) & $\$ 2.10$ \\
UNCL ASSIFIED & domestic \\
\hline
\end{tabular}




\section{NBS TECHNICAL PUBLICATIONS}

\section{PERIODICALS}

JOURNAL OF RESEARCH reports National Bureau of Standards research and development in physics, mathematics, and chemistry. Comprehensive scientific papers give complete details of the work, including laboratory data, experimental procedures, and theoretical and mathematical analyses. Illustrated with photographs, drawings, and charts. Includes listings of other NBS papers as issued.

Published in two sections, available separately:

\section{- Physies and Chemistry (Section A)}

Papers of interest primarily to scientists working in these fields. This section covers a broad range of physical and chemical research, with major emphasis on standards of physical measurement, fundamental constants, and properties of matter. Issued six times a year. Annual subscription: Domestic, \$17.00; Foreign, $\$ 21.25$.

\section{- Mathematical Sciences (Section B)}

Studies and compilations designed mainly for the mathematician and theoretical physicist. Topics in mathematical statistics, theory of experiment design, numerical analysis, theoretical physics and chemistry, logical design and programming of computers and computer systems. Short numerical tables. Issued quarterly. Annual subscription: Domestic, $\$ 9.00$; Foreign, $\$ 11.25$.

\section{TECHNICAL NEWS BULLETIN}

The best single source of information concerning the Bureau's measurement, research, developmental, cooperative, and publication activities, this monthly publication is designed for the industry-oriented individual whose daily work involves intimate contact with science and technology-for engineers, chemists, physicists, research managers, product-development managers, and company executives. Includes listing of all NBS papers as issued. Annual subscription: Domestic, \$6.50; Foreign, \$8.25.

\section{NOAPERHOOICALS}

Applied Mathematics Series. Mathematical tables, manuals, and studies.

Building Science Series. Research results, test methods, and performance criteria of building matcrials, components, systems, and structures.

Handbooks. Recommended codes of engineering and industrial practice (including safety codes) developed in cooperation with interested industries, professional organizations, and regulatory bodies.

Special Publications. Proceedings of NBS conferences, bibliographies, annual reports, wall charts, pamphlets, etc.

Monographs. Major contributions to the technical literature on various subjects related to the Bureau's scientific and technical activities.

National Standard Reference Data Series. NSRDS provides quantitative data on the physical and chemical properties of materials, compiled from the world's literature and critically evaluated.

Product Standards. Provide requirements for sizes, types, quality, and methods for testing various industrial products. These standards are developed cooperatively with interested Government and industry groups and provide the basis for common understanding of product characteristics for both buyers and sellers. Their use is voluntary.

Technical Notes. This series consists of communications and reports (covering both other-agency and NBS-sponsored work) of limited or transitory interest.

Federal Information Processing Standards Publications. This series is the official publication within the Federal Government for information on standards adopted and promulgated under the Public Law 89-306, and Bureau of the Budget Circular A-86 entitled, Standardization of Data Elements and Codes in Data Systems.

Consumer Information Series. Practical information, based on NBS research and experience, covering areas of interest to the consumer. Easily understandable language and illustrations provide useful background knowledge for shopping in today's technological marketplace.

\section{BIBLIOGRAPHIC SUBSCRIPTION SERVICES}

The following current-awareness and literature-survey bibliographies are issued periodically by the Bureau :

'yyogenic Data Center Current Awareness Service (Publications and Reports of Interest in Cryogenics). A literature survey issued weekly. Annual subscription : Domestic, $\$ 20.00$; foreign, $\$ 25.00$.

Liquefied Natural Gas. A literature survey issued quarterly. Annual subscription: $\$ 20.00$.

Superconducting Devices and Materials. A literature survey issued quarterly. Annual subscription: $\$ 20.00$. Send subscription orders and remittances for the preceding bibliographic services to the U.S. Department of Commerce, National Technical Information Service, Springfield, Va. 22151.

Electromagnetic Metrology Current Awareness Service (Abstracts of Selected Articles on Measurement Techniques and Standards of Electromagnetic Quantities from D-C to Millimeter-Wave Frequencies). Issued monthly. Annual subscription: $\$ 100.00$ (Special rates for multi-subscriptions). Send subscription order and remittance to the Electromagnetic Metrology Information Center, Electromagnetics Division, National Bureau of Standards, Boulder, Colo. 80302.

Order NBS publications (except Bibliographic Subscription Services) from: Superintendent of Documents, Government Printing Office, Washington, D.C. 20402. 
U.s. DEPARTMENT OF COMMERCE National Bureau of Standards

Washington, D.C. 20234

OFFICIAL BUSINEBS

Penalty for Private Use. $\mathbf{\$ 3 0 0}$
POSTAGE AND FEES PAID

U.S. DEPARTMENT OF COMMEACE

215

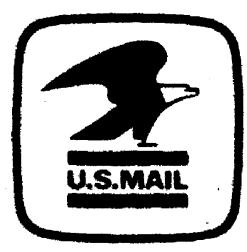

